\title{
Episodic memory, amnesia, and the hippocampal-anterior thalamic axis
}

\author{
John P. Aggleton \\ School of Psychology, Cardiff University, Cardiff, CF1 3YG, Wales \\ aggleton@cardiff.ac.uk www.cf.ac.uk/uwc/psych/ \\ Malcolm W. Brown \\ Department of Anatomy, University of Bristol, Bristol, BS8 1TD, \\ United Kingdom m.w.brown@bristol.ac.uk
}

\begin{abstract}
By utilizing new information from both clinical and experimental (lesion, electrophysiological, and gene-activation) studies with animals, the anatomy underlying anterograde amnesia has been reformulated. The distinction between temporal lobe and diencephalic amnesia is of limited value in that a common feature of anterograde amnesia is damage to part of an "extended hippocampal system" comprising the hippocampus, the fornix, the mamillary bodies, and the anterior thalamic nuclei. This view, which can be traced back to Delay and Brion (1969), differs from other recent models in placing critical importance on the efferents from the hippocampus via the fornix to the diencephalon. These are necessary for the encoding and, hence, the effective subsequent recall of episodic memory. An additional feature of this hippocampal-anterior thalamic axis is the presence of projections back from the diencephalon to the temporal cortex and hippocampus that also support episodic memory. In contrast, this hippocampal system is not required for tests of item recognition that primarily tax familiarity judgements. Familiarity judgements reflect an independent process that depends on a distinct system involving the perirhinal cortex of the temporal lobe and the medial dorsal nucleus of the thalamus. In the large majority of amnesic cases both the hippocampal-anterior thalamic and the perirhinal-medial dorsal thalamic systems are compromised, leading to severe deficits in both recall and recognition.
\end{abstract}

Keywords: amnesia; fornix; hippocampus; memory; temporal cortex; thalamus

\section{Introduction}

This target article describes how medial temporal lobemedial diencephalic interactions contribute to episodic memory. Previous models have focussed on neural circuitry within the temporal lobe. This earlier focus on "temporal lobe memory systems" arose from a number of assumptions about amnesia and models for amnesia. This target article questions these assumptions, and from this emerges a different way of considering the neural substrates of episodic memory. At the centre of this revision is the notion that the link from the hippocampus to the mamillary bodies and anterior thalamic nuclei, via the fornix, is critical for normal episodic memory (Gaffan 1992a). Moreover, damage to this axis is responsible for the core deficits in anterograde amnesia, as was originally proposed by Delay and Brion (1969). To understand why this view became unpopular and why it has now reemerged, it is necessary to describe how a number of past findings have been interpreted.

We will first describe the main features of the proposed model. This is followed by a section summarising relevant evidence from studies of amnesia and animal models of amnesia, describing the way this evidence has often been interpreted. Section 4 examines certain assumptions underlying previous interpretations and shows that existing evidence can be reinterpreted in a different way. Section 5
John AgGLETon has been Professor of Cognitive Neuroscience at Cardiff University, Wales, since 1994. Prior to that he worked at Durham University for 11 years. His research has focussed on the role of limbic brain regions in memory and emotion, both in humans and in a range of animal species. He is the author of over one hundred scientific publications, which include a paper on his favourite soap opera (The Archers) and a paper on dead cricketers! He has edited a book on the amygdala (The Amygdala, 1992, Wiley-Liss, New York) and is currently editing a follow-up book on the same brain structure.

Malcolm Brown is Professor of Cognitive Neuroscience and Anatomy and a member of the MRC Centre for Synaptic Plasticity at the University of Bristol, England. He took a first degree in theoretical physics before a Ph.D. in neuroscience. His research centres on studies of the neural basis of memory, particularly recognition memory. He is the author of over one hundred scientific publications in the area of neuroscience, including papers on hippocampal synaptic plasticity, imprinting, and benzodiazepine- and pregnancy-induced human amnesia, as well as recognition memory. 
Aggleton \& Brown: Episodic memory, amnesia, and hippocampus

describes new evidence from behavioural studies, human clinical studies, single-unit recording studies, and brain activation studies that provide further support for the proposed model of medial temporal-medial diencephalic interactions. The final sections consider some of the implications of the model. Throughout this review we have drawn on evidence from studies of animals when the clinical data lack sufficient anatomical resolution. Great care is needed when transposing results across species (Tulving \& Markowitsch 1994), and biases can be introduced by the reliance on one particular research method. For these reasons we have tried, wherever possible, to present complementary data from an array of techniques and from more than one species.

\section{Main features of the proposed model}

1. The anatomical focus of the model concerns the connections between the hippocampus, the mamillary bodies, and the medial thalamus. (As a matter of terminology the term hippocampus is used here to refer to the hippocampal fields CA1-4, the dentate gyrus, and the subicular complex. The mamillary bodies and the medial thalamus are both medial components of the diencephalon, which is composed of the thalamus, hypothalamus, epithalamus, and subthalamus). The hippocampal efferents to the medial diencephalon are regarded as vital for normal hippocampal activity and are, hence, seen as functional extensions of the hippocampus (Fig. 1). The principal thalamic targets in this system are the anterior thalamic nuclei. These nuclei receive direct hippocampal projections via the fornix, and indirect hippocampal projections via the mamillary bodies and the mamillothalamic tract. Other thalamic nuclei that may contribute to this system are the rostral midline nuclei and the lateral dorsal nucleus.

2 . The system beyond the anterior thalamic nuclei becomes more diffuse, but one component projects back from the anterior thalamic nuclei to the hippocampus and to adjacent temporal cortical regions. These return connections, which mainly use the cingulum bundle, form part of a circuit that permits these diencephalic regions to influence temporal lobe processing. Other important outputs are to the cingulate and prefrontal cortices. A consequence of the diffuseness of the system beyond the anterior thalamic nuclei is that damage in the relevant tracts or regions (e.g., cingulum bundle and prefrontal cortex) has a less profound impact upon episodic memory.

3. This extended hippocampal-diencephalic system is critical for the efficient encoding and, hence, normal recall of new episodic information. As a consequence, damage to the component structures can result in anterograde amnesia; a common feature of all diencephalic and temporal lobe amnesias is the bilateral involvement of part of this "extended hippocampal-diencephalic system" (i.e., the hippocampus, fornix, mamillary bodies, anterior thalamus, and, possibly, cingulum bundle). Furthermore, damage to different parts of this system produces similar memory impairments.

4. In contrast, this extended hippocampal-diencephalic system need not be vital for efficient recognition. This is because recognition is regarded as being composed of at least two independent processes (Mandler 1980), only one of which is hippocampally dependent. Thus item recognition

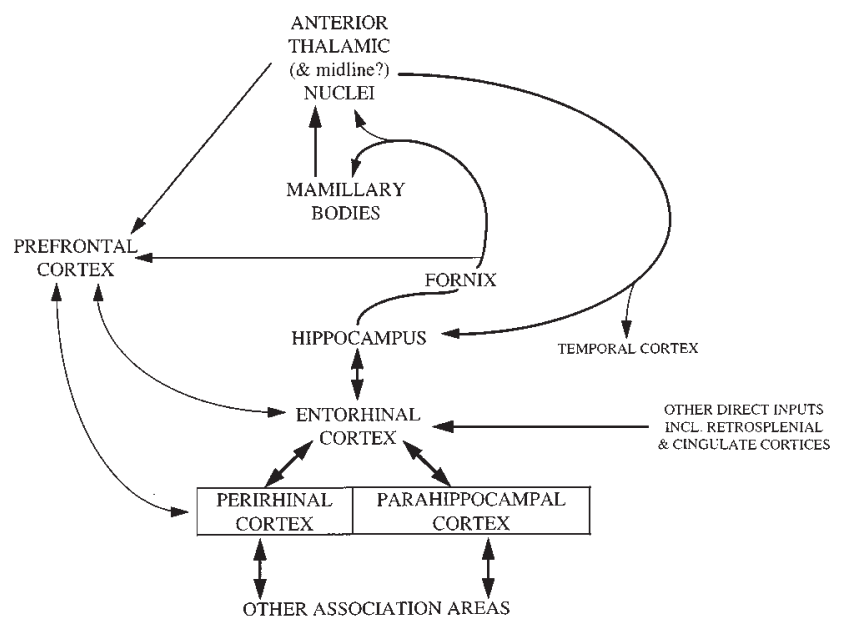

Figure 1. Schematic diagram of the principal pathways that allow the encoding of episodic information and underlie recollective aspects of recognition. The relative thickness of the lines indicates the putative importance of the various connections.

occurs through recollection of the stimulus ("remembering"), a process that is hippocampally dependent, and by detecting stimulus familiarity ("knowing"), which does not require the hippocampus. The latter process is especially dependent on the perirhinal cortex in the temporal lobes.

5. Although the hippocampus and perirhinal cortex are anatomically linked, they are not necessarily dependent on each other for their respective roles in the encoding of episodic information and familiarity-based recognition. In particular, both the hippocampus and perirhinal cortex have independent links with other association cortical areas.

6. Although the hippocampus is closely linked to the anterior thalamic nuclei, the perirhinal cortex is connected with the medial dorsal thalamic nucleus. These two parallel temporal-thalamic systems (Fig. 2) make qualitatively different contributions to learning and memory. The entorhinal cortex has attributes of both systems.

7. The traditional distinction between temporal lobe and diencephalic amnesics is misleading; both groups have damage to the same functional system. Nevertheless, the large majority of amnesics have additional pathology in certain subcortical and cortical sites, and this can extend the nature of the memory loss so that it involves other aspects of memory.

8. The proposed hippocampal-diencephalic system is required for the encoding of episodic information, permitting the information to be set in its spatial and temporal context ("episode"), so aiding subsequent retrieval and reducing interference (i.e., heightening discriminability).

9 . The prefrontal cortex interacts with both of these systems at a variety of levels, engaging efficient encoding strategies that can then aid subsequent recall.

\section{Studies of amnesia and animal models of amnesia, and their interpretation}

\subsection{Neuropathological evidence}

Anterograde amnesia is typified by a failure to acquire or retain "episodic" information (Tulving 1983) that occurred af- 
Aggleton \& Brown: Episodic memory, amnesia, and hippocampus

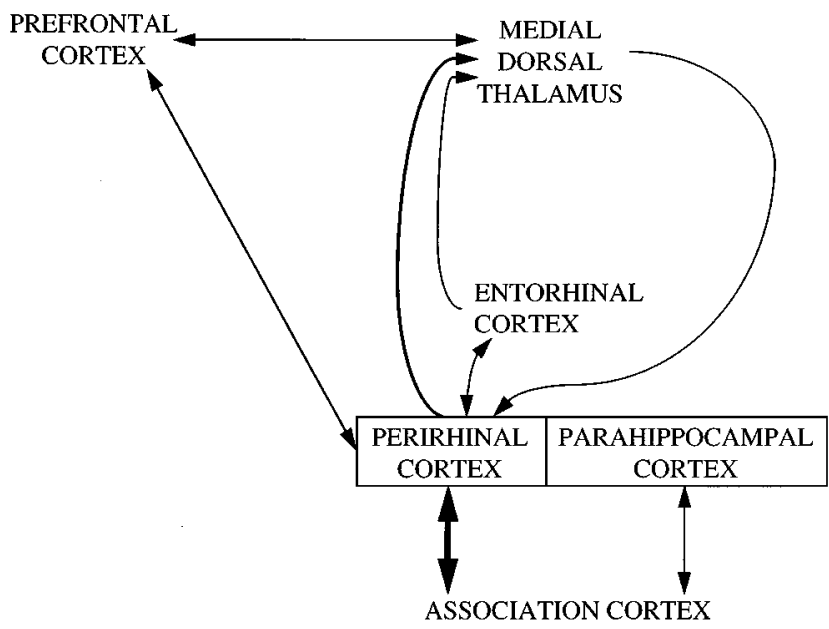

Figure 2. Schematic diagram of the principal pathways underlying the detection of item familiarity. The relative thickness of the lines indicates the putative importance of the various connections.

ter the onset of brain injury. Damage in more than one brain region can result in anterograde amnesia, and neuropathological studies have repeatedly highlighted the medial temporal lobes and the medial diencephalon. Identifying the critical structures has, however, proved to be surprisingly difficult. Although it is often assumed that temporal lobe amnesia is principally a consequence of damage to the hippocampus, it remains to be confirmed whether such damage is sufficient to induce amnesia. Relevant evidence has come from amnesic cases with discrete unilateral hippocampal damage in one hemisphere combined with more extensive temporal lobe damage in the other hemisphere (Penfield \& Mathieson 1974; Woods et al. 1982). If bilateral damage is required to induce amnesia, such cases strongly implicate the hippocampus. Other evidence has come from amnesics with confirmed bilateral pathology restricted to the hippocampus and the adjacent parahippocampal gyrus or uncus (DeJong et al. 1969; Glees \& Griffiths 1952). Some of the most convincing evidence has, however, come from the discovery that hypoxia can produce both a permanent anterograde amnesia and discrete bilateral hippocampal pathology (Cummings et al. 1984; Rempel-Clower et al. 1996; Victor \& Agamonolis 1990; Zola-Morgan et al. 1986). There is, however, debate over whether these patients suffer "hidden" pathology (see sect. 4.1), so there is still a need to confirm whether discrete, bilateral hippocampal damage can induce anterograde amnesia.

Diencephalic amnesia appears to be even more complex; neuropathological evidence has implicated several structures, namely the mamillary bodies, the anterior thalamic nuclei, the medial dorsal thalamic nucleus, and the parataenial thalamic nucleus (Aggleton \& Sahgal 1993; Clarke et al. 1994; Dusoir et al. 1990; Mair et al. 1979; Markowitsch 1982; Parkin \& Leng 1993). A number of adjacent tracts (the mamillothalamic tract and the internal medullary lamina) have also been implicated (Markowitsch 1988; Savage et al. 1997). Unfortunately, there are still no amnesic cases with confirmed, circumscribed damage in just one of these structures. Furthermore, the proximity of these nuclei to one another, along with the likelihood of damage to fibres of passage and adjacent tracts, makes it extremely unlikely that unambiguous cases will be discovered.

\subsection{Testing recognition to assess anterograde amnesia in animals}

The lack of unambiguous clinical evidence has led researchers to model anterograde amnesia in animals, and thus test unusually selective lesions. A prerequisite, however, is the development of behavioural tasks that tax the same classes of memory that are lost in amnesia. This need is underscored by the many examples of spared learning abilities in amnesia, which include classical conditioning, visuomotor skill tasks, and priming (Parkin \& Leng 1993; Schacter et al. 1993; Weiskrantz 1990).

Studies with animals have, in fact, relied very heavily on behavioural tests of recognition. This is because a loss of recognition is a striking feature of anterograde amnesia and has been regarded as a core deficit (Haist et al. 1992; Parkin \& Leng 1993; Squire \& Knowlton, 1995; Squire \& Shimamura 1986). Furthermore, the use of forced-choice designs makes it relatively easy to test animals. In contrast, examining the recall of episodic information by animals has proved much more problematic. As a consequence the favoured test of recognition, delayed nonmatching-to-sample (DNMS), has become the litmus test for models of anterograde amnesia.

In DNMS the animal is first shown a sample stimulus (often a "junk" object). After a delay the animal is shown that same object along with a novel or less familiar object. Selection of the novel object (nonmatching) is rewarded in DNMS, whereas in delayed matching-to-sample (DMS) selection of the familiar object is rewarded. In the "trialunique" version of DNMS and DMS both the novel and the familiar objects are then discarded so that new items can be used for the next sample and the next novel alternative. Early studies using the trial-unique version of the DNMS task with monkeys soon confirmed that, as in people, large medial temporal lesions (Mishkin 1978; ZolaMorgan et al. 1982) and large medial diencephalic lesions (Aggleton \& Mishkin 1983a; 1983b) produce very severe recognition deficits. The apparent validity of these recognition tests was further strengthened by studies showing that people with either temporal lobe or diencephalic amnesia are markedly impaired on forced-choice recognition tasks designed to be analogous to the DNMS and DMS tasks given to monkeys (Aggleton et al. 1988; Squire et al. 1988). It is therefore not surprising that these tasks have been used to assess the effects of selective bilateral damage in a number of key sites.

\subsection{Testing the contribution of the fornix}

One site of special interest has been the fornix. Among its components this tract contains the cholinergic innervation to the hippocampus from the medial septum, as well as hippocampal efferents to the diencephalon, striatum, and prefrontal cortex. These efferents include dense projections to the mamillary bodies and the anterior thalamic nuclei, which in monkeys are conveyed solely in the fornix (Aggleton et al. 1986a; Aggleton \& Saunders 1997). As a consequence, the fornix forms a vital bridge between medial temporal and medial diencephalic regions implicated in anterograde amnesia.

Although the first study to use the DMS task to assess the effects of fornix transection reported an impairment (Gaffan 1974), a series of later DNMS and DMS studies found that fornix transection produced little or no recog- 
nition deficit in monkeys (Bachevalier et al. 1985a; 1985b; Gaffan et al. 1984; Zola-Morgan et al. 1989a) and spared DNMS performance in rats (Aggleton et al. 1990; Rothblat \& Kromer 1991; Shaw \& Aggleton 1993). Similarly, fornix lesions were found to have no effect on spontaneous tests of object recognition (Ennaceur \& Aggleton 1994; Ennaceur et al. 1996; 1997). Indeed, in one study, monkeys with fornix lesions eventually performed the DNMS task significantly better than control animals (Zola-Morgan et al. 1989a); in another study, monkeys with fornix lesions showed enhanced preference for perceptual novelty (Zola-Morgan et al. 1983). Similarly, rats with fornix lesions were able to acquire a DNMS task more rapidly than control animals (Shaw \& Aggleton 1993). The immediate conclusion was that fornix damage did not disrupt recognition and, hence, was not sufficient to induce anterograde amnesia (Squire \& Zola-Morgan 1991; Zola-Morgan et al. 1989a).

This conclusion was consistent with a review of memory loss and fornix damage in humans (Garcia-Bengochea \& Friedman 1987). Among 142 patients thought to have bilateral fornicotomy for the treatment of epilepsy, none had persistent memory problems. A further 13 cases with fornix damage associated with third ventricle colloid cysts were also considered. Four of them had persistent memory loss (Carmel 1985; Garcia-Bengochea \& Friedman 1987; Sweet et al. 1959), but the likelihood that the cysts had caused additional diencephalic damage weakened the value of these individual cases. Similar constraints can be applied to other patients in whom surgery for cysts or tumours resulted in both fornix damage and memory loss (Cameron \& Archibold 1981; Geffen et al. 1980; Heilman \& Sypert 1977; Tucker et al. 1988). Additional problems of interpretation arise with those patients in whom the hippocampal commissures as well as the fornix were cut or disconnected (Heilman \& Sypert 1977; Tucker et al. 1988). Although Hassler (1962) described a woman in whom stereotaxic coagulation of the fornices led to an amnesic state, the woman survived only a few days after surgery, severely limiting assessment. Taken together, the cases with presumed fornix damage and apparently unchanged memory (Garcia-Bengochea \& Friedman 1987; see also Woolsey \& Nelson 1975) far outnumbered the few single case studies in which fornix damage appeared to be associated with amnesia.

Other evidence has come from studies on the mamillary bodies, which the fornix innervates. It had long been appreciated that mamillary body degeneration is a consistent feature of Korsakoff's disease and that it might contribute to the anterograde amnesia. A comprehensive neuropathological study by Victor and his co-workers (1971) concluded, however, that thalamic damage (and in particular damage to the medial dorsal thalamic nucleus) was a better predictor of the memory loss. Consistent with this was the finding that mamillary body lesions in animals did not disrupt DNMS performance (Aggleton \& Mishkin 1985; Aggleton et al. 1990; Zola-Morgan et al. 1989a), whereas lesions in the medial dorsal thalamic region impaired both the acquisition and performance of the DMS and DNMS tasks (Aggleton \& Mishkin 1983b; Hunt \& Aggleton, 1991; Mumby et al. 1993; Parker et al. 1997; Zola-Morgan \& Squire 1985a). Insofar as these findings failed to support a role for the mamillary bodies in anterograde amnesia they accorded with similar evidence for the fornix.

\subsection{Comparing the effects of lesions in the hippocampus and lesions in adjacent cortical regions}

Studies with animals also provided the opportunity to examine the effects of increasingly selective lesions within the temporal lobe. Aspiration lesions of the hippocampus consistently produced a modest, but significant, DNMS deficit (Murray \& Mishkin 1986; Zola-Morgan \& Squire 1986; Zola-Morgan et al. 1989a; 1993), supporting the contribution of this structure to amnesia. More discrete temporal lobe lesions also revealed that the amygdala was not critical (O’Boyle et al. 1993; Zola-Morgan et al. 1989b). Much more surprising was the discovery that the cortex immediately lateral to the amygdala and hippocampus is of vital importance for DNMS performance. Thus lesions involving the rhinal region (comprising the perirhinal and entorhinal cortices) or more extensive lesions involving the rhinal region and the parahippocampal gyrus produce extremely severe and persistent DNMS deficits (Meunier et al. 1993; Mumby \& Pinel 1994; Murray 1996; Murray \& Mishkin 1986; Suzuki et al. 1993; Zola-Morgan et al. 1989b). More discrete lesions within the rhinal region have since highlighted the special importance of the perirhinal cortex (Meunier et al. 1993; 1996). In contrast, entorhinal lesions produce only a very mild or transient impairment (Leonard et al. 1995; Meunier et al. 1993). Similarly, removal of parahippocampal cortex does not contribute to the DNMS deficit (Meunier et al. 1996; Ramus et al. 1994). These findings, along with those from single unit recording studies (see sect. 5.3), have forced a fundamental reappraisal of the contribution of individual temporal lobe structures to memory (Murray 1996).

Anatomical studies have shown that the perirhinal and parahippocampal cortices project densely upon the entorhinal cortex, and, in fact, they provide nearly two thirds of the cortical inputs to the entorhinal cortex (Insausti et al. 1987; Suzuki \& Amaral 1994). The entorhinal cortex itself is the major source of afferents to the hippocampus. As a consequence these indirect connections, along with a number of direct perirhinal-hippocampal projections (Suzuki \& Amaral 1990; Witter \& Amaral 1991), ensure that the perirhinal and parahippocampal cortical areas are a major source of hippocampal inputs. Additionally, the hippocampus has extensive reciprocal connections with the entorhinal, perirhinal, and parahippocampal cortices (Suzuki 1996a; Suzuki \& Amaral 1994; Witter et al. 1989). These interconnections help to reinforce the view that the hippocampus along with the perirhinal, parahippocampal, and entorhinal cortices function as a closely integrated unit subserving aspects of memory, including recognition (Squire \& Zola-Morgan 1991). It should be emphasized, however, that the DNMS deficit following perirhinal removal is not due simply to a disconnection of hippocampal inputs; the severity of this deficit is considerably greater than that found after hippocampectomy (Meunier et al. 1996; Murray 1996; Zola-Morgan et al. 1993). Thus the perirhinal region must have independent mnemonic capabilities.

\subsection{The temporal lobes and episodic memory: Current models}

These new findings have been integrated with growing clinical evidence suggesting that hippocampal damage is suffi- 
cient to induce amnesia, and they have led to a number of influential models of temporal lobe function. A common feature of these models is that the perirhinal, entorhinal, and parahippocampal cortices, along with the hippocampus, form the key components of a closely integrated temporal lobe memory system. This system is reciprocal; the plentiful projections back from the hippocampus to the entorhinal cortex and the perirhinal/parahippocampal cortices are seen as instrumental in setting up long-term representations (i.e., memories) in neocortex (Eichenbaum et al. 1994; Squire \& Knowlton 1995; Squire \& Zola-Morgan 1991; Suzuki 1996a; 1996b). One important consequence of the reciprocal nature of these interactions is that the proposed systems are largely self-contained within the temporal lobes. This has served to distance other structures such as the fornix, anterior thalamic nuclei, and mamillary bodies and implies that the involvement of these regions in diencephalic amnesia will reflect a qualitatively different syndrome.

In one of the most often cited models (Squire \& ZolaMorgan 1991) the parahippocampal, perirhinal, and entorhinal cortices form a reciprocal network with the hippocampus to create a "medial temporal memory system." This system is crucial for the rapid acquisition of new information about facts and events, which then gradually becomes consolidated in the neocortex and eventually becomes independent of the hippocampus (Squire \& Alvarez 1995; Squire \& Zola-Morgan 1991). The role of the hippocampus is to bind together different components of the memory. Later expansions of this model have acknowledged some linkage with medial thalamic regions, but no apparent role has been provided for hippocampal outputs to the mamillary bodies and anterior thalamus via the fornix (Squire \& Knowlton 1995; Zola-Morgan \& Squire 1993). This exclusion stems from the failure of either fornix or mamillary body lesions to disrupt DNMS performance, and the assumption that there is a close relationship between recognition and recall (Haist et al. 1992; Squire \& Knowlton 1995). It is therefore presumed that these connections are not necessary for the recall of episodic (declarative) memory.

A related model (Eichenbaum et al. 1994) proposes a "hippocampal memory system" formed by the hippocampus and the "parahippocampal region" (comprising the entorhinal, perirhinal, and parahippocampal cortices). This hippocampal memory system contributes both to the temporary maintenance of memories and to the processing of a particular type of memory representation. In particular, the parahippocampal region supports intermediate-term storage of individual items, whereas the hippocampal formation is concerned with organizing memories according to relevant relationships between items, including spatial relationships (Eichenbaum et al. 1994). This "hippocampal memory system" is seen to be critical for episodic memory, so dysfunction of the system can lead to anterograde amnesia.

\section{A critical examination of key assumptions underlying these models of the neural substrates of recognition and recall}

In developing these models of temporal lobe involvement in episodic memory, a number of different assumptions have proved very influential. These are: (1) that hippocam- pal damage is sufficient to impair recognition; (2) that testing recognition (i.e., using DNMS or DMS) provides a valid assay for the core deficits in anterograde amnesia; and (3) that hippocampal function is critically dependent on afferents from the perirhinal region. There are now, however, good grounds for questioning all these assumptions, and in doing so a quite different view of temporal lobe-diencephalic interactions emerges.

\subsection{Is hippocampal damage sufficient to impair recognition?}

The importance of the perirhinal cortex highlights the need to reexamine the effects upon DNMS of hippocampectomy using techniques that spare rhinal regions. Interestingly, lesions of the rat hippocampus are possible via a dorsal route that avoids the rhinal cortices. Hippocampectomies performed in this manner have little or no effect on DNMS tests (Aggleton et al. 1986b; Duva et al. 1997; Mumby et al. 1996; Steele \& Rawlins 1993). Another approach has been to induce ischaemic lesions, which can produce seemingly selective pathology in the hippocampus. Such lesions are accompanied by persistent DNMS deficits in both monkeys (Bachevalier \& Mishkin 1989; Zola-Morgan et al. 1992) and rats (Wood \& Phillips 1991; Wood et al. 1993). A problem is that the neural dysfunction caused by the ischaemia may be much more extensive than the region of gross pathology (Bachevalier \& Meunier 1996; Gaffan \& Lim 1991; Nunn \& Hodges 1994). Occlusion of the posterior cerebral artery, for example, results in a DNMS deficit greater than that expected from the grossly apparent brain damage (Bachevalier \& Mishkin 1989). Similarly, discrete ischaemic hippocampal lesions in rats produce marked DNMS deficits (Wood \& Phillips 1991; Wood et al. 1993), yet neurotoxic lesions intended to match the extent of the apparent ischaemic damage have no effect on DNMS performance (Duva et al. 1997). Extensive conventional hippocampal lesions not only spare DNMS performance (Mumby et al. 1996; Wood et al. 1993) but, remarkably, can attenuate the effects of ischaemia (Mumby et al. 1996). This result not only highlights the mismatch between the observed pathology and the functional pathology, but also indicates that the ischaemia resulted in extrahippocampal dysfunctions subsequent to the initial hippocampal pathology (Mumby et al. 1996). Finally, a recent positron emission tomography (PET) study (Markowitsch et al. 1997) has highlighted the limitation of relying on magnetic resonance imaging (MRI) to uncover functional damage in cases of anoxia. This is so because PET revealed widespread regions of hypoactivity in an amnesic patient that could not be predicted from MRI scans (Markowitsch et al. 1997).

The possibility that ischaemia can lead to more extensive dysfunction than that apparent by standard pathological measures has, however, been disputed (Squire \& Zola 1996). It has been argued that the DNMS deficits following posterior artery occlusion (Bachevalier \& Mishkin 1989) were exagerated by reference to unusually high scoring controls, and that monkeys with hippocampal lesions produced by sterotaxy (Alvarez et al. 1995) perform at a level comparable to those with ischaemic lesions (Squire \& Zola 1996). The first of these points requires additional control data to resolve. The second criticism is, however, potentially misleading insofar as the comparison included data from other tests, that is, those not testing recognition. When 
the data are taken only from comparable DNMS tests (delays $15 \mathrm{sec}$ to $10 \mathrm{~min}$ ), it is found that three of the four ischaemic monkeys performed at more than 2.7 standard deviations below the mean score of the stereotaxic hippocampectomy cases (Squire \& Zola 1996), whereas the control animals for the two studies performed at equivalent levels. The DNMS scores of the ischaemic animals were, however, comparable to those of monkeys with hippocampal lesions made by techniques that also damage adjacent perirhinal cortex (Bachevalier \& Meunier 1996). Although the balance of evidence indicates that anoxia can produce more extensive recognition dysfunction than that predicted from an assessment with standard histological methods, it is also clear that this key issue requires further examination (Nunn \& Hodges 1994).

For these reasons it is preferable to focus on studies that have examined selective, stereotaxic lesions within the hippocampus. In one of the few such studies, radio frequency lesions were placed bilaterally within the hippocampus (Alvarez et al. 1995). The lesions did not disrupt DNMS performance significantly until there was a delay of $10 \mathrm{~min}$ between sample presentation and test (Alvarez et al. 1995). In a number of other stereotaxic studies a neurotoxin (ibotenic acid) was injected into the monkey hippocampus, sparing fibres of passage and adjacent fibre tracts (Beason-Held et al. 1993; Murray 1996; Murray \& Mishkin 1996; O’Boyle et al. 1993). Although the first of these studies reported DNMS deficits (Beason-Held et al. 1993), the remaining studies showed normal levels of performance even though the hippocampal fields CA1-4, along with the amygdala, were destroyed. In one of these studies the retention interval was extended to $40 \mathrm{~min}$, but, unlike the case in an earlier study that had found an impairment with such delays (Alvarez et al. 1995), the animals were not removed from the apparatus during testing (Murray \& Mishkin 1996). These animals showed no DNMS impairment (Murray \& Mishkin 1996). It therefore appears that selective hippocampal lesions can often spare DNMS performance, although for some of these reports the histology remains to be published in a comprehensive form. It is also still necessary to examine the performance of monkeys with neurotoxic hippocampal lesions that include the subiculum.

The effects of these selective hippocampal lesions now closely correspond to the effects of fornix lesions on DNMS; that is, they typically have little or no effect. This is noteworthy insofar as fornix transection often mimics hippocampal dysfunction, most obviously for tests of spatial memory (Aggleton et al. 1986b; 1992; 1995a; Barnes 1988; Olton et al. 1982; Saunders \& Weiskrantz 1989). It had appeared that DNMS presented an important exception to this general rule, but these recent stereotaxic studies show that the effects of hippocampectomy and fornicotomy are in accordance for DNMS as well.

It has been argued that the lack of a clear hippocampal lesion deficit in DNMS tasks might be due to the training prior to surgery, which can then mask any subsequent lesion deficit (Alvarez et al. 1995). Because learning the nonmatching rule per se cannot help the animal solve any individual problem, it is difficult to see how greater training could obscure a deficit unless there are ceiling effects. Nevertheless, this claim has led to a number of studies of spontaneous recognition based upon preferential viewing of novel visual stimuli. With such tasks it has been reported that lesions not only of the perirhinal cortex (Clark et al.
1997) but also of the hippocampus (Clark et al. 1996) can disrupt performance at delays as short as $10 \mathrm{sec}$. Such tasks often use complex visual stimuli, and previous lesion studies have demonstrated that hippocampal system lesions impair the ability to use "scenes" that are composed of an array of different features (Gaffan 1994b). Thus the abnormal behaviour following hippocampal lesions may reflect a failure to associate the component elements. It is also the case that spontaneous tests of recognition are more prone to disruption by other factors such as hyperactivity or increased distractability. In an ingenious variant on such tasks, Honey et al. (1998) showed that neurotoxic lesions of the rat hippocampus do not affect orientation and subsequent habituation to novel visual and auditory stimuli. It was, however, found that animals with these lesions failed to orient when familiar combinations of these cross-modal stimuli were rearranged (mismatched). Thus the hippocampal lesions spared novelty detection per se, but the mismatch condition revealed a failure to detect or respond to changes in the learned association between the pairs of cross-modal stimuli (Honey et al. 1998).

The evidence showing that extensive, but selective, hippocampal damage can often spare DNMS raises the question of whether there is comparable, clinical evidence. One source of potential evidence comes from amnesic people with hypoxic damage, who are very likely to suffer hippocampal damage (but may also suffer "hidden pathology"; see above). Such amnesics can show apparently normal recognition performance in spite of impaired recall (Volpe et al. 1986). Consistent with this are the findings from a recent survey of amnesics (Aggleton \& Shaw 1996), which analysed results from a standard test of recognition, the Warrington Recognition Memory Test (RMT). The RMT (Warrington 1984) consists of two subtests, one testing face recognition, the other testing word recognition. From a sample of 112 amnesics placed in 11 distinct pathological groupings, it was found that three groups of amnesics did not differ from their age-matched norms (Aggleton \& Shaw 1996). One of these groups comprised patients with restricted hippocampal damage following hypoxia, another contained patients with fornix damage (Aggleton \& Shaw 1996; see also McMackin et al. 1995), and a third group had selective diencephalic damage. These groups not only failed to differ from the normal subjects, they also performed significantly better than some of the other amnesic groups.

Although these RMT results closely match the findings for DNMS performance by nonhuman primates (i.e., little or no effect following hippocampal or fornix damage), there are a number of important constraints. The first is that the RMT data come from just one test of recognition and, as was indicated in section 2, it is to be predicted that hippocampal damage will have more impact on some tests of recognition than on others. The second is that cases with anoxic damage may have variable covert pathology. Both of these considerations apply to a recent review of recognition following anoxic hippocampal damage in humans (Reed \& Squire 1997), which convincingly shows that this aetiology can lead to recognition deficits across a wide range of tests. Even so, compared to test norms, performance on the standard version of the RMT is apparently preserved in some of these patients and deficient in others (Reed \& Squire 1997). A related case concerns an amnesic who performed very poorly on the RMT, even though MRI studies indicated that 
the subject had circumscribed lesions confined to areas CA1 and CA2 (Kartsounis et al. 1995). This same person did, however, show very severe retrograde amnesia suggestive of more extensive cortical damage (Kapur et al. 1992; ZolaMorgan et al. 1986). In view of the fact that the amnesia arose from repeated ischaemic episodes, this apparent discrepancy might be related to the issue of hidden pathology (Bachevalier \& Mishkin 1989; Mumby et al. 1996).

Other relevant evidence comes from a recent study of 104 epileptic patients who had been tested on the RMT and had unilateral temporal lobe pathology confirmed by MRI (Baxendale 1997). Patients with combined cortical and hippocampal damage performed significantly worse than those with selective hippocampal damage. Furthermore, the group mean score of those with selective left hippocampal damage on the test of word recognition (the subtest on which they should be most impaired) was in the normal range, as was the group mean score for those with right hippocampal damage on the face recognition test (Baxendale 1997). The conclusion, that unilateral hippocampal damage had no consistent effect on this test of recognition, reinforced a previous study showing that hippocampal sclerosis had no apparent effect on either of the RMT subtests (tested preoperatively), although deficits on delayed recall were found (Miller et al. 1993). These conclusions appear to contrast with those of a recent study using event-related potentials, which showed a loss of reactivity to novel stimuli in five subjects with combined unilateral pathology in the hippocampus (Knight 1996). In all five cases, however, the pathology involved the parahippocampal gyrus and the entorhinal cortex (Knight 1996), so the resulting deficit could be predicted.

Even if it is accepted that hippocampectomy can induce a subtle DNMS deficit (Alvarez et al. 1995; but see Murray \& Mishkin 1996), this is apparent only after very lengthy delays, for example, $10 \mathrm{~min}$. This contrasts with amnesic subjects who are typically impaired on DNMS and DMS tasks after delays of only $40 \mathrm{sec}$ between sample presentation and test (Aggleton et al. 1988; Holdstock et al. 1995; Squire et al. 1988). Furthermore, amnesic subjects show significantly faster rates of forgetting over these relatively short delays (Holdstock et al. 1995), whereas monkeys with selective hippocampectomy do not. These differences suggest that hippocampal damage in monkeys is not sufficient to reproduce the recognition deficit typically found in amnesia.

A final factor concerns the type of stimulus being tested. Studies with rats have shown that both fornix transection and hippocampectomy can disrupt recognition when large, relatively featureless stimuli (test boxes) are used instead of trial-unique discrete objects (Cassaday \& Rawlins 1995; Rawlins et al. 1993). This impairment is most evident when the plain boxes are used repeatedly within a session, that is, are not trial unique (Rawlins et al. 1993), but deficits are also observed when discrete objects are placed in these large test boxes (Cassaday \& Rawlins 1997). A plausible explanation of these results is that the animal encodes the large box or the stimuli inside the large box as part of a spatial (scenic) array rather than as a discrete stimulus (Cassaday \& Rawlins 1995), thus rendering it sensitive to hippocampal dysfunction. When the boxes are small they are encoded as objects, and no deficit is seen (Cassaday \& Rawlins 1997). Similarly, studies with monkeys have shown that fornix lesions can reliably disrupt the recognition of "scenes" in which common elements are repeated but oc- cur in different spatial configurations (Gaffan 1991; 1992b; 1994b). These impairments can be directly related to the widely accepted view that the hippocampus is vital for the efficient encoding of allocentric space (O'Keefe \& Nadel 1978). The importance of stimulus type is further emphasized by the spontaneous orientation task used by Honey et al. (1998) and by recent activation studies (see sect. 5.4).

\subsection{Does testing recognition provide a valid assay for anterograde amnesia?}

A closely related debate concerns whether tests such as DNMS and DMS are a valid assay for amnesia. One view is that recognition is an integral part of declarative memory (Haist et al. 1992; Knowlton \& Squire 1995) insofar as people can subjectively evaluate their memory and either retrieve items (recall) or make judgements regarding their previous occurrence (recognition). This model tightly links the two processes and so predicts that anterograde amnesia will impair both recall and recognition and that the deficits will be related. Alternate views hold that recognition and recall depend, in part, on different processes. One such view is that recognition benefits from an additional component of processing that is based on "perceptual fluency" or "feelings of familiarity" (Gardiner 1988; Gardiner \& Parkin 1990; Jacoby 1991; Mandler 1980; see sect. 6). This process is regarded as being additive to and separate from the explicit memory of an event (Mandler 1980) and corresponds to feelings of "knowing" that something is familiar rather than "remembering" (i.e., recalling) its previous occurrence (Gardiner 1988; Gardiner \& Parkin 1990). As a consequence it may be predicted that a loss of episodic memory need not be accompanied by a comparable loss of recognition.

In fact, a number of reports have described individual amnesic cases (Dusoir et al. 1990; Gaffan et al. 1991; Hanley et al. 1994; Parkin \& Hunkin 1993; Parkin et al. 1993) or even groups of amnesics (McMackin et al. 1995; Volpe et al. 1986) with relatively well-preserved recognition. For example, a group of subjects with bilateral fornix damage following third ventricular cysts (McMackin et al. 1995) was able to perform the RMT tasks within normal limits, even though they were clearly impaired on tests of episodic memory. Individual cases of interest include a man who suffered bilateral traumatic injury to the mamillary body region (Dusoir et al. 1990), in whom PET studies revealed additional hypoactivity in the left hippocampus (Kapur 1995). A clear and persistent anterograde amnesia developed, yet he performed well within normal limits on a series of recognition tests including the RMT (Dusoir et al. 1990). He also performed very well on a DMS task using single abstract patterns (Holdstock et al. 1995; Fig. 3). This is of interest in that the task avoided ceiling effects even though it used a DMS procedure to assess the retention and recognition of single stimuli. Furthermore, all of the other amnesic subjects tested on the same DMS task were markedly impaired, even though their delayed recall deficits (as measured by the Wechsler Memory Scale Revised; WMSr) were comparable to those of the mamillary body case (Holdstock et al. 1995).

Other individual cases include a person who had suffered a hypothalamic tumour close to the mamillary bodies and who displayed a severe anterograde amnesia (Parkin \& Hunkin 1993). This patient achieved scores in the 83rd 
Aggleton \& Brown: Episodic memory, amnesia, and hippocampus

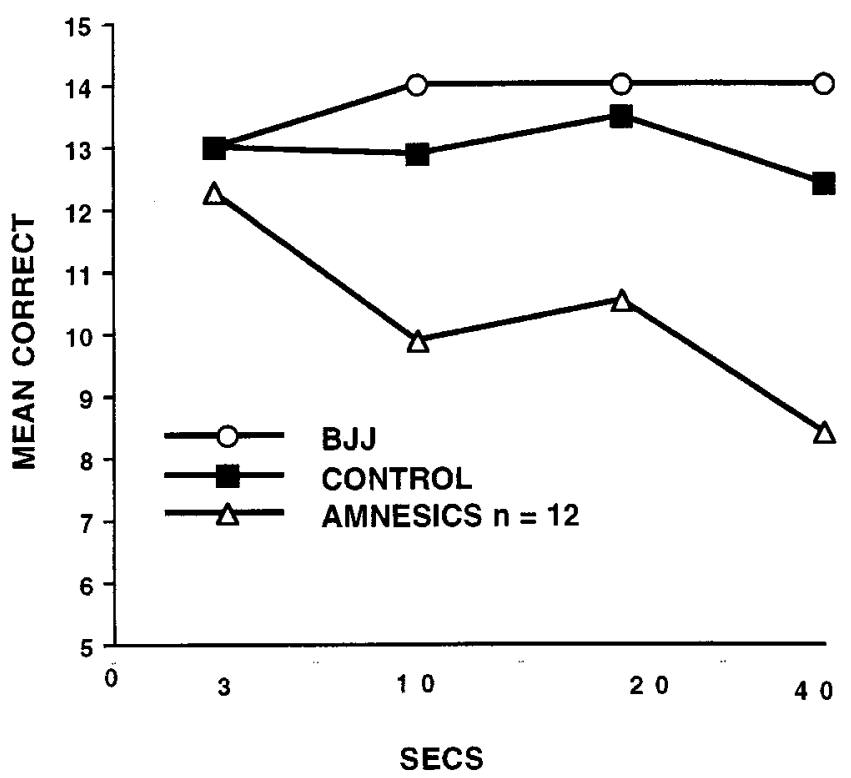

PATTERN RECOGNITION

Figure 3. Performance of an amnesic subject with bilateral mamillary body damage (BJJ) showing spared performance compared to normal controls and a group of mixed amnesics on a delayed matching-to-sample task using visual patterns (data from Holdstock et al. 1995).

(words) and 94th (faces) percentiles on the two RMT subtests (in stark contrast to a score of 56 on the WMSr Delayed Recall Index). Of similar interest was a young woman who displayed Wernicke's encephalopathy following a relatively brief history of alcoholism (Parkin et al. 1993). She showed a chronic, profound impairment on tests of recall but her recognition memory was remarkably well preserved across a variety of tests. These included the RMT on which she scored in the 75th percentile for both words and faces (Parkin et al. 1993), as well as showing normal performance on a more difficult RMT variant in which the face stimuli were presented upside down.

These examples of spared recognition do not arise simply because tests of recognition are easier to perform than tests of recall, nor because the individual patients suffered only from very mild amnesic syndromes. The former can be excluded because a number of studies have taken special care to preclude ceiling effects (Hanley et al. 1994; Holdstock et al. 1995; Parkin et al. 1993; Volpe et al. 1986). Similarly, differences in the severity of the anterograde amnesia can also be discounted because performance on other memory tests has been carefully documented in individual patients with spared recognition (Aggleton \& Shaw 1996; Dusoir et al. 1990; Hanley et al. 1994; Parkin \& Hunkin 1993; Parkin et al. 1993).

\subsection{Is hippocampal function dependent on afferents from the perirhinal region?}

One of the more surprising aspects of the current model is the supposed extent to which some hippocampal functions are independent of their perirhinal inputs. This appears surprising for at least two reasons. First, there are many direct and indirect connections between the two regions. In- deed, the perirhinal and parahippocampal cortices combined provide approximately two-thirds of the inputs to the entorhinal cortex (Insausti \& Amaral 1987; Suzuki 1996a; Suzuki \& Amaral 1994), which is the cortical gateway to the hippocampus. In addition, the hippocampus projects directly upon the perirhinal cortex and entorhinal cortex; the latter projects, in turn, to the perirhinal and parahippocampal cortices (Saunders \& Rosene 1988; Suzuki 1996; Suzuki \& Amaral 1994). Second, the type of information that appears to be present in the perirhinal cortex (see sect. 5.3) could provide the elemental fragments upon which an episodic "memory system" might operate (Brown 1990; Eichenbaum et al. 1994; Gaffan \& Parker 1996; Squire \& Zola-Morgan 1991). Not surprisingly, both classes of evidence are featured in previous models of medial temporal lobe function (Eichenbaum et al. 1994; Squire \& Zola-Morgan 1991). As a consequence these models predict that perirhinal damage should disconnect the hippo-campus and so mimic the effects of hippocampal removal. For this reason it should not be possible to produce a double dissociation between these two regions.

Recent studies on the perirhinal cortex do, however, suggest that this cortical region has a relationship with the hippocampus different from that proposed in previous models (Eichenbaum et al. 1994; Squire \& Zola-Morgan 1991). Most striking is lesion evidence showing that functions of the hippocampus and the perirhinal cortex can be doubly dissociated from one another. In one study fornix lesions in monkeys produced severe deficits on a spatial discrimination and reversal task (Gaffan 1994a). This accords with previous studies showing the sensitivity of this spatial task to lesions in the hippocampus and mamillary bodies as well as in the fornix (Aggleton \& Mishkin 1985; Jones \& Mishkin 1972; Mahut 1971; 1972). In contrast, perirhinal lesions had no apparent effect on the same spatial task (Gaffan 1994a). The same study also tested recognition for visual scenes and found that on this task the perirhinal lesions produced a severe deficit, whereas the fornix lesions resulted in a much milder impairment (Gaffan 1994a). This double dissociation shows that the perirhinal cortex is not a critical way station for all hippocampal inputs and suggests that the mnemonic contributions of the two regions can differ substantially.

Evidence for a similar double dissociation has recently been uncovered in rats (Ennaceur \& Aggleton 1997; Ennaceur et al. 1996). Although fornix lesions severely impaired tests of spatial working memory (T-maze alternation, radial-arm maze nonmatching, and delayed nonmatchingto-position in a Skinner box), cytotoxic perirhinal lesions had no apparent effect (Table 1). In contrast, only the perirhinal lesions disrupted a test of object recognition (Ennaceur et al. 1996). Although they were not tested simultaneously, lesions of the hippocampus and the anterior thalamic nuclei have been shown in other experiments consistently to disrupt these same spatial tasks (Aggleton et al. 1986b; 1995a; 1996), but have no apparent effect on object recognition (Aggleton et al. 1986b; 1995a). Conversely, large neurotoxic lesions including the perirhinal cortex, postrhinal cortex and area TE impaired object recognition but had no apparent effect on T-maze alternation (Aggleton et al. 1997). This last result is especially surprising in that the postrhinal cortex offers an alternative route for spatial information to reach the hippocampus (Naber et al. 1997). Additional evidence for a double dissociation be- 
Aggleton \& Brown: Episodic memory, amnesia, and hippocampus

tween hippocampal and perirhinal functioning has come from a series of c-fos activation studies (see sect. 5.5). These indicate that exposure to novel visual stimuli increases neuronal activity in the perirhinal cortices but not in the hippocampus (Zhu et al. 1995b; 1996). In contrast, exposure to a novel environment can raise hippocampal activity but not perirhinal activity (Zhu et al. 1997).

These results lead to the prediction that selective damage to the human homologue of the perirhinal cortex will impair some memory functions, including recognition, but need not produce a full amnesia. Kapur et al. (1994) described a person with extensive bilateral damage to temporal neocortex combined with apparent sparing of the hippocampus and amygdala. This pathology spared parts of the entorhinal cortex but appeared to invade much of the perirhinal cortex (Kapur et al. 1994). In spite of some everyday memory difficulties, the patient did not suffer from anterograde amnesia (e.g., his WMSr delayed memory quotient was 99). He did, however, show a retrograde amnesia and a "semantic" memory loss. Furthermore, his recognition performance on the faces test of the RMT was severely impaired although word recognition appeared normal, suggesting a hemispheric difference in the extent of pathology.

Other evidence concerns a woman who suffered bilateral damage to the rostral temporal cortex, while subcortical regions appeared intact (Kapur et al. 1992; see also Markowitsch et al. 1993). She displayed a severe retrograde amnesia but only a very mild loss of new learning. Of special interest was the finding that her performance on the faces subtest of the RMT was impaired, yet on recall tests of visual nonverbal memory her performance was excellent (Kapur et al. 1992). Damage to the parahippocampal gyrus was the best predictor of the recognition memory deficit, but it was not associated with anterograde amnesia. Other relevant evidence comes from a description of five cases with a profound loss of semantic information associated with focal temporal lobe atrophy (Hodges et al. 1992). One of the key features of these subjects with "semantic dementia" was the relative preservation of episodic memory (Hodges et al. 1992). Another striking feature of semantic dementia is the finding that the loss of past autobiographical (episodic) information can show a reverse Ribot effect, that is, relative sparing of recent memories (Graham \& Hodges 1997). This is the opposite of what is seen in amnesic syndromes and in Alzheimer's disease (Graham \& Hodges 1997), and hence points to dissociable functions played by the hippocampal system and its neighbouring cortices.

Further evidence for this dissociation comes from a series of three adolescents who suffered bilateral hippocampal pathology at birth or aged 4 or 9 years (Vargha-Khadem et al. 1997). All three show a clear anterograde amnesia affecting episodic memory, yet, remarkably, they have attained levels of language competence and factual knowledge that are within the low to average range. Not only do they show an apparent dissociation between semantic and episodic memory, they also show evidence of relatively well-preserved recognition (forced-choice) memory in the face of deficient spatial and temporal memory (VarghaKhadem et al. 1997). Although potentially important factors such as developmental reorganisation must be considered, these cases provide further evidence against the view of an interdependent relationship between the hippocampus and the temporal (perirhinal) cortices.

\section{Recent support for the proposed model of hippocampal-diencephalic interactions}

\subsection{Behavioural evidence from lesion studies in animals: Spatial memory and scene memory}

In recent years Aggleton and his collaborators have systematically examined the involvement of various limbic brain sites in the performance of tasks dependent on normal hippocampal function (Table 1). These experiments, which have used rats, have focussed on tests of allocentric spatial memory (O’Keefe \& Nadel 1978). Studies using forced spatial alternation in a $\mathrm{T}$ maze have revealed that normal performance depends on the integrity of the anterior thalamic nuclei, the mamillary bodies, and the cingulum bundle as well as the hippocampus and fornix (Aggleton \& Sahgal 1993; Aggleton et al. 1986b; 1995a; 1995b; 1996; Neave et al. 1997). Furthermore, probe tests have confirmed that normal rats use allocentric cues to solve this spatial alternation task (Aggleton et al. 1996; Neave et al. 1997). Not surprisingly, lesions in these same sites (i.e., the anterior thalamic nuclei, the mamillary bodies, the cingu-

Table 1. Effects of lesions in rats showing double dissociations between spatial memory (DNMP, T-maze, radial arm maze, swim maze) and object recognition (spontaneous object recognition, DNMS) ${ }^{a}$

\begin{tabular}{|c|c|c|c|c|c|c|c|}
\hline & Hippocampus & Fornix & $\begin{array}{l}\text { Anterior } \\
\text { thalamus }\end{array}$ & $\begin{array}{c}\text { Mamillary } \\
\text { bodies }\end{array}$ & $\begin{array}{l}\text { Cingulum } \\
\text { bundle }\end{array}$ & $\begin{array}{l}\text { Medial } \\
\text { dorsal th. }\end{array}$ & $\begin{array}{c}\text { PPR } \\
\text { cortex }\end{array}$ \\
\hline DNMP & $x$ & $x$ & $x$ & $\sqrt{ }$ & $\sqrt{ }$ & $\sqrt{ }$ & $\checkmark$ \\
\hline T-maze & $x$ & $x$ & $x$ & $x$ & $x$ & $\sqrt{ }$ & $\sqrt{ }$ \\
\hline Radial arm maze & $(\times)$ & $\times$ & $x$ & $x$ & $\times$ & $\sqrt{ }$ & $\sqrt{ }$ \\
\hline Swim maze latency & $(\times)$ & $\times$ & $x$ & $(X)$ & $\times$ & $(\sqrt{ })$ & $\sqrt{ }$ \\
\hline $\begin{array}{l}\text { Spontaneous object } \\
\text { recognition }\end{array}$ & - & $\sqrt{ }$ & $\sqrt{ }$ & - & $\sqrt{ }$ & - & $x$ \\
\hline DNMS & $\sqrt{ }$ & $\sqrt{ }$ & - & $\sqrt{ }$ & - & $x$ & $(\times)$ \\
\hline
\end{tabular}

${ }^{a}$ A cross indicates a deficit, a check mark indicates no effect, and a dash indicates that the results have not been reported. All data are drawn from published research in the laboratory of the authors, with the exception of those in parentheses, which are from Kolb et al. (1982), Morris et al. (1982), Mumby and Pinel (1994), and Sutherland and Rodriguez (1989). Abbreviations: PPR, perirhinal/postrhinal cortices. 
lum bundle, and the fornix) can disrupt other spatial tasks thought to tax allocentric spatial processing. These include the radial arm maze and the Morris water maze (Aggleton et al. 1996; Byatt \& Dalrymple-Alford 1996; Neave et al. 1997; Sutherland \& Rodriguez 1989; Warburton \& Aggleton 1999; Warburton et al. 1997; 1998). These deficits are selective, however; lesions in the same sites (i.e., fornix, anterior thalamic nuclei, mamillary bodies, and cingulum bundle) do not disrupt a comparable egocentric spatial task in which the animals are rewarded for turning in a constant direction while allocentric cues became irrelevant (Aggleton et al. 1996; Neave et al. 1997).

By using a standard alternation task, it has been possible to compare the severity of the spatial deficits following various limbic lesions. These comparisons show that the alternation deficit is greatest after lesions in the hippocampus, fornix, and anterior thalamic nuclei and least after mamillary body damage or cingulum bundle damage (Fig. 4). Although some of these comparisons are affected by floor effects, they do serve to underline the importance of the anterior thalamic nuclei. Furthermore, because the anterior thalamic lesion effects are significantly greater than those observed after mamillary body lesions (Aggleton \& Sahgal 1993; Aggleton et al. 1995) the results point to a system subserving allocentric spatial memory that involves the direct hippocampal-anterior thalamic projections as well as the indirect hippocampal-mamillary body-anterior thalamic projections (Fig. 5, Table 1). This is consistent with the finding that complete or near-complete neurotoxic lesions of all three anterior thalamic nuclei produce an impairment

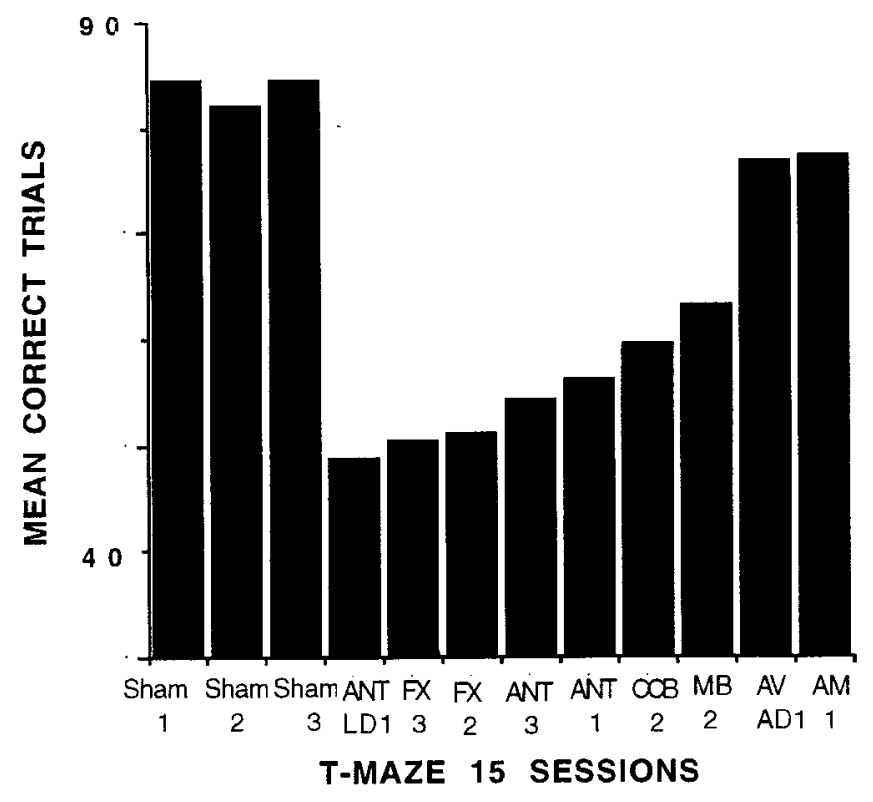

Figure 4. T-maze alternation: Combined summary data from three experiments (No. 1, Aggleton et al. 1996; No. 2, Neave et al. 1997; No. 3, Warburton et al. 1997a) showing the effects of selective limbic lesions on spatial alternation. The histograms show the mean percentage of correct scores for each of the groups over 15 acquisition sessions (90 trials). The lesion locations, from left to right are: sham controls; anterior thalamic nuclei plus lateral dorsal (ANTLD1); fornix (FX); anterior thalamic nuclei (ANT); bilateral cingulum bundle (CCB2); mamillary bodies (MB2); anterior ventral/anterior dorsal thalamic nuclei (AD1); anterior medial thalamic nucleus (AM1). The numbers 1-3 refer to the number of the experiment. as severe as that observed after fornix transection (Warburton et al. 1997).

This focus on the anterior thalamic nuclei raises the question of whether any of the three component nuclei (anterior ventral, anterior dorsal, anterior medial) is especially critical for spatial memory. All three nuclei have substantial connections with the hippocampus, mamillary bodies, and cingulate cortices (Shibata 1992; 1993a; 1993b), although there are some distinct differences in the detailed patterns of these connections. Most notably, the anterior dorsal nucleus receives afferents from the lateral mamillary nucleus, whereas the anterior ventral and anterior medial nuclei receive their afferents from the medial mamillary nucleus (Cruce 1975). The anterior dorsal nucleus also receives the fewest hippocampal inputs (Aggleton et al. 1986a). Single unit recording studies also point to differences within the anterior thalamic nuclei as the distribution of "head direction" cells varies within the anterior thalamic nuclei (Taube 1995), but this has yet to be matched to any particular anatomical boundary or projection zone.

To investigate possible functional differences within the anterior thalamic nuclei the effects of lesions in the anterior medial nucleus have been contrasted with those of more lateral lesions involving both the anterior ventral and the anterior dorsal nuclei (Aggleton et al. 1996; see also Byatt \& Dalrymple-Alford 1996). Both sets of lesions produced mild, but significant, deficits on the T-maze alternation task, but it was only when the lesions were combined that the full effect of anterior thalamic damage became evident. These results not only suggest that all three anterior thalamic nuclei are integral to the proposed system, but also show that attempts to assess fully the effects of anterior thalamic damage should involve all three nuclei. These findings may therefore help to account for those studies in which small, subtotal anterior thalamic lesions had little or no apparent effect on tests of spatial working memory (Beracochea \& Jaffard 1995; Beracochea et al. 1989; Greene \& Naranjo 1986). They also help to explain some of the deficits reported with lesions of the internal medullary lamina following pyrithiamine-induced thiamine deficiency (Langlais \& Savage 1995). This is because damage to the anterior thalamic nuclei and mamillary bodies is a consistent feature of this animal model (Langlais \& Savage 1995) and could account for many of the spatial deficits.

Damage to a number of other sites can disrupt T-maze alternation, including the prelimbic (medial prefrontal) and cingulate cortices (Brito et al. 1982; Markowska et al. 1989; Shaw \& Aggleton 1993; Sutherland \& Hoesing 1993; Sutherland et al. 1988; Thomas \& Brito 1980). Both regions are of interest insofar as they have connections with the hippocampus and anterior thalamic nuclei as well as with the medial dorsal nucleus of the thalamus. Although both cortical regions presumably contribute to the normal processing of these spatial tasks, their importance might have been overestimated. This is because most lesion studies have damaged fibres of passage and adjacent tracts (e.g., the cingulum bundle). With use of cytotoxins to produce selective lesions in these cortical areas, evidence is emerging that even extensive damage to the cingulate cortices has little, if any, effect on spatial tasks such as T-maze alternation (Aggleton et al. 1995b; Neave et al. 1994) or the Morris water maze (Warburton et al. 1998). Similarly, more selective prefrontal lesions often produce only transient deficits on standard tasks thought to assess allocentric spatial memory (Ag- 


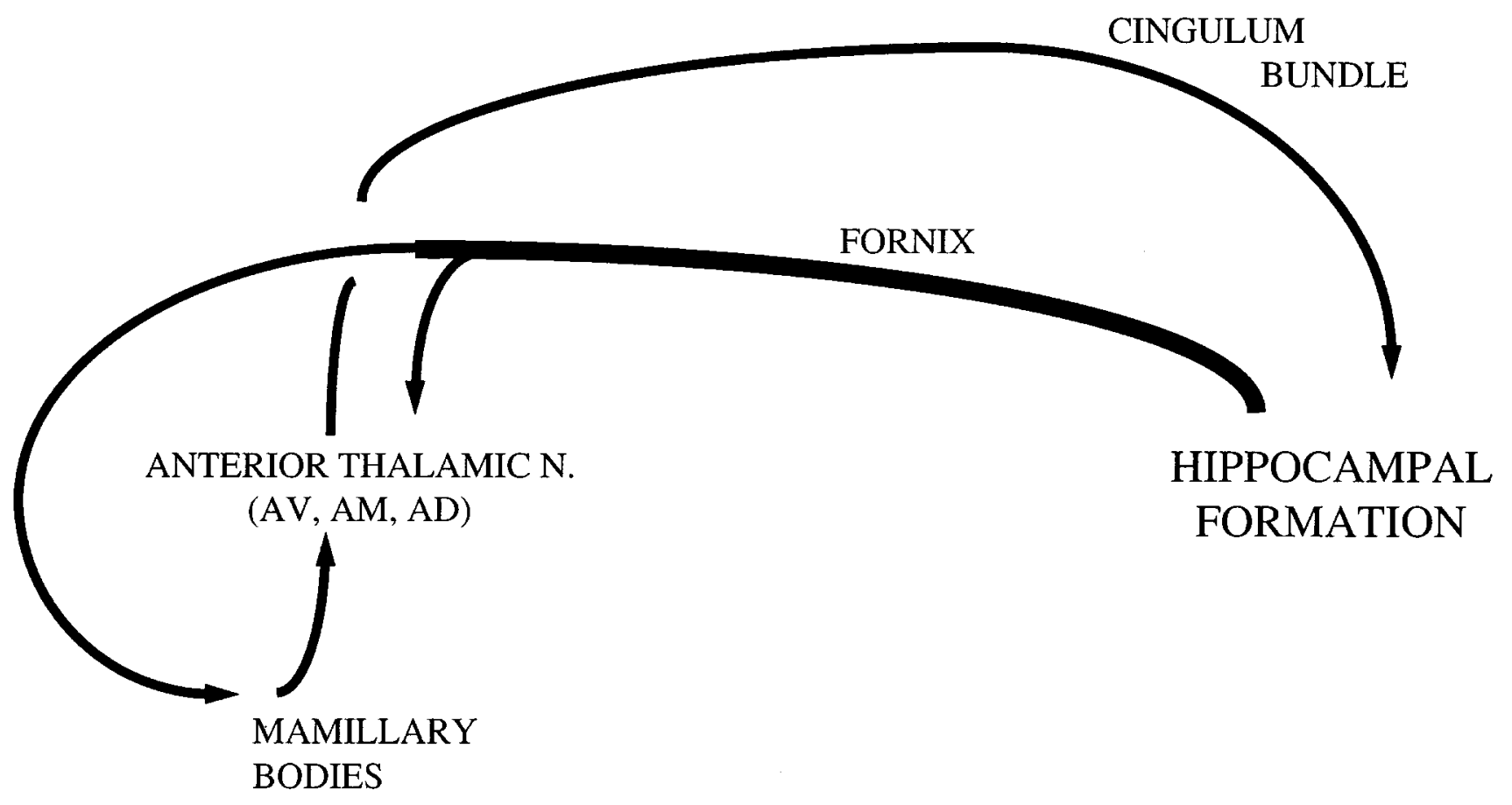

Figure 5. Schematic diagram showing the main group of interconnections underlying pure allocentric spatial memory performance in the rat. The thickness of the line corresponds to the relative importance of the connection as determined by lesion experiments.

gleton et al. 1995b; Shaw \& Aggleton 1993; Thomas \& Brito 1980; Thomas \& Spafford 1984). Furthermore, when more permanent deficits are observed after prefrontal damage, they often appear qualitatively different from those observed after hippocampal damage; for example, they reflect a loss of behavioural flexibility rather than a loss of spatial memory (Aggleton et al. 1995b; Bruin et al. 1994; Granon et al. 1994).

A further diencephalic region that might prove to be of importance is the thalamic midline. Nuclei in this region, most especially the more rostral portions of nuclei such as reuniens, paraventricular, and parataenialis, all have reciprocal connections with the hippocampus. These connections are found across a range of species, although they appear particularly dense in the rat. Although highly selective anterior thalamic lesions show that damage to these midline nuclei is not necessary to induce a spatial alternation deficit (Aggleton et al. 1996), this does not show whether these nuclei contribute to the effects of more extensive lesions. Evidence of a possible involvement of the midline nuclei in the amnesia associated with paramedial thalamic infarcts has come from a PET study showing widespread cortical hypometabolism that might be attributable to the loss of midline nuclei (Levasseur et al. 1992). The contributions of these nuclei clearly require systematic investigation, though this will prove technically difficult.

The studies so far cited have concerned rats or mice; comparable tests of allocentric spatial memory have rarely been conducted with monkeys. It has been shown, however, that fornix lesions impair T-maze forced alternation by monkeys, whereas cingulate gyrus lesions have little or no effect (Murray et al. 1989). Related evidence comes from studies showing that hippocampal and fornix lesions can both impair tasks that require the animal to remember the position of a given object (Gaffan \& Harrison 1989; Parkinson et al. 1988) or to perform a place discrimination and subsequent reversals (Gaffan 1994a; Jones \& Mishkin 1972; Mahut 1972). The effects on such tasks of discrete anterior thalamic lesions have yet to be assessed in monkeys, but the pattern of deficits following lesions in the mamillary bodies (Holmes \& Butters 1983) strongly suggests that spatial deficits will be found. This overall pattern of results is in close accordance with findings from rats.

Other relevant evidence has come from an ingenious series of experiments showing how fornix damage in monkeys disrupts discrimination tasks in which task performance is aided by the ability to remember the spatial disposition of the elements that make up the stimulus. Initial evidence came from the finding that fornix lesions disrupt conditional tasks that tax the ability to identify a particular place (Gaffan \& Harrison 1989). It is important that this deficit was evident only when the places to be distinguished contained common elements that were spatially rearranged (Gaffan \& Harrison 1989). It was proposed that the fornix is necessary for the creation of a snapshot memory that stores the spatial arrangements of the items in a "scene" (Gaffan 1991). This conclusion was supported by the finding that lesions of the fornix will disrupt the acquisition of concurrent discriminations when the stimuli to be discriminated are complex scenes that often contain common elements (Gaffan 1992b; 1994a). This finding has recently been explored in more detail, and it appears that the critical feature is whether task performance (in this case, concurrent discrimination) is aided if the animals can remember the background and location of the stimuli to be discriminated (Gaffan 1994b).

These results have been taken to indicate that the fornix, and hence the hippocampus, is important for the scenespecific memory of objects (Gaffan 1992a; 1994a; 1994b). From use of the same "object-in-place" task there is now evidence that the mamillary bodies are involved in this same process (Parker \& Gaffan 1997a). Furthermore, this 
mamillary body involvement appears to be via its afferents from the fornix (Parker \& Gaffan 1997a). Consistent with the main proposals in this target article, this object-in-place task also depends on the integrity of the anterior thalamic nuclei in monkeys (Parker \& Gaffan 1997b). These studies provide important evidence that the hippocampal-fornixanterior thalamic system might aid the normal recall of episodic information as it permits the subject to distinguish or recreate the unique scene associated with the item to be remembered (Gaffan 1992a; 1994b; Tulving 1983), a process that will reduce interference from other similar events (Gaffan 1994b). Interestingly, surgical ablations of the cingulate cortex produced only a mild impairment (Parker \& Gaffan 1997b) and so echo the effects of selective cingulate/cingulum bundle lesions on spatial memory tasks in rats (Neave et al. 1994; 1996; 1997).

It has been pointed out that the anterior thalamic nuclei and the lateral dorsal nucleus contain "head direction" units (Mizumori \& Williams 1993; Taube 1995). These inform the animal of the direction in which it is pointing, irrespective of actual position in space. In that a loss of thalamic "head direction" information appears to disrupt "place" cells in the hippocampus (Mizumori et al. 1994), this could account for some of the similarities between the effects of damage in these two regions on tests of spatial navigation. This could also explain how anterior thalamic lesions can disrupt the Morris water maze to a greater extent than do fornix lesions (Warburton \& Aggleton 1998), the additional deficit reflecting the loss of head-direction information. This role in providing head-direction information is not, however, sufficient to explain the full array of similarities between the effects of hippocampal and anterior thalamic damage.

Perhaps most important are the findings that lesions of the mamillary bodies and anterior thalamic nuclei in monkeys disrupt the "object-in-place" discrimination task and that this effect is dependent on inputs from the fornix (Parker \& Gaffan 1997a; 1997b). It is difficult to imagine how a loss of head-direction units could account for a deficit in such a discrimination because the animal is pointing to the whole scene. Other evidence comes from the finding that selective lesions of the anterior medial thalamic nucleus in rats are sufficient to disrupt tasks sensitive to hippocampal dysfunction, for example, T-maze alternation or radial-arm maze (Aggleton et al. 1996; Byatt \& DalrympleAlford 1996), even though there is no evidence that this division of the anterior thalamic nuclei contains head direction units (Taube 1995). Single unit recordings also highlight the contribution of the anterior thalamic nuclei to discriminative avoidance tasks that have minimal spatial demands (Gabriel 1993). Finally, studies of neuronal activation in monkeys using the 2-deoxyglucose method have revealed similar increases in activity in the hippocampus, mamillary bodies, and anterior thalamic nuclei on a variety of tests of working memory (Friedman et al. 1990), even though performance on some of the tasks is unlikely to involve head direction information.

\subsection{Reconsidering clinical evidence in light of the proposed model}

A central aspect of the current proposal is that bilateral damage to the fornix, mamillary bodies, or anterior thalamic nuclei is sufficient to induce anterograde amnesia. Furthermore, the more selective the damage, the greater the disparity between the loss of episodic memory and the sparing of recognition.

5.2.1. Fornix damage and amnesia. A highly influential review on the effects of fornix section (Garcia-Bengochea \& Friedman 1987) concluded that damage to this tract was not sufficient to induce amnesia. This review has, however, been strongly criticised (Gaffan \& Gaffan 1991). One problem concerns the need to separate those cases with bilateral and unilateral fornix surgery. This is because unilateral hippocampal ablation in cases of epilepsy does not bring about anterograde amnesia; hence, neither should unilateral fornix section. Gaffan and Gaffan (1991) pointed out that many of the cases regarded as having bilateral fornix sections (Garcia-Bengochea \& Friedman 1987) in fact, had only unilateral surgery. Other problems with this material include the lack of pre- and postsurgical psychometric data. This information is required because some of the relevant cases were psychotic or mentally retarded (Gaffan \& Gaffan 1991; Sugita et al. 1971), making it difficult to measure any change in memory.

There are now a growing number of reports that have linked fornix damage with a loss of episodic memory. Most of them concern the outcome of cysts or tumours in the third ventricle (Calabrese et al 1995; Cameron \& Archibold 1981; Gaffan et al. 1991; Geffen et al. 1980; Heilman \& Sypert 1977; Hodges \& Carpenter 1991; McMackin et al. 1995; Sweet et al. 1959; Tucker et al. 1988). Of special interest is a report by McMackin et al. (1995) in which six patients who had undergone surgical removal of a third ventricular cyst were assessed on a variety of memory tasks. The status of the fornix was examined using MRI, and this revealed a clear association between bilateral fornix damage and a loss of recent verbal and nonverbal memory. This was found even in patients with no signs of ventricular dilation. The only patient to show relatively normal performance on the verbal tests was a patient in whom the left fornix was intact (McMackin et al. 1995). This complements other evidence of an association between left fornical damage and poor performance on tests of verbal memory (Cameron \& Archibold 1981; Hodges \& Carpenter 1991; Tucker et al. 1988). A very small number of reports exist of bilateral fornix damage associated with tumours or surgery for cysts that did not appear to disturb memory (Cairns \& Mosberg 1951; Woolsey \& Nelson 1975), but the impact of these studies is limited by a lack of psychometric information.

5.2.2. Mamillary bodies and amnesia. Although mamillary body pathology has repeatedly been associated with memory loss, it has long been uncertain whether damage in this structure is sufficient to induce amnesia. Initial evidence came from the consistent necrotic state of the medial mamillary bodies in Korsakoff's syndrome (Delay \& Brion 1969; Gudden 1896; Mair et al. 1979; Rigges \& Boles 1944; Victor et al. 1971). This disease typically affects multiple brain sites (Victor et al. 1971), but there are a small number of amnesic cases in which the pathology appeared to have been restricted to the mamillary bodies (Colmant 1965; Delay \& Brion 1969; Remy 1942; Torvik 1987). Caution is required, however, as it is often unclear whether all other regions have been studied (e.g., frontal or temporal lobe areas). Nevertheless, the consistency of mamillary body damage in well-characterised cases of Korsakoff's dis- 
ease is striking (Mair et al. 1979; Mayes et al. 1988). Other relevant evidence comes from reports of amnesia following tumours located in the region of the mamillary bodies (Assal et al. 1976; Benedek \& Juma 1941), but interpretation is hindered by the possible effects of raised intraventricular pressure on other diencephalic regions.

There are a few examples in which pathology appears to be confined to the mamillary bodies, yet no amnesia is reported. The most influential of these negative examples are the five Wernicke cases listed by Victor et al. (1989). These are clearly very important exceptions in that they run counter to the current proposals. Unfortunately no psychometric data for these five cases have been published, leaving it uncertain how it could be determined that their memories were intact (Victor et al. 1989). Furthermore, although Victor et al. (1989) argue that damage to the medial dorsal nucleus of the thalamus is consistently associated with amnesia, other studies have failed to find this precise linkage (Mair et al. 1979; Torvik 1987). In view of the widespread dysfunctions revealed by PET studies of Korsakoff's disease (Fazio et al. 1992; Paller et al. 1997), it is most unlikely that studies of Korsakoff's disease can resolve this issue.

Other evidence comes from the amnesia that can follow thalamic vascular lesions. A consistent feature of the pathology is damage to the mamillothalamic tracts (Castaigne et al. 1981; Cramon et al. 1985; Gentilini et al. 1987; Goldenberg et al. 1983; Graf-Radford et al. 1990; Hodges \& McCarthy 1993; Mori et al. 1986; Parkin et al. 1994; Stuss et al. 1988). This pathway carries projections from the mamillary bodies to the anterior thalamic nuclei and so is integral to the circuitry in this proposal. The thalamic pathology does, however, often invade other regions, including the medial dorsal nucleus and the internal medullary lamina, both of which have also been implicated in memory dysfunction. The conclusions of two surveys that compared the extent of diencephalic pathology and the presence and severity of amnesia are therefore of particular value (Cramon et al. 1985; Gentilini et al. 1987). Both studies failed to find an association with damage to the medial dorsal thalamus (Cramon et al. 1985; Gentilini et al. 1987; see also Daum \& Ackerman 1994; Graf-Radford et al. 1990; Markowitsch 1982). One of these surveys also found no consistent link with internal medullary lamina damage (Gentilini et al. 1987). In all of these studies, however, the presence of bilateral mamillothalamic tract damage was a reliable predictor of amnesia (Cramon et al. 1985; Gentilini et al. 1988).

The corollary of this conclusion is that cases in which the mamillothalamic tract and the anterior thalamic nuclei are spared will not become amnesic. This is supported by the failure of lesions restricted within the medial dorsal nucleus to produce anterograde amnesia (Kritchevsky et al. 1987; Markowitsch 1982). Exceptions to this prediction are case 5 of Castaigne et al. (1981) and a single case described by Calabrese et al. (1993). In the latter case the pathology is based on CT scans (Calabrese et al. 1993) and hence may lack sufficient resolution. In the former case the thalamic pathology is confirmed by postmortem examination (Castaigne et al. 1981), but the fact that this patient suffered from hypertension and loss of consciousness prior to the infarct raises the possibility of pathology in other key brain sites not reported.

Penetrating brain injuries in the region of the mamillary bodies have been described in two amnesic patients ( $\mathrm{Du}$ - soir et al. 1990; Squire et al. 1989). One of these patients (BJ) appeared to suffer bilateral damage closely confined to the mamillary body region (Dusoir et al. 1990), and although the subject showed some recovery he has a permanent impairment for the recall of verbal and nonverbal material (Dusoir et al. 1990; Kapur et al. 1995). The severity of his amnesia, as measured by the WMSr, is typical for anterograde amnesia (Butters et al. 1988). The nature of BJ's injury does, however, mean that other diencephalic pathways could have been damaged (Kapur et al. 1995). Indeed, a PET study of BJ revealed evidence of unilateral hippocampal hypoactivity in the same hemisphere that received the largest amount of mamillary body damage (Kapur et al. 1995). It is not, however, possible to tell whether this hypoactivity reflects a primary pathology or a secondary response to the mamillary body damage. Taken together, there is still no single example of an amnesic with discrete, bilateral mamillary body damage. Nevertheless, the weight of evidence strongly indicates that damage to this region is sufficient to impair episodic memory.

\subsubsection{The anterior thalamic nuclei and amnesia in hu-} mans. Direct evidence implicating the anterior thalamic nuclei in diencephalic amnesia is very limited. In Korsakoff's syndrome, damage to the anterior nuclei occurs only in about one-third of all cases (Victor et al. 1971), whereas the lateral dorsal nucleus shows degeneration in the majority of cases (Brion \& Mikol 1978; Victor et al. 1971). Although infarcts of the paramedian thalamic arteries often spare the anterior thalamic nuclei, they do consistently deafferent the anterior thalamic nuclei by disrupting the mamillothalamic tract.

More direct evidence comes from a handful of cases with anterior thalamic damage. For example, a recent MRI study described a patient with anterograde amnesia associated with an infarct largely confined to the left anterior thalamic nuclei and the left mamillothalamic tract in whom a loss of verbal memory was most pronounced (Clarke et al. 1994). A PET study of the same subject revealed decreased metabolic rate in the posterior cingulate cortex (Clarke et al. 1994), a result consistent with pathology in the anterior thalamic nuclei. Complementary evidence comes from the case of an amnesic man who suffered a lesion involving the retrosplenial cortex and cingulum bundle (Valenstein et al. 1987). A subsequent PET study revealed evidence of hypometabolism in the thalamus, although the medial temporal region appeared normal (Heilman et al. 1990). It was concluded that the amnesia in this patient was a consequence of the disconnection of anterior thalamic pathways (Heilman et al. 1990). A similar explanation could account for a patient who suffered a circumscribed haemorrhage in the left retrosplenial area and cingulum bundle (Von Cramon 1992). A mild, but perceptible, loss of verbal learning and memory was observed, which disappeared after 6 months (Von Cramon 1992).

Other evidence comes from a person who displayed a persistent impairment in the recall of verbal material, but a sparing of recognition (Hanley et al. 1994). The impairment followed an anterior communicating artery aneurysm, which resulted in left hemispheric damage in the anterior thalamus and caudate nucleus (Hanley et al. 1994). In another informative case, a woman suffered an infarct centred on the anterior thalamic nuclei and the genu of the right internal capsule. She developed a severe anterograde amne- 
sia for verbal and nonverbal information (Schnider et al. 1996), which was characterised by a failure to use contextual information so that her recognition performance was marred by high levels of false positives. Her performance improved dramatically to within normal limits when tested with nonsense words and nonsense designs, stimuli for which contextual information (i.e. remembering) would be of less value but familiarity might be sufficient (Schnider et al. 1996). Although this pattern of performance clearly fits with the current proposals it should be noted that the infarct probably disconnected other potentially important fibre tracts.

Finally, the production of bilateral radio frequency lesions in the anterior nuclei induced a loss of memory in a patient with chronic depression (Mark et al. 1970). It was possible to confirm the placement of the lesions following the suicide of the patient 6 weeks after surgery. The lesion destroyed the whole of the anterior nuclear complex "but essentially no cells of nucleus ventralis anterior or nucleus dorsomedianum" (Mark et al. 1970). Although the patient refused to take memory tests, she expressed concern about her recent memory loss and she had difficulty in remembering the location of her hospital room. Although the authors regarded the memory loss as transient, this could not be verified.

\subsection{Electrophysiological studies}

Neuronal recording studies provide strong support for the idea of a division of function within the medial temporal lobe between the perirhinal and the hippocampal cortices. Indeed, electrophysiological evidence of the importance of perirhinal cortex rather than the hippocampus in judging stimulus familiarity preceded lesion evidence (Brown et al. 1987). A number of studies have now examined neuronal responses in anterior inferior temporal cortex, including perirhinal cortex, during the performance by monkeys of recognition tasks using large sets of stimuli (Brown et al. 1987; Eskander et al. 1992; Fahy et al. 1993a; Li et al. 1993; Lueschow et al. 1994; Miller et al. 1993; Riches et al. 1991; Sobotka \& Ringo 1993; 1994; for reviews, see Brown 1996; Brown \& Xiang 1998; Ringo 1996). The tasks employed have been either variants of DMS or variants of a serial (running) recognition memory task (Gaffan 1974). These studies have confirmed that many neurones in perirhinal cortex respond maximally to first presentations of visual stimuli, but less so to subsequent presentations. Hence, the reoccurrence of a particular stimulus is signalled by a decrease in the neuronal response to that stimulus. Similar responses have been found in the perirhinal cortex of the rat (Zhu et al. 1995a). It should be noted that, in monkeys, neurones that increase their responses when a stimulus reoccurs are found even less often than might be expected by chance (Xiang \& Brown 1998).

These changed responses with stimulus repetition are sufficient to solve recognition memory tasks such as DMS and DNMS as commonly tested in monkeys. Thus: (1) a single exposure to a stimulus is sufficient to cause a change in responsiveness, that is, one-trial learning; (2) for many neurones, the response change is found even when a long period of time (for example, $24 \mathrm{hr}$ ) has elapsed and/or many (hundreds of) presentations of other stimuli have intervened between the first and the next presentation of a stimulus; and (3) the effect is highly stimulus specific; that is, a neurone that responds weakly to a stimulus that has been seen before still responds strongly to novel stimuli, though there may be a limited amount of stimulus generalisation (Brown 1996; Lueschow et al. 1994; Ringo 1996). Moreover, individual neurones independently signal different types of information of potential use to the judgement of prior occurrence (Fahy et al. 1993a; Zhu et al. 1995a). Thus, there are "recency neurones" that encode whether a stimulus has been seen recently irrespective of whether it has been seen many or few times previously. Other, "familiarity neurones" encode the relative familiarity of stimuli (i.e., whether they have been seen many or a few times previously) without regard to whether they have been seen recently. A third category responds best to novel stimuli or first presentations of unfamiliar stimuli that have not been encountered recently (Xiang \& Brown 1997; 1998). All three types of neurones are found in anterior and medial temporal cortex (area TE) and in entorhinal cortex as well as in perirhinal cortex (Xiang \& Brown 1997; 1998). Neurones have been reported ( $\mathrm{Li}$ et al. 1993) whose response changes progressively as initially unfamiliar stimuli are successively repeated (and presumably become increasingly familiar). Other types of neuronal activity changes such as incremental responses (Miller \& Desimone 1994) and increased firing after stimulus offset (delay activity; Fuster \& Jervey 1981) are also seen in these regions, and they may facilitate or provide alternative means of solving specific types of recognition memory tasks (Brown 1996).

Carefully controlled experiments have established that the described response changes are not artefactual: they cannot be explained by alterations in eye or body movements, or motivational or attentional factors (see Brown 1996). Importantly, they are found not only during the performance of recognition memory tasks, but also when stimuli are shown without the animal being required to make a particular behavioural response and using types of stimuli not employed in the animal's training (Brown 1996; Riches et al. 1991). Accordingly, the response changes are an endogenous rather than a training-induced property of the neurones.

Not all visually responsive neurones recorded in perirhinal cortex change their response with stimulus repetition; many respond equivalently even after the same stimulus has been shown many times. The latter type of neurone encodes information concerning the physical characteristics of the stimuli and so may contribute to stimulus identification. Nevertheless, the incidence of neurones whose responses do change is about $20-25 \%$ of the whole neuronal population (Brown 1996), a finding that has been exploited in c-fos studies (see sect. 5.4). Neurones with changing and neurones with unchanging responses are found intermingled, so comparisons between cells with the two types of response could provide information concerning the previous occurrence of a stimulus. Furthermore, as the responsiveness of both types of neurones varies with the physical characteristics of the stimulus, and the degree of response decrement and the time for which it lasts vary amongst neurones whose responses change, population measures can potentially provide a sensitive measure of the past occurrence of a particular stimulus (both of its recency of occurrence and of its familiarity).

There is also electrophysiological and ablation evidence that the perirhinal region is involved in the learning of visual paired associates (Higuchi \& Miyashita 1996; Miyashita et al. 1996; Murray et al. 1993; Okuno \& Miyashita 
1996). Thus electrophysiological evidence supports a role for perirhinal cortex in recording knowledge concerning individual stimuli and of simple associations between such stimuli. Information concerning the prior occurrence of individual stimuli is also available in the inferior temporal cortex adjacent to perirhinal cortex, but in that region it is likely to be restricted to the visual modality. Perirhinal cortex receives multimodal sensory input (Burwell et al. 1995) and so may serve a similar function for information not confined to a single modality, although present evidence is largely confined to the visual modality. Information about prior occurrence is also available within entorhinal cortex (Fahy et al. 1993b; Suzuki et al. 1995; Xiang \& Brown 1998). In contrast, neurones that change their response after a single occurrence of an individual stimulus are uncommon in the hippocampus of monkeys (Brown et al. 1987; Miyashita et al. 1989; Riches et al. 1991; Rolls et al. 1993; Xiang \& Brown 1998) and rats (Zhu et al. 1995a), a finding confirmed in cfos studies in the rat (Wan et al. 1997; Zhu et al. 1995b; 1996). Thus such perirhinal and entorhinal information is not necessarily passed on to hippocampal neurones in spite of the numerous interconnections between these regions.

Contrasting with the findings for individual stimulus repetition is much evidence that the activity of hippocampal neurones reflects features of the spatial environment or spatial arrangements of stimuli (Eichenbaum et al. 1994; Eifuku et al. 1995; Gothard et al. 1996; O'Keefe 1993; O’Keefe \& Nadel 1978). In particular, individual neurones increase their firing when a rat is located in a particular part of an environment ("place fields"). Such place fields are influenced by the presence of defining features of that environment, including its size and shape (O'Keefe \& Burgess 1996). In the monkey, increases in firing have been recorded when the animal directs its gaze to a particular part of the environment ("view fields"; Rolls \& O’Mara 1995). Additionally, changes in hippocampal responses related to a combination of the relative familiarity of a stimulus and the place in which it was shown have been described (Miyashita et al. 1989), as they have in entorhinal cortex (Suzuki et al. 1995). Although hippocampal neurones also respond to discrete stimuli and during paradigms that are not spatial (see, e.g., Brown 1982; Eichenbaum et al. 1994; Eifuku et al. 1995; Otto \& Eichenbaum 1992b; Thompson 1990), electrophysiological recordings have not yet established that the response characteristics of hippocampal neurones could provide a satisfactory explanation for the properties of episodic memories. Spatial information is important to episodic memory, but it does not encompass the whole of the information contained in the context and content of all episodic memories. Nevertheless, the anatomical connectivity of the hippocampus is ideal for making the widely distributed associations (Brown 1990; Lorente de No 1934) between the many different, contemporaneously experienced stimuli making an event. Such associations are necessary to the formation of an episodic memory.

Recordings in the monkey medial thalamus have revealed neurones whose responses signal information concerning the prior occurrence of stimuli. Neurones in both the medial dorsal nucleus and the paraventricular midline nucleus responded more strongly to first presentation than to subsequent presentations of stimuli during the performance of a recognition memory task (Fahy et al. 1993b). Such findings are consistent with the suggested functional link between perirhinal cortex and the medial dorsal nu- cleus of the thalamus, although the numbers of such neurones were small both in absolute and in percentage terms. There are no reported recordings from the anterior thalamic nuclei during the performance of recognition memory tasks. However, neuronal responses in the anterior thalamic nuclei in the rat can code spatial information, particularly about the direction of the head (Mizumori \& Williams 1993; Taube 1995). Thus, thalamic recordings again support the apportionment of function between the hippocampal-anterior thalamic and the perirhinal-medial dorsal thalamic axes, with the latter underlying the detection of the prior occurrence of a stimulus (its sense of familiarity, "knowing" that something has occurred previously) and the former being involved in the processing of important components, including the contextual information concerning events as is necessary for "remembering" or "recollecting" a prior occurrence.

\subsection{Brain activation studies: $c$-fos}

The expression of the immediate early gene c-fos provides a potential marker for changes in neuronal activity (Herdegen 1996) and so can be used to identify regions that respond to specific experimental manipulations. We have used this technique to help map brain regions involved in recognition (Zhu et al. 1995b; 1996). In the first of these studies, counts of fos-stained nuclei were compared in the brains of rats shown novel objects and in the brains of rats shown highly familiar objects (Zhu et al. 1995b). The objects were placed behind a glass screen, limiting the task to visual processes. Four of the eight sites examined showed significantly higher levels of fos protein in the brain exposed to novel stimuli. These sites were the occipital cortex, area TE, perirhinal cortex, and anterior cingulate cortex. No differences were observed in the entorhinal cortex, the diagonal band of Broca, the medial dorsal nucleus of the thalamus, or the hippocampus (Zhu et al. 1995b). These cortical sites of increased activation correspond closely to those described in a human PET study comparing exposure to familiar and unfamiliar visual stimuli (Vandenberghe et al. 1995). Increased blood flow was found in the lateral anterior temporal neocortex, the medial temporal pole, and the anterior cingulate cortex following exposure to the unfamiliar stimuli (Vandenberghe et al. 1995).

A shortcoming with the c-fos result concerns the need to control for any differences in behaviour induced by the presence of novel objects (e.g., eye movements, alertness). For this reason a second study used a within-subjects design (Zhu et al. 1996). On each trial two objects were shown simultaneously to the rat, so that one eye saw only novel objects while the other eye saw only familiar objects (Fig. 6). This "paired-viewing" procedure makes it possible to compare fos levels in the two hemispheres of the same brain. Novel stimuli activated significantly more neurones (as measured by fos) in the perirhinal cortex, area TE, and ventral lateral geniculate nucleus of the thalamus. No differences were observed in the hippocampus or any other area sampled. Moreover, the number of stained (activated) neurones in the hippocampus was low.

The failure of novel stimuli to evoke significantly increased c-fos products in the hippocampus might be because neurones in this structure do not show activity-related expression of c-fos. This possibility was excluded by a third study (Zhu et al. 1997), in which exposure to novel ob- 


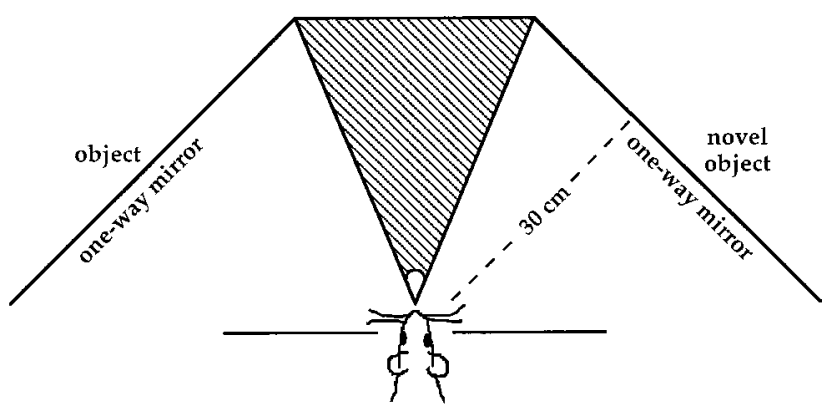

Figure 6. Testing arrangement for the c-fos "paired-viewing" procedure, in which one hemisphere receives direct visual information from an eye seeing novel stimuli while the other eye views familiar stimuli (Zhu et al. 1996).

jects took place in either a familiar or a novel context. Relative to the perirhinal cortex, the hippocampus (fields CA1-4) showed a fourfold increase of activity for the novel context compared to the familiar context. This result, which contrasts with that for novel compared to familiar objects in perirhinal cortex, provides a double dissociation between the hippocampus and the perirhinal cortex. As a consequence the result concurs with lesion and electrophysiological studies pointing to a qualitative difference between the contributions of the two regions to recognition and contextual (spatial) memory.

To explore this difference further a subsequent study compared c-fos activation when rats were exposed either to novel or familiar visual stimuli or to novel or familiar spatial rearrangements of familiar stimuli (Wan et al. 1999). As in previous studies the former condition led to higher c-fos expression for novel than for familiar stimuli in the perirhinal cortex but not in the hippocampus or postrhinal cortex. In contrast, the novel spatial compared to familiar spatial rearrangements resulted in increased c-fos activation in the postrhinal cortex, and, whereas some regions of the hippocampus showed increased (CAl) activation, others (dentate gyrus and subiculum) showed decreased activation (Wan et al. 1999). In view of the supposed homology between the postrhinal cortex and the parahippocampal gyrus (Burwell et al. 1995), these findings provide an insight into how novelty per se need not engage the hippocampus, but novelty arising from the mismatch of certain learnt associations in space may do so. This conclusion matches that found in lesion studies of novelty detection (Honey et al. 1998) and conditional learning (Gaffan \& Harrison 1989). It also accords with a recent functional MRI study showing raised activation in the parahippocampal gyrus during the presentation of novel words or scenes but increased activation in the subiculum during retrieval (Gabrieli et al. 1997).

\section{The consequence of multiple process models of recognition}

Until now recognition has largely been considered as though it were a single process, but many psychologists argue that it involves at least two distinct processes (Gardiner \& Parkin 1990; Jacoby \& Dallas 1981; Mandler 1980). One of these permits a recognition judgement to be made on the basis of stimulus familiarity (sometimes regarded as "perceptual fluency"). Using this information, subjects will have a feeling of "knowing" that they have experienced the test item but may remember no other associated details. The second process is regarded as elaborative; it involves recollecting ("remembering") the experience of the test item. As a consequence the item may be recalled along with associated contextual information (Perfect et al. 1996). Familiarity is regarded as an automatic process, whereas recollection is an effortful retrieval process. An alternative view of recognition is that "know" responses merely reflect a weaker recognition process than "remember" responses and that they are part of the same mechanism.

Evidence for this two-process account comes from manipulations, such as changing levels of processing and increasing the interval between sample and test, that differentially affect the two classes of response (Gardiner 1988; Rajaram 1993; Tulving 1985a). Most importantly, there are conditions that affect "know" responses but not "remember" responses, and vice versa, producing a double dissociation between the two classes of response (Gardiner \& Java 1990; Rajoram 1993). In spite of these dissociations, it has been argued that a two-process model might not be necessary and that it is possible to model these dissociations using a single process model within which different criteria are set for "remember" and "know" responses (Donaldson 1996; Hirshman \& Master 1997). It should also be noted that some of this debate relates not so much to the notion of a distinction between recollective and nonrecollective memory but more to the way that recognition memory is measured (Donaldson 1996). Furthermore, recent analyses of receiver operator characteristics (ROCs) provide further support to dual-process models (Yonelinas 1994; 1997). This final approach is of special interest in that it has led to a comparison (Yonelinas 1997) of recognition judgements for item information and associative information (learned pairs). This revealed evidence that item recognition and associative recognition rely differentially on recollection and familiarity. Whereas item recognition reflected principally familiarity but also recollection, the recognition of associative information relied on recollection (Yonelinas 1997). This distinction is especially relevant because it maps onto the proposed differences between the contributions of the perirhinal cortex to recognition (item familiarity) and the hippocampus to recognition (associative recollection).

It is therefore assumed that the hippocampal-anterior thalamic system supports recollective-based recognition (remembering) whereas cortical (perirhinal) systems support familiarity-based recognition (knowing). As a consequence, all amnesics will show a marked fall in recollectivebased recognition although the loss of familiarity-based recognition will vary according to the extent of cortical (extrahippocampal) dysfunction. Thus, very selective hippocampal system damage may spare those tests of recognition that can be performed effectively by using just relative familiarity. In contrast, more extensive cortical damage, as found in the majority of cases, will disrupt both processes. The proposal that the perirhinal contribution to this form of memory is distinct from that of the hippocampus (because double dissociations can be found) has further implications. Namely, that recollection could occur without familiarity. There is good evidence that this can indeed occur (Jacoby \& Dallas 1981; Jones \& Gardiner 1990; Mandler 1980), but it would be expected to be unusual given the interlinked flow of afferent sensory information to the rhinal region and hippocampus (Gaffan \& Parker 1996; Mishkin 
\& Murray 1994; Suzuki 1996b). From this proposal it can be seen that the two components of recognition are regarded as distinct processes that are independent of one another ( Jacoby 1991; Yonelinas \& Jacoby 1995) but not mutually exclusive. This latter point is important; tests based on "remember" and "know" decisions carry with them an assumption that the processes are mutually exclusive (subjects can never respond both "know" and "remember") and for this reason provide an imprecise measure of the two processes. This is because "know" responses reflect familiarity in the absence of recollection, whereas "remember" responses will contain some items that are both recollected and familiar (see also Donaldson 1996).

One method for testing these possible dissociations within memory has been to assess whether manipulations that lead to similar levels of recognition in amnesics and controls also result in matched levels of recall. In one study Korsakoff subjects received an extended study time so that their word recognition performance was equated with that of control subjects (Hirst et al. 1986). The same extended period of study did not, however, abolish the recall deficit, and it was concluded that recall and recognition are differentially affected by amnesia (Hirst et al. 1986). A second study equated recognition performance in amnesics and controls by testing the controls with longer retention delays (Hirst et al. 1988). At the same delays, the recall performance of the amnesics was still significantly poorer than that of the controls (Hirst et al. 1988). Similar studies have since been repeated, however (Haist et al. 1992), and they failed to show a difference between recall and recognition. This led to the conclusion that recall and recognition are tightly linked functions of declarative memory (Haist et al. 1992). The difficulty with these experiments is that they treat recognition as a unitary process and assume that equating groups across different retention delays is valid. One problem is that "know" and "remember" responses have differential rates of forgetting (Tulving 1985a), limiting such comparisons.

A second study (Knowlton \& Squire 1995) used the know/remember distinction to examine recognition performance in a group of 13 amnesics. The amnesics showed a significant loss of both remember and know responses but revealed a disproportionate loss of remember responses compared to control subjects tested with the same retention delay. Although this pattern is consistent with the present proposals, the same study did include some amnesics with relatively selective pathology. The study reported that these cases did not show a different profile of impairment (Knowlton \& Squire 1995) as might be predicted (but note the debate over anoxia and covert pathology; sect. 4.1). It is the case, however, that the data for these individual subjects were not provided, and shortcomings of the know/ remember distinction have already been discussed.

Single-case information comes from an atypical Wernicke-Korsakoff subject who showed remarkable sparing on tests of recognition (Parkin et al. 1993), suggestive of a selective loss of recollective-based recognition. She was tested using the know/remember subjective distinction, and, although she produced the predicted increase in know responses, she showed only a very modest drop in remember responses. Other evidence comes from an amnesic patient whose performance on different tests of recognition, following anterior communicating artery aneurysm, was best described by the extent to which familiarity informa- tion could be used (Parkin et al. 1994). Although this case supports the current proposals, it was not possible to determine the locus or extent of the brain injury. Other support comes from studies showing that the recognition performance of Korsakoff amnesics depends on judgements of trace strength, that is, familiarity (Huppert \& Piercy 1978).

Taken together, these studies provide general support for the current proposals, but they fail to reveal the pattern of recognition loss following selective hippocampal system damage. The finding that amnesics with selective pathology can perform the Warrington Recognition Memory Test (RMT) within normal limits (Aggleton \& Shaw 1996) accords with the present views only if it is assumed that the RMT can be performed accurately using just familiarity information. Some support for this assumption comes from the discovery that performance of the Faces Subtest of the RMT (the Words Subtest was not examined) is not influenced by extreme switches in context between the sample phase and the test phase (Parkinson \& Aggleton 1994) even though the same switches markedly impair recall (Godden \& Baddeley 1975; Martin \& Aggleton 1993) and, hence, should disrupt recollective processes.

At this point it is necessary to consider the relationship between familiarity and priming, particularly repetition priming. Because familiarity (a feeling of "knowing") is an essentially explicit, conscious form of memory, whereas priming is fundamentally implicit, these two processes cannot have identical substrates; that is, "know" responses cannot be a direct consequence of priming mechanisms and nothing else. Nevertheless, many descriptions of "knowing" regard it as reflecting increased perceptual fluency, that is, priming. Moreover, in the present model familiarity as well as priming is regarded as an automatic consequence of passive exposure to stimuli. Accordingly, processes leading to priming and to familiarity will normally co-occur, yet the two can be dissociated. Thus, priming is often preserved in amnesics even though they are typically poor at tests of recognition (Cermak et al. 1985; Hamann \& Squire 1997; Schacter et al. 1991; Warrington \& Weiskrantz 1974), including the RMT. This distinction is highlighted in an amnesic, EP, who showed chance performance on a range of recognition tasks (i.e., could not use familiarity information) yet displayed intact priming on matched tasks (Hamann \& Squire 1997). Other evidence comes from the loss of know responses in many amnesics (Knowlton \& Squire 1995), which contrasts with the preservation of other forms of implicit memory.

These findings leave open the possibility that priming and familiarity share the same initial stages of processing but that familiarity requires additional, separate mechanisms that give rise to subjective appreciation ("knowing") and allow explicit guidance of choice behaviour. Two further possibilities concerning these additional mechanisms are suggested by the data available. One is that processing involving the integrity of perirhinal cortex is necessary for familiarity but not for priming: this is consistent with the studies reviewed above, including those of patient EP (Hamann \& Squire 1997). The other requires that the absence of contextual information (remembering) renders very difficult the use of primed information to aid recognition, even though priming is intact as measured by other means. However, this second suggestion leaves unanswered the problem of why this implicit information cannot be used even in a forced-choice paradigm (Hamann \& Squire 1997). Similarly, it does not explain why monkeys with hip- 
pocampal system damage can still perform at normal or near-normal levels on DNMS tasks, nor how some amnesics can perform within normal limits on certain tests of recognition (insofar as both have lost contextual information). Thus, it seems necessary to adopt the former of these suggestions and conclude that, unlike the case with repetition priming, feelings of familiarity depend on processing involving perirhinal cortex (Wagner et al. 1998).

This conclusion may well be relevant to a study using the "process-dissociation procedure" (Jacoby 1991), which is designed to separate recollective (explicit) processes from implicit aspects of recognition. It was found that amnesics showed a disproportionate loss in the use of recollection as a basis for recognition (Verfaellie \& Treadwell 1993). This component of the study supports the current proposals; such a loss would reflect the core loss of episodic memory. The amnesic subjects were, however, as likely as control subjects to use perceptual fluency (Verfaellie \& Treadwell 1993). This latter finding is also consistent with the current proposals if the measurement of "familiarity" in the processdissociation procedure corresponds to implicit memory (which may be spared). If, on the other hand, this measure reflects perirhinal familiarity, then the results are at odds with the current proposals, unless the pathologies were unusually selective. It should also be added that the findings of Verfaellie and Treadwell (1993) have been challenged (Roediger \& McDermott 1993; but see Verfaellie 1994), as has the validity of the process-dissociation procedure (Dehn \& Engelkamp 1997; Ratcliff et al. 1995).

A final consequence of the multiple process model of recognition is that it can unify different descriptions of the deficit that follows selective hippocampal-anterior thalamic damage. The current proposals argue that such damage will spare familiarity-based recognition but impair recollection-based processes in recognition and recall. This will result in a loss of associative memory (Yonelinas 1997) and, hence, source memory. This prediction is supported by a study confirming that contextual knowledge for items in a recognition test is consistently higher for "remember" items than for "know" items (Perfect et al. 1996). Thus the current proposals are consistent with those of Gaffan (1991; 1992a; 1994b), that is, that the hippocampus-anterior thalamic axis is required for the creation of episodic (associative) scenes that can heighten discriminability and so aid retrieval of the item to be remembered.

\section{Diencephalic amnesia and recognition memory}

Amnesia associated with diencephalic damage typically results in a severe loss of recognition. That there is a loss of both familiarity and recollective-based recognition is therefore assumed. Whereas the recollection deficit arises from dysfunctions in the hippocampal-anterior thalamic system, the source of the familiarity deficit must be elsewhere. Experimental evidence from behavioural studies of both monkeys (Aggleton \& Mishkin 1983b; Parker et al. 1997; ZolaMorgan \& Squire 1985a) and rats (Hunt \& Aggleton 1991; Mumby et al. 1993) points to the importance of the medial dorsal nucleus of the thalamus. Lesions in this region impair DNMS acquisition and performance, which heavily taxes familiarity judgements. Cells in the medial (magnocellular) portion of the medial dorsal nucleus and in the adjacent midline nuclei show decremental responses to fa- miliar visual stimuli (Fahy et al. 1993; see sect. 5.3). Moreover, the medial dorsal nucleus is very often affected in diencephalic amnesic syndromes; gliosis is consistently observed in Korsakoff's disease (Victor et al. 1971), whereas paramedial thalamic infarcts disconnect both frontal and temporal interactions (Graf-Radford et al. 1990; Malamut et al. 1992). There have been two cases of lesions largely confined within the medial dorsal nucleus that had no apparent effect on memory (Kritchevsky et al. 1987), but these involved only about $15 \%$ of the nucleus and so do not provide a conclusive test of the present proposals.

The medial dorsal thalamic nucleus also receives inputs from the perirhinal cortex. Direct projections run to the magnocellular portion of the medial dorsal nucleus via the inferior thalamic peduncle (Aggleton et al. 1986a; Russchen et al. 1987). In light of the current proposals, it is noteworthy that these connections do not use the fornix, nor does the perirhinal cortex appear to project to the anterior nuclei of the thalamus (Aggleton \& Saunders 1997). The magnocellular portion of the medial dorsal nucleus has dense projections to the medial and orbital frontal cortices, and removal of these cortical regions also impairs DNMS performance in monkeys (Bachevalier \& Mishkin 1986). In contrast, removal of the dorsolateral prefrontal cortex, which receives its inputs from the lateral (parvocellular) portion of medialis dorsalis, does not affect DNMS performance (Bachevalier \& Mishkin 1986). Furthermore, lesions of the uncinate fascicle, which connects the temporal association cortex with the prefrontal cortex, have no effect on DMS (Gaffan \& Eacott 1995). These findings all suggest that one set of key perirhinal outputs for recognition are those to the thalamic nucleus medialis dorsalis (Parker et al. 1997) and thence to the prefrontal cortices. These perirhinal outputs are unlikely, however, to be the sole route; the magnitude of the medialis dorsalis recognition deficit appears less than that associated with perirhinal damage (Aggleton \& Mishkin 1983b; Parker et al. 1997). This indicates that other perirhinal outputs contribute to recognition but that none of these other routes is individually critical. Candidates include the projections to prefrontal cortex to entorhinal cortex (Meunier et al. 1993) and those back to the inferior temporal cortex (in the case of visual recognition).

In view of the fact that the major ouput from medialis dorsalis is to the prefrontal cortex, it is relevant that frontal damage in humans can sometimes disrupt recognition and also that this is not an invariable finding (Aggleton \& Shaw 1996; Schacter 1987; Shimamura et al. 1990). This is highlighted by a recent survey (Wheeler et al. 1995) concluding that frontal lobe damage could impair recognition, although the effects are smaller than those for free recall. It should be noted, however, that dorsolateral prefrontal lesions in monkeys do not disrupt DNMS performance, and it is this region that is involved in many of the human cases. Clinical cases with extensive medial and orbital damage are rarer, and it is possible that such damage is sufficient to impair recognition consistently. The relative mildness of the frontal recognition impairment might also reflect the presence of projections from the medial dorsal nucleus back to the rostral perirhinal cortex (Markowitsch et al. 1985) that might aid recognition. The mild frontal deficit in the judgement of previous occurrence can be contrasted with the more robust failure to discriminate relative recency or source information (Schacter 1987; Shimamura et al. 1990). Evidence that recency judgements and recognition judge- 
ments are not based on the same information has been reported elsewhere (Brown 1996; Shaw \& Aggleton 1995), and taken together these findings suggest that, although the prefrontal cortex may receive information concerning familiarity (either directly from the temporal lobes or from the thalamus), this need not be critical for recognition. Indeed, recent PET studies provide evidence of a dissociation between temporal lobe and dorsolateral prefrontal activity, with temporal lobe activity highest for novel stimuli and prefrontal activity highest for novel rearrangements of familiar stimuli (Dolan \& Fletcher 1997; see also Tulving et al. 1996).

The prefrontal cortex (like the entorhinal cortex) is, in fact, in a privileged position in that it receives both familiarity information and source information. This source information is presumably associated with the direct inputs to the prefrontal cortex from the hippocampus, along with inputs from the medial portions of the thalamus, including the anterior thalamic and midline nuclei (Kievit \& Kuypers 1977). Brain activation studies also indicate that the frontal lobes have a specific involvement in recollective (Dolan \& Fletcher 1997; Wilding \& Rugg 1996) and retrieval (Rugg et al. 1996) aspects of recognition. The key difference is that frontal damage does not reproduce the temporal lobe or diencephalic amnesic syndromes, so its involvement is often not critical. To account for this it is assumed that the hippocampal-anterior thalamic axis has both a diffuse frontal extension as well as an important reciprocal component from the thalamus back to the temporal lobe and hippocampus. Activity in the latter system is sufficient to ameliorate some of the effects of prefrontal damage on standard tests of recall and associative recognition. At the same time, the prefrontal cortex allows the most effective recall strategies to be employed.

\section{Subdivisions of amnesia}

The present proposals run contrary to the traditional division between temporal lobe and diencephalic amnesia and predict that the core symptoms should appear very similar. Indeed, when the problems of matching the severity of the amnesia among different cases and allowing for differential damage to additional structures (e.g., frontal cortex) are taken into consideration, the evidence for qualitative differences in the core features of these amnesias seems weak (Weiskrantz 1985; Zola-Morgan \& Squire 1993). Initial evidence of differences in forgetting rates between temporal lobe and diencephalic amnesia (Huppert \& Piercy 1979; Squire 1981) failed to survive later scrutiny (Freed \& Corkin 1988; Freed et al. 1987; McKee \& Squire 1992). Although some diencephalic amnesics do show a greater loss of short-term memory and contextual cues (Parkin 1984; Parkin et al. 1990), these differences often relate to Korsakoff's syndrome, which results in frontal dysfunction. This may also account for the loss of short-term memory (Cave \& Squire 1992; Leng \& Parkin 1989). Evidence of a greater failure to use temporal context information is more difficult to resolve (Parkin \& Hunkin 1993), but it may well prove to be a combined effect of frontal dysfunction (Shimamura et al. 1990) and a loss of recollective information concerning the learning episode.

Until now we have sought to emphasize the distinction between a hippocampal-fornix-anterior thalamic system and a perirhinal-medial dorsal thalamic system. It is, of course, the case that the perirhinal cortex is a major afferent source to the entorhinal cortex and thence to the hippocampus. In view of evidence showing that the perirhinal cortex is important for knowledge concerning objects (Suzuki 1996), principally their familiarity and whether they have been associated with other discrete visual inputs, it can be assumed that this route normally provides object (item)-related information to the hippocampus that may be retained in episodic memory, although other routes can be used following brain pathology (see sect. 4.3). The normal process then involves setting the to-be-remembered item (or items) within its episode or context, and for this association to be possible the hippocampus must receive spatial/ contextual information. In the primate brain the most plausible route is via the parahippocampal cortex (Habib \& Sirugu 1987; Maguirre et al. 1996; Suzuki \& Amaral 1994), which permits item-place representations to be formed. The situation in the rat brain might be different; the postrhinal cortex does not appear to be necessary for some spatial tasks (Aggleton et al. 1997). If, as is assumed, the anterior thalamic nuclei are vital for episodic memory, then these nuclei must interact with those classes of information disrupted by amnesia, including memory for discrete items. Thus, it is to be expected that the pathway connecting the perirhinal cortex-entorhinal cortex-hippocampus-fornixanterior thalamic nuclei forms the route by which discreteitem information is made available for recall.

The assumption that the hippocampus receives objectrelated information from the perirhinal cortex fits with the notion that damage to the hippocampal-anterior thalamic system can disrupt recognition when familiarity information is not available. Further evidence comes from the additive effects of anterior thalamic damage upon posterior medial thalamic damage to DNMS performance by monkeys (Aggleton \& Mishkin 1983a; 1983b). Similarly, fornix lesions on their own have very little effect on the standard DNMS task, but they can exacerbate the effects of cutting the ventral amygdalofugal pathway/temporal stem, which disconnects rhinal projections to the medial thalamus (Bachevalier et al. 1985b). Similar evidence has come from studies of rats showing that fornix damage can accentuate the recognition deficit associated with perirhinal damage (Ennaceur \& Aggleton 1998; Wiig \& Bilkey 1995). In all of these instances, the fornix/anterior thalamic lesion will have involved efferents from the entorhinal cortex as well as the subiculum. The loss of this entorhinal information may contribute to these additive effects.

\section{Final comments}

This target article has been concerned with extending the functional hippocampal system and showing how this relates to the pathology underlying amnesia. What has not been discussed is why this additional diencephalic processing is required. Lesion studies have been relatively unhelpful in this regard because damage to the anterior thalamic nuclei seems to mimic the effects of hippocampectomy so closely. Other approaches are required, and, among these, electrophysiological studies might prove to be especially valuable. An example of this is the work of Gabriel and his colleagues (Gabriel 1993). Using a discriminative avoidance task in which one tone predicts an avoidable shock 
$(\mathrm{S}+)$ and another tone does not predict shock $(\mathrm{S}-)$, training-induced changes in neural activity have been found in both the anterior thalamic nuclei and the posterior cingulate cortex (Gabriel 1993). These consist of increased firing to the conditioned stimuli and greater discriminative firing to $\mathrm{S}+$ than to $\mathrm{S}-$. Because both changes are found in the anterior thalamic nuclei and the upper layers of the posterior cingulate cortex (Gabriel 1993), regions that are closely interconnected, it has been proposed that they reflect an interlinked system. Because this training-induced neuronal plasticity is not observed until late in training, when the association is well learned, it has been described as a "primacy" system, which holds primary or original encodings even after more recent information has been obtained. As might be predicted, lesions of the anterior thalamus-posterior cingulate cortex leave initial acquisition of the avoidance task intact but affect final levels of performance (Gabriel 1993). In contrast, the adjacent medial dorsal nucleus and the interconnected anterior cingulate cortex show discriminative activity from the first session in which behavioural discrimination is observed and are seen as more important in the initial learning stages (a "recency" system).

This model system of avoidance learning offers a means of assessing how the anterior thalamic nuclei can interact with cortical regions in a mnemonic capacity, but the basic avoidance task is unlikely to provide a direct measure of episodic memory and hence is of limited application. Consistent with this is the finding that rabbits with hippocampal lesions can show normal acquisition and performance of the avoidance task (Gabriel 1993), and hippocampal lesions do not disrupt training-induced plasticity in the anterior thalamic nuclei. Conversely, the cingulate cortices are seen as vital for the avoidance task yet are not critical components of the episodic memory system outlined in this target article. There is evidence, however, that the hippocampus modulates overall levels of activity in the anterior thalamic nuclei during the avoidance task. Furthermore, these interactions may be influenced by context, and this may depend on hippocampal activity. Evidence comes from the finding that hippocampal lesions in rabbits attenuate the effects of a context shift on extinction of the conditioned avoidance response (Gabriel 1993). It can be seen that such preparations are moving closer to the demands of an episodic memory system and so may help to address more directly the important issue of why diencephalic relays are required in normal episodic memory.

An underlying assumption in this target article is that the critical contribution of the anterior thalamic nuclei to episodic memory will involve not only its hippocampal and mamillary body inputs but also its other afferents. A similar assumption applies to the mamillary bodies: that is, these structures are contributing something new and not merely passively processing hippocampal outputs. In the case of the anterior thalamic nuclei one potentially important input is the ascending cholinergic projection from the tegmentum. Preliminary evidence comes from the finding that acquiring the discriminative avoidance learning task (Gabriel 1993) leads to increased muscarinic acetylcholine binding in the anterior thalamic nuclei, and this correlates with the appearance of training-induced changes in neural activity (Vogt et al. 1991). A future task will be to look systematically at these and other inputs to the anterior thalamic nuclei and mamillary bodies and thus better determine the nature of the contribution of these structures to the hip- pocampal-anterior thalamic axis. As a consequence, the analysis of lesions will continue to refine our understanding of the critical pathologies underlying aspects of amnesia, but quite different techniques will be required to identify how these different structures contribute to the encoding and maintenance of episodic memory.

\section{ACKNOWLEDGMENTS}

The research of both the authors has been supported by the Medical Research Council (U.K.) and the Wellcome Trust. The authors thank W. Macken and the helpful comments of the referees.

\section{Open Peer Commentary}

Commentary submitted by the qualified professional readership of this journal will be considered for publication in a later issue as Continuing Commentary on this article. Integrative overviews and syntheses are especially encouraged.

\section{Perirhinal cortex: Lost in space?}

\author{
David K. Bilkey \\ Department of Psychology, University of Otago, Dunedin, New Zealand. \\ sycodkb@otago.ac.nz psy.otago.ac.nz:800/academic/bilkey.html
}

Abstract: Aggleton \& Brown argue that the function of the hippocampus and perirhinal cortex can be dissociated along a spatial/nonspatial dimension. They further suggest that this division corresponds to a distinction between episodic and recognition memory. An analysis of the data, however, fails to support the underlying dissociation.

Aggleton \& Brown $(\mathrm{A} \& \mathrm{~B})$ provide a timely reappraisal of the role of temporal and diencephalic structures in amnesia. As part of their reassessment, however, they draw a division between the hippocampus and the rhinal cortex that minimises the influence of the stream of neocortical information that is channeled through perirhinal and entorhinal cortex into this former structure (sect. 4.3). This division is adopted on the basis of evidence from animal lesion studies, where it has been claimed that the function of the perirhinal cortex can be doubly dissociated from that of the hippocampus (fornix/fimbria) on a spatial/nonspatial dimension. Because a major thrust of the $A \& B$ target article is that this spatial/ nonspatial dissociation reflects an episodic/recognition memory dichotomy it is appropriate that we examine this evidence carefully.

Recent experimental findings demonstrate that, whereas lesions of the hippocampus have minimal effects on object recognition memory, lesions of perirhinal cortex produce marked deficits in these processes. These findings, which are summarised in the reviews of Jarrard (1995) and Murray (1996), suggest that this aspect of the dissociation is well founded. The evidence for the reverse aspect of the putative double dissociation, that hippocampal lesions disrupt spatial memory but perirhinal lesions do not, is, however, somewhat more equivocal. In the study of Gaffan (1994a), for example, monkeys were trained in a visually-guided, delayed match-to-sample task, and then received either perirhinal cortex lesions or fornix transections. The animals with perirhinal - but not fornix - damage suffered a decrement in performance on this object memory task. When these animals were subsequently trained in a spatial discrimination and reversal task, however, fornix-, but not perirhinal-lesioned animals were deficient. Although at face value these findings appear to support the spatial/nonspatial dichotomy, it should be noted that these animals 
acquired the visual memory task prior to the lesion procedure, but acquired the spatial task in the post-lesion state. This procedure leaves open the possibility that the "spatial/nonspatial" dissociation may actually reflect the differential effects of the two types of lesion on acquisition versus retention processes. We do not know, for example, whether the perirhinal cortex lesion would have produced a "spatial" deficit if this task had also been acquired preoperatively.

In studies by Ennaceur et al. (1996) and Aggleton et al. (1997) perirhinal cortex lesions did not produce a deficit when rats learned a spatial, delayed nonmatch-to-position task conducted in the T-maze. Although this finding appears to support A\&B's proposal, we have recently shown that excitotoxic lesions of perirhinal cortex will elicit a deficit in this T-maze task, provided that the memory retention interval is increased to beyond one minute (Liu \& Bilkey 1998a). Critically, in the latter study it was demonstrated that animals were unlikely to have used olfactory cues, or a simple left/right response rule, to solve the task. Rather, the most parsimonious explanation of the results was that both lesioned and control animals were solving the task by making reference to a spatial framework, and that lesioned animals displayed faster forgetting of this spatial information. In separate studies we have also demonstrated that lesions of perirhinal cortex disrupt performance in both radial arm maze (Liu \& Bilkey 1998b) and water maze (Liu \& Bilkey 1998c) procedures. Although the former finding differed from that of Ennaceur and Aggleton (1997), raising the possibility that it was caused by damage to fibres of passage, we have recently observed similar deficits in animals with ibotenic acid lesions (Liu \& Bilkey, unpublished data). It is more likely, therefore, that the differences stem from variations in methodology. In the study of Ennaceur and Aggleton (1997), for example, animals received three days of habituation prior to the onset of testing. Each habituation session involved 10 minutes of maze exposure, during which time food was available at the end of each maze arm. Because this procedure is very similar to the actual experimental protocol, it may have allowed time for the lesioned animals to develop a non-mnemonic strategy with which to solve the task prior to the actual testing. It is interesting that when we used a habituation procedure during which food was scattered throughout the maze (thus providing less information about the subsequent test) we observed deficits in the performance of perirhinal cortex-lesioned animals during the first few days of the testing procedure (Liu \& Bilkey 1998b). By the fourth day of the test series, however, we found that lesioned animals began to use a stereotyped, non-mnemonic strategy that appeared to allow their performance to improve to the level of controls.

In summary, recent findings indicate that lesions of the perirhinal cortex produce deficits in tasks that place demands on spatial memory. Further, the results of Liu and Bilkey (1998a) indicate that these deficits are not merely secondary to a deficit in object recognition. Because these findings suggest that spatial information may be processed within several subareas of the temporal region (including both the hippocampus and rhinal cortex), it appears that space/nonspace does not map neatly onto the episodic/ recognition memory dichotomy proposed by A\&B. An alternative approach to the spatial/nonspatial question is to downplay the localisationist aspects of the distinction in preference to a task-oriented approach. This will require an understanding of how problems with a spatial component are decomposed into subtasks, and will involve investigations of the strategies that different brain regions adopt to generate solutions to these problems.

\section{The gap between episodic memory and experiment: Can c-fos expression replace recognition testing?}

\author{
Jan Bures and Andre A. Fenton \\ Institute of Physiology, Academy of Sciences, 14220 Prague, \\ Czech Republic. bures@biomed.cas.cz
}

Abstract: The effort to identify the neural substrate of episodic recall, though ambitious, lacks experimental support. By considering the data on c-fos activation by novel and familiar stimuli in recognition studies, we illustrate how inadequate experimental designs permit alternative interpretations. We stress that interpretation of c-fos expression changes should be supported by adequate recognition tests.

Aggleton \& Brown $(\mathrm{A} \& \mathrm{~B})$ have organized the recent literature on the functions of hippocampal-diencephalic-neocortical circuitry by fractionating recognition into two distinct processes: an effortful elaborative reconstruction of contextual information ("remembering") and a feeling of familiarity-based recognition ("knowing"). It is important that they emphasize that whereas a hippocampal-diencephalic circuit subserves episodic encoding and "remembering," a distinct perirhinal-medial dorsal thalamic circuit underlies "knowing."

Our commentary highlights the lack of experimental support for the neural basis of episodic memory. Clinical and experimental evidence reveals that the perirhinal-medial dorsal thalamic axis is critical for "knowing"; however, without an animal model of episodic memory, evidence for the hippocampal-diencephalic circuit's role in "remembering" is restricted to clinical cases and their inherent limitations. To make their case for the hippocampal-diencephalic circuit, A\&B decompose recognition into "remembering" and "knowing" and then argue that the hippocampal-diencephalic function can be dissociated from the "knowing" process. This does not mean, however, that the hippocampal-diencephalic axis implements "remembering." This can at best be supported by speculating that spatial cells in the hippocampus ("place" and "view" cells), and "head-direction" cells in the anterior thalamus and mammillary bodies could contribute to episodic memory. In fact, directional cells have been found in related areas, including the lateral dorsal thalamus, posterior parietal, and retrosplenial cortices, and lesion studies suggest that the positional and directional systems are independent (reviewed by Muller et al. 1996). The episodic memory hypothesis should be entertained, but one must admit that spatial cells and many properties of the rodent hippocampus have been productively interpreted as a spatial navigation system (O'Keefe \& Nadel 1978). An important merit of A\&B's target article is that it draws attention to the need to characterize the relationship between episodic memory and spatial information processing

Evidence from the rat supporting the role of the perirhinal (and temporal) cortex in "knowing" during the discrimination of novel and familiar objects is based on the increased expression of c-fos in these areas after viewing novel but not familiar stimuli. This weaker c-fos response to familiar objects contradicts the assumption that recognition is an active process that activates the circuits mediating the "familiarity feeling." The c-fos activation elicited by novel stimuli more likely reflects the formation of new engrams rather than the readout of memories representing familiar stimuli. Because the recognizable (i.e., familiar) objects elicit no significant reactions in the perirhinal and TE cortices there is no reason to believe that these regions contribute to "knowing" more than other nonreactive brain structures.

Independent of using relative c-fos inactivity as evidence of "knowing" several experimental issues are critical for interpreting these results.

(1) Using an ingenious within-subject design Zhu et al. (1996) simultaneously exposed one eye to the familiar stimuli and the other eye to the novel stimuli. Because the principle of eye equivalence also applies to the essentially crossed visual pathways of the 
rat, it is probable that the novel objects were recorded in both hemispheres via direct and commissural projections. The absence of c-fos activation in the perirhinal and TE cortices contralateral to the eye seeing the familiar objects, rather than indicating its role in recognition, may indicate either that the callosally transmitted novel stimulus record is too weak or that its formation is prevented by the projection of the familiar stimuli, the processing of which may block the callosal input. These possibilities can be distinguished by covering the eye exposed to familiar stimuli and then examining the effect of familiar or novel objects on the open eye without the masking effect of contralateral input.

(2) Should the two objects simultaneously presented to different eyes be interpreted as a complex scene, the scene would be novel for novel combinations of familiar objects. Zhu et al. (1996) are not explicit about such combinations and allow the reader to assume that the presentation of familiar objects to both eyes is accompanied by (weak) symmetrical c-fos activation. Did the novel combination of familiar objects, contrary to A\&B's model, elicit additional c-fos activation? Wan et al. (1999) distinguish the "object novelty per se" from the "novelty arising from the mismatch of certain learnt associations in space." However, the latter contextual novelty elicited c-fos activity in various hippocampal fields as well as in perirhinal cortex.

(3) A recognition task should be used to study recognition, but this is not done in the c-fos experiments. The rat was exposed to familiar and novel stimuli, but because the reward was independent of the animal's response, it is possible that the rat habituated to the behaviorally irrelevant stimuli. The differential c-fos activation may therefore reflect habituation rather than recognition. A convincing experiment would relate brain changes to behavioral responses showing that the animal can distinguish novel from familiar stimuli (e.g., whereas familiar stimuli require a prolonged nosing response to get additional reward, novel stimuli indicate that no additional reward is available). The same considerations apply to studying episodic memory.

Whereas the perirhinal-medial dorsal thalamic axis is clearly responsible for familiarity-based recognition in primates, the case is not so straightforward in rodents. Similarly, whereas human episodic memory is well characterized, it has proven difficult to study in animals. We hope that Aggleton \& Brown's target article will generate novel experiments clarifying the role of the hippocampal system in the mechanism of episodic memory.

\section{What's new in animal models of amnesia?}

\section{Rebecca D. Burwella and Howard Eichenbaum ${ }^{\mathrm{b}}$ \\ a Walter S. Hunter Laboratory of Psychology, Brown University, Providence, RI 02912; b Laboratory of Cognitive Neurobiology, Boston University, Boston, MA 02215. rebecca_burwell@brown.edu_www.brown.edu hbe@bu.edu www.bu.edu}

\begin{abstract}
In general, we endorse Aggleton \& Brown's thesis that the neuroanatomy of amnesia comprises two functionally distinct systems, but we are disappointed in the lack of detail regarding the critical functional contribution of the hippocampus. We also take issue with the characterization of the cortical areas surrounding the hippocampus, particularly the decreased emphasis on the cortical input to the hippocampus.
\end{abstract}

Aggleton \& Brown (A\&B) present a reformulation of the neuroanatomy of amnesia, one that focuses more on subcortical efferents of the hippocampus and less on its cortical afferents than prior models have done. They further suggest the view that two functional components of amnesia, familiarity judgment and recollection, have different neuroanatomical substrates and can be doubly dissociated. Whereas the reformulation is to be admired for the careful analysis of the neuroanatomy and function of diencephalic contributions to amnesia, we think a few points in their model deserve further consideration.
On the one hand, we enthusiastically endorse the notion that there exist two functionally distinct components of the medial temporal lobe memory system. We are also in agreement with the general distinction between a simpler "recognition" function that is sometimes sufficiently mediated by the cortex surrounding the hippocampus versus a more complex "recollective" function that requires the hippocampus. Indeed, these two points were the central argument of the BBS target article, "Two functional components of the hippocampal memory system" (Eichenbaum et al. 1994). Furthermore, we have no argument with the anatomical extension of the hippocampus component to include the diencephalic connections via the fornix. The idea that subcortical pathways through the fornix are vital for normal hippocampal activity was also a part of that BBS target article, and other earlier models (Mishkin et al. 1984; Papez 1937; Squire et al. 1993).

On the other hand, we were disappointed in A\&B's vagueness regarding the critical functional contribution of the hippocampus, wherein they seem satisfied that "scene memory" and "spatial memory" are equivalent to "episodic memory." They are not, of course, and it would help if they could elaborate on the fundamental cognitive mechanism that relates all these notions. They should include an explanation of why animals with selective hippocampal or fornix damage are so deficient on nonepisodic tasks, for example, learning the water maze (Eichenbaum et al. 1990; Morris et al. 1982) and learning relationships among odors (Bunsey \& Eichenbaum 1996; Dusek \& Eichenbaum 1997).

We also take issue with A\&B's characterization of the cortical areas surrounding the hippocampus. In any formulation of this type, boundaries must be drawn somewhere. A\&B have chosen not to include the cortical regions surrounding the hippocampus in their model of hippocampal-diencephalic contributions to amnesia. Given recent insights into the organization of the pathways by which sensory information is conveyed through the hippocampus, it seems to us a particularly inopportune time to de-emphasize the cortical input to the hippocampus and related brain regions. In the rat and the monkey, the perirhinal and parahippocampal (postrhinal in the rat) cortices provide input to the hippocampal formation via the entorhinal cortex (Burwell \& Amaral 1998b; Naber et al. 1997; Suzuki \& Amaral 1994b). In both species, the perirhinal and parahippocampal cortices project to different subdivisions of the entorhinal cortex. These regions also project directly to the CA1 field of the hippocampus (Kosel et al. 1983; Suzuki \& Amaral 1990). Preliminary observations indicate that, in the rat, the perirhinal and parahippocampal cortices exhibit different patterns of termination in the CA1 (Burwell \& Amaral, unpublished observations). Thus, there are multiple and parallel pathways by which sensory information is conveyed through the hippocampus (Fig. 1). This segregation of input to the hippocampus, both direct and via the entorhinal cortex, is especially interesting because the perirhinal and parahippocampal cortices receive neocortical input from different unimodal and polymodal associational regions (Burwell \& Amaral 1998a; Suzuki \& Amaral 1994a). Taken together, the new anatomical findings suggest that the multiple, parallel pathways by which sensory information is conveyed to the hippocampus may have important functional implications for information processing in the hippocampus and beyond. It seems that this circuitry should not be ignored in a reformulated model of hippocampal function.

The target article does not entirely ignore all cortical input to the hippocampus, only that directed through the entorhinal and postrhinal cortices. A\&B propose that, of the cortical regions surrounding the hippocampus, only damage to the perirhinal cortex contributes to the core deficits observed in amnesia. In constructing their models, $A \& B$ have largely ignored the parahippocampal and entorhinal cortices, as if their interconnections with the perirhinal cortex and hippocampus have no functional relevance. In some sections, the perirhinal cortex and the collected cortical regions surrounding the hippocampus (perirhinal, parahippocampal, and entorhinal cortices) are presented as being functionally interchangeable. This is especially evident as $\mathrm{A} \& \mathrm{~B}$ describe 
Commentary/Aggleton \& Brown: Episodic memory, amnesia, and hippocampus

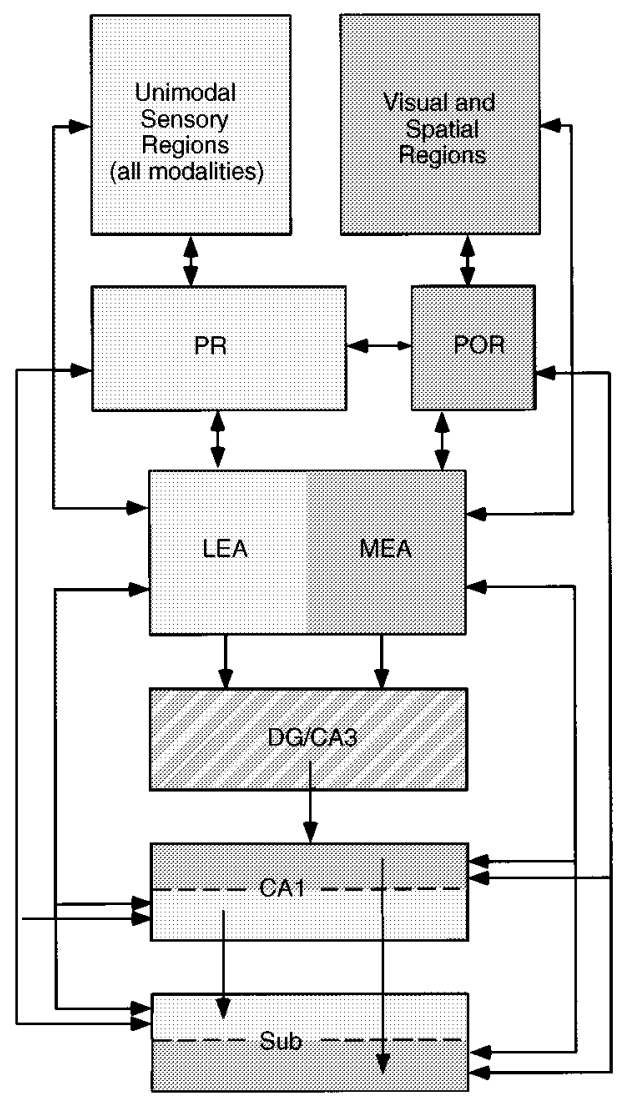

Figure 1 (Burwell \& Eichenbaum). Wiring diagram showing the flow of information through corticohippocampal pathways in the rat. Multiple parallel pathways result in segregation of cortical input to the hippocampus (depicted in light and dark grey). This organization suggests specialization of function between these two sets of parallel pathways. Abbreviations: perirhinal (PR), postrhinal (POR), lateral and medial entorhinal areas (LEA and MEA), dentate gyrus (DG), CA fields of the hippocampus (CA1 and CA3), and the subiculum (sub).

double dissociations between the perirhinal cortex with its subcortical efferents and the hippocampal-diencephalic region. In an earlier work, Eichenbaum et al. (1994) proposed that normal functioning of the hippocampus is dependent on its sensory input arriving from the parahippocampal region. Thus, a double dissociation of the parahippocampal region and the hippocampal region would not be possible. A\&B take issue with this proposal, citing lesion evidence that the perirhinal cortex and hippocampus can be doubly dissociated. We are compelled to point out that experimental lesion studies of the perirhinal cortex versus the hippocampus are not a test of the Eichenbaum et al. (1994) proposal. Because of the convergence of sensory information in the parahippocampal region and the organization of the parallel pathways by which it is conveyed to the hippocampus, substantial sensory information reaches the hippocampus when the perirhinal cortex is damaged (Fig. 1). This input includes somatosensory and olfactory information arriving via the entorhinal cortex, visual and visuospatial information via the postrhinal cortex and the medial entorhinal area, and auditory input arriving via the postrhinal cortex (Burwell \& Amaral 1998a; Suzuki \& Amaral 1994a). Thus, an appropriate test of the Eichenbaum et al. (1994) proposal would compare the effects of hippocampal lesions with those of a combined lesion of the perirhinal, parahippocampal, and entorhinal cortices. The difficulty of making a selective and complete lesion of this large cortical area should not be underestimated, and indeed has never (to our knowledge) been accomplished.

\section{Raising the profile of the anterior thalamus}

\author{
John C. Dalrymple-Alford, Anna M. Gifkins, \\ and Michael A. Christie \\ Department of Psychology, University of Canterbury, Christchurch, New \\ Zealand. j.dalrymple-alford@psyc.canterbury.ac.nz \\ \{psyc002; amg55\}@psych2.psyc.canterbury.ac.nz \\ www.psyc.canterbury.ac.nz/staff/jda/jda.htm
}

\begin{abstract}
Three questions arising from Aggleton \& Brown's target article are addressed. (1) Is there any benefit to considering the effects of partial lesions of the anterior thalamic nuclei (AT)? (2) Do the AT have a separate role in the proposed extended hippocampal system? (3) Should perirhinal cortex function be restricted to familiarity judgements?
\end{abstract}

Aggleton \& Brown's (A\&B's) target article provides a timely perspective on the potential contributions to memory of the limbic diencephalon because the relative influence of the anterior thalamic nuclei (AT) and the dorsomedial thalamic nucleus (DM) have been a major source of conjecture in the recent literature on the neuropsychology of memory. The focus on the AT is perhaps of special importance to the human domain because the human AT, in particular, has more neurones than expected by relative brain size or the number of sensory thalamic and cortical neurones (e.g., Armstrong 1986; Armstrong et al. 1987). As in the recent work by Aggleton and his colleagues (e.g., Steckler et al. 1998b), the idea of an extended hippocampal-AT axis, which mediates the acquisition and normal recall of new episodic information, is a commendable focus on neural networks rather than on isolated brain structures. Hence A\&B's approach provides a welcome addition to our understanding of episodic memory and amnesia. As with any new proposal, however, unanswered questions remain and the evidence that supports the theory has limitations. Three of the issues stimulated by the target article are outlined here.

Contrary to earlier reports (e.g., Beracochea et al. 1989; Greene \& Naranjo 1986), recent evidence from several research groups has now accumulated to show that AT lesions impair performance on tasks that are sensitive to hippocampal system lesions (e.g., Aggleton et al. 1996; Beracochea \& Jaffard 1995; Byatt \& Dalrymple-Alford 1996). We still need to be cautious about this proposed relationship, however, as much remains to be done to test the precise nature of the comparison. One important consequence of this new evidence concerns whether minor damage to the AT, for example within subcomponents such as the anteromedial (AM) and especially the anteroventral (AV) nucleus, is sufficient to disrupt episodic recollection (sect. 5.1, para. 4). Although Aggleton and colleagues imply that such an effect requires substantial damage to the entire AT region, more minor damage can in fact cause clear impairments (Aggleton et al. 1996; Byatt \& Dalrymple-Alford 1996) even when inadvertent AT damage occurs as a consequence of an intended DM lesion that otherwise has no effect on delayed nonmatching-to-position (Hunt \& Aggleton 1991). That the same appears to be less true of mammillary damage serves to highlight the importance of the AT region as a nodal point within the extended hippocampal system (Aggleton \& Sahgal 1993). Conversely, there is little evidence that the laterodorsal nucleus deserves the same status as the conventional parts (i.e., AM; AV) of the AT (see Mizumori et al. 1994; Warburton et al. 1997). Whatever the outcome of the latter issue, we appear to have a situation where relatively minor dysfunction to the AT especially may exacerbate the severity of amnesia caused by an insult elsewhere in the system (for example, with alcoholic or pyrithiamine-induced damage; Beracochea \& Jaffard 1991; Kopelman 1995; Langlais \& Savage 1995). What follows from this is the possibility that disruption to other parts of the hippocampal system can diminish the functional status of the AT region - or vice versa. Thus, an appreciation of the influence of minor damage to the AT leads one to speculate that some recovery of mnemonic function might be produced by manipulating changes to the functional status of either the hip- 
pocampus or the AT, as key regions of an episodic recollection system.

A related issue is whether the AT acts as a simple relay in an extended hippocampal system or has a specific functional role in the context of encoding new information into episodic memory. On the basis of Gabriel's (1993) recordings of training-induced neuronal activity, A\&B speculate that the AT may be involved in late stages of acquisition in perhaps a different manner than that of the hippocampal system (sect. 9). We share their reservations with the shock-avoidance task employed by Gabriel, but the main point is that the hippocampal system may be involved in consolidation processes that extend beyond a possibly more transient role for the AT. The problem for their model is that Gabriel's evidence suggests an earlier, rather than different, role during training for the DM, and functional overlap between an anterior circuit (DM) and a posterior circuit (AT). It may be that under normal (intact brain) circumstances, the AT's influence extends beyond just "episodic memory," but only the latter is dependent on normal AT-hippocampal function.

When recognition is based on familiarity, according to the model, a separate neural system along a perirhinal-DM thalamic axis is proposed as the principal neurological substrate. This knight's move is another welcome break with tradition: The temporal/diencephalic dichotomy is discarded, and we now have a new dichotomy across both diencephalic and temporal lobe structures. Of particular relevance to this idea is the relationship between the hippocampus and the perirhinal cortex, which constitutes a major issue for their model, in which the precise role of the perirhinal cortex seems ambiguous (sect. 4.3). That the mnemonic contributions of the two regions can differ substantially is usually not in question (but see Colombo \& Gross 1996). The problem is that, irrespective of lesion method, the effects of perirhinal lesions may not always neatly fit the suggested distinctions of recollection/ familiarity, spatial/nonspatial (Liu 1998; Liu \& Bilkey 1998b). Instead, lesions here usually produce reliable delay-dependent deficits as their core feature (Eichenbaum et al. 1994); this is not a characteristic of DM lesions even in object-recognition tasks (Hunt \& Aggleton 1991; Parker et al. 1997). In fact, the distinction between a perirhinal axis and an AT axis may be weaker than suggested, as the perirhinal area receives direct AM efferents (Shibata 1993a). An alternative proposal is that perhaps there are multiple, but overlapping, mnemonic systems in which the perirhinal cortex provides a critical temporal lobe interface for both an AT-hippocampal system (encoding for contextual information and relational representations) and a DM-amygdala system (encoding the salience of item-specific information, subserving relative familiarity of both individual items as well as specific emotional stimuli).

\section{That old familiar feeling: On uniquely identifying the role of perirhinal cortex}

\section{J. Eacott \\ Department of Psychology, University of Durham, Durham DH1 3LE, England.m.j.eacott@durham.ac.uk psynt.dur.ac.uk/staff $/ \mathrm{mje} / \mathrm{mje} . \mathrm{htm}$}

\begin{abstract}
Perirhinal cortex contributes to judgements about stimulus familiarity, but its role is far greater than this. Impairments on tasks that do not involve familiarity judgements attest to the fact that perirhinal cortex is involved in the greater role of knowing about objects, including, but not limited to, their relative familiarity.
\end{abstract}

In their summary of the main features of their proposed model, Aggleton \& Brown (A\&B) contrast the recollection process, which depends on the hippocampal-thalamic system, with the detection of stimulus familiarity, dependent on the perirhinal cortex. Much of this idea will be familiar to some (Buckley \& Gaffan 1998a;
Gaffan 1992a; 1994a; Parker \& Gaffan 1998c), although the target article brings together many facets of it in an extremely cogent account of the hippocampal/perirhinal dissociation and the role of the hippocampal-thalamic system. Yet it is less clear what the perirhinal cortex contributes to the memory processes. Under the heading, "Main features of the proposed model," the role of the perirhinal cortex is described as "detecting stimulus familiarity" (sect. 2, para. 4). Later, it is acknowledged that some of the neurons within perirhinal cortex may "encode information concerning the physical characteristics of the stimuli and so may contribute to stimulus identification" (sect. 5.3, para. 4). Yet the role of the perirhinal cortex is repeatedly described as "the detection of the prior occurrence of a stimulus" (sect. 5.3, para. 7) or "familiarity judgements" (Abstract). This view is easy to take on the basis of the electrophysiological evidence that neurons in perirhinal cortex respond most vigorously to novel stimuli (Brown et al. 1987). When it was demonstrated that lesions in this region resulted in deficits in learning or performing delayed match (or nonmatch) to sample (Eacott et al. 1994; Gaffan \& Murray 1992; Horel et al. 1984: Meunier et al. 1993), it became easy to view perirhinal cortex as a region that signalled novel stimuli. However, it is a leap from this evidence to the position that the neurons are simply judging stimulus familiarity.

A neuron that responds more vigorously on first presentation of a stimulus than subsequent presentations could be signalling the novelty of the stimulus, but could equally be doing a number of other things. Although the neurons do respond differentially to novel and familiar stimuli, it is noteworthy that the visual qualities of the stimulus play an equal or greater role in the pattern of the response of these neurons ( $\mathrm{Li}$ et al. 1993). Moreover, whereas the neurons that respond differentially to novel stimuli represent less than a third of the total population of perirhinal neurons in the monkey, the remaining neurons code the physical properties of the stimuli (Fahy et al. 1993b; Li et al. 1993). Thus, the familiarity of the stimulus is not the most important determinant of responding in perirhinal cortex.

Equally, animals with lesions to perirhinal cortex are not always impaired in tasks that require familiarity judgements. Rhinal or perirhinal lesions may result in severe impairments in performance of trial-unique DMS (or DNMS) in monkeys (Eacott et al. 1994; Gaffan \& Murray 1992) and in rats (Mumby \& Pinel 1994; Otto \& Eichenbaum 1992a). However, Eacott et al. (1994) found that DMS performance could be completely unimpaired in monkeys with rhinal lesions if the stimulus set used was very small. The same animals were severely impaired when trial-unique stimuli were used, even when the delay component of the task was removed (Eacott et al. 1994). Thus, these animals were impaired when, and only when, novel stimuli had to be processed, not just when judgements regarding their familiarity had to be made. One interpretation of this finding was that the impairment is one of unique stimulus identification. With trial-unique DMS, the challenge of uniquely encoding stimuli so that they can be discriminated from a great number of potential foils is great and ever present. With the small-set DMS, the same stimuli repeatedly occur so that the identification problem is both less challenging, and, once solved, able to be repeatedly reused.

This interpretation of these data allows an explanation of other behavioural findings that cannot easily be reconciled with the view that perirhinal cortex is merely judging stimulus familiarity. Such an explanation would predict unimpaired performance on tasks that do not rely heavily on judgements of stimulus familiarity, for example, discrimination learning and visual-visual associative learning. Yet reports of impairments in associative learning (Buckley \& Gaffan 1998b; Bunsey \& Eichenbaum 1993; Murray et al. 1993) and discrimination learning (Astur et al. 1995; Buckley \& Gaffan 1997; Eacott 1998; Eacott et al. 1994; Gaffan 1994a; Gaffan \& Murray 1992; Horel et al. 1987; Wiig et al. 1995) following lesions in this region continue to appear. It is important that impairments in discrimination learning are seen where there are a great many discriminanda (Gaffan 1994a), or where they can 
be visually confused (Buckley \& Gaffan 1998c; Horel et al. 1987), thus providing a challenge to identify each one uniquely. Thus perirhinal cortex is not simply signalling that the stimulus has occurred before, but is reflecting knowledge about the object gained in this previous encounter.

An area involved in producing a unique identification of stimuli would be most active when stimuli are presented that have no current representation in the system, that is, when novel stimuli are presented. The activity of such a putative area would reflect the active processing of novel stimuli. Such activity could certainly serve to signal novelty, yet it is not its primary purpose to do so. The presence of a majority of neurons in perirhinal cortex that do not actively engage in processing novel stimuli, but code the defining physical characteristics of a stimulus, might be part of this system. The knowledge about stimuli held by such a system could also include associations with other stimuli (Buckley \& Gaffan 1998b; Bunsey \& Eichenbaum 1993; Murray et al. 1993; Sakai \& Miyashita 1991) and crossmodal associations that bind together different aspects of the same stimulus event to form a full representation of an object.

The idea that perirhinal cortex contributes far more than the simple detection of stimulus familiarity (Buckley \& Gaffan 1998a; Eacott \& Heywood 1995; Murray 1996) fits well within the framework outlined by Aggleton \& Brown's target article. The role of perirhinal cortex is to identify objects as complete and unique entities so that they can be recognised.

\section{Hippocampus, recognition, and recall: A new twist on some old data?}

\section{Jonathan K. Foster \\ Department of Psychology, University of Manchester, Manchester M13 9PL, England.foster@psy.man.ac.uk www.psy.man.ac.uk}

\begin{abstract}
This commentary attempts to reconcile the predictions of Aggleton \& Brown's theoretical framework with previous findings obtained from experimental tests of laboratory animals with selective hippocampal lesions. Adopting a convergent operations approach, the predictions of the model are also related to human neuroimaging data and to other complementary research perspectives (cognitive, computational, psychopharmacological).
\end{abstract}

In their target article, Aggleton \& Brown $(\mathrm{A} \& \mathrm{~B})$ present a timely and clearly articulated framework for explaining the range of episodic memory impairments that can occur after neurological damage. In so doing, they build and elaborate on the views originally expressed by Delay and Brion (1969). They reinforce the emerging viewpoint that the hippocampus is closely involved in mediating relational or associative memory (see Mayes \& Downes 1997).

We can now reevaluate such data thanks to A\&B. More specifically, A\&B provide a valuable opportunity to reappraise some old and hitherto rather puzzling data. Foster and Rawlins (1992a; 1992b) reported two extended experiments in which, contrary to a number of then prevailing views of hippocampal memory functioning (for example, Olton et al. 1979; Rawlins 1985), rats with complete aspiration lesions of the hippocampus were able to perform as well as controls on both unsignalled (Foster \& Rawlins 1992a) and signalled (Foster \& Rawlins 1992b) Go/No-Go tasks. On both tasks, the performance of hippocampal animals was characterised by nonspecific, disinhibited responding (Gray 1982). However, across intertrial intervals ranging from $1 \mathrm{sec}$ to $80 \mathrm{sec}$, hippocampal animals demonstrated unimpaired memory, even when repeated stimuli were used (as in the signalled Go/No-Go task; cf Cassaday \& Rawlins 1995; see sect. 4.1, para. 5 of the target article), and when interference was introduced between trials. At the time, these unexpected sets of findings were interpreted in terms of lesion differences, task discrepancies, the adoption of un- usual response strategies, and/or an interaction between these factors.

On the signalled Go/No-Go task, rats perform efficiently by determining whether the stimulus presented on the present trial is different from the stimulus presented on the previous trial. This can therefore be construed as a test of stimulus recognition. However, on the unsignalled Go/No-Go task, rats ostensibly have to recall whether responding was reinforced on the previous trial (or the trial preceding that, if they are performing optimally and the current trial is a Go trial), to determine whether to respond on the current trial. Therefore, unless one attempts to explain performance on this unsignalled task in terms of familiarity (in which case, one wonders just which subtypes of recall can be mediated via familiarity processes and which cannot), it seems challenging to account for the data from the unsignalled Go/No-Go task within A\&B's framework. (Taken together, these observations also indicate the importance of publishing statistically nonsignificant data, about which journal editors and referees have been known to be somewhat less than enthusiastic!)

In humans, the predictions of A\&B's framework would seem to be consistent with at least some of our findings using structural MRI to evaluate neurobehavioural relationships in the healthy elderly and in patients with Alzheimer's disease. Thus, Foster et al. (1997) noted a significant positive association between hippocampal volume and individual performance on delayed reproduction of verbal and nonverbal materials in a combined sample of healthy elderly and Alzheimer patients. In a second study, conducted by Koehler et al. (1998), in which a larger sample size permitted data from different participant groups to be analysed separately, the original finding with verbal materials was replicated in Alzheimer patients. However, a significant positive relationship was observed in Alzheimer patients in this second study between the volume of the parahippocampal cortex (but not the hippocampus) and delayed visual reproduction (a nonverbal recall rather than a recognition task). This finding appears to be problematic for A\&B. (It is interesting that in the second study, a negative relationship was observed between hippocampal volume and memory performance in the healthy elderly when the data from these individuals was analysed separately. This may be related to the degree of neural pruning that has previously taken place [i.e., during childhood and adolescence] in healthy old brains, although on the surface this finding would also appear to be problematic for at least the simple version of A\&B's framework.)

$A \& B$ 's theory satisfies at least two of the three "desiderata" recently specified when evaluating theoretical models of long-term memory and amnesia (see Mayes \& Downes 1997). Furthermore, the authors marshal an impressive array of data in support of the theory: Their position is constrained by neuroanatomical knowledge, as well as relevant psychological data obtained from testing both patients and laboratory animals. However, the authors are less sure-footed when considering the specific cognitive and computational component processes subserved by particular elements of their proposed anatomical framework.

When considering recognition memory, the authors begin by assuming a trace strength model of recognition. However, I did not find the final paragraph of section 6 very clear. In particular, I was not sure why, in the A\&B model, "more extensive cortical damage" would impair "both processes" (i.e., familiarity and recollection), if recollection is deemed to be subserved by the hippocampus, rather than by cortical regions, and given the evidence cited in section 4.3 that the hippocampus is at least partially independent of anatomical inputs from the perirhinal region. It would also have been helpful if A\&B had made clearer the working definitions they were using when referring in the final paragraph of section 6 both to the "recollection"/"familiarity" distinction (for which they state double dissociations exist) and to the "remember" "know" dichotomy (for which they imply that true double dissociations cannot be demonstrated).

Moreover, $A \& B$ do not appear to be sure about whether they regard "know" responding as a form of implicit memory (see 
sect. 6, end of para. 8, "the loss of 'know' responses in many amnesics ... which contrasts with ... other forms of implicit memory" [my emphasis], whereas in the second sentence of the same paragraph, A\&B state that "familiarity (a feeling of 'knowing') is an essentially explicit, conscious form of memory").

The next paragraph is also not very clear, at least to this reader. $\mathrm{A} \& \mathrm{~B}$ assert that damage to the extended hippocampal system "will result in a loss of associative memory . . . and, hence, source memory," yet other researchers believe that (at least temporal) source judgement is more closely associated with frontal lobe functioning, and indeed we have some unpublished data obtained from testing schizophrenic patients to support this position (Foster et al., in preparation). $A \& B$ do go some way in acknowledging this "frontal" position at the bottom of section 7, paragraph 4 . However (cf sect. 7, para. 1), data obtained in Manchester indicate that the evaluation of trace strength is in fact a poor index for efficient performance of at least some forms of stimulus recency judgement, and that contextual information needs to be encoded efficiently for these temporal judgement tasks to be performed well. The respective roles of the frontal lobes and structures within the medial temporal lobe and diencephalon in mediating recency and other forms of (temporal) source judgement clearly need to be explored further in future research.

$\mathrm{A} \& \mathrm{~B}$ usefully explore the structural and functional connectivity between medial temporal, diencephalic, and prefrontal regions. This approach seems especially worthwhile for those of us who have been somewhat bemused in the past by the use of concepts such as inhibition as explanatory principles in both the hippocampal and frontal lobe literatures (see Fuster 1989; Gray 1982), without (at least regarding the concept of inhibition) much apparent crossover or interaction between these two literatures to date. I would, however, have welcomed some reference to the important role of the prefrontal cortex in memory deficits such as confabulation (which is not an inevitable feature of amnesia), with respect to the postulated role of the prefrontal cortex in retrieval strategies (i.e., initiation and directions of memory search), and/or in the monitoring and verification of search outcome (sect. 2). Indeed, taken as a whole, the focus of the model seems to be on encoding of new episodic information, so that I was left to reflect on how the hippocampal-anterior thalamic system was judged to interact with neocortical regions during reproduction (and consolidation?) of old, fully elaborated memories, and whether in the $\mathrm{A} \& \mathrm{~B}$ model remote memories were thought to be hippocampusdependent or -independent (cf Nadel \& Moscovitch 1997; Squire 1992). (Of note in this context, the frontal lobes have also been linked to the retrieval of remote memories [see Stuss et al. 1994].) It is also not clear to me whether the authors would predict a diencephalic/perirhinal lesion effect that is equivalent in immediate and delayed recognition, or greater for the latter; nor am I clear on the time scale of the role attributed to the hippocampus in mediating recall (in terms of forgetting rate and performance on measures of immediate versus delayed recall).

There is now a developing consensus that different structures within brain circuits, such as the circuit of Papez, may mediate dissociable functional capacities: that is, even when there are predominantly serial connections, individual structures or regions may be involved in separate and independent computational functions. A\&B seem to support this general view (sect. 9: “[T]hese structures are contributing something new and not merely passively processing hippocampal outputs"), although they do not speculate on the statistical algorithms or computational architectures embodied by the anatomical elements cited in their model. These details will need to be fleshed out in future simulations and experimental investigations. In addition, when reflecting on the range of structures implicated by $\mathrm{A} \& \mathrm{~B}$ and other researchers in subserving episodic memory, one is entitled to wonder where to place the structural boundary or outer limit beyond "core memory structures" (as ongoing anatomical projections become more diffuse), when considering the level of interconnectivity between brain regions associated with amnesia.
More generally, I find it puzzling that in the past many researchers either tacitly or explicitly assumed that the anatomical structures within such neural circuits or loops are computationally relatively undifferentiated and apparently passively subserving a common functional or computational goal. This view, which may have represented an inappropriate application of Lashley's principle of equipotentiality, is now fortunately being questioned through the articulation of models such as that put forward by A\&B. Similarly, we now accept that amnesia is not a homogeneous entity, but reflects the family resemblance of a varied group of different memory-related disorders, so that what we should be seeking to establish is a theoretically driven and empirically substantiated taxonomy and detailed explanatory framework of memory disorders and phenomena. In this context, it is notable that in the sections of their target article dealing with human patients, A\&B focus on single case studies to test the validity of their theoretical framework, rather than adopting a syndrome-based approach to "organic amnesia" (see Parkin, 1997, for further examples of the single-case approach to amnesia).

In adopting a convergent operations approach, psychopharmacological and neurochemical approaches, as well as neuroanatomical circuit mapping, must constrain our thinking about memory and amnesia. For example, we have recently demonstrated that the administration of glucose (which may act via the hippocampus) significantly enhances performance on verbal recall but not verbal recognition in healthy young subjects (Foster et al. 1998). This finding would seem to be consistent with A\&B's framework. They acknowledge (sect. 9) that one "potentially important" input to the anterior thalamic nuclei is the ascending cholinergic projection from the tegmentum, although, given that the cited evidence is reliant on correlations, it is not entirely clear whether the authors anticipate that these projections represent critical information-containing inputs or are more nonspecific and modulatory (see Rolls 1996). One way of addressing these sorts of issues in the future would be to implement neuroimaging investigations (such as $\mathrm{fMRI}$ ) in conjunction with precise time-locked EEG measurements.

More generally, anatomically driven theories such as that advanced by $A \& B$ also need to explain the findings from neuroimaging studies of healthy controls, which typically use cognitive challenge to probe the involvement of different brain regions in episodic memory (see Foster, in press). Further developments of the model will therefore need to take into account these complementary perspectives, and in particular, consider the involvement of structures such as the precuneus and the prefrontal cortex, which have been associated with the mediation of long-term episodic memory in several in vivo human neuroactivation studies. By contrast, it has proven more challenging using these neuroimaging techniques to demonstrate activation in the medial temporal and diencephalic brain regions, on which the authors' interest is focused in the target article. Conversely, the evidence adduced by $\mathrm{A} \& \mathrm{~B}$ should demonstrate to computational and connectionist researchers that they cannot attempt to simulate episodic memory effectively through computational modelling of medial temporal lobe structures alone, but that other regions and structures also need to be considered and integrated (see Foster et al. 1997).

Finally, A\&B's caution concerning cross-species comparisons is pertinent (see sect. 1, para. 2), but it also raises the spectre of the extent to which one can legitimately generalise across species, given the central role of language in many instances of human episodic memory. The issue of generalisation is, of course, also central when (language-related issues aside) one is considering the extent to which anatomical homology of brain regions maps onto functional analogy across different species (e.g., from rats to humans). 


\section{What does the limbic memory circuit actually do?}

\section{Michael Gabriela and David M. Smith ${ }^{\mathrm{b}}$}

a Department of Psychology and Beckman Institute, University of Illinois, Urbana, IL 61801; b Neuroscience Program and Beckman Institute, University of Illinois, Urbana, IL 61801. \{mgabriel; dmsmith\}@uiuc.edu

Abstract: We applaud Aggleton \& Brown's affirmation of limbic diencephalic-hippocampal interaction as a key memory substrate. However, we do not agree with a thesis of diencephalic-hippocampal strict dedication to episodic memory. Instead, this circuitry supports the production of context-specific patterns of activation that subserve retrieval for a broad class of memory phenomena, including goal-directed instrumental behavior of animals and episodic memory of humans.

Support marshaled by Aggleton \& Brown (A\&B) for the essential involvement of hippocampal-diencephalic (H-D) interactions in recollection (but not recognition) has potential to confer on the limbic diencephalon its long-deserved (and widely ignored) status as a legitimate participant in the limbic memory circuit. Yet, we find problematic the main thesis: H-D dedication to episodic memory. We argue instead that the H-D interactions subserve a broader mnemonic function, cue-, and context-encoding in the service of retrieval. This function is used in multiple memory paradigms including basic instrumental learning of goal-directed behavior of animals and episodic memory.

Our studies of the neurophysiological bases of discriminative instrumental learning in rabbits (cited in the target article; Gabriel 1993) indicate an essential involvement of the limbic diencephalon, and a clear relevance of H-D interactions to this learning. The results are in accord with A\&B's general thesis that the $\mathrm{H}-\mathrm{D}$ axis is involved in memory-relevant processing. We appreciate their acknowledgment of our work (sect. 9, "Final comments"). Yet, A\&B were ambivalent concerning the relevance of our data to their account, as indicated by their comment that "the basic avoidance task is unlikely to provide a direct measure of episodic memory and hence is of limited application." This arises from the authors' conviction that the hippocampus, clearly important in mediation of episodic memory, is not involved in basic instrumental learning. Admittedly, the hippocampus is not essential for acquisition of discriminative avoidance behavior. However, it modulates the behavior as well as the task-relevant neuronal activity of the anterior thalamus, as indicated below.

Extensive recordings of neuronal activity during behavioral acquisition demonstrate unique topographic distributions of brief latency, S+elicited neuronal activity across distinct nuclei of the anterior thalamus and the layers of posterior cingulate cortex. Certain thalamic nuclei and cortical layers were maximally activated by the $\mathrm{S}+$ in the initial session of training, others in intermediate training stages, and still others as the rabbits attained asymptotic discriminative performance (Gabriel et al. 1991). The distributions of activation changed systematically, not only across time (training stage) but also with respect to the spatial context. The same physical cues elicited different patterns of activation, depending on whether the subjects were engaged in a moderatelylearned discriminative avoidance task, or (in a separate training apparatus) a well-learned discriminative approach task (Freeman et al. 1996). Thus, the distribution patterns coded both the spatial and temporal context. These context-specific patterns, elicited at brief latency $(80 \mathrm{msec})$ by the cue $(\mathrm{S}+)$ that calls forth the learned response, are arguably the brain's earliest sign (in the millisecond series) of context-specific retrieval.

Other evidence suggests that the context-specific patterns depend on the integrity of hippocampal efferent flow to the limbic diencephalon and cingulate cortex. Subicular, hippocampal, and entorhinal cortical lesions alter anterior thalamic and cingulate cortical task-related neuronal activity in ways that are likely to degrade the value of the patterns as a spatio-temporal context code (Freeman et al. 1997; Gabriel et al. 1987; Kang \& Gabriel 1998). Moreoever, the lesions impair contextual processing. Rabbits with lesions failed to reduce avoidance responding when the familiar training context was altered (reviewed by Gabriel 1993; see also Freeman et al. 1997). This failure of context-specificity was not simply a "response inhibition" problem as the rabbits inhibited normally when $\mathrm{S}+$ quality was altered instead of the context (Gabriel 1993, p. 515).

These and many other findings implicate the hippocampus and the anterior thalamus in mediation of context-specific retrieval of discriminative, instrumental, goal-directed behavior of rabbits. We propose further that these areas are involved in the retrieval of episodic memory, a point on which we and the authors are in agreement. However, our account does not place responsibility for the whole of episodic memory on the hippocampal-diencephalic circuitry. The circuitry does not, in our view, store items or contents of memory as A\&B seem to suppose (sect. 7). Rather, associative synaptic modifications are stored, which allow familiar and significant stimuli to elicit context-specific patterns of activation. The patterns, in turn, retrieve context-appropriate contents of memory. The contents of memory, be they goal-directed behaviors or experiential recollections (episodic memories), are stored elsewhere in the brain (in different functional circuitries) and are recalled by virtue of their association with the limbic retrieval patterns. Thus, the more general issue concerns the appropriate mapping of components of a complex construct (e.g., episodic memory) onto the brain's distinct functional circuits.

Our view integrates many findings in addition to the foregoing. For example, the limbic diencephalon's mediation of discriminative avoidance behavior is time-limited. Retention of the behavior was severely impaired when anterior and MD thalamic lesions were made after training to criterion, but no impairment was found when the lesions were made after 10 days of post-criterial overtraining (Hart et al. 1997). Just as for episodic and declarative memory, discriminative avoidance learning undergoes gradual "consolidation," that is, a progressively acquired independence from limbic circuit processes. (Given the strong consensus that episodic memory is subject to consolidation, we were surprised that A\&B did not explicitly address this issue.)

The fact that hippocampal lesions do not impair acquisition of discriminative behavior (whereas they do impair episodic memory) is not a compelling justification for regarding these forms of learning as products of entirely separate memory systems. Discriminative avoidance behavior is acquired normally by subjects with damaged hippocampi because original acquisition poses little challenge to the limbic retrieval circuit. Retrieval during acquisition does not require or use the exquisite thalamic spatiotemporal patterns endowed by hippocampal efferents for disambiguating inputs or for response selection. It occurs instead as a result of direct information flow from the limbic thalamus and cingulate cortex to areas involved in priming and execution of the locomotor response.

The absence of a hippocampus is noticed when there is potential for substantial mnemonic interference, such as when multiple, similar tasks are learned concurrently, when retrieval cues are similar to cues associated with already-stored items, or when subjects are required to use different configurations of contextual stimuli for selection among response alternatives. It is therefore not surprising that a hippocampal contribution to instrumental performance is detected when transfer-of-training tests are given, such as testing in a novel context, training with cue-reversal, or requiring subjects to base their behavior on complex spatial configurations. Substantial intereference is commonplace, however, in studies of episodic memory, which typically involve complex stimuli and multiple response items. 


\section{Episodic memory in semantic dementia: Implications for the roles played by the perirhinal and hippocampal memory systems in new learning}

\section{Kim S. Graham and John R. Hodges \\ MRC Cognition and Brain Sciences Unit, Cambridge, CB2 2EF, England. \{kim.graham; john.hodges\}@mrc-cbu.cam.ac.uk www.mrc-cbu.cam.ac.uk}

Abstract: Aggleton \& Brown (A\&B) propose that the hippocampal-anterior thalamic and perirhinal-medial dorsal thalamic systems play independent roles in episodic memory, with the hippocampus supporting recollection-based memory and the perirhinal cortex, recognition memory. In this commentary we discuss whether there is experimental support for the A\&B model from studies of long-term memory in semantic dementia.

One of the central premises underlying Aggleton \& Brown's (A\&B's) reformulation of the neuroanatomy of episodic memory is that the hippocampal-anterior thalamic and perirhinal-medial dorsal thalamic systems have dissociable functions: whereas the former is critical for the efficient recall of true episodic information, the latter supports recognition memory based primarily on judgement of familiarity. This provocative reformulation predicts that patients with damage to the hippocampal-anterior thalamic versus the perirhinal-medial dorsal thalamic system will show a double dissociation on tests of recognition- and recall-based episodic memory. In support of this hypothesis, A\&B draw attention to the three patients described by Vargha-Khadem and colleagues (1997) who suffered isolated bilateral hippocampal damage early in life: All three patients showed disproportionately better recognition memory, despite poor spatial, temporal, and episodic memory. In support of the opposite neuropsychological pattern, A\&B cite the patients described by Kapur et al. (1992; 1994) who showed impaired performance on the faces version of the Recognition Memory Test (Warrington 1984), but normal recall on tests of visual nonverbal memory.

$A \& B$ also suggest that data from patients with the neurodegenerative disorder semantic dementia (Graham \& Hodges 1997; Hodges \& Patterson 1996; Hodges et al. 1992; Snowden et al. 1989) are relevant to their model. Patients with semantic dementia provide memory researchers with a unique opportunity to investigate the organisation of episodic and semantic memory in the human brain because the neuroanatomical damage is complementary to that typically seen in patients with amnesia: focal atrophy to the inferior and middle temporal gyri with preservation of medial temporal lobe structures, including the hippocampus (Harasty et al. 1996; Mummery et al., in press).

Although $\mathrm{A} \& \mathrm{~B}$ describe the reverse retrograde amnesia reported in semantic dementia (see below), they do not discuss the potentially more relevant findings from studies of new learning in semantic dementia (Diesfeldt 1992; Graham et al. 1997; Warrington 1975). For example, recent studies from our research group have demonstrated spared recognition memory at early stages in the disorder: Graham et al. (1997) showed normal two-alternative, forced-choice recognition memory using a real/nonreal object decision task in a group of five patients with semantic dementia compared to control subjects. A comparison group of amnesic patients in the early stages of Alzheimer's disease showed impaired recognition memory on the same test. A further study in a group of eight patients with semantic dementia has replicated this finding (Graham et al., submitted): A double dissociation between performance on a three-alternative, forced-choice recognition memory test was found in patients with semantic dementia (preserved memory) and early Alzheimer's disease (impaired memory). Deficient recognition memory was only demonstrable in semantic dementia if "unknown" targets seen at study (i.e., stimuli that were no longer familiar to the patient as measured by picture naming and/or word-picture matching) were replaced by a different example of the same item in the recognition memory task (i.e., changing a round-dial telephone seen at study with a touch-button phone at test). We interpreted this result as evidence for multiple inputs to medial temporal lobe structures, and suggested that higher-order perceptual information is sufficient to support recognition memory in semantic dementia, even when the item to be remembered is no longer familiar to the patient.

The relevance of the neuropsychological data from semantic dementia to A\&B's model hinges on whether there is pathological involvement of the perirhinal cortex in these patients. If patients do possess damage to this area of the temporal lobe, the preservation of recognition memory in semantic dementia would be contrary to much of the animal literature in which perirhinal damage results in impaired recognition memory, even for perceptually identical stimuli. On the other hand, if the perirhinal cortex is relatively unaffected in the disease, the preserved recognition memory seen in such patients would concur with A\&B's model. Clearly there is a need for accurate quantitative structural neuroimaging in semantic dementia to demonstrate preservation or damage to perirhinal, entorhinal, and hippocampal areas. The exact location of the perirhinal cortex in man remains somewhat controversial, however. Current neuroanatomical studies indicate that whereas the rostral portion occupies the banks of the collateral sulcus, the caudal part extends onto the medial temporal pole (Corkin et al. 1997; Van Hoesen 1997). Clinical MRI studies in semantic dementia suggest consistent atrophy of the temporal pole but relative sparing of the parahippocampal gyrus and collateral sulcus. Preliminary findings from a voxel-by-voxel structural analysis of regions of significant difference in grey matter density in six patients with semantic dementia, compared to a control group, also indicate that the pathology may not extend into either the perirhinal or entorhinal cortices (Mummery, unpublished data).

There is reason to believe, therefore, that further neuropsychological studies of new learning in semantic dementia, combined with detailed structural analyses of individual areas within the patients' temporal lobes, will provide additional support for A\&B's model. A further question remains: How does the data on new learning in semantic dementia equate with A\&B's distinction between familiarity- and recollection-based episodic memory? The integrity of recognition memory, considered in conjunction with the preservation of recent autobiographical memories, suggests that both types of episodic memory may be preserved early in the disorder. Of course, we are not suggesting that new learning remains normal in semantic dementia throughout the course of the disease. In fact, three of the patients described in Graham et al. (submitted) showed a significant, although mild, impairment on recognition memory for perceptually identical stimuli (see also Graham et al. 1997). On the basis of A\&B's proposal about the role of the perirhinal cortex in recognition memory, there may be a clear stage in the disease when perirhinal-dependent recognition memory may be disproportionately more affected than the recollection of temporal and spatial information (as demonstrated in Kapur et al.'s [1992; 1994] patients).

Turning now to the issue of retrograde amnesia: As cited by A\&B, experiments have demonstrated that patients with semantic dementia show a reverse Ribot gradient, whereby there is better retrieval of recent memories compared to those from the more distant past, on tests of autobiographical memory and knowledge of famous personalities and public events (Graham \& Hodges 1997; Graham et al., in press; Hodges \& Graham 1998; Snowden et al. 1996). These studies highlight the time-limited role played by the hippocampal complex in the encoding and storage of autobiographical and semantic memories: Typically, patients with semantic dementia are able to produce autobiographical memories and semantic knowledge only for the last two to three years of their lives (Graham \& Hodges 1997; Hodges \& Graham 1998). Although A\&B have undertaken a comprehensive review of anterograde amnesia, it is not clear from their target article how their reformulated model corresponds to the double dissociations seen on tests of remote memory in patients with selective damage to hippocampal or neocortical areas of the brain. More specifically, how do the different 
memory systems (perirhinal versus hippocampal) contribute to learning of semantic and episodic memories? We presume that $\mathrm{A} \& \mathrm{~B}$ would adopt a position similar to that of Vargha-Khadem and colleagues (1997), who suggested that the perirhinal and entorhinal cortices can support context-free, long-term semantic learning (as seen in their three developmental cases), but that the hippocampal system is critical for normal acquisition and storage of contextdependent autobiographical memories.

In summary, despite our current lack of understanding concerning the next neuroanatomical pattern of damage in semantic dementia, and the neuropathological evolution of the disease over time, it seems that there is preliminary support from semantic dementia for Aggleton \& Brown's model. Furthermore, there are clear theoretical predications regarding the integrity of recognition and recollection memory over the course of the disease that could be addressed in further experimental studies. The authors are to be congratulated on a clear and compelling review of the sometimes confusing and contradictory literature on anterograde amnesia in humans and animals.

\section{Retrieval dynamics and brain mechanisms}

\section{Douglas L. Hintzman}

Department of Psychology, University of Oregon, Eugene, OR 97403. hintzman@oregon.uoregon.edu

Abstract: Experiments on memory-retrieval dynamics support the hypothesis that different mechanisms mediate processes of familiarity and recall (recollection). The minimal retrieval time for accurate recognition and frequency judgments is about 100 msec earlier than the minimal time to judge details such as modality or location. The difference is consistent with neurophysiological evidence. Retrieval dynamics data are also relevant to other aspects of Aggleton \& Brown's target article.
Aggleton \& Brown's (A\&B's) proposal that different brain circuits mediate judgments of familiarity on the one hand, and the recall or recollection of experiential details on the other, may help explain certain experimental findings on the dynamics of memory retrieval in normal humans. In the response-signal (or speed-accuracy tradeoff) method, the experimental subject is shown a retrieval cue such as a word, and after a variable lag is given a signal demanding an immediate binary (yes-no) response. The response might indicate whether the cue is old or new, or reflect some other decision about the cue that is based on memory. If the signal to respond comes at a very short lag after cue onset, decision accuracy is at chance (hit and false alarm rates are the same). If the signal comes after a long lag, accuracy is at asymptote. In between, accuracy typically traces out a curve that rises from chance abruptly, and can be closely fit by an exponential function. The point at which the function first starts to rise above chance (the intercept) is an estimate of the minimal time required to retrieve and evaluate relevant information and execute a response.

Several experiments have compared the response-signal functions from different memory-judgment tasks, holding subjects and materials constant. In Experiment 2 of Hintzman et al. (1998), for example, subjects saw two successive word lists. Some words appeared once and some twice in the same list. Next, subjects were told whether the response-signal tests to follow would require old versus new decisions (recognition) or list 1 versus list 2 decisions (list discrimination). Figure 1 shows discrimination performance in the two tasks, as a function of processing time (lag plus response latency). Whereas asymptotic performance was affected by both the task and the number of repetitions, the intercept was determined primarily by the task. Specifically, for about $100 \mathrm{msec}$ early in the retrieval episode, information was available that could be used in making accurate recognition decisions, but not in judging whether the test word came from list 1 or list 2.

The response-signal method has been used to compare other

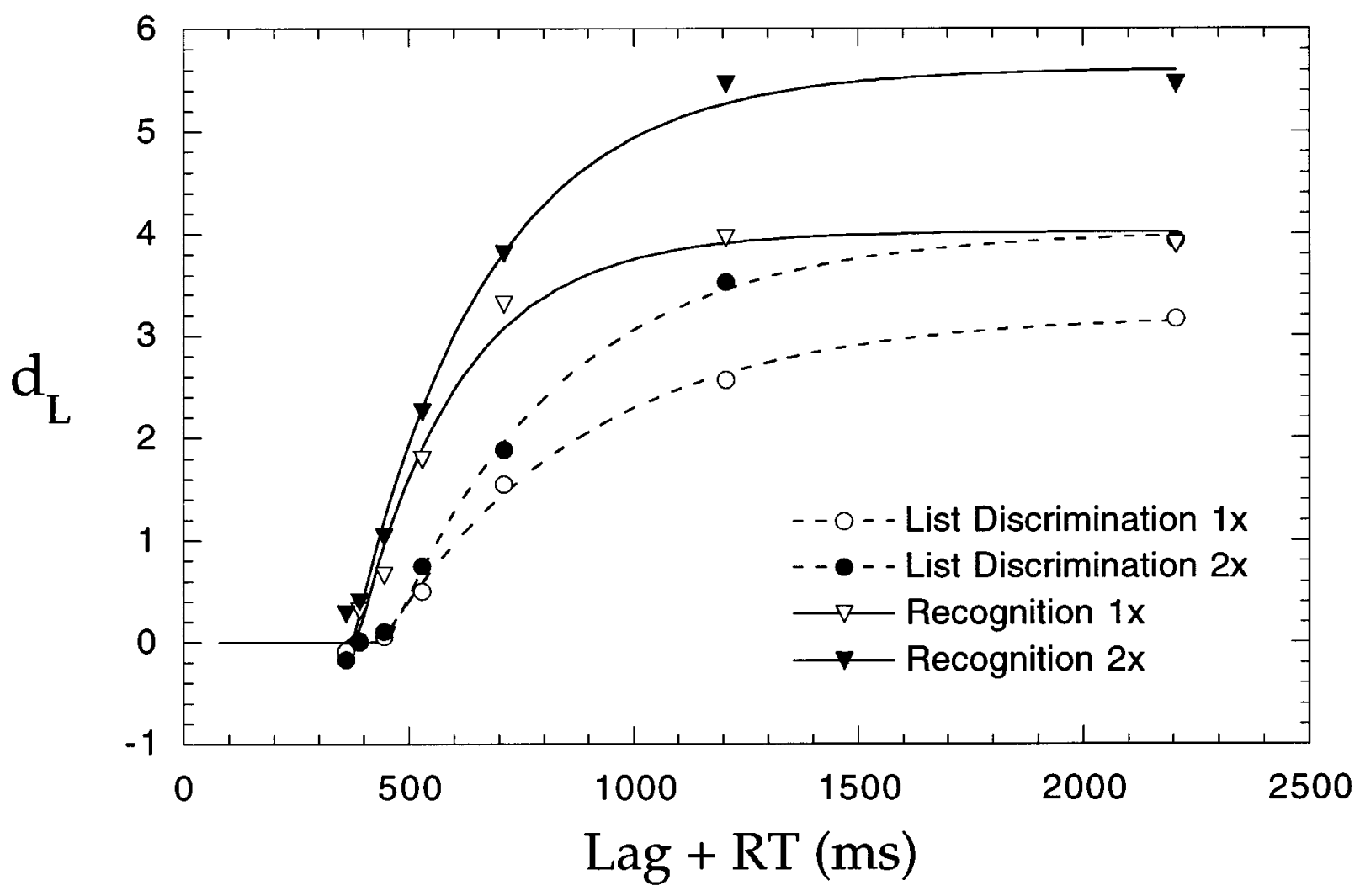

Figure 1 (Hintzman). List-discrimination and recognition-memory performance as a function of lag plus mean reaction time (Hintzman et al. 1998); $\mathrm{d}_{\mathrm{L}}=$ accuracy; $1 \mathrm{x}=1$ study trial; $2 \mathrm{x}=2$ study trials. 
discrimination tasks with recognition memory, with similar results. Recognition intercepts are shorter for recognition than for judging the auditory versus visual study modality of a word (Hintzman \& Caulton 1997), for judging whether the test word is in the same spatial location in which it was studied (Gronlund et al. 1997), and for detecting whether the word's plurality (FROG vs. FROGS) has changed on the test (Hintzman \& Curran 1994, Exp. 3). By contrast, frequency judgments may be based on the same information as recognition. In Hintzman and Curran (1994) Experiment 1, the intercept for judging whether a test word had been seen one time or two times was as early as that for judging whether a test word was old or new. With the exception of frequency judgments, each of these memory-judgment tasks has an intercept significantly longer than that of recognition memory. The size of the obtained difference is usually around $100 \mathrm{msec}$.

The early and late intercepts may reflect minimal retrieval times for the two memory circuits proposed by A\&B (see also Eichenbaum et al. 1994). The perirhinal-thalamic circuit supports retrieval of familiarity, and could mediate judgments of both recognition and frequency. The hippocampal-thalamic circuit supports recall of the kinds of contextual and structural information that would be needed to make judgments of list membership, modality, spatial location, and plurality. Retrieval from the hippocampal system might be expected to take a longer time, because it is computationally and anatomically more complex. Studies using monkeys indicate that a familiarity decrement in neural responding first shows up at 70-80 msec after stimulus onset in inferotemporal cortex (Miller et al. 1993), and at 140-260 msec in hippocampus (Rolls et al. 1993). Comparisons across species (and across labs) can be problematic, but the difference between these figures is intriguingly similar to that between the response-signal intercepts that I have described.

Incidentally, response-signal results also suggest that the familiarity that underlies recognition judgments should not be identified too closely with perceptual fluency. For purposes of timing control, response-signal test cues are presented visually. When some test words were studied auditorily and some were studied visually - exactly matching the test cue - no difference was found in their recognition intercepts. Although this is a null result, it has been replicated at least three times (Hintzman \& Caulton 1997; Mulligan \& Hirshman 1995). It suggests that perceptual fluency caused by exact stimulus repetition is not an important factor in recognition.

Enhanced fluency does show up, however, in the response-signal intercept for lexical decisions - particularly in the case of low-frequency words (Hintzman \& Curran 1997). The lexical-decision intercepts for low-frequency words that were primed by repetition, and for primed and unprimed high-frequency words, are significantly earlier than that for recognition. The recognition intercept seems to be unaffected by study modality, repetition, or frequency of the word in the language, all of which affect priming or fluency. These retrieval differences between recognition and lexical decision suggests there is more than one kind of familiarity, a conclusion that is also consistent with the literature reviewed by $\mathrm{A} \& \mathrm{~B}$.

\section{Neuropsychological assumptions and implications}

\section{Narinder Kapur}

Wessex Neurological Centre, Southampton General Hospital, Southampton, England S016 6YD. n.kapur@soton.ac.uk

\begin{abstract}
Some of the assumptions underlying the arguments in Aggleton $\&$ Brown's target article are reviewed; discrepancies/predictions are pointed out in relation to human lesion studies. A\&B's proposal is interesting, but it may require harder, confirmatory evidence before it can be considered to be all-encompassing.
\end{abstract}

Aggleton \& Brown's (A\&B’s) is an interesting and stimulating target article. However, the authors seldom make it clear whether and to what extent they are relying on human neurobiological findings as bases for their arguments. For example, in the case of neuroanatomy, Crick and Jones (1993) have powerfully pointed to our ignorance in this field. In addition to species differences in human morphology, there may also be possible differences in blood flow, neurochemistry, anatomical connectivity, inhibitory-excitatory harmony, and so forth. In an article such as A\&B's there is inevitably a jumping from one species-platform of data to another, and it is important to note the assumptions that go along with such conceptual leaps.

One of our problems in amnesia research is that we do not know, or at least have not agreed on, what we mean by amnesia. That is, there is no agreed set of criteria by which we can classify a patient as amnesic, or - more importantly - give an indication of the severity of amnesia. This applies both to anterograde and retrograde amnesia. A further issue is of course the purity of amnesia. This is also important, though not as difficult a problem to resolve. To take one or two examples from the types of cases reviewed by A\&B: the patient RB (Zola-Morgan et al. 1986) and our own patient BJ (Kapur et al. 1994) were both moderately amnesic by most standards, and our own patient has in fact gradually improved over the years so that he might now be classified as "mildly amnesic." Can one build the same theoretical arguments around these patients, and include them in the same database as patients such as HM (Corkin 1984) or CW (Wilson et al. 1995) who are by all accounts among the most severely amnesic patients ever seen?

In the case of the mammillary bodies, it is - as $A \& B$ point out - very difficult to find cases of discrete, isolated damage to these structures in the human domain. We have recently come across two cases of suprasellar tumours (Kapur et al. 1999) where there appeared to be minimal additional damage apart from that to the mammillary bodies. In both cases, the memory loss was moderate rather than severe.

Similarly, there are few human cases of discrete, isolated lesions of the rhinal cortex. There is one paper, not cited by A\&B (Yoneda et al. 1994), which pointed to a role for the rhinal cortex in retrograde but not anterograde memory. In the case of the thalamic component of their argument, the authors should note and incorporate negative cases of thalamic damage but normal memory, such as that of Kritchevsky et al. (1987).

I would have liked to see a few comments about whether in the limbic-diencephalic memory system proposed by $A \& B$ we are dealing with an "analogue" system or a "digital" system. That is, is it that the greater the number of components in the system that are damaged, the more severe the amnesia, or is there a threshold point after which amnesia suddenly develops and remains at that level of severity regardless of additional damage? Similarly, is the pattern of memory loss (e.g., recall versus recognition) the same after each component is damaged, or do different components of the limbic-diencephalic system have a unique, distinctive contribution to make? The answers may be present in the target article, but they do not come across as clearly as one would have liked.

If $\mathrm{A} \& \mathrm{~B}$ are arguing that the perirhinal-medial dorsal thalamic system selectively subserves familiarity recognition memory judgements, with little role in recall, then would one predict human cases with damage to this system to show impaired recognition memory (or perhaps impaired priming) but intact recall memory? In the few studies purporting to show such an unusual pattern of memory loss (e.g., Delbecq-Derousne et al. 1990), the pathology has mainly been in or near the frontal lobes.

The single-cell recording studies reviewed by A\&B provide fascinating clues as to the bases of memory processes such as familiarity recognition memory, but it is important to remember that without simultaneous multiple recordings elsewhere in the brain it is not possible to be certain about the significance of these specific sets of recording data, in particular, the stage of any sets of neuronal firing within an overall network of related cell assemblies. 


\section{Perirhinal cortex and hippocampus mediate parallel processing of object and spatial location information}

\section{Raymond P. Kesner \\ Department of Psychology, University of Utah, Salt Lake City, UT 84112. rpkesner@behsci.utah.edu www.utah.edu}

\begin{abstract}
An alternative to Aggleton \& Brown's interpretation is presented suggesting that the perirhinal cortex and hippocampus mediate different attribute information, but use the same processes, supporting the idea of parallel processing based on attribute (visual object and spatial location) rather than process characteristics (item recognition and familiarity).
\end{abstract}

Aggleton \& Brown (A\&B) have provided a scholarly review, including relevant data from both the animal and human literature to support the idea that the perirhinal cortex and the hippocampus mediate two different systems labeled as item recognition and familiarity. The distinction is based on assumed differences in processing of information. An alternative interpretation of the data is that the differences between the hippocampus and perirhinal cortex reflect the involvement of the hippocampus in processing spatial attribute information and reflect the involvement of the perirhinal cortex in processing of visual object information. To support the aforementioned alternative, one needs to question whether it is mandatory to apply the concept of item recognition only to visual object information and not to spatial location information. The concept of item recognition has been more readily applied to visual object information, because one can identify the critical stimulus to be remembered more easily than is the case for spatial locations, which are often assumed to be based on relational representations. I would suggest, however, that both visual object and spatial location information are represented by an integration and relational representation of many features and thus one should be able to apply the concept of item recognition to spatial locations as well as to visual objects. Even Aggleton has stated in a different article that spatial location recognition memory can be as important as visual object recognition memory (Steckler et al. 1998a). Also, familiarity judgments are not likely to depend on one specific circuit; rather, they may represent a process that subserves different attributes of memory. To support this idea I have used a continuous recognition memory paradigm using either spatial locations or visual objects. This is an ideal paradigm in that one could easily point to the operation of familiarity as well as item recognition.

For the visual object version, the continuous recognition memory task involves sequential presentation of 12 three-dimensional objects within a session selected from a set of 120 objects. From the 12 objects, 8 were novel and 4 of the 8 were presented twice. Repeated objects have lags ranging from 0 to 4 (from 0 to 4 different objects are presented between the first and the repeated presentation). Rats are reinforced for approaching the novel object, but they are not reinforced for a repetition. Rats learn this task quickly and show significantly longer latencies to approach and move the repeated compared to the novel objects (familiarity). Furthermore, the shorter the lag the greater the latency difference.

For the spatial version, the continuous recognition memory task involves sequential presentation of 12 maze arms within a session. Of the 12 presentations, 3 or 4 of the arms are repeated. Rats are reinforced for approaching the new arm for that session, but they are not reinforced for a repetition. Repeated arms are presented with lags ranging from 0 to 6 (from 0 to 6 different arms are presented between the first and the repeated presentation). Rats learn this task quickly and show significantly longer latencies to approach the repeated arm compared to the nonrepeated arm (familiarity). Furthermore, the shorter the lag the greater the latency difference.

It is important to point out that these two tasks are analogous, so that comparisons can be more readily made concerning the op- eration of familiarity and item recognition. The results indicate that perirhinal cortex lesions disrupt visual object-based continuous recognition memory across all lags, and that hippocampal lesions disrupt spatial location continuous recognition memory across all lags, but hippocampal lesions do not impair visual object continuous recognition memory across any lags ( Jackson-Smith et al. 1993; Kesner et al., submitted). Because the deficits appear for all lags, the data suggest that the perirhinal cortex supports shortterm visual object recognition memory as well as visual object familiarity and that the hippocampus supports short-term spatial location recognition memory as well as spatial location familiarity. Thus, this alternative interpretation is based on the assumption that there are separate neural circuits for memory for spatial location (hippocampus and interconnected neural circuits) and memory for visual objects (perirhinal cortex and interconnected neural circuits). Yet these two neural circuits are part of the same memory system, which has been labeled as data-based memory or declarative memory.

Further support for this alternative interpretation is based on the assumption that specific operational characteristics are associated with each attribute as part of the data-based memory system. These include a number of processes, such as pattern separation based on selective filtering or attenuation of interference associated with temporary memory representations of new information, short-term memory or working memory of new information, short-term memory or working memory of new information, consolidation or elaborative rehearsal of new pattern association information, and retrieval or pattern completion of new information based on flexibility and action. It can be shown that the hippocampus mediates pattern separation, short-term memory, consolidation, and retrieval of new spatial information, whereas the perirhinal cortex can be shown to mediate pattern separation, short-term memory, consolidation, and retrieval of new visual object information (for a review see Kesner 1998). Thus, the two neural regions (perirhinal cortex and hippocampus) mediate different attribute information, but use the same processes, supporting the idea of parallel processing based on attribute rather than process characteristics.

\section{Recall, recognition, and the medial temporal lobes}

\section{Barbara J. Knowlton \\ Department of Psychology, University of California, Los Angeles, Los Angeles, CA 90089. knowlton@psych.ucla.edu}

Abstract: Aggleton \& Brown predict that recognition and episodic recall depend on different brain systems and can thus be dissociated from one another. However, intact recall with impaired recognition has not yet been demonstrated if the same type of information is used in both tests. Current evidence suggests that processes underlying familiarity-based recognition are redundant with processes underlying episodic memory.

Aggleton \& Brown (A\&B) put forth an intriguing view that places medial temporal lobe structures subserving declarative memory in their anatomical context. Their view makes a very straightforward prediction, that recall and recognition are independent and can therefore be doubly dissociated from one another in neuropsychological patients. Although it is clear that there are patients who exhibit deficits in recall in the face of normal recognition, it is not yet clear that there are patients who can consistently recall items despite an inability to recognize them.

It is well known that patients with prefrontal damage exhibit deficits in free recall despite good recognition abilities (see Shimamura 1995 for a review). These patients appear to have difficulty with the strategic aspects of recall - effectively generating cues and using them to build a memory. This deficit seems especially pronounced for episodic memories, which by definition are 
tied to a particular place and time. The deficit frontal patients exhibit on tests of source memory or memory for temporal order can also be seen as related to a specific deficit in episodic memory. Despite these deficits, prefrontal patients perform well on tests of recognition, especially forced-choice, and thus differ from patients with medial temporal lobe damage who exhibit deficits in both recall and recognition. The fact that prefrontal damage leads to specific recall deficits is a concern for studies comparing recall and recognition, because many common etiologies of amnesia, such as Korsakoff's disease, head trauma, and rupture of the anterior communication artery, lead to prefrontal damage. I agree with $\mathrm{A} \& \mathrm{~B}$ that the hippocampus is critical for episodic recall, and I would add that prefrontal cortex is also a crucial part of this system.

To support the idea that recognition deficits can occur despite intact recognition, $\mathrm{A} \& \mathrm{~B}$ present data from nonhuman primate studies demonstrating double dissociations between object recognition and spatial memory (sect. 4.3). However, not only do these tasks differ in terms of recognition versus recall, they also differ in terms of visual object versus spatial memory. Perhaps this dissociation could be viewed in terms of the type of information learned, and not the mnemonic process involved.

The data from neuropsychological patients also fails to make the case that recognition deficits can exist alongside intact recall. $\mathrm{A} \& \mathrm{~B}$ describe patients with focal temporal cortical atrophy that display preserved episodic memory but have deficits in semantic memory. However, the dissociation in these patients is between remote memories (both episodic and semantic) and new learning (Graham \& Hodges 1997). In addition to intact episodic learning, these patients exhibit intact recognition, consistent with the idea that they are losing stored memories yet are able to form new memories. In other cases, because of its role in ventral visual stream processing, temporal lobe damage may result in visual agnosia that could impair visual recognition. However, it has not been clearly demonstrated that these patients would be able to recall newly acquired memories of the same type of information used in recognition tests. The deficit is not one of recognition per se, but a more basic deficit in visual processing. For example, a prosopagnosic patient would have difficulty recognizing faces, but no problem with the recall or recognition of words.

$\mathrm{A} \& \mathrm{~B}$ make the point that recognition itself is composed of multiple processes (sect. 6). Individuals may recognize items because they are consciously recollected (Remembered), a process that shares many properties of episodic recall. Conversely, subjects may recognize items based on familiarity, in the absence of specific recollections (Knowing). A\&B predict that these two types of recognition could be doubly dissociated because they depend on independent brain systems. However, the evidence to date on the remember/know $(\mathrm{R} / \mathrm{K})$ distinction suggests that the relationship between the two is more likely to be redundancy than independence. First, although $\mathrm{R}$ response accuracy can be manipulated without affecting $\mathrm{K}$ response accuracy, changes in $\mathrm{K}$ response accuracy are always accompanied by changes in $\mathrm{R}$ response accuracy. When a manipulation does affect $\mathrm{K}$ responses, it tends to increase both hits and false alarms, suggesting that it affects only the response threshold, not memory (Rajaram 1993). Knowing has only been manipulated independently of remembering under very shallow study conditions when subjects must make rapid judgments and memory is weak (Gregg \& Gardiner 1994). Under these circumstances, knowing may reflect perceptual fluency and, like priming, may not depend on the medial temporal lobe at all.

Remembering and knowing were first conceptualized as indices of episodic and semantic memory, and thus knowing would be redundant with remembering (Tulving 1985a). One could not remember an episode without also knowing the component facts that make up that episode. The fate of items receiving $\mathrm{R}$ and $\mathrm{K}$ responses over time argues for redundancy as well. In this study, subjects were tested after a 10-minute delay, and again after one week (Knowlton \& Squire 1995). For each subject, we found that a large proportion of items receiving $\mathrm{R}$ responses at 10 minutes went on to receive $\mathrm{K}$ responses after 1 week. There was virtually no conversion of $\mathrm{K}$ items to $\mathrm{R}$ items over the week. Also, the $\mathrm{R}$ to-K conversion rate was so great that it could only be accounted for if all items that were given an $\mathrm{R}$ response also had the capacity to elicit a K response (Knowlton 1998).

If recall or recollection cannot occur independently of processes underlying familiarity, it suggests that the brain structures that subserve recognition are also critical for encoding for subsequent recall. However, it is likely that different subsystems of the medial temporal lobe subserve memory for different modalities (visual/object vs. spatial/contextual). Recall, especially episodic recall, depends additionally on other brain systems, including prefrontal cortex, that are important for the reconstruction of memories from one's personal past.

\section{Consideration of the drive properties of the mammillary bodies solves the "fornix problem"}

\section{E. E. Krieckhaus \\ Department of Psychology, New York University, New York, NY 10003 krieck@worldnet.att.net}

Abstract: Fornix problem: Why do lesions of the fornix, which connects the hippocampus (HF) to the medial mammillary nucleus (MMN), often cause no deficits in tasks severely affected by lesions of HF or MMN? Solution: The direct HF feedback to antero ventral (AV) thalamus (MMN $\Rightarrow \mathrm{AV} \mathrm{HF}$ ), which is blocked by MMN lesions but not fornix lesions, is sufficient for nonscene-relevant consolidation.

As a long-time proponent of the importance of the medial mammillary nucleus $(\mathrm{MMN})$ in higher cognitive processes (Krieckhaus 1964), I was pleased to see the conclusion of Aggleton and Brown (A\&B) that MMN is necessary for explicit consolidation of memory. As they acknowledge, if one argues that MMN is implicated in consolidation, either because MMN receives its only cognitive input from hippocampus (HF) or because of MMN pathology in Korsakoff psychosis, the crucial fornix problem emerges: Why are there often no consolidation deficits with lesions of fornix that connects $\mathrm{HF}$ with MMN?

Because of fractionation of disciplines, the intrinsic importance of $\mathrm{MMN}$ in terms of physiological psychology and drive (D) has been overlooked. More generally, as A\&B recognize, it is currently difficult to conceptualize how MMN plays any definitive role in cognition. I hope to show that only by the resurrection of the landmark work of the great American psychologist Clark Hull (1943) can the role of MMN in cognition in general, and consolidation of memory in particular, be understood. Specifically, to incorporate drive (D) into physiological psychology we must use Hull's equation $(\mathrm{E}=\mathrm{H} \times \mathrm{D})$, which synthesizes cognitive habit strength $(\mathrm{H})$ and motivational D, to compute the strength of an action/reaction potential (E). By considering the hypothalamic nature of $\mathrm{MMN}$ as mediating Hull's D, the difficulty in understanding the function of $\mathrm{MMN}$ in consolidation of explicit memory is clarified in a way that may solve the fornix problem.

1. Neural instantiation of Hull's learning equation. In Figure $1, \mathrm{H}$, as long-term memory, is instantiated as neural connections in parietal temporal occipital cortex (PTO). Furthermore, $\mathrm{H}$ is largely focused into MMN, the hypothalamic node of the Papez circuit (Pap) loop: subiculum (SUB) of $\mathrm{HF} \Rightarrow \mathrm{MMN} \Rightarrow \mathrm{AV} \rightleftharpoons$ ingulate $\Rightarrow$ UB (Krieckhaus et al. 1992; Ungerleider \& Mishkin 1982). $\mathrm{E}$ is instantiated in cingulate cortex, which directs action at all levels of the motor system (Dum \& Strick 1993), and is broadcast out of Pap. Now that we see that Pap lies between H (PTO) and E (cingulate), and that D has not yet been introduced, we conclude that Pap should mediate D. 


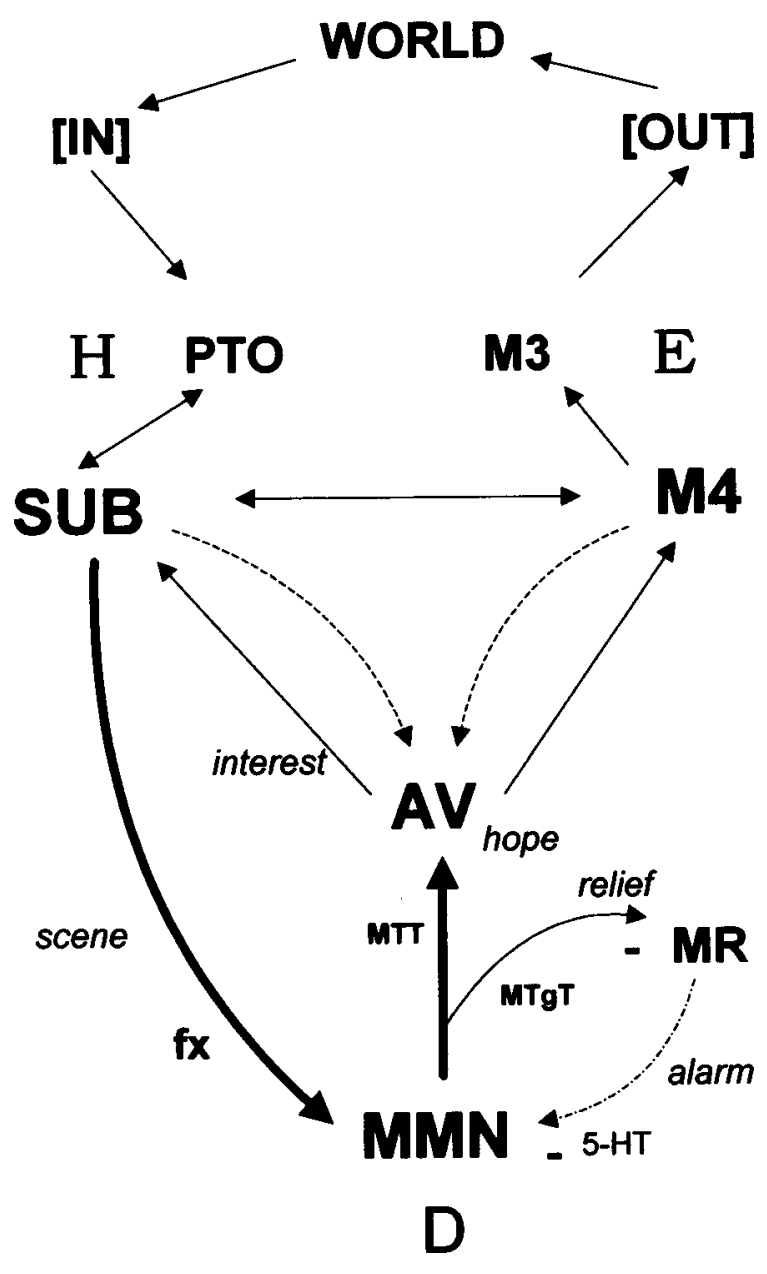

Moreover, because D is naturally associated with hypothalamic functions, and MMN is clearly hypothalamic, then MMN mediates D.

2. Four features of thalamic anatomy necessary for solution of the fornix problem. (1) The architecture of the mammalian thalamus is well known: Feedback from cortex is largely on the distal portion of the dendrites of the principle neurons of thalamus. In most cases, principal neurons project back to the same discrete portion of cortex that supplies their cortical input. The external input (e.g., retina) is largely excitatory on the proximal dendrites of principal neurons within glomeruli. These proximal glomeruli also enclose dendrites of GABAergic inhibitory interneurons.

(2) Functionally, the morphology of thalamic neurons allows distal dendritic input to gain access to the soma easily (Bloomfield \& Sherman 1989; Cai \& Lo 1996).

Assumption: Proximal gates distal. Unlike the statements above, which are descriptions of well-documented neural connections or functions, the following gating assumption - though quite plausible from (2) - has not been proposed, as far as I know. Given the ease with which distal potentials can reach the soma, I assume that often, the function of proximal input is not to transmit its input pattern to drive thalamus and thus cortex, but rather only to selectively regulate, modulate, or gate the potential of the distal cortical feedback to invade the soma of principal neurons, and thus be sent back to cortex.

(3) To understand the unique properties of Pap's AV, note that the thalamus may be simply dichotomized: Posterior nuclei receive direct sensory input (e.g., retina) and project to posterior cortex (PTO), whereas anterior nuclei receive indirect feedback from the
Figure 1 (Krieckhaus). Structure and function of Papez circuit and its computation of $\mathrm{E}$ from $\mathrm{H}$ and $\mathrm{D}$.

The neural solution of the Hull equation, $\mathrm{E}=\mathrm{H} \times \mathrm{D}$, is in the flow of information through the mammalian forebrain, in particular through Pap, which is depicted - synapse by synapse - in the lower portion of the Figure. Pap consists of: $[\mathrm{SUB} \Rightarrow \mathrm{MMN} \Rightarrow \mathrm{AV} \Rightarrow$ $\mathrm{M} 4 \Rightarrow \mathrm{UB} . .$.

At the top of the picture is the external WORLD as it affects the primary sensory input systems, [IN], to the left. [IN] projects into the PTO posterior association cortex, which embodies H. At the upper right is output from the forebrain. M4 of cingulate cortex projects to M3 of cingulate. In turn, M3, as E, directs action at all levels of the motor system, [OUT], shown at the upper right. To close the classical connections of Pap as a circuit, SUB and M4 (as all limbic cortices) are significantly interconnected, as indicated with the double-headed arrow between them. The hypothalamic function of MMN as mediating D is depicted at the bottom. Opiate neurons comprising MMN change their firing pattern as a function of concentrations of various humors, particularly 5-HT, the alarm humor from MR, which is inhibitory, as shown at the lower right.

Pap's thalamus, AV, in the center, receives its subcortical cognitive signal exclusively from MMN, via MTT, and provides "interest" to SUB to consolidate memory as $\mathrm{H}$ in PTO. MMN, via MTT, provides "hope" for voluntary action through AV and M4. The other MMN function is to provide relief from alarm, via MTgT, by inhibiting MR from releasing 5-HT. With the depiction of scene arriving at $\mathrm{MMN}$ from $\mathrm{fx}_{\mathrm{x}}$, the function of each connection of Pap is specified, as shown. Note in Figure 2 that, quite unlike any other thalamic nucleus, AV receives its inputs (on both proximal and distal dendrites) from the same cortex - SUB.

Abbreviations: $\mathrm{E}=$ reaction potential; $\mathrm{H}=$ habit strength; $\mathrm{D}=$ drive; Pap $=$ Papez circuit; $\mathrm{SUB}=$ subiculum; $\mathrm{MMN}=$ medial mammillary nucleus; $\mathrm{AV}=$ antero ventral thalamic nucleus; PTO $=$ parietal temporal occipital cortex; 5 -HT $=$ serotonin; $\mathrm{MR}=$ medial raphe; $\mathrm{MTT}=$ mammillo thalamic tract; $\mathrm{MTgT}=$ mammillo tegmental tract; $\mathrm{fx}=$ fornix. forebrain via four loops, and project to anterior cortex (frontal and cingulate).

(4) Of these four indirect forebrain feedback loops informing anterior cortex, only one, Pap, reveals the following highly eccentric thalamic architecture: Via fornix and MMN, AV receives its "external" input from just that same bit of cortex to which AV projects, namely SUB of HF. In contrast, the other three loops receive their feedback indirectly from large portions of cortex. Thus AV receives the same input on both its distal (directly from SUB) and proximal (indirectly from $\mathrm{SUB}$, via fornix and $\mathrm{MMN}$ ) dendrites.

The fornix problem is now resolved by the fortuitous simplicity of the inputs into AV - just two, SUB feedback distally and indirect SUB input via fornix and MMN proximally (see Fig. 2 ). From A\&B we know that lesions of MMN or MTT cause severe deficits in consolidation, and from AV architecture, we know that the lesions eliminate most of the excitation on AV's proximal dendrites. This allows the now dominant inhibition from the interneurons of AV to choke off the potential of distal SUB feedback to drive AV. Therefore, we have the critical question: Is the consolidation deficit attributable to loss of SUB feedback or to loss of input from SUB via fornix and MMN with a combination of scene and D? Because fornix lesions cause no deficits in consolidation in general, it is apparently SUB feedback that is critical, which explains why fornix lesions do not generally cause deficits in consolidation. However, as stressed by A\&B, fornix lesions do cause severe deficits in scenerelevant consolidation. Thus, for such consolidation, SUB requires that its own output (indirectly via fornix and $\mathrm{MMN}$ ) modulate or gate the transmission of its distal feedback into AV.

In line with other hypothalamic outputs of MMN, the term "in- 
Commentary/Aggleton \& Brown: Episodic memory, amnesia,

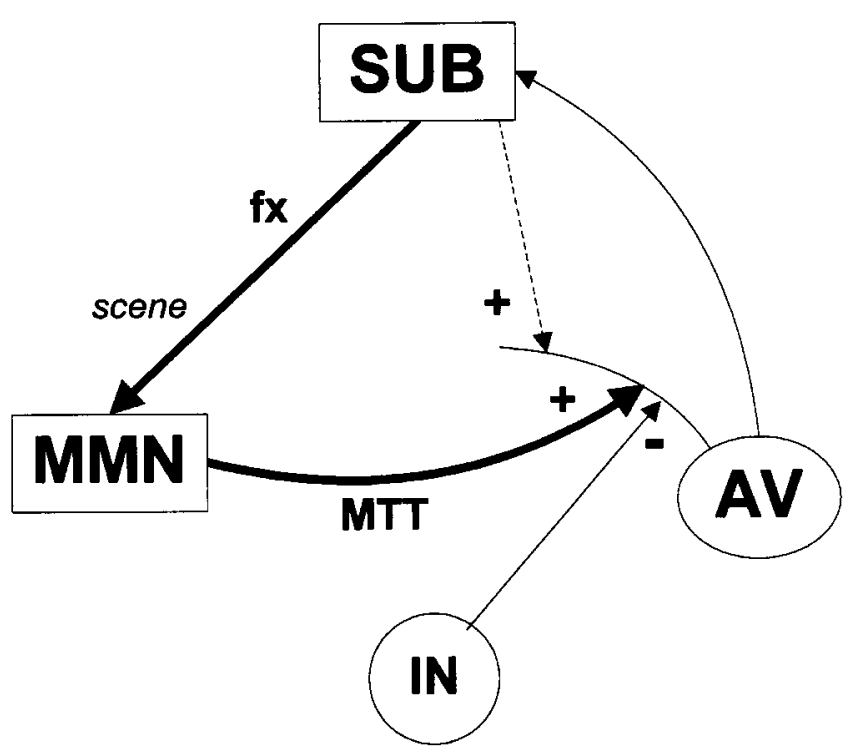

Figure 2 (Krieckhaus). Effect of lesions of FX or MTT.

Figure 2 is a simplification of Pap's connections presented in Figure 1. For abbreviations see caption of Figure 1. Added here is the detailed anatomical relationship between direct cortical feedback from SUB on distal dendrites of $\mathrm{AV}$ and indirect SUB input, via fornix and $\mathrm{MMN}$, on proximal dendrites of AV. The GABAergic, inhibitory interneurons (IN) are shown antagonizing the excitatory MMN input proximally on AV. Lesions of MTT, which materially decrease proximal excitation, allow the inhibition from interneurons to choke off the distal inputs from SUB. Because the lesions that block SUB feedback from penetrating the soma of AV produce severe deficits in consolidation, this feedback is apparently necessary to determine the firing pattern of AV. Because $\mathrm{fx}_{\mathrm{x}}$ lesions, which eliminate the only other source of cognitive input to $\mathrm{AV}$ (via MMN), cause no deficits in consolidation (in general), it is clear that SUB feedback, via AV, is also sufficient for consolidation. However, because fornix lesions cause severe deficits in scene-relevant consolidation, such consolidation by SUB apparently requires SUB's distal AV thalamic input to be gated or modulated proximally by SUB's own indirect output via fornix and MMN.

terest" best captures the drive-like functional significance of this hypothalamic MMN input (via AV) onto SUB, which is necessary for scene-sensitive consolidation - to pay attention to what is of interest and let the rest go by.

In conclusion, consideration of the drive properties of hypothalamic MMN in Hull's formulation, taken with the remarkable architecture of $\mathrm{AV}$, solves the fornix problem raised by $\mathrm{A} \& \mathrm{~B}$ and suggests that the gating assumption (proximals gate distals) is true, at least for Pap - and perhaps for primary sensory input as well.

\section{Thalamic amnesia and the hippocampus: Unresolved questions and an alternative candidate}

\author{
Robert G. Mair, Joshua A. Burk, M. Christine Porter, \\ and Jessica E. Ley
}

Department of Psychology, University of New Hampshire, Durham, NH 03824. \{rgm; jaburk; mcporter; jeley\}@christa.unh.edu

\begin{abstract}
Aggleton \& Brown have built a convincing case that hippocampus-related circuits may be involved in thalamic amnesia. It remains to be established, however, that their model represents a distinct neurological system, that the distinction between recall and familiarity captures the roles of these pathways in episodic memory, or that there are no other systems that contribute to the signs of amnesia associated with thalamic dis-
\end{abstract} ease.

Aggleton \& Brown (A\&B) have synthesized an impressive body of evidence to make a case for their theory relating episodic memory to two parallel pathways linking hippocampus and perirhinal cortex to thalamus. They have avoided some of the pitfalls of reification by focusing on the localization of lesions that impair episodic memory rather than episodic memory per se. Nevertheless, their emphasis on a construct like episodic memory and its relationship to an extended neural circuit raises several questions that are not fully addressed by their review.

1. Are the pathways outlined reasonably construed as a discrete neurological system? The hippocampal projections through fornix to thalamus are well established and (as the authors note) represent pathways that once dominated speculation about the neurological basis of diencephalic amnesia. The main contribution of A\&B's model is the emphasis placed on a projection from perirhinal cortex to the mediodorsal nucleus and its supposed involvement in judging the familiarity of sensory stimuli. It is this projection that represents the most questionable part of their model. Although this projection has been described in monkeys, it is not clear how prominent it is compared to other connections of the mediodorsal nucleus or of perirhinal cortex (Amaral 1987; Jones 1985; Suzuki 1996a), and it apparently has not been demonstrated in the rat (a species cited for much of the supporting behavioral evidence). In describing the transport of wheatgerm agglutinin-horseradish peroxidase from the rat mediodorsal nucleus, Groenewegen (1988) described signs of anterograde and retrograde transport in prefrontal cortex and retrograde transport in prepiriform cortex, periamygdaloid cortex, the lateral entorhinal area, the infralimbic area, and subicular cortex, but not in perirhinal cortex. Although A\&B emphasize the parallel and separate nature of the thalamic projections of hippocampus and perirhinal cortex, the hippocampal formation in fact has well-established connections with the mediodorsal nucleus that travel directly from subiculum and indirectly through striato-pallido-thalamic pathways (Groenewegen 1988). We are aware of no evidence for the projection from the mediodorsal nucleus to perirhinal cortex indicated in A\&B's Figure 1.

2. Does the construct of "episodic memory" and the abstract distinction between recall and familiarity capture the essence of what is impaired by lesions affecting the hippocampal- and perirhinal-related pathways? There is, of course, a great deal of controversy and active research interest in this issue. Although there are other examples that seem difficult to reconcile with A\&B's model, we will focus on recent work in our lab. We have found two tasks, DMS trained with retractable levers and olfactory continuous DNMS, that would seem to require episodic memory but were unaffected by lesions destroying hippocampus or the mediodorsal nucleus (Burk \& Mair 1998a; Mair et al. 1998; Zhang et al. 1998). It would seem to follow that either these tasks are mediated equally well by both the hippocampal- and perirhinal-systems (and are thus little affected by lesions of only one) or they are mediated by other pathways in the brain. If the first pos- 
sibility is true, then one must wonder why DNMS trained in the radial maze is impaired by comparable hippocampal lesions (Mair et al. 1998). Why would perirhinal-related pathways be sufficient to mediate DMS trained with retractable levers but not DNMS trained in an automated radial arm maze?

3. Are there other candidate systems that might also contribute to the signs of anterograde amnesia that have been associated with diseases affecting the diencephalon? There are at least two other systems in thalamus that have been linked to amnesia: the mediodorsal nucleus and its projections to prefrontal cortex (Markowitsch 1982; Victor et al. 1989) and the intralaminar nuclei (ILn) and related nonspecific nuclei within the internal medullary lamina and midline areas of thalamus (Mair 1994; Mair et al. 1979; Malamud \& Skillicorn 1956; Mennemeier et al. 1992; von Cramon et al. 1985).

Studies of animal models of amnesia have provided several lines of evidence implicating the ILn as an important site of pathology in the Wernicke-Korsakoff Syndrome, a common cause of diencephalic amnesia thought to be caused by thiamin deficiency. Earlier studies showed that the ILn are vulnerable to the effects of pyrithiamine-induced thiamin deficiency and that the extent of ILn damage correlates with the extent of behavioral impairment in rats recovered from this treatment (Langlais et al. 1996; Mair et al. 1988). Thus rats that recover from the acute effects of pyrithiamine-induced thiamin deficiency with lesions involving extensive areas of the ILn tend to perform poorly on DMS and DNMS tasks used to measure memory function (Knoth \& Mair 1991; Langlais \& Savage 1995; Robinson \& Mair 1992). The role of the ILn in these impairments has been verified by studies showing comparable behavioral deficits in rats with electrolytic or excitotoxic lesions affecting these nuclei (Burk \& Mair 1998a; Mair 1994; Mair et al. 1998; Zhang et al. 1998). The involvement of hippocampal-related pathways in these deficits seems inconsistent with evidence that similar impairments are not observed following lesions of the mediodorsal nuclei, fornix, mammillary bodies, hippocampus, or the laterodorsal nuclei (Burk \& Mair 1998a; 1998b; Mair 1994; Zhang et al. 1998). We recently completed a series of studies that demonstrated distinctive patterns of impairment in rats with lesions of the ILn, the hippocampus, or the prefrontal cortex on different measures of remembering: DMS trained with retractable levers, several versions of DNMS trained in automated radial arm mazes, and go/no go olfactory continuous DNMS (Burk \& Mair 1998a; Koger \& Mair 1994; Mair et al. 1998; Porter \& Mair 1997; Zhang et al. 1998). We interpret these results as evidence of functionally distinct impairments of memory associated with thalamic, hippocampal, and prefrontal pathology.

The questions raised in this commentary should only reinforce the importance of understanding the effects of lesioning hippocampal-related pathways in thalamus and their possible contributions to diencephalic amnesia. A\&B are well justified in pointing out the potential significance of these pathways for memory and they have presented a model that should help focus thinking about this issue. In our view, the available evidence is insufficient to support the claims of the model or to rule out the possible involvement of other systems in diencephalic amnesia.

\section{Gestalt view of the limbic system and the Papez circuit - another approach to unity and diversity of brain structures and functions}

\author{
Hans J. Markowitsch \\ Department of Physiological Psychology, University of Bielefeld, D-33501 \\ Bielefeld, Germany. hjmarkowitsch@post.uni-bielefeld.de \\ www.psychologie.uni-bielefeld.de/ae/ae14/index.htm
} Abstract: The idea of distinct brain systems for the processing of episodic
and other forms of memory is welcome. The two brain systems actually
proposed however, appear to be stripped of further existing connections
and could be integrated with one another. If integrating them, it seems
more logical to propose one enlarged system of limbic structures whose
individual components make partly different contributions to the forms of
memory under discussion.

Aggleton \& Brown's (A\&B’s) proposal is welcome, as it emphasizes that the discussion of anterograde amnesia should not focus only on the hippocampal region and that there may be important differences on the anatomico-behavioral levels between the episodic and other memory systems (semantic, declarative memory) (Tulving \& Markowitsch 1998). Nevertheless, there seems to be something artificial about A\&B's major distinction between an "extended hippocampal system" (which is in fact nothing other than the old Papez [1937] circuit) for encoding of episodic information and a "perirhinal-mediodorsal thalamic system" for familiarity judgments. The division into these two systems (exemplified by A\&B's Figs. 1 and 2) ignores the existence of a multitude of additional connections with these structures and consequently, their integrating role in memory processing (e.g., basal forebrain structures, amygdala). A\&B make their proposed division immune to attack by acknowledging that in the large majority of cases of amnesia, both systems are affected, and by limiting their second system to the thalamic mediodorsal nucleus (proper) and the perirhinal cortex (proper).

From the clinical literature it is hardly possible to exclude perirhinal damage in cases with medial temporal lobe pathology or midline nucleus or mamillothalamic tract damage in cases with medial thalamic pathology. On the other hand, it is also nearly impossible to find patients with selective bilateral mediodorsal thalamic damage (Markowitsch 1982; 1988; 1992) or selective bilateral perirhinal damage.

Moreover, the circuitry in Figures 1 and 2 either ignores the existence of further prominent connections (e.g., between the amygdala and the mediodorsal thalamus) or simplifies and hides them by mentioning (for example) a fornix pathway from the hippocampus to the prefrontal cortex. If so, this pathway would be bidirectional and would not involve major portions of the prefrontal cortex but basal forebrain regions such as the medial septum and the hippocampal formation (subicular cortex). Though the comparability (in degree and severity) of the memory deficits after bilateral basal forebrain system damage and bilateral damage of the two other conventionally defined memory systems (medial diencephalic and medial temporal lobe system) is disputed (DeLuca \& Diamond 1995; Diamond et al. 1997), there are at least subgroups of basal forebrain lesioned patients with amnesias of a severity comparable to that seen after major damage to one or the other (Böttger et al. 1998; Diamond et al. 1997; Von Cramon et al. 1993).

Consequently, the issue becomes less about the differences between the two somewhat artificially divided systems (cf. Fig. 1), and more about the degree and diversity of involvement of limbic system structures as such, and the consequences of disconnection that have been repeatedly emphasized in so-called global amnesia. First, we know that a brain region never acts on its own, but depends on input and output from other regions. This statement is trivial and is often taken as a persiflage by arguing that no brain 
region is more than six synapses away from another one. Such a view throws the baby out with the bath water. Functional imaging has revealed that the pathology after a local injury, infarct, or degeneration as demonstrable by static imaging may extend to remote, diverse, and dispersed additional loci (Markowitsch et al. 1997). Nevertheless, these further affected regions usually have intimate connections to the local focus.

Second, amnesia for episodic information is to a great extent amnesia for emotionally laden information (Cahill et al. 1995; Markowitsch 1994; Markowitsch et al. 1993). That is, structures such as the amygdala (which projects to the mediodorsal nucleus) and the septal nuclei (Von Cramon et al. 1993) contribute substantially to a successful encoding of episodic information. Given this interconnectivity, it is quite likely that the perirhinalmediodorsal memory system is centrally engaged in episodic memory encoding. In fact, seen from another level, Tulving and Markowitsch's (1998) model would likewise predict that the perirhinal-mediodorsal system must be engaged before the extended hippocampal system, as the model assumes (see their Fig. 1) that episodic information must pass through the semantic memory system before becoming successfully encoded. Hence the division into the memory systems proposed by $\mathrm{A} \& \mathrm{~B}$ does have some face validity, especially based on findings obtained from animal research; but it is certainly inadequate in light of the intimate interdependencies of the many structures subsumed under the heading "limbic system" (Markowitsch 1999) and engaged in episodic memory functions.

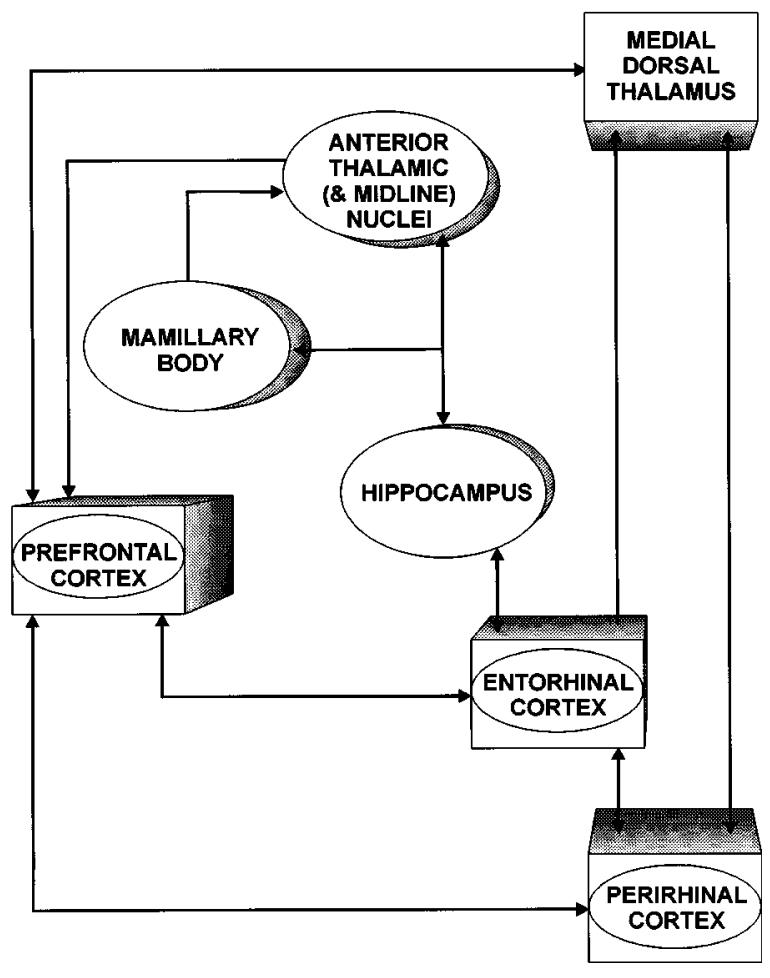

Figure 1 (Markowitsch). Realignment of the structures and connections of the two memory systems proposed by A\&B. The figure contains the "perirhinal-mediodorsal system" together with the "extended hippocampal system," which can be seen as embedded in the circuitry of the perirhinal-mediodorsal system. The connectivity follows exactly that of A \& B's Figures 1 and 2 but it omits a few structures and connections (along with the occasional emphasis on the relative strength of a given pathway). Regions that belong to the "perirhinal-mediodorsal system" are rectangular; regions belonging to the "extended hippocampal system" are oval; regions belonging to both circuits contain both shapes.
ACKNOWLEDGMENTS

I thank Eva Böcker for help with the drawing of Figure 1. My work was supported by the DFG through grants Ma 795/24 and Ma 795/25.

\section{What are the functional deficits produced by hippocampal and perirhinal cortex lesions?}

\author{
A. R. Mayes ${ }^{a}$, R. van Eijk ${ }^{b}$, P. A. Gooding ${ }^{b}$, C. L. Isaac ${ }^{a}$, \\ and J. S. Holdstock ${ }^{a}$ \\ ${ }^{a}$ Department of Clinical Neurology, Royal Hallamshire Hospital, University \\ of Sheffield, Sheffield S10 2JF, England; b Department of Psychology, \\ University of Strathclyde, Glasgow G1 1QE, Scotland. \\ \{a.mayes; c.isaac; j.holdstock\}@sheffield.ac.uk \\ patricia.gooding@strath.ac.uk
}

Abstract: A hippocampal patient is described who shows preserved item recognition and simple recognition-based recollection but impaired recall and associative recognition. These data and other evidence suggest that contrary to Aggleton \& Brown's target article, Papez circuit damage impairs only complex item-item-context recollection. A patient with perirhinal cortex damage and a delayed global memory deficit, apparently inconsistent with A\&B's framework, is also described.

This comment focuses on Aggleton \& Brown's (A\&B’s) proposal that selective Papez circuit lesions in humans disrupt free recall and leave item recognition intact and A\&B's interpretation of this effect. We also comment briefly on evidence that selective perirhinal cortex system lesions have different effects on human memory.

Selective Papez circuit lesions are extremely rare in humans and normal or relatively preserved item recognition is not always seen following apparently selective damage. It remains to be shown whether such patients have additional cryptic damage outside the Papez circuit. We have examined extensively one patient with relatively selective bilateral hippocampal damage who often shows completely normal recognition in the face of impaired recall. On many item recognition tests, she scores as well as or better than matched control subjects, although she has occasionally been impaired. Her performance has been within the normal range on Yes/No and Forced Choice item recognition tests at both short and long delays of one day or more. Relative preservation is not a simple function of difficulty because she has performed above the control mean on a hard object recognition test matched for difficulty with an object free recall test at which she was very impaired.

Several features of this patient's performance warrant comment. First, she is impaired at associative, but not item, recognition. Thus, she was greatly impaired at an object-location recognition test that normal subjects found easier than the object recognition test at which she was unimpaired. She was also impaired at temporal order, face-voice, word-meaning, and animal picture-occupation name associative recognition. Second, her story recall, but not her story recognition ability, declined abnormally fast between delays of 15 seconds and 10 minutes. Third, using a modified process dissociation procedure (PDP) with both famous faces and names tasks, we found not only normal item recognition, but normal recollection.

$A \& B$ argue that patients like ours have selective item-context memory (recollection) deficits. This argument assumes that normal performance on some recognition tasks depends on familiarity alone, which is contrary to the widely held view that item recognition depends on both recollection and familiarity. The target article suggests that Papez circuit damage disrupts the contextitem retrieval underlying free recall. If this is identical to the itemcontext retrieval manifested in recognition, recollection cannot contribute to item recognition tasks at which our patient is normal. However, Hasselmo and Wyble's (1997) recent model of hippocampal function shows that this identity may not hold. They modelled recall as the retrieval of items from context cues and recognition as the retrieval of context from item cues (familiarity 
played no role). Only aspects of the study situation appearing repeatedly were counted as context, so features appearing uniquely with a studied item were excluded. Giving the model a "biochemical lesion" disrupted recall, but not recognition, because such repeated context was well learned. The model shows that recollection involving repeated context features can break down for recall, when it does not for item recognition.

$\mathrm{A} \& \mathrm{~B}$ suggest that recollection does not contribute to recognition because this is not usually affected by study-test context shifts, whereas recall is. Although this was not modelled by Hasselmo and Wyble, it is plausible that such modelling would show minimal effects of context shifts on item recognition as these resemble lesions of the model. Hence, stronger evidence for the assumption that recollection does not contribute to recognition needs to be provided.

We are also unconvinced that the familiarity, preserved after hippocampal lesions, cannot be mediated by enhanced item fluency (priming) being automatically used to attribute familiarity to previously encountered items. This is consistent with some amnesics performing at chance on recognition tests because attributing familiarity may depend not only on enhanced fluency of item processing, but also on being able to recollect sufficiently about the study context when they are unable to do this. Forced choice tasks are no more likely than Yes/No tasks to force subjects to use fluency to make oldness attributions when memory for the study context is largely absent.

In contrast to global amnesics, our hippocampal patient makes oldness attributions normally (familiarity) because she can also recognize associations between items and repeated contextual features normally as shown by our PDP results. However, she does not recognize more complex associations between two or more items and these repeated contextual features as is suggested by her impaired performance on association recognition tasks. Detailed analysis of what she recollects when she recognizes items suggests that she rarely recalls specific associations between a target item and one or more other items (or thoughts) together with the repeated context features.

We think that our patient and similar ones show preserved item priming and item-repeated context feature memory, but do not consolidate complex item-item-repeated context feature associations into long-term memory normally after single presentations. Support for this view is the accelerated loss of story free recall shown by our patient. Unlike story recognition, which is mediated by simple item-repeated contextual feature association retrieval and familiarity, recall requires retrieval of these more complex kinds of association. The deficit may be confined to the consolidation of complex associations where the components are represented in distinct neocortical regions.

Selective perirhinal cortex lesions have never been reported in humans. We have, however, studied a patient with extensive bilateral damage to what should be this region in humans. This patient has an intact hippocampus although damage extends into the temporal association cortex at the temporal pole. She shows preserved recognition and recall except for faces at delays of $30 \mathrm{~min}-$ utes, but forgets rapidly over delays of a few weeks, at which time recall and recognition deficits appear. Such cases suggest that this brain region may not have the functions one might expect from the animal studies referred to in the target article. Assuming that face recall, like face recognition, is impaired early, our patient shows no recall/recognition dissociation, but a delayed global memory deficit. Future work must identify the conditions that trigger this syndrome and its functional basis.

\section{How do animals solve object-recognition tasks?}

\author{
Dave G. Mumby \\ Department of Psychology, Concordia University, Montreal, Quebec, Canada \\ H4B 1R6. mumby@vax2.concordia.ca
}

Abstract: This commentary reviews recent evidence that some hippocampal functions do not depend on perirhinal inputs and discusses how the multiple-process model of recognition may shed interpretive light on previous reports of DNMS reacquisition deficits in pretrained subjects with hippocampal damage. Suggestions are made for determining whether nonhuman subjects solve object-recognition tasks using recollective memory or familiarity judgments.

Aggleton \& Brown $(\mathrm{A} \& \mathrm{~B})$ have abandoned the widely held view that there is a single "temporal-lobe memory system," suggesting instead that the perirhinal cortex and hippocampal formation are functionally independent. Their observation that this may be considered a "surprising" feature of their model gives insight to the kinds of pretheoretical assumptions that have helped to maintain the unitary-system view for so long in the first place for it has not been on the basis of behavioural evidence that the perirhinal cortex and hippocampal formation are functionally interdependent, but rather on anatomical evidence of strong reciprocal connections between these two structures. Perhaps what should surprise us is how much has been previously inferred about the functional relationship the perirhinal cortex shares with the hippocampus from this one feature of its extrinsic circuitry. The existence of the requisite connections does not tell us whether two structures depend on each other for their respective functions. Nor is there any reason to assume that the degree of functional similarity between two structures is proportional to the density of connections they share. The latter assumption seems to be the main reason why many believe information-processing within the hippocampal formation requires inputs from the perirhinal cortex, despite a dearth of behavioural evidence to support this view.

We recently reported a double-dissociation following lesions of the perirhinal cortex and hippocampal formation, consistent with $A \& B$ 's conclusion that these two structures are functionally independent: Rats with perirhinal lesions were impaired on an object-based delayed nonmatching-to-sample (DNMS) task but performed normally on delayed matching-to-place (DMTP) in a water maze, whereas rats with hippocampal lesions were impaired on DMTP, but not DNMS (Glenn \& Mumby 1996). Each rat was tested on both tasks, so the dissociations were apparent within subjects, and therefore, cannot be attributed to differences in lesion extent - a potential caveat whenever ostensible dissociations occur across separate studies, as was the case with our previous demonstrations of this particular double-dissociation (Glenn \& Mumby 1998; Mumby \& Pinel 1994; Mumby et al. 1992). The DNMS and DMTP tasks employed the same retention delays (ranging from 4 to $300 \mathrm{sec}$ ), and (quasi) trialunique stimuli were used in both tasks, similarities that suggest that the dissociations occurred because one task required memory of object identity, whereas the other required memory of a spatial location.

Although our findings are consistent with A\&B's conclusion that spatial-information processing by the hippocampus does not require inputs from perirhinal cortex, there are reports that perirhinal lesions disrupt performance of some allocentric-spatial tasks (Liu \& Bilkey 1998c; 1998b; Wiig \& Bilkey 1994). But demonstrating that either perirhinal or hippocampal lesions can impair performance of the same spatial-memory task does not reveal why the task is sensitive to perirhinal damage. Any task can fail for a variety of reasons. Given the lack of perirhinal lesion effects on allocentric tasks in our experiments and in those of $\mathrm{A} \& \mathrm{~B}$, it is likely that the deficits observed by Bilkey and his colleagues were caused by some other feature of their tasks. Do perirhinal lesions cause 
deficits by depriving the hippocampal formation of critical inputs? This question can potentially be addressed by assessing the effects of crossed-unilateral lesions of these two structures, combined with commissurotomy, on performance of the task that is disrupted by bilateral lesions of either structure (assuming the typical situation where unilateral lesions of either structure spare performance of the task).

The suggestion that subjects may solve the DNMS task by using either recollective memory or a familiarity judgment raises the question of whether members of a particular species are predisposed to use one strategy rather than the other. The answer is important if we are to compare findings across species, as the superficial similarities in the DNMS tasks for humans, monkeys, and rats do not justify the assumption that all three species solve them the same way. If a lesion impairs a subject's capacity for solving the task using the preferred strategy, how might this affect postsurgery performance? Some of our results are consistent with the hypothesis that hippocampal lesions disrupt a preferred strategy for solving the DNMS task, while leaving intact the capacity for an alternative solution. Following hippocampal ablation, rats that had received presurgery training were impaired in reacquisition at the 4 -sec retention delay (Mumby et al. 1992; 1996). We originally interpreted this as reflecting a nonmnemonic procedural impairment because all rats soon overcame the deficits, and thereafter continued to perform as well as controls at much longer delays. But another possibility that stems from the multiple-process model of recognition is that these transient deficits occurred as hippocampal rats discovered their inability to solve the task the same way as before surgery and had to switch to a less-preferred but still effective alternative.

How could one go about determining whether a subject is solving the DNMS task using recollective memory or familiarity judgments? Two points are worth noting here: First, A\&B's distinction between familiarity and priming on the basis of conscious awareness being part of the former but not the latter is of little utility when considering how nonhuman animals solve a task because we can neither confirm nor disconfirm conscious awareness in nonhumans. Second, in the absence of conscious awareness there may be little or nothing to distinguish the processes underlying familiarity judgments from those that occur in instances of repetition priming. A general strategy for asking whether a recognition task for nonhuman animals engages processes that are similar to those of recollective memory is to examine the effects of task manipulations that are known to have different effects on recollective memory and familiarity judgment in humans. For example, distraction during the retention interval disrupts the performance of humans on explicit-memory tests but not implicit-memory tests (Graf \& Schacter 1985). DNMS performance in rats (Mumby, unpublished data) and monkeys (Zola-Morgan \& Squire 1985b) is disrupted when the subjects must perform a distraction task during the retention interval. Does this mean that rats and monkeys use explicit memory to solve the DNMS task? The effect of distraction by itself might not be very convincing, but the evidence could become more compelling if additional manipulations were found to have similar effects on explicit memory in humans and DNMS performance in animals. The same general approach could be used to probe whether two groups of subjects performing at similar levels of accuracy are actually solving the task the same way, or whether brain-damaged subjects that received presurgery training are still solving the task the same way they did before the lesions.

\section{A "presence/absence hypothesis" concerning hippocampal function}

\author{
David J. Murray \\ Department of Psychology, Queen's University, Kingston, Ontario K7M 3L6, \\ Canada.murrayd@psyc.queensu.capavlov.psyc.queensu.ca
}

Abstract: According to a "presence/absence hypothesis," the hippocampus is not necessary for the formation of learned associations between currently present stimuli and responses (as in classical conditioning), but is necessary whenever a stimulus, if it is to activate a particular response, must first activate a memory-representation of something not present in the here-and-now. The distinction between responses made to present stimuli as opposed to (memories of) absent stimuli was first stressed by Romanes (1889), but we find evidence in the target article that supports the relevance of this distinction to our understanding of hippocampal functioning.

In this commentary I shall offer a hypothesis concerning the role the hippocampus plays in memory tasks given to animals or humans. I shall take three findings about the hippocampus, one well known, and two that were new to me until I read Aggleton \& Brown's (A\&B's) target article, and offer these as preliminary evidence for the hypothesis. According to "presence/absence hypothesis," the hippocampus is not necessary for mediating learned responses when the retrieval cue is present in the here-and-now; but if the learned responses require the use of memory representations of absent things or situations, then the hippocampus does become necessary.

(1) The well-known finding is that, in animal subjects, hippocampal lesions do not destroy the ability of the subject to learn classically conditioned responses, disrupt spontaneous recovery, or affect the processes of extinction. Evidence for these statements has been provided in the reviews of hippocampal functioning by O'Keefe and Nadel (1978, pp. 316-24) and, more recently, by Cohen and Eichenbaum (1993). In classical conditioning an animal (or human) learns to make novel responses to what had previously been a stimulus of neutral emotional value; for example, Pavlov taught his dogs to salivate on hearing the clicks of a metronome. Since Pavlov's time, Rescorla (1967) and his colleagues have indicated that the activation of the salivary response appears to depend on the dog's having learned to "expect" food after the metronome has sounded. I find it difficult to see what is wrong with rephrasing this last sentence as follows: "The dog learns to recognize the metronome as a signal that food is about to arrive." All pet owners know the speed with which their cat or dog comes running when it "recognizes" the sound of a can being opened as a signal that food is on the way. What we wish to emphasize here, however, is that the sound of the metronome or of the can being opened is a conditioned stimulus that is "present" and is not imagined by the animal. In contrast with that of animals, much human behaviour is initiated by imagining a stimulus that is not present, but absent. A hungry person can salivate at the thought of a hamburger. Hence, when hippocampal lesions fail to eradicate the formation or elicitation of classically conditioned responses, we must at least consider the possibility that it is because the conditioned stimuli are present in the animal's environment immediately before the conditioned response is activated and that the hippocampus is not necessary for processing information concerning "recognition as" responses to present stimuli, at least in animals.

(2) In section 4.1, third paragraph, A\&B reported that DNMS deficits had followed localized destruction (by the injection of neurotoxins) of hippocampal fields CA1-4, along with the amygdala, in experiments in which monkeys were removed from the apparatus during the retention-interval (Alvarez et al. 1995). On the other hand, Murray and Mishkin (1996) have reported that, if animals with hippocampal lesions similar to those induced by Alvarez et al. were left in the apparatus during the retention interval, they could pick out a new stimulus (from two stimuli, one of which had been seen before) after a delay of as long as 40 minutes. 
A large number of apparatus cues would have remained present for all of the retention interval during Murray and Mishkin's experiment, but would have been absent in the case of the experiment by Alvarez et al. (1995). This finding, which obviously merits further investigation, is consistent with the notion that if the spatial context is always available to the subject during a retention interval, then adequate performance on this task does not require an intact hippocampus to the extent demanded in tasks where the subject is removed, then put back into the apparatus. Agreed, this might be because positional stances may be adopted in the interval, but it may also be because the contrast of a novel stimulus object with the apparatus background is more striking if the background has been available for many minutes beforehand than if it had been absent and suddenly reinstated.

(3) In the first column of Table $1, A \& B$ indicate that hippocampal ablations cause little damage in DMNS tasks but cause significant damage in DMNP tasks. The content of the previous paragraph may indicate that this generalization needs qualifying, but here I wish to focus on the fact that in delayed memory tasks in which the subject must remember objects (DMNS) as opposed to places (DMNP), there is a reinstatement of a spatial background at the time of delayed nonmatching to sample in which two objects (one old, one new) are both present. One of the objects has to be responded to as "new." But in the cases of DNMP tasks, or of spatial alternation in T-mazes, the animal at the choice point has to retrieve from memory information concerning the direction in which to turn. In the DMNS task the animal has to recognize a present stimulus object as new, whereas in the DMNP task it does not have to recognize a present sensory stimulus (a choice point) as new, but only as a starting point, for the self-initiation of a new set of movements (see also Poucet 1993).

The idea that the hippocampus may have evolved for the purpose of facilitating the representation, to the subject, of things, places, and emotions that are not actually "present" in the hereand-now is consistent with the general notion that human intelligence differs from animal intelligence chiefly insofar as humans can imagine absent objects far more easily than animals can, particularly when humans have language to help them do so. The clearest and most detailed statement of this idea has been provided, in my opinion, by Romanes (1889).

A secondary account of Romanes's theory has been given by Murray (in press), but an important aspect of it consists in the distinction made by Romanes between what he called "receptual" and "conceptual" memory. Receptual memory is possessed by many animals, and is used whenever a present stimulus is recognized as having been linked in the past with pleasure or displeasure. Conceptual memory is a more advanced stage in the evolution of memory in which absent things can be represented by sensory imagery (including kinesthetic imagery) or by language.

I propose, therefore, that what really matters in determining the role played by the hippocampus in any task is whether the task involves a present stimulus (to which a "receptual" response from memory can be given) or is one in which the subject must provide, from memory, some intermediate (“conceptual”) response to a present stimulus. The hippocampus, it is proposed, plays a more important role in conceptual memory than in receptual memory.

\section{Episodic memory: It's about time (and space)}

\author{
Lynn Nadel, Lee Ryan, Katrina Keil, and Karen Putnam \\ Department of Psychology, University of Arizona, Tucson, AZ 85721. \\ \{nadel; ryant; keil; kputnam\}@u.arizona.edu
}

Abstract: Aggleton \& Brown rightly point out the shortcomings of the medial temporal lobe hypothesis as an approach to anterograde amnesia. Their broader perspective is a necessary corrective, and one hopes it will be taken very seriously. Although they correctly note the dangers of conflating recognition and recall, they themselves make a similar mistake in discussing familiarity; we suggest an alternative approach. We also discuss implications of their view for an analysis of retrograde amnesia. The notion that there are two routes by which the hippocampus can reactivate neuronal ensembles in the neocortex could help us understand some currently puzzling facts about the dynamics of memory consolidation.

Aggleton \& Brown (A\&B) have done the field a considerable service in pulling together many strands of research and clinical experience to refocus our attention on the broader picture in the study of amnesia. This is not to say that their model is completely correct, but we believe their argument is a much needed correction to the overly narrow emphasis on the "medial temporal lobe system" that has dominated the field in the past $10-15$ years. It is interesting to recall that only 20 years ago the connections between hippocampus and its temporal lobe neighbors such as the entorhinal cortex were largely ignored, and all attention was placed on outputs exiting via the fornix. $A \& B$ do a good job of helping us understand just why the focus switched to the caudal projections, and more importantly, how this change in focus has created more confusion than clarity in explanations of memory and memory disorders. Indeed, the past 10 years can be characterized as a series of debates and controversies that have erupted whenever the "medial temporal lobe system" has met with apparent empirical refutation. In replaying these debates, A\&B clarify the flimsiness of the evidence in favor of the MTL hypothesis and the desperate need for a framework that can encompass the complexity of memory and amnesia. A\&B point the way to what might be such a new approach.

Much of the force behind the MTL hypothesis derives from work with primate models, and what the proponents of this view heralded as the definitive development of an animal model of amnesia. Indeed, some went so far as to characterize this "accomplishment" as one of the major triumphs of recent cognitive neuroscience. One result of A\&B's argument is the demolition of this piece of puffery. It is clear from their review of the relevant work that the animal model, focused as it is on recognition memory (and tasks such as delayed nommatch to sample) falls far short of a realistic model of the complex syndrome of human amnesia. By forcing tasks as disparate as free recall, recognition, and semantic recollection into the same "declarative" framework, the MTL theory has impeded our understanding of the tasks themselves and their neural bases. A similar point can be made regarding the proposed distinction between "explicit" and "implicit" forms of memory: Recognition and perceptual identification, as examples of these two, are distinct in some ways but also share common processes (Graf \& Ryan 1990). Taxonomies of tasks based on a single attribute such as "conscious recollection" necessarily result in oversimplifications.

There are, however, some weaknesses in the A\&B approach. We would like to discuss a few of them briefly: the current lack of clarity concerning the impact of damage restricted to the hippocampal formation; how best to think about notions such as novelty and familiarity; and the retrograde portion of the amnesic syndrome.

There remains a lack of clarity concerning the impact of damage restricted to the hippocampal formation. Although there are compelling reasons to be concerned about hidden pathology in clinical cases, one must take care not to turn this idea into a garbage can for findings inconsistent with one's views. The development of techniques for measuring in vivo brain activity provides 
a new source of data that might help clarify this issue. For example, although recent neuroimaging studies that focus on the encoding of novel stimuli point to potentially important functional differentiations among medial temporal lobe structures, and suggest that hippocampus and parahippocampal regions may play a role in encoding relational or configural aspects of complex scenes (Stern et al. 1996; Tulving et al. 1994), the entorhinal cortex may detect and encode singular novel objects. These studies are consistent with A\&B's review of both the c-fos activation studies in animals and recent rat hippocampal lesion studies (Honey et al. 1998). Given this convergence of results, a finding of impaired object novelty detection in a patient with brain damage apparently limited to the hippocampus itself would cause one to be concerned about hidden pathology. In the absence of evidence confirming more extensive damage, however, one is hard-pressed to disregard these inconsistent cases, unless one chooses to disregard neuropsychological evidence all together.

Although A\&B do an excellent job of pointing out the inaccuracy of lumping recognition and recall together, they fall into a similar trap when they attempt to tackle the notion of familiarity. Their definition of it is too narrow, limited as it is to tasks involving an explicit judgment of "knowing." In fact, the term "familiarity" has been used in many contexts, including the accumulation of multiple similar experiences (as in the case of high-frequency words; Glanzer \& Bowles 1976), single-trial learning (as in the case of recognition; Mandler 1980), and even semantic learning (as in the case of semantic priming; Jacoby 1983). Instead of this limited view, we would suggest that the feeling of knowing (FOK) involves at least two important, and separable, components. First, there must be a representation of the thing to be known, and second, there must be a process of monitoring and/or accessing this representation. Under this conception there could be multiple neural systems involved in representation, each subserving different kinds of knowledge, and each contributing to performance on various tasks, including tasks such as item priming. The explicit feeling of knowing, however, would require monitoring and comparing the attributes of these representations, and might depend critically on involvement of the hippocampal formation.

Finally, there is the matter of retrograde amnesia, about which $A \& B$ say very little. This is a pity in that their reformulated view of anterograde amnesia suggests a very intriguing way to think about retrograde amnesia. There is widespread consensus that the hippocampal formation and neocortex interact over time after memories are initially stored. One popular view (e.g., McClelland et al. 1995) is that memory traces are rapidly formed in the hippocampus and then replayed afterwards to allow incremental change within the neocortex. According to some, everything to be remembered is established within neocortex during this "consolidation" period (e.g., Squire \& Alvarez 1995). Others argue that only some aspects of permanent memory are established within neocortex, and that the hippocampal system is always important in retrieval of the contextual aspects of episodic memory (Moscovitch \& Nadel 1998; Nadel \& Moscovitch 1997; 1998). In the course of the replay process, the hippocampal trace is used to reactivate dispersed neocortical representations, as a consequence of which two different outcomes can occur: Categorical knowledge can be extracted from episodic experience, and associative facts can be learned. It was never clear in the McClelland et al. treatment just how these two different outcome emerge from the same reactivation mechanism.

A\&B's perspective suggests that there are two routes by which hippocampus might reactivate neocortical representations. These two routes could make quite different contributions to the reactivation process. One might suppose that reactivation by way of the temporal cortex and its representations of things in the world can lead to the formation of concepts/categories, whereas reactivation by way of the frontal cortex, and its representation of the temporal order in which things within an episode occurred, can lead to the learning of associations. This analysis maintains the separation between processes involving recognition memory (temporal neo- cortex) and episodic memory (frontal cortex), and shows how they might work together during memory consolidation. One very exciting speculation it immediately leads to is the following: What if only one part of the reactivatiion process, say the fronto-hippocampal part, is impaired? One might reactivate the various parts of an episode, but in the wrong temporal sequence. This could lead to incorrect conclusions about the causal relations inherent in prior experience. Could this be anything like what happens in schizophrenia, a disorder now known to involve damage in the fronto-hippocampal axis?

Such speculations only hint at the rich possibilities opened up by A\&B's approach. They are to be commended for their corrective action.

ACKNOWLEDGMENT

We thank Christina Charbonneau-Perry for her comments.

\section{Memory systems, frontal cortex, and the hippocampal axis}

\section{Amanda Parker \\ Department of Experimental Psychology, University of Oxford, OX1 3UD, England.amanda.parker@psy.ox.ac.uk}

Abstract: Three comments are made. The proposal that recollection and familiarity-based recognition take different thalamic routes does not fit recent experimental evidence, suggesting that mediodorsal thalamus acts in an integrative role with respect to prefrontal cortex. Second, the role of frontal cortex in episodic memory has been understated. Third, the role of the hippocampal axis is likely to be the computation and storage of ideothetic information.

Aggleton \& Brown's (A\&B's) target article presents a timely review of the substantial body of evidence that supports the circuit view of anterograde amnesia first proposed by Delay and Brion (1969). This commentary uses the target article as a starting point for further exploration of: (1) A\&B's proposed anatomical substrate for the fractionation of recognition memory, (2) the role of frontal cortex as a likely endpoint of the hippocampal axis, and (3) the ideothetic nature of the processing undertaken by the hippocampalfrontal system.

(1) A\&B propose that recollection-based recognition is processed by the hippocampal-anterior thalamic system, whereas familiarity-based recognition is the product of the perirhinalmediodorsal thalamic system. Although this is an appealing proposal, recent experimental evidence suggests that the situation is more complex than this, and that magnocellular mediodorsal thalamus (MDmc) is more likely to be acting in an integrative role with respect to the prefrontal cortex. A large impairment in recognition memory performance results from disconnection of perirhinal cortex from the mediodorsal thalamus (Parker \& Gaffan 1998a), which at first sight seems to be consistent with the proposal by Aggleton and Mishkin (1983a; 1983b) and Bachevalier et al. (1985a) that visual object recognition memory depends on information flow from temporal cortex to frontal cortex via the thalamus. However, several further considerations argue against this interpretation. First, the route of information flow from temporal cortex to thalamus as originally envisaged by these authors was via the fornix and amygdala, and we have demonstrated that with preoperative training in delayed matching-to-sample, aspiration amygdalectomy combined with fornix transection need not produce an impairment (Parker \& Gaffan 1998a). Second, as Goulet et al. (1998) recently showed, although there is a small direct projection from the perirhinal cortex to the mediodorsal thalamus, independent of synaptic relays in the hippocampus or amygdala, this direct projection passes through or very near to the amygdala and is therefore interrupted by aspiration amygdalectomy carried out in the same way. Third, bilateral lesions restricted to the medial 
portion of the magnocellular part of the mediodorsal nucleus, which is the portion that receives limbic and perirhinal afferents, had only a very mild effect on delayed matching-to-sample when compared with rhinal ablation (Parker et al. 1997). For these reasons the impairment from disconnection of perirhinal cortex from the whole of the magnocellular part of the mediodorsal nucleus cannot be explained by the direct or limbic interactions between these two structures. Instead, it seems likely that because of the extensive efferent and afferent connections of the magnocellular part of the mediodorsal nucleus with prefrontal cortex, a unilateral lesion of the whole magnocellular mediodorsal nucleus is sufficient to produce widespread dysfunction of the ipsilateral prefrontal cortex. Thus, when crossed with a unilateral perirhinal cortex ablation, a unilateral lesion of this nucleus produces a functional effect similar to the effect of a large unilateral prefrontal cortex lesion, although somewhat milder (Parker \& Gaffan 1998a). Furthermore, bilateral removal of the entire MDmc thalamus also affects object-in-place memory, suggesting that frontal cortex, and by extension MDmc, becomes particularly important when an integration of object and spatial information is necessary (Parker \& Gaffan, in press).

(2) A substantial body of the work using rats reviewed in the target article has stressed the contribution of the hippocampal axis to spatial memory. The conclusion often drawn from this evidence is that the anterior thalamic efferents to cingulate cortex and to hippocampus via the cingulum bundles complete the circuit, rather than the efferents to prefrontal cortex. As rats have little cortex that is a homologue of primate prefrontal cortex, manipulations that clarify the role of frontal cortex have not produced results that are readily interpretable. Furthermore, consideration of the effects of frontal lesions on human memory have often been inconclusive. However, we have shown in the monkey that bilateral ablations of the prefrontal cortex abolish the ability to associate even a single visual stimulus with reward (Parker \& Gaffan 1998b). This suggests that in humans with extensive bilateral frontal lesions far more severe memory impairments would be seen than those found with frontal patients of the type that have typically been studied, with much smaller and often unilateral lesions. In the monkey, the results of a series of experiments that examined the effects of lesions of fornix, mamillary bodies, and anterior thalamus on object-in-place memory have supported the proposal that the hippocampal system is preferentially important for object/spatial configural memory (Gaffan 1994b; Parker \& Gaffan 1997a; 1997b). We did not find a similar impairment after cingulate cortex ablation, however, and histological examination showed degeneration of cingulum bundles bilaterally in two cases and unilaterally in one. This led us to propose that, for object-inplace memory, connections from anterior thalamus to prefrontal cortex were critical for choosing and reaching toward a specific target on the basis of an episodic representation (Parker \& Gaffan 1997b).

(3) Recent work with both rats and monkeys suggests that the specific role of the hippocampal-anterior thalamic system in spatial memory may be in the computation and storage of ideothetic information, rather than spatial processing in more global terms, whereas extrahippocampal temporal cortex may provide a more general representation of the environment. This path integration hypothesis proposed in rats (McNaughton et al. 1996; Wishaw \& Jarrard 1996; Wishaw et al. 1997) can also be applied to monkeys (Gaffan 1998). For example, in the proposal for the object-inplace performance deficit after anterior thalamic lesion discussed above, the computation of arm movement toward the previously rewarded object on the screen is guided in part by ideothetic cues, generated by the hippocampal axis and being used by prefrontal cortices to guide voluntary movement. That prefrontal cortex and the hippocampus both play a role in the integration of movement responses to visual stimuli is also suggested by the finding that monkeys show a deficit in visuomotor conditional learning after both hippocampal and ventral frontal lesions (Murray \& Wise 1996; 1997).
Conclusions that can be drawn from the points raised in this commentary are that the hippocampal-anterior thalamic axis provides one component of memory, ideothetic information, that, together with allothetic and object information processed in other cortical regions, aids the prefrontal cortex in selecting voluntary actions on the basis of stored strategies (Parker \& Gaffan 1997c). Prefrontal cortex is particularly crucial in this process when novel items are being encoded (Parker et al. 1998), and modulation of posterior cortical areas by prefrontal cortex on the basis of this learning is likely to be via prefrontal afferents to basal forebrain, which then modulates posterior cortices via efferents through the anterior temporal stem and fornix (Gaffan et al. 1998).

\section{The neural bases of recollection and familiarity: Preliminary tests of the Aggleton-Brown model}

\author{
Alan D. Pickering \\ Department of Psychology, St. George's Hospital Medical School, University \\ of London, London SW17 ORE, England. a.pickering@sghms.ac.uk
}

Abstract: Aggleton \& Brown suggest that whereas familiarity is computed in perirhinal cortex, the hippocampus contributes to recollection. This account raises issues about the definition of amnesia, clarifies confusion about dual-process models of recognition, and sits comfortably with accounts of hippocampal function from outside the amnesia literature. The model can - and should - be tested. Some preliminary data suggest that it may need changes.

Modern behavioural neuroscience often takes an experimental measure of relevant processes from one species (e.g., the rat or monkey) and creates an analogous measure in another species (e.g., man). Parallel drug studies, or the comparison of patients with lesioned animals, may indicate that the animal preparation and measures can serve as a model of normal and abnormal human psychological processes. This cross-species approach, however, carries with it a number of dangers, including a tendency towards a "functional autonomy of methods." Simply put, the problem is that research becomes focused on the tools, assays, and indices being employed, rather than on the bigger picture (for which the tools, etc. were initially developed).

The target article by Aggleton \& Brown (A\&B) neatly illustrates how a valid question for amnesia research: "Is hippocampal damage sufficient to cause amnesia?" became transformed into: "Is hippocampal damage sufficient to impair DNMS (delayed nonmatching to sample) test performance?" They argue that answers to the latter question may be relatively uninformative in addressing the former. When the particular forced-choice format used in DNMS (and DMS) tests with animals is also used with human subjects (e.g., in the Warrington Recognition Memory Test, RMT), patients with damage restricted to the hippocampus proper show no deficits (Aggleton \& Shaw 1996).

When research methods (such as a particular behavioural test) start to become functionally autonomous there is also a danger of equating critical underlying processes directly with test performance (see also Jacoby 1991). A\&B show that relating (tests of) "recognition memory" to a particular neural substrate is an inadequate level of analysis for a proper understanding of amnesia because recognition tests are likely to involve multiple processes. Although the components of recognition that they address (familiarity and recollection) represent only a modest increase in specificity, this work is moving in the right direction.

A\&B's work raises the following issues:

1. Defining amnesia. Mayes and Downes (1997) claim "good general agreement" for a definition of amnesia in which the anterograde deficit comprises impaired recognition and recall. $A \& B$ claim that their patients with selective hippocampal damage are amnesics and yet they may show impaired recognition only under cer- 
tain circumstances. A\&B’s position seems to imply that the defining anterograde deficit in amnesia is one affecting episodic memory based on recollection. I suspect such a definitional shift will prove controversial, although it follows from the earlier comments that I applaud definitions based more on processes than on tests.

2. Testing the model. A\&B's account also has the merit of testability and refutability. For example, any patient with selective mamillary body lesions should have memory deficits similar to the selective hippocampal patients of Aggleton and Shaw. If such a patient demonstrated preserved recollection-based remembering (as crudely indexed by normal Wechsler Memory Scale scores), then A\&B's account would be invalid. Patients with damage to the recollection circuitry should show impaired recognition memory under conditions (e.g., yes-no testing) in which recollection contributes to the performance of healthy subjects (Yonelinas 1994; Yonelinas et al. 1996). Systematic testing is needed in which selective hippocampal (and other selective recollection loop damage) amnesics are evaluated in varying recognition test formats to relate their performance to the level of recollection employed by healthy controls. Finally, A\&B's model would be seriously compromised if healthy subjects did not carry out forced-choice recognition tasks (similar to the RMT) using familiarity only.

Unfortunately, the latter tests of A\&B's model rely on sound methods for estimating recollection and familiarity. A\&B themselves review some of the difficulties with the remember-know procedure and the process dissociation procedure has been widely criticised (e.g., Curran \& Hintzman 1995). Following Yonelinas, we have just completed a series of studies with healthy subjects using direct fitting of models to recognition memory receiver operating characteristics. In each of the half-dozen separate yes-no recognition studies we have carred out, the fit of a familiarity-only model was numerically superior to a dual-process model (familiarity plus recollection), in direct contrast to the results of Yonelinas et al. (1996). Moreover, neither model produced a statistically acceptable fit to the data obtained. A\&B's neat biological mapping of the dual-process accounts of recognition memory will fail if, as our data tend to indicate, normal yes-no recognition memory is not well described by dual-process models. More directly, in one pilot experiment we had healthy subjects study eight word lists, each being followed by a recognition memory test. Half of these tests (unpredictably) were yes-no, the remainder were two-alternative forced choice (2AFC) format. As already indicated, a familiarity-only model fit the yes-no data only modestly; however, the fit to the $2 \mathrm{AFC}$ data was much worse. If these early findings are reliable, then the explanation for good $2 \mathrm{AFC}$ recognition performance by selective hippocampal amnesics cannot lie in A\&B's suggestion that $2 \mathrm{AFC}$ tests can be performed accurately using familiarity information only.

3. The relationship between familiarity and priming. One further problem with dual-process accounts of recognition is that some of their proponents (e.g., Jacoby 1991) have claimed that familiarity in recognition equates with perceptual fluency/implicit memory. As $\mathrm{A} \& \mathrm{~B}$ point out (sect. 6) this seems wrong phenomenologically (as shown by the alternative description of familiarity as a "know" state). Although they stop short of strong statements, A\&B's model clearly separates familiarity (perirhinal cortex) from priming (which involves posterior cortical regions). Objections to dual-process accounts of recognition based on contradictory evidence from experiments manipulating fluency (see Squire 1992, pp. 205-207) are neatly resolved by A\&B's framework.

4. Links to other theories of hippocampal function. As noted previously (Pickering 1997) it seems unparsimonious that the hippocampus is widely acknowledged to have other functions outside the memory domain. In fact, the hippocampus has been described as part of a system of associative prediction and hence mismatch detection (Gray 1982). A\&B cite direct evidence for this in the work of Honey et al. (1998), and the involvement of the hippocampus specifically in recollection, but not familiarity, is consistent with these more general formulations. Neural network modelling by Levy (e.g., Prepiscus \& Levy 1994) has shown that the architecture of the hippocampus is suited to learning (long) chains of associations between items in a way that could support both recollection and mismatch detection. A\&B's model should prompt a search for a detailed understanding of the processes supporting recollection and familiarity.

\section{Mere functional characterization is not enough to understand memory circuits}

\section{Alessandro Treves \\ Programme in Neuroscience, SISSA, 34014 Trieste, Italy. ale@limbo.sissa.it www.sissa.it/}

Abstract: What exactly is going on via fornical connections? Aggleton \& Brown's target article correctly stresses their importance, but a detailed understanding of their role in memory appears to require fresh research approaches.

After having eaten with Malcolm Brown in a Kyoto restaurant in 1995, I was trying to remember whether he had spelled out his anterior thalamic view.

I turned to my hippocampus for help: "Can you recall the scene?"

Hipp: "Sure, he was wearing that nice green jacket."

"Do you remember what he was saying?"

Hipp: "Yes. He was trying to get that ever-smiling waiter to understand that you would not eat any meat."

"But was he emphasizing your interaction with the mamillary bodies?"

Hipp: "Interaction? What does that mean?"

"It means, what do you do with the mamillary bodies?"

Hipp: "Oh, well, we talk to each other."

"You mean, same as with the entorhinal cortex?"

Hipp: "Sure, with entorhinal too, we just talk!"

Aggleton \& Brown's (A\&B's) is a very convincing and useful view based largely on neuropsychological evidence. It is helpful to point out the sometimes neglected connections via the fornix, which are certainly there to play some important role; at the very least, they allow the structures at the two ends of the line to "just talk." I am not sure, though, that conventional neuropsychological analyses of humans or animals, examining qualitative deficits in behaviour, can go beyond an initial clarification of the sort of messages that are exchanged along that line, and why they need to be exchanged.

A very important approach to understanding memory operations, if it were possible, would ideally be to wiretap all conversations through the fornix, and analyze them with date, sender, receiver and an interpreter to translate them from neurospeak. Neurophysiological research has made some progress in this direction (Rolls et al. 1998), exploring the information content of neuronal messages from these parts of the brain not only qualitatively but quantitatively. One perspective afforded by hippocampal recordings so far is the relative increase in the heterogeneity of significant correlates of neuronal activity from rodents (Gothard et al. 1996; O'Keefe \& Burgess 1996) to primates (Eifuku et al. 1995; Miyashita et al. 1989; Rolls \& O’Mara 1995; Rolls et al. 1993). If we were able to record from human hippocampal neurons, not at the gross scale allowed by PET and fMRI but individually, and not sporadically but systematically, we might expect even wider heterogeneity of neuronal concerns (Mirsky et al. 1997). It is difficult to believe that such variety (yet to be demonstrated, I admit) could be encapsulated into simple, clear-cut categories such as recognition and recall. A differential involvement of distinct units and connections in operations that "tax primarily familiarity judgements" and in those that "retrieve episodic items" is quite conceivable, and the evidence reviewed is convincing. However, to go from this to the notion of two parallel systems, one designed for recognition and one for recall, one must jump quite a conceptual distance. 
Analyses of the requirements for internal consistency of the relevant network operations, such as those we have proposed (Treves et al. 1996b) are limited in that they do not relate directly to human clinical categories, but this limitation can also be considered a virtue in that they invoke only intrinsic as opposed to anthropomorphic information processing notions. Such analyses have, for example, indicated the importance of quantitative variations in information content-related parameters in determining the performance of a memory system. Thus, the differential implication of distinct circuits in recognition and recall tasks are more likely to be one of the consequences of quantitative differences in, for example, activation sparsity or connectivity parameters, than the outcome of evolutionary pressure to develop qualitatively separate systems for recognition and for recall.

Beyond a qualitative description of the correlates of neural activity, single and multiple single unit recording likewise yields access to the structure of neuronal representations, bringing out for example quantitative differences in the way the same spatial correlates are encoded by neighboring populations (Treves et al. 1996a; 1998). These structural differences may also be revealed by nonconventional use of neuropsychological tests, such as examining the distribution of performance errors by memory patients of different categories in a Famous Faces test (Lauro-Grotto et al. 1997). The activation and connectivity patterns that underlie such differences probably satisfy internal consistency requirements in the organization of the networks involved (Fulvi-Mari \& Treves 1998).

In conclusion, reviews like A\&B's illustrate the need to integrate more traditional approaches to memory research, those reflecting the point of view of the subject, with all the approaches now available, examining memory transactions from the point of view of the actors - the neurons. Such contamination has in my view helped assess the perforant path, and it should prove no less fertile once fornically extended.

\section{The hippocampus and path integration}

\section{Ian Q. Whishaw \\ Department of Psychology and Neuroscience, University of Lethbridge, Lethbridge, Alberta, Canada, T1K 3M4.whishaw@uleth.ca}

\begin{abstract}
Recent studies of the contribution made by the hippocampus to spatial behavior suggest that it plays a role in integrating and double integrating distance and direction information using cues generated by selfmovement. This and other evidence that the hippocampus plays a central role in spatial behavior seems inconsistent with proposals that it is primarily involved in episodic memory.
\end{abstract}

It is well known that damage to the hippocampus and/or fimbria fornix impairs the ability of rats to learn to swim to a hidden platform (place) in a swimming pool but not their ability to learn to swim to a visible platform (cue). The place/cue dissociation is central to theories that suggest a special spatial role for the hippocampus. Aggleton \& Brown (A\&B) however, propose that the dissociation supports their contention that the extended hippocampus plays a special role in episodic memory (sect. 5.1). We question whether this claim advances our understanding of the function of the hippocampus.

We have been investigating an important feature of the place deficit in hippocampal rats. Such rats, after training to swim to a visible cue in the swimming pool, will swim directly to the cue's previous location and search that location after the cue is removed. Four aspects of their performance suggest that they have learned a place response as accurately as do control rats, even though the demands of the task did not require that they do so: (1) They swim accurately from start points located at different distances and in different directions from the correct location (Whishaw \& Jarrard 1995; 1996; Whishaw et al. 1995); (2) they search in the correct location indicating that they expect to find the platform there; (3) they make an accurate swim from a novel location (Whishaw 1998); and (4) they are more persistent than control rats in returning to that location (Whishaw \& Tomie 1997b). Cuing the location of the platform does not simply allow the hippocampal damaged animals to overcome a problem of slow learning because if cued and hidden place trials are intermixed, hippocampal acquisition matches that of control rats (Whishaw \& Tomie 1997a). Nor is the learning nonvisual, because if the rats are blindfolded, they, like control rats, are impaired (Whishaw 1998). These results indicate that the place deficit in hippocampal rats is not in the episodic domain of "knowing where" but in the spatial domain of "getting there." Independent studies using a shrinking hidden platform provide support for the generality of this conclusion (Day \& Schallert 1996).

We propose that hippocampal rats have an impairment in using self-movement cues (idiothetic cues) and path integration (Whishaw et al. 1997). To know where they came from, animals can make a record of their own movements and then integrate that record to link the starting point to the goal. On a subsequent trial, they can double-integrate the record to generate a more direct response. This strategy of navigation is referred to as dead reckoning or sense of direction (Barlow 1964). To investigate the role of the hippocampus in path integration, we designed a task in which a rat leaves a hidden burrow to forage for a piece of food placed at an indeterminate location. The piece of food is large enough that when found, it is carried back to the burrow for consumption. Once the rats find and carry food to a home base, they are blindfolded and started from a novel location. Control rats return accurately to the new starting location, whereas hippocampal rats do not (Whishaw \& Maaswinkel 1998).

Information obtained from path integration can be used not only for dead reckoning, but also to create a spatial frame of reference and to calibrate the distances and directions between objects. Accordingly, information from path integration can be used to solve laboratory problems such as spatial reversals presented to rats by A\&B. When spatial cues that once signaled a place suddenly become meaningless, the animal can maintain its spatial orientation using self-movement cues until it discovers which distal cues signal the problem's new solution (Whishaw \& Tomie, 1997a).

Our conclusion that the hippocampal formation has a fundamental role in spatial behavior, perhaps even in a single computational form of spatial behavior, is consistent with a growing literature inspired by the spatial hypothesis (O'Keefe \& Nadel 1978). We argue that the hippocampal rat's episodic abilities combined with the poverty of the explanation for making predictions limits the episodic theory's utility. In other words, the hazard in arguing that brain regions play fundamental mnemonic roles either independently or as systems, detracts from the goal of explaining how those same brain regions contribute to adaptive behavior.

\section{The medial dorsal nucleus of the thalamus is not part of a hippocampal-thalamic memory system}

\author{
Menno P. Witter and Ysbrand D. Van der Werf \\ Graduate School of Neurosciences Amsterdam, Research Institute- \\ Neurosciences, Vrije University, Department of Anatomy and Embryology, \\ 1081 BT Amsterdam, The Netherlands. \\ \{mp.witter.anat; yd.van_der_werf.anat\}@med.vu.nl
}

Abstract: Aggleton \& Brown propose that familiarity-based recognition depends on a perirhinal-medial dorsal thalamic system. However, connections between these structures are sparse or absent. In contrast, the perirhinal cortex is connected to midline/intralaminar nuclei. In a human, a lesion in this thalamic domain, sparing the medial dorsal nucleus, impaired familiarity-based recognition while sparing recollective-based recognition. It is thus more likely that the intralaminar/midline nuclei are involved in recognition. 
Aggleton \& Brown (A\&B) are to be complimented for their important conceptual contribution about memory formation and retrieval. Their arguments for a dissociation between recollective- and familiarity-based recognition are impressive. Their claim that for the former the hippocampal-fornix-anterior thalamic system is of vital importance accords well with our conclusions based on the neuropsychological assessment of a recently collected series of patients with restricted thalamic infarctions, taken in conjunction with a recent meta-analysis of the literature (van der Werf et al., unpublished observations). We concluded that the fibre tract connecting the mammillary bodies with the anterior nuclei of the thalamus (the mammillo-thalamic tract) is necessarily affected in those subjects where damage to the thalamus is associated with an amnesic syndrome (cf. Graff-Radford et al. 1990; Von Cramon et al. 1985).

In contrast, we doubt whether A\&B's second proposition, that familiarity-based recognition is mediated by a perirhinal-medial dorsal system, stands scrutiny. Based on two lines of evidence, we particularly question the participation of the medial dorsal nucleus of the thalamus in this recognition system. First, monosynaptic projections from perirhinal cortex to the medial dorsal nucleus appear either sparse or nonexistent in animal species. Although the authors refer to two tracing reports in the monkey (sect. 7, para. 2; Aggleton et al. 1986a; Russchen et al. 1987), from those papers it is clear that perirhinal-medial dorsal projections are sparse. More detailed studies in the rat (Burwell et al. 1994; Chen \& Burwell 1996; our own unpublished observations) and the cat (Room \& Groenewegen 1986; Witter \& Groenewegen 1986) indicate that the perirhinal cortex projects massively to the posterior complex of the thalamus and to certain components of the intralaminar/midline thalamic nuclei, including the reuniens and central medial nuclei. In the monkey, a comparable projection pattern prevails (Suzuki, personal communication).

Second, we have recently described a patient suffering from cognitive deficits after a thalamic infarction (Van der Werf et al. 1998). This patient shows a dissociation between the two types of recognition described in the target article. It is interesting, however, that this dissociation takes the form of a sparing of recollective-based recognition with a deficit in familiarity-based recognition. This is evident from normal scores on the Visual Association Learning Task (Lindeboom 1989; Rombouts et al. 1997), a test probing the recall of visual associations. On the other hand, results on the classical Recognition Memory Test for faces (Warrington 1984) are deficient. If one takes the viewpoint of $A \& B$ (sect. 6 , para. 7), the latter finding indicates an impaired familiarity-based recognition. The lesion in this patient was shown to spare the medial dorsal and the anterior nuclei as well as the mammillothalamic tract, but to involve the region of the right lateral internal medullary lamina (IML). In contrast, patients with similarly sized lesions just outside the lateral IML, involving the right medial dorsal nucleus, do not show an impairment of familiarity-based recognition (Kritchevsky et al. 1987; Shuren et al. 1997). This observation corroborates our conclusion that the IML and/or nuclei within it, rather than the medial dorsal nucleus, is involved in this type of recognition. It is of interest that SPECT (single photon emission computer tomography) imaging and extensive neuropsychological testing of our patient led us to conclude that this patient had a dysfunction of "executive" or prefrontal" cognitive processes rather than hippocampal, formation-dependent processes. This combination of familiarity-based recognition and prefrontal symptoms is in line with the account given in section 7 , paragraphs $2-4$.

That the perirhinal cortex may contribute in a unique way to the functioning of the medial temporal lobe memory system, as proposed earlier by Eichenbaum et al. (1994), is in line with our recent electrophysiological and anatomical findings that the perirhinal cortex has direct reciprocal connections to CA1 and the subiculum. Those connections show a topographical organization that differentiates them from the more widespread projections that originate from the entorhinal cortex. Based on these observations, we proposed that the perirhinal inputs to the hippocam- pal formation may be crucially involved in ongoing comparisons of newly acquired information as carried out by the hippocampal system (Naber et al., submitted). As such, this proposition would be complementary to a familiarity-based recognition process as proposed by $\mathrm{A} \& \mathrm{~B}$. Whether the perirhinal cortex has specific functional relations with thalamic domains, different from those associated with the hippocampal system, remains to be established. If so, likely candidates are the midline/intralaminar nuclei (see also sect. 5.1, para. 6), instead of the medial dorsal nucleus.

\section{ACKNOWLEDGMENTS}

This research has been supported by grants 903-47-008 and 970-10-012 of the Netherlands Organization for Scientific Research (NWO).

\section{The neural substrates of recollection and familiarity}

\author{
Andrew P. Yonelinas a, Neal E. A. Krolla, Ian G. Dobbins ${ }^{a}$, \\ Michele Lazzara ${ }^{a}$, and Robert T. Knight ${ }^{\mathrm{b}}$ \\ a Department of Psychology, University of California at Davis, Davis, CA \\ 95616; 'b Department of Psychology, University of California at Berkeley, \\ Berkeley, CA 94720. \\ \{apyonelinas; neakroll; igdobbins; mmlazzara\}@ucdavis.edu \\ rtknight@socrates.berkeley.edu
}

\begin{abstract}
Aggleton \& Brown argue that a hippocampal-anterior thalamic system supports the "recollection" of contextual information about previous events, and that a separate perirhinal-medial dorsal thalamic system supports detection of stimulus "familiarity." Although there is a growing body of human literature that is in agreement with these claims, when recollection and familiarity have been examined in amnesics using the process dissociation or the remember/know procedures, the results do not seem to provide consistent support. We reexamine these studies and describe the results of an additional experiment using a receiver operating characteristic (ROC) technique. The results of the reanalysis and the ROC experiment are consistent with Aggleton \& Brown's proposal. Patients with damage to both regions exhibit severe deficits in recollection and smaller, but consistent, deficits in familiarity.
\end{abstract}

Aggleton \& Brown (A\&B) argue that a hippocampal-anterior thalamic system supports the "recollection" of contextual information about previous events, and that a separate perirhinal-medial dorsal thalamic system supports detection of stimulus "familiarity." There is a growing body of human literature that is consistent with these claims, showing that recognition memory judgments reflect the separate contributions of recollection and familiarity processes. These two processes are functionally independent (e.g., Atkinson \& Juola 1974; Jacoby 1991; Mandler 1980) and they exhibit separate electrophysiological correlates (e.g., Düzel et al. 1997), suggesting that they reflect partially distinct cortical generators. Some studies of human amnesia support A\&B’s proposed mapping of these processes to areas within the medial temporal lobes. Other recent reports appear to be in conflict, however. Reexamining the human literature on recollection and familiarity in amnesia finds that the current evidence is, in fact, quite consistent with their proposal.

According to A\&B's proposal, patients with damage to both the hippocampal and perirhinal systems - which includes most of the human amnesic patients that have been studied - should exhibit deficits in both recollection and familiarity. Direct tests of recognition memory provide some support for this prediction in showing that amnesics typically exhibit recognition memory deficits. However, because recognition memory judgments can be based on either recollection or familiarity, these results do not show if the deficits are in both recollection and familiarity, or restricted to a single process. Therefore, it is necessary to look to procedures that provide separate measures of these two processes.

$A \& B$ describe a study by Knowlton and Squire (1995) using the remember/know procedure (Tulving 1985a) that found amnesics 
were less likely to respond that they "knew" on the basis of familiarity that test items were studied, indicating that familiarity was disrupted. However, A\&B also describe a study by Verfaellie and Treadwell (1993) using the process dissociation procedure (Jacoby 1991), that concluded that familiarity was preserved in amnesia. More recent studies using the remember/know procedure have led to different conclusions than either of the earlier studies. For example, Schacter et al. (1996) found that the proportion of knowing responses actually increased significantly for amnesic patients relative to healthy controls, indicating that amnesia was associated with an increase in familiarity. Given that all three of these studies included patients with widespread damage to the medial temporal lobes, if $A \& B$ are right, these patients should have exhibited deficits in both recollection and familiarity. On the surface, therefore, human amnesia data does not appear to provide consistent support for their new proposal.

However, a closer examination of these studies finds that the previous interpretations of these results were incomplete in that they did not fully account for response biases or, in the case of the remember/know studies, did not correct their measures to account for the mutual exclusivity between the remember/know responses. That is, in all of the reported studies the amnesics exhibited higher false alarm rates than the controls and this biased the estimates of recollection and familiarity. Moreover, the probability of a "know" response in the remember/know procedure is mathematically constrained by the proportion of remember responses, so that "knowing" responses by themselves do not provide an accurate measure of familiarity. In a recent article (Yonelinas et al. 1998), we reanalyzed the results of these earlier studies using a dual-process signal-detection model (Yonelinas 1994) that allowed for the independent contribution of recollection and familiarity and incorporated signal detection theory to accommodate differences in false alarms. We also tested additional amnesics using a receiver operating characteristics (ROC) analysis to validate the model and to derive estimates of recollection and familiarity in these patients.

The ROC analysis showed that the model provided an accurate account of recognition performance. Most important, however, was that all three estimation procedures converged in showing that both recollection and familiarity were disrupted in amnesics. Figure 1 summarizes the results of the remember/know, process dissociation, and ROC experiments. The results provide consis- tent support for A\&B's proposal, in that patients with damage to both the hippocampal and perirhinal systems exhibit the expected deficits in both recollection and familiarity.

The results are also consistent with previous studies showing that amnesia is associated with a disproportionate disruption of recollection compared to familiarity (e.g., Kroll et al. 1996). Although familiarity was consistently disrupted in the amnesics, recollection was disrupted to a much greater extent. This disproportional deficit was observed in every condition of every experiment we examined. Note that recollection and familiarity are measured on different scales in Figure 1. The disproportional deficit in recollection is also observed, however, when the familiarity $d$ ' scores are converted to probabilities.

These results join a growing body of studies that show recollection and familiarity to be functionally dissociable memory processes, and suggest that they rely on distinct anatomical regions. However, a critical test of A\&B's specific proposal will be to determine whether patients with damage that is restricted to the hippocampal system or to the perirhinal system will exhibit selective deficits of recollection and familiarity.

\section{Remembering the hippocampus}

\author{
Stuart M. Zola ${ }^{a, b}$ and Larry R. Squire ${ }^{a, b, c}$ \\ a Veterans Affairs Medical Center, San Diego, CA 92161; ${ }^{\mathrm{b}}$ Departments \\ of Psychiatry and Neurosciences, University of California, La Jolla, CA \\ 92093; ' Department of Psychology, University of California, La Jolla, CA \\ 92093. \{szola; Isquire\}@ucsd.edu
}

\begin{abstract}
The proposal that the hippocampus is important for the encoding of episodic information, but not familiarity-based recognition, is incompatible with the available data. An alternative way to think about functional specialization within the medial temporal lobe memory system is suggested, based on neuroanatomy.
\end{abstract}

Damage to the hippocampus and adjacent, anatomically related structures impairs memory in rats, monkeys, and humans (Bunsey \& Eichenbaum 1996; Mishkin \& Murray 1994; Squire 1992; Squire \& Zola-Morgan 1991). In both humans and monkeys, it is established that memory impairment becomes more severe as
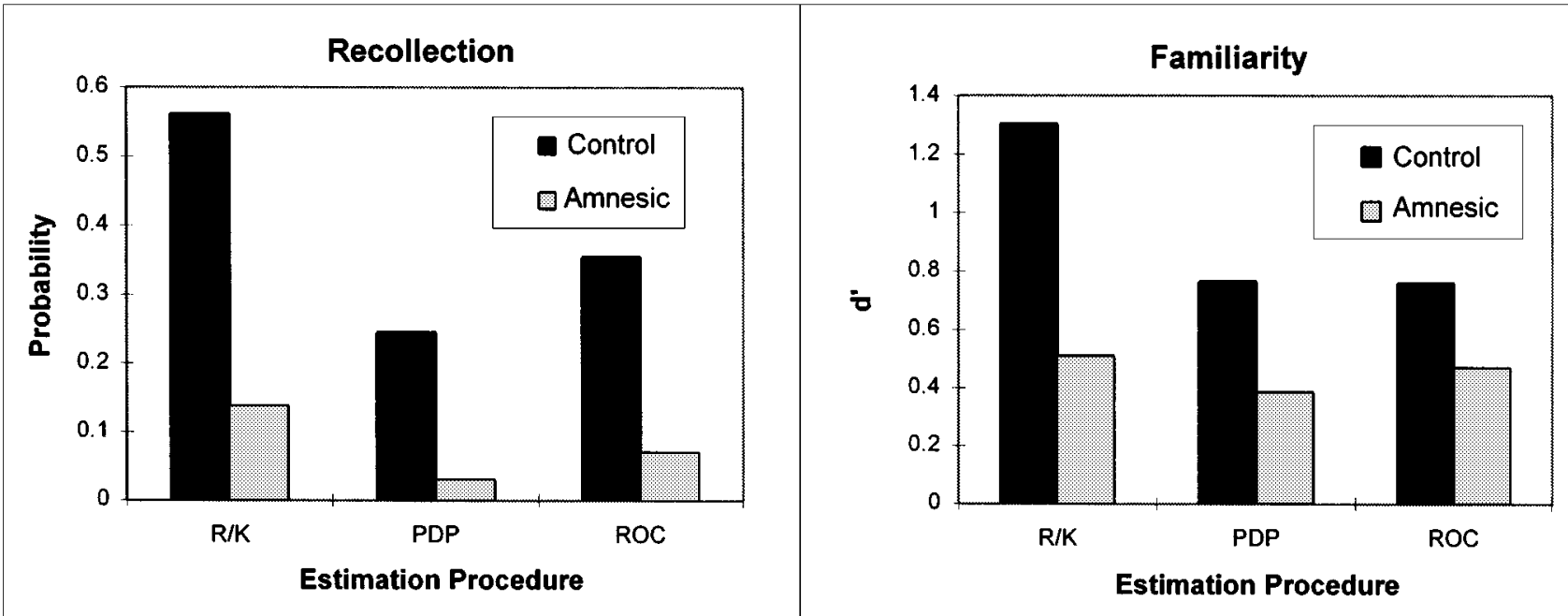

Figure 1 (Yonelinas et al.). Estimates of recollection and familiarity in amnesics and controls derived using the remember/know (R/ $\mathrm{K}$ ), the process dissociation (PDP), and the receiver operating characteristic (ROC) procedures. The R/K data reflect the average estimates derived from Knowlton and Squire (1995), Schacter et al. (1996 and 1997); the PDP data are from Verfaellie and Treadwell (1993); and the ROC data are from Yonelinas et al. (1998). All three procedures showed that amnesics exhibited a reduction in recollection and a smaller but consistent reduction in familiarity. 
more of these structures are damaged (Rempel-Clower et al. 1996; Zola-Morgan et al. 1994). As we have pointed out previously (Zola-Morgan et al. 1994), this fact is consistent with the possibility that the different structures make different contributions to memory functions. The target article by Aggleton \& Brown (A\&B) is the most recent of several efforts to consider what these different contributions might be (Eichenbaum et al. 1994; Gaffan 1994a; Vargha-Khadem et al. 1997). A\&B propose that the hippocampus itself (and its diencephalic targets) is important for the encoding of episodic information. By contrast, the judgment of familiarity (simple recognition memory) does not require the hippocampus, but depends instead on the perirhinal cortex (and its diencephalic targets).

The attempt to find functional specialization within the medial temporal lobe is worthwhile, but the view proposed by A\&B encounters a number of problems. First, they view the distinction between episodic and semantic memory (Tulving 1991) and the distinction between remembering and knowing (Tulving 1985b) as closely related to their own. These distinctions have been useful and important for separating medial temporal lobe function from frontal lobe function, but it is not at all clear that they partition medial temporal lobe function itself (Knowlton \& Squire 1995; Squire \& Zola 1991). Second, the specific distinction proposed by $\mathrm{A} \& \mathrm{~B}$ (episodic memory versus recognition memory) is not supported by the data. Here we focus on A\&B's view that recognition memory does not require the integrity of the hippocampus. We first mention findings from humans and rats and then consider in more detail the findings from monkeys.

First, amnesic patients R. B. and G. D., who had lesions restricted to the CA1 region of the hippocampus, were impaired at recognition memory (Reed \& Squire 1997; Zola-Morgan et al. 1986). In patients with damage involving all the cell fields of the hippocampus and some cell loss in entorhinal cortex (patients L. M. and W. H., Rempel-Clower et al. 1996), recognition memory was also unmistakably impaired (Reed \& Squire 1997). Second, rats with hippocampal lesions were impaired on the delayed nonmatching-tosample task after a 2-minute delay (Mumby et al. 1995).

Third, the weight of evidence is that monkeys with hippocampal lesions have impaired recognition memory. There have been seven studies involving lesions limited to the hippocampus that assessed performance on either the delayed nonmatching-to-sample task or the visual paired-comparison task. The lesions were produced by ischemia, radio-frequency, or ibotenic acid. Four of the studies are published or in press (Alvarez et al. 1995; BeasonHeld et al. 1998; Murray \& Mishkin 1998; Zola-Morgan et al. 1993), and three are preliminary reports published in Abstract form (Clark et al. 1996; Teng et al. 1998; Zola et al. 1998). Of these, only one study (Murray \& Mishkin 1998) found no deficit. This study was the only one that used two-stage surgery and the only one in which the nonmatching rule was trained preoperatively (for discussion, see Zola \& Squire 1998).

Recent results from our laboratory (Fig. 1) show that, as measured by the delayed nonmatching-to-sample task, hippocampal lesions made by ischemia, radio-frequency, or ibotenic acid impair recognition memory in monkeys to a similar degree (Zola et al., in press). For all three groups, surgery was done in a single stage, and the nonmatching rule was trained postoperatively. The deficit was present whether animals were removed from the test apparatus during the delay intervals or allowed to remain in the apparatus (Teng et al. 1998).

Questions have been raised as to whether ischemic lesions can provide a valid method for assessing hippocampal function (A\&B; Bachevalier \& Meunier 1996; Nunn \& Hodges 1994). If ischemia caused cell loss in the hippocampus but also caused neuronal dysfunction (not cell death) in other structures important for memory, then the hippocampal cell loss that is detected in histological exams need not be the cause of memory impairment. This issue can be approached experimentally by comparing the effects on memory of ischemic lesions and similarly-sized stereotaxic lesions within the hippocampal region.

When this was done, the effects of ischemia (Zola-Morgan et al. 1992) closely approximated the effects of radio-frequency lesions (Alvarez et al. 1995; also see Squire \& Zola-Morgan 1996) (Fig. 1). $A \& B$ point to a trend in our data, whereby ischemic animals performed worse across the delays of the performance test than the animals with radio-frequency lesions $(79.3 \%$ correct vs. $84.6 \%$ correct). At the longest delay at which both groups were tested (10 min), the corresponding scores were $68.1 \%$ correct and $68.5 \%$ correct, respectively $(p>1.0)$. We do not know how to evaluate small numerical differences that do not approach statistical significance. In any case, the nature of ischemic lesions could be fruitfully pursued in the rat. What is needed are new parametric studies that assess memory performance as a function of the size of stereotaxic lesions (for this approach, see Moser et al. 1993; 1995). One could then ask whether ischemic lesions of varying size affect performance to the same extent as similarly sized stereotaxic lesions, or whether the impairment following ischemia is more severe.

A consideration of functional specialization within the medial temporal lobe should be guided by the neuroanatomy of the region. The medial temporal lobe region is a convergence zone for unimodal and polymodal inputs from neocortical association ar-
ISCHEMIC (ISC)

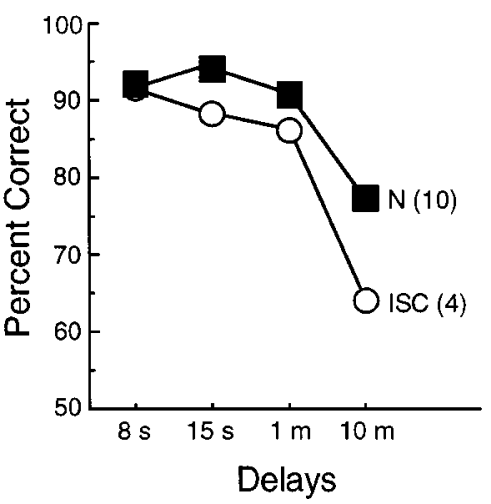

RADIO FREQUENCY (RF)

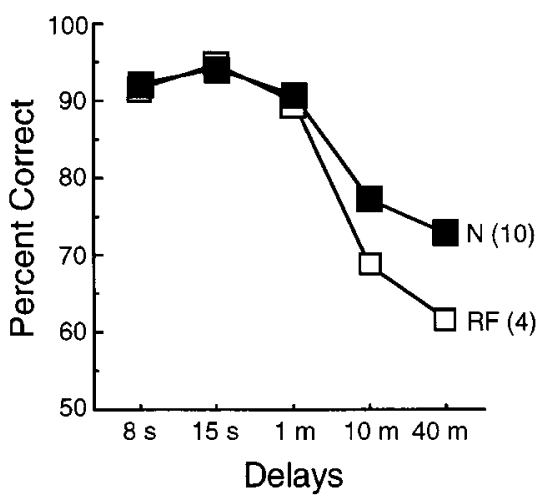

IBOTENIC ACID (IBO)

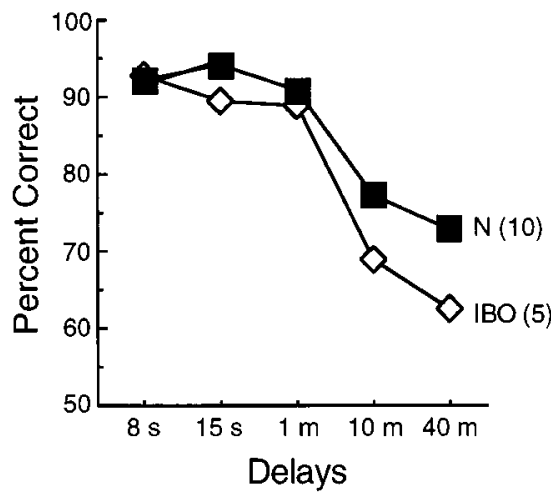

Figure 1 (Zola \& Squire). Monkeys with lesions limited to the hippocampal region (the dentate gyrus, the cell fields of the hippocampus, and the subiculum) made by ischemia (ISC, $\mathrm{n}=4$ ), radio-frequency $(\mathrm{RF}, \mathrm{n}=4$ ), or ibotenic acid $(\mathrm{IBO}, \mathrm{n}=5)$ were similarly impaired on the delayed nonmatching-to-sample task. When the scores at all delays $\geq 15 \mathrm{sec}$ were combined, each lesion group was impaired ( $\mathrm{N}=10$ normal control monkeys; all $p \mathrm{~s}<0.05)$. (From Zola \& Squire, in press). 
eas, areas important for information processing and ultimately for long-term memory storage. The first clue to how the components of the medial temporal lobe function comes from the fact that different parts of the neocortex access the medial temporal lobe at different points (Suzuki \& Amaral 1994a). For example, the higher visual areas TE and TEO project preferentially to the perirhinal cortex. Conversely, input about spatial information that comes to the medial temporal lobe from parietal cortex arrives exclusively at the parahippocampal cortex. With this anatomy in mind, one might expect perirhinal cortex to be especially important for visual memory and the parahippocampal cortex to be important for spatial memory. Findings to date are consistent with this expectation (Malkova \& Mishkin 1997; Parkinson et al. 1988; Ramus et al. 1994; Teng et al. 1997).

The hippocampus itself is the final stage of convergence within the medial temporal lobe, receiving input from both the perirhinal and parahippocampal cortices, as well as from the entorhinal cortex. The entorhinal cortex receives about two-thirds of its cortical input from the parahippocampal and perirhinal cortices. Reasoning again from anatomy, one might expect the hippocampus to be involved in both visual and spatial memory, combining all the operations of memory formation that are carried out independently by the more specialized structures that project to it. Again, the data seem to bear this out. In patients with damage restricted to the CA1 region of hippocampus, the memory impairment extends across all sensory modalities and types of material. The memory impairment is only modest because considerable memory function can be supported by the cortical components of the medial temporal lobe system.

There has been an eagerness to identify a separate and distinct subfunction for the hippocampus itself. If there is some independent function that is not shared at all by adjacent cortex, then for some memory operation the CAl patient should exhibit a deficit as severe as that observed in a patient like H. M., whose lesion includes the hippocampus as well as most of the medial temporal lobe. Yet the CA1 patient presents as a mild version of $\mathrm{H}$. M. Indeed, regardless of how memory is measured, the impairment is less severe following a small lesion within the hippocampus (amnesic patients R. B. and G. D.) as compared to a large medial temporal lobe lesion (patient H. M.). A\&B's proposal provides no account of this simple fact.

Finally, it is unclear to us why evidence that hippocampal lesions do impair recognition memory has sometimes been considered unwelcome on theoretical grounds. Even a recognition (or familiarity) decision requires that a link be made between the stimulus presented in the retention test and what was presented during learning. The recognition test does not ask the subject whether an item has ever been seen or whether one knows what an item is. It asks whether an item that appeared recently is familiar. It is this association, this relational work, that many have supposed is at the heart of declarative memory and hippocampal function in both humans and nonhuman animals (Eichenbaum 1997; Squire 1992; Sutherland \& Rudy 1989).

\section{Authors' Response}

\section{Thanks for the memories: Extending the hippocampal-diencephalic mnemonic system}

\author{
John P. Aggleton a and Malcolm W. Brown ${ }^{\mathrm{b}}$ \\ a School of Psychology, Cardiff University, Cardiff, CF1 3YG, Wales; \\ ${ }^{\mathrm{b}}$ Department of Anatomy, University of Bristol, Bristol, BS8 1TD, England. \\ aggleton@cardiff.ac.uk www.cf.ac.uk/uwc/psych/ \\ m.w.brown@bristol.ac.uk www.bris.ac.uk/depts/anatomy
}

Abstract: The goal of our target article was to review a number of emerging facts about the effects of limbic damage on memory in humans and animals, and about divisions within recognition memory in humans. We then argued that this information can be synthesized to produce a new view of the substrates of episodic memory. The key pathway in this system is from the hippocampus to the anterior thalamic nuclei. There seems to be a general agreement that the importance of this pathway has previously been underestimated and that it warrants further study. At the same time, a number of key questions remain. These concern the relationship of this system to another temporal-lobe/diencephalic system that contributes to recognition, and the relationship of these systems to prefrontal cortex activity.

One of the purposes of a $B B S$ target article is to elicit debate by raising a potentially contentious viewpoint and then seeing how it stands up under the scrutiny of expert commentary. After the dust has settled it is easier to determine whether the consensus view in the field has altered and where the key areas of debate are. We feel that the insightful commentaries contributed have helped to achieve both goals. Thus it seems apparent that there has been a shift in our understanding of the neuroanatomical basis of the amnesias, leading to a reappraisal of what that information can tell us about neural systems underlying aspects of memory (Nadel et al.). This is most evident in the general acceptance of the importance of the direct outputs from the hippocampus to the rostral diencephalon, and the associated loss of the division between temporal lobe and diencephalic amnesias. At the same time, there is much uncertainty over the relationships of these pathways to cortical areas in the temporal and frontal lobes. To address these issues it is perhaps simplest to restate the "main features of the proposed model" and to relate these to the various commentaries.

\section{R1. The hippocampal efferents via the fornix to the mamillary bodies and medial thalamus, most especially the anterior nuclei, are vital for normal hippocampal activity}

The fornix contains a variety of pathways that project both to and from the hippocampus. That cutting the fornix often mimics the effects of hippocampectomy is obviously a consequence of this fact. Less straightforward has been the identification of those pathways that are most critical, and whether they are afferent or efferent to the hippocampus. The target article sought to emphasize the importance of the hippocampal projections to the anterior thalamic nuclei, both those that are direct and those that are indirect via the mamillary bodies. This emphasis was approved either explicitly (Burwell \& Eichenbaum, DalyrympleAlford et al., Foster, Gabriel \& Smith) or tacitly by the 
large majority of commentators. This is noteworthy as it marks a clear shift away from the influential notion of separate temporal lobe and diencephalic systems in normal episodic memory.

Knowlton made the point that our model overemphasized the hippocampal/diencephalic connections to the detriment of those between the hippocampus and the prefrontal cortex. Although we did acknowledge the likely importance of these prefrontal connections (see sect. R9), they were not included in Figure 1, as this would have distracted from the chief purpose of the article, which was to consider the hippocampal/anterior-thalamic axis. Moreover, a recent series of disconnection studies in our laboratory (Warburton et al., unpublished findings) has further emphasized the importance of these hippocampal-thalamic connections. We examined the effects on rat behaviour of crossed unilateral lesions involving the fornix in one hemisphere and the anterior thalamic nuclei in the other hemisphere. Such lesions, which disconnect the hippocampus from the anterior thalamic nuclei, led to clear deficits on tests of spatial working memory. This was not found for unilateral lesions in the same hemisphere. It is interesting that this disconnection effect was observed only when the fornix lesion included the hippocampal commissure (Olton et al. 1982). We have also examined crossed lesions between the hippocampus and the anterior thalamic nuclei and found similar results. These results do not show that other fornical connections (such as those with the basal forebrain and septum) are not important, but they do strongly indicate that the similarities between the spatial memory deficits observed after anterior thalamic lesions and hippocampal lesions are more than just superficial.

Krieckhaus has discussed a possible anomaly with the model whereby mamillary body lesions could lead to a greater deficit than fornix lesions. He offers an ingenious account to explain how this could occur, but we are unaware of relevant examples of animal studies where this unusual pattern of results is found. Nevertheless, anterior thalamic damage that involves the lateral dorsal nucleus could be more disruptive than fornix damage as this nucleus receives nonfornical inputs from the subiculum (Aggleton \& Saunders 1997; Warburton \& Aggleton 1999; Warburton et al. 1997). Although earlier clinical reviews indicated that fornix damage might have little or no effect on memory (e.g., Garcia-Bengochea \& Friedman 1987), subsequent clinical studies have consistently linked fornix damage with memory loss (see sect. R3).

\section{R2. This extended hippocampal-diencephalic system becomes more diffuse beyond the anterior thalamic nuclei, but includes two major components. Whereas one component returns to the temporal lobe, principally via the cingulum bundle, the other projects to prefrontal and cingulate cortices}

Little was said about these proposals, presumably because they are largely drawn from established anatomical knowledge. Parker did, however, report a lesion study in which cingulum bundle damage was without effect, even though the task is sensitive to hippocampal/fornix/anterior-thalamic damage. This is potentially important and may point to the fact that partial damage to these more diffuse, multiple pathways is unlikely to produce the full behavioural deficit. Surprisingly little is known about the functions of the cingulum bundle and it is hoped that these proposals will help draw attention to this structure.

\section{R3. This extended hippocampal-diencephalic system is critical for episodic memory and damage to the component structures can result in similar memory impairments}

The closer linking of temporal lobe and diencephalic amnesias was commended by a number of commentators (Gabriel \& Smith, Markowitsch, Nadel et al., Pickering, Treves). Further support was provided by a review of thalamic infarcts where it was found that anterograde amnesia was best correlated with damage to the mamillothalamic tract (Witter \& Van der Werf). The failure of lesions restricted within the medial dorsal thalamic nucleus to produce anterograde amnesia (Kritchevsky et al. 1987, cited by Kapur) provides more indirect support for the current proposals.

A key test of this proposal is that fornix damage should be sufficient to produce an anterograde amnesia. To investigate this further we have examined a series of 10 people, all of whom have had surgery to remove colloid cysts in the third ventricle (Aggleton, unpublished findings). This condition is of interest as it is sometimes, but not always, associated with loss of the fornix. Clear and quite severe deficits in the learning of new verbal and nonverbal material were found, but this was only in the subgroup of three cases where the fornix was interrupted bilaterally (Fig. R1). Other factors, such as ventricular enlargement, could not

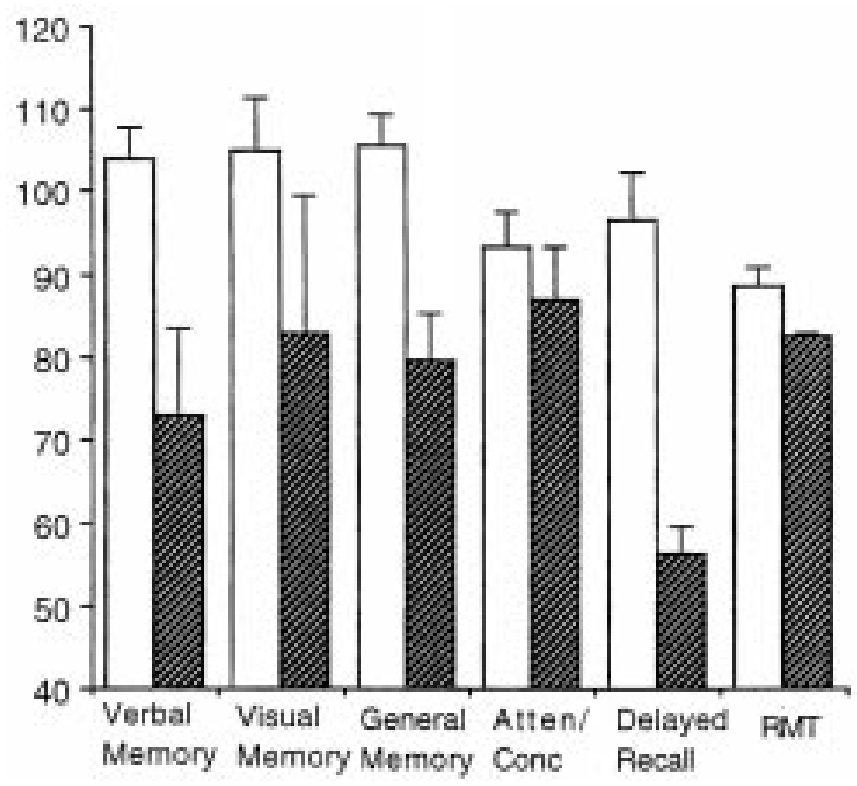

Figure R1. Mean scores (+s.e.) on the subscales of the Wechsler Memory Scale revised (WMSr) and mean total score on the Warrington Recognition Memory test (RMT) for a group of seven subjects (white bars) who required surgical removal of a colloid cyst but in whom the fornix remained intact. The comparison scores (shaded bars) are for three colloid cyst cases where the fornix was interrupted bilaterally. Normal scores on the WMSr are 100, whereas 100 represents the maximum score on the RMT. 
be correlated with memory loss. These results provide further support for other clinical reports linking fornix damage with amnesia (Calabrese et al. 1995; Gaffan \& Gaffan 1991; McMackin et al. 1995).

The significance of the mamillary bodies was questioned by Kapur in light of two new cases where only moderate memory problems were associated with selective mamillary body pathology. Such cases are clearly of enormous interest, but in view of the direct projections from the hippocampus to the anterior thalamic nuclei it is perhaps not surprising that less severe memory problems might be found. The issue of mildness of amnesia was also raised by Zola \& Squire, who questioned how the severity of the amnesia in cases like H. M. could be placed alongside the less severe amnesias in cases such as R. B. with anoxia and associated gross pathology in the CAl subfield of the hippocampus. This concern arises because both suffered from hippocampal dysfunction and this should be the key predictor of their loss of episodic memory. It does not seem appropriate, however, to expect such cases to be comparable in this regard as the extent of hippocampal loss is not equivalent and animal studies have shown that this is potentially a very important factor (Moser et al. 1995; Nunn \& Hodges 1994). There is nothing in our model that precludes this simple conclusion. In addition, the loss of adjacent regions, such as the entorhinal cortex (in H. M.), will further exacerbate the memory loss in such subjects (Corkin et al. 1997).

Perhaps more critical is the suggestion that we have overemphasized the anterior thalamic nuclei at the expense of other thalamic nuclei, in particular the intralaminar nuclei (Mair et al., Witter \& Van der Werf). That damage to the intralaminar nuclei can affect mnemonic performance is now becoming increasingly accepted (Bentivoglio et al. 1997): the work of Mair and his group has been very important in this regard. Nevertheless, the extent to which these regions are important for the encoding and consolidation of new episodic information is far less certain. Many of the animal lesion studies suffer the inevitable problem of either direct damage to adjacent nuclei (including the anterior thalamic nuclei) or damage to tracts passing within the thalamus (including those between the prefrontal cortex and the medial dorsal thalamic nucleus). There are also important questions over the extent to which certain behavioural tests that are sensitive to damage in this region can be regarded as assessing episodic memory (see sect. R4.1).

Although the intralaminar nuclei contribute to memory, and receive direct inputs from the perirhinal cortex (Witter \& Van der Werf), they lack direct hippocampal inputs. This is in marked contrast to the anterior thalamic nuclei. This fact alone points to a qualitatively different contribution to memory. This view is reinforced by Mair et al., who report examples of dissociations between the effects of hippocampal lesions and lesions of the intralaminar region in rats. This can be contrasted with the effects of anterior thalamic lesions, which often resemble hippocampal lesions (see sect. R1). It has been suggested, for example, that the intralaminar and midline nuclei may have much more general regulatory effects on the storage and retrieval of response habits and sensory memories, respectively (Bentivoglio et al. 1997). Such damage may also contribute to the extent of retrograde amnesia (Paller et al. 1997). Thus, although damage to the intralaminar and midline nuclei will exacerbate the memory problems associated with anterior thalamic dysfunction, we regard the intralaminar nuclei as part of an essentially different system and not one that has integral links with the hippocampus.

We proposed that damage to different parts of this hippocampal-diencephalic system will produce similar memory impairments. This is because key components - the hippocampus and the anterior thalamic nuclei - can be regarded as acting in series (Kapur). This is not to imply that the anterior thalamic nuclei do not have their own specific contribution, for example, through ascending cholinergic or reticular innervations, or through connections with the cingulate cortices. Gabriel, who has pioneered much research on the anterior thalamic nuclei and their interaction with the cingulate cortices, argues (Gabriel \& Smith) that our description of the contributions of these nuclei is too narrow (see also Dalrymple-Alford et al.). We readily accept these views, as it was not our goal to provide a comprehensive account of all of the functions of the anterior thalamic nuclei (or the hippocampus for that matter), but to consider the particular issue of how they might contribute to episodic memory in humans. In this regard Dalrymple-Alford et al. make the intriguing suggestion that the contribution of the anterior thalamic nuclei to consolidation might differ from that of the hippocampus in being more transient, and this warrants further investigation.

A different approach is taken by Markowitsch who argues that the proposed system is too narrow as it ignores the contribution of structures such as the amygdala, which appears to be important for aiding the memory of emotionladen material. Although we cannot agree with his assertion that amnesia is to a large extent the failure to remember emotion-laden information, we of course recognise that such mechanisms exist. In a fully comprehensive model one would have to consider how such arousing mechanisms interact with the systems highlighted in our proposal. Perhaps the most pertinent question is whether regions such as the amygdala exert their influence on emotion-laden episodic information via the extended hippocampal-diencephalic system or at some other level (e.g., association cortex). In fact, the amygdala and hippocampus are interconnected, both directly and indirectly (Aggleton 1986), and there is evidence that at least some of the effects of emotional arousal are via this interaction (Cahill \& McGaugh 1996; Packard et al. 1994).

\section{R4. The hippocampal-diencephalic system need not be vital for efficient recognition, as this system is only required for recollection-based recognition ("remembering") so that familiarity-based recognition ("knowing") can still function. The perirhinal cortex, in contrast, is especially important for familiarity-based recognition}

This seemingly simple proposal has many ramifications and so it is not surprising that it was addressed in many of the commentaries. These can be broken down into four main areas: (1) implications for behavioural testing, (2) evidence for this division within recognition in normal and clinical populations, (3) evidence of spared recognition in humans and animals following hippocampal lesions, and (4) evidence that the perirhinal cortex has a specific role in recognition in humans. 
R4.1. Behavioural testing and episodic memory. A number of commentators applauded the division within memory, as it highlighted the weakness of relying on tests of recognition to uncover substrates for episodic memory (Mumby, Nadel et al., Pickering). Others suggested that we had implied that spatial memory and episodic memory were interchangeable (Bures \& Fenton) and therefore questioned the implications of some of our findings concerning selective diencephalic lesions and spatial memory. In fact, we were at pains never to use the terms spatial memory and episodic memory in an interchangeable manner, while acknowledging that spatial information is often crucial to episodic memory. Although tests of spatial memory in rats are of enormous value in examining functional links with the hippocampus, they do not represent direct tests of episodic memory (see Whishaw).

This important point is underlined in a recent, ingenious study by Clayton and Dickinson (1998), which shows that to demonstrate anything closely akin to episodic memory it is necessary to show that the animal has learned not only the where, but also the what and when of an event. This they successfully achieved in scrub jays that were hoarding different kinds of foods that differed in the rates at which they decayed or disappeared once hoarded. To our knowledge, there have been no comparable demonstrations with rats and it would be an enormous advance if such tests could be devised (see also Bures \& Fenton). Mumby offers some useful thoughts on this issue when he discusses the fascinating question of how it might be possible to distinguish two processes within recognition in rats. The study by Clayton and Dickinson (1998) also reveals the limitations of relying on delayed matching or nonmatching tasks to assess episodic memory (Mair et al.), as they do not capture the full extent of episodic processes and can be solved by simpler means.

A closely related issue was raised by Foster when considering the effects of hippocampal lesions on go/no go tasks. He described how hippocampectomised rats were able to perform an unsignalled go/no go task, and argued that because this task has a recall element our proposal should have predicted a deficit. Apart from the potential problems of motor mediation that rats can use to bridge delays, we are reluctant to assume that this task must be episodic in nature, given the stringent demands for such a task (Clayton \& Dickinson 1998). At the same time we are anxious not to fall into the circular trap of implying that because task performance is spared the task does not tax episodic or episodic-like memory (see also Murray).

R4.2. The two-process model of recognition in human neuropsychology. Two of the commentators provide direct support for this model. Hintzman points out how response time measures accord with this model and goes on to suggest that the longer response times for recall-type tasks might be linked to extra processing. The latter proposal is consistent with measurements of neuronal response latencies in recognition tasks in monkeys (Xiang \& Brown 1998). Yonelinas et al. provide further support and go on to address a particular anomaly in the clinical literature. It was pointed out that amnesics with extensive temporal lobe damage may show apparent preservation of familiarity (Verfaellie \& Treadwell 1993), a finding that conflicts with our proposals. By reexamining this issue using signal detection models, evidence has emerged that amnesics with nonfocal temporal lobe damage exhibit deficits in both recollection and familiarity (Yonelinas et al.). Although Pickering has adopted a similar signal detection approach he has failed to find evidence of a division within recognition in normal subjects. It is unclear whether this reflects a weakness in his procedure or a more general weakness in the approach, and there is a need for more studies that adopt this method of analysis.

Other critical comments have come from Knowlton. She argues that "remember" $(\mathrm{R})$ and "know" $(\mathrm{K})$ responses cannot reflect independent processes because of the apparent conversion over time of $\mathrm{R}$ to $\mathrm{K}$ responses. The extent of this conversion indicates that every $\mathrm{R}$ item could also have a $\mathrm{K}$ response and so these are redundant rather than independent processes. In fact, Knowlton and Squire (1995) reported $29 \%$ of items going from $\mathrm{R}$ to $\mathrm{K}$, but only $10 \%$ going from $\mathrm{K}$ to $\mathrm{R}$ during a retention interval of between 10 min and 1 week. The difficulty with this approach lies in the method of distinguishing $\mathrm{R}$ from $\mathrm{K}$, because when using self-report it is almost impossible to give an $\mathrm{R}$ response that does not also assume a $\mathrm{K}$ response, that is, the probability of a "know" response is mathematically constrained by the proportion of "remember" responses (Yonelinas et al.).

A related issue is our claim that familiarity cannot simply be regarded as equivalent to repetition priming. This is potentially important, as priming is often intact in amnesics even though recognition is severely impaired. This relationship was considered by Hintzman and Pickering, who both saw the merits of regarding these processes as distinct (see also Wagner et al. 1998). Indeed, it might be speculated that there are even more component processes to recognition (Nadel et al., Brown \& Xiang 1998). In contrast, Mayes et al. argued that priming does mediate familiarity. This assertion seemed to be based largely on the grounds of parsimony and although we applaud this guiding principle we feel that the present evidence does not support this direct overlap. Foster felt that we were unsure as to whether "familiarity" was a form of implicit memory. This is largely a matter of definition, but given that this information can be used consciously to direct a recognition judgement and to make a "remember"/"know" judgement, we would have to argue that it is not implicit.

R4.3. The effects of hippocampal damage on recognition. The clear prediction from our model is that selective hippocampal damage will spare those recognition tasks that can be performed effectively using familiarity information. In the case of animals the degree of this sparing will depend on the extent to which it is possible for animals to use other forms of information (see R4.1 and Mumby). For rats, the situation appears reasonably consistent, with many studies failing to find delay-dependent deficits, or indeed any deficits, after hippocampectomy (Kesner, Mumby). Although a study by Mumby (cited by Zola \& Squire) did find evidence of an impairment after a retention interval of $120 \mathrm{sec}$, the use of a more difficult condition with a similar delay in the same study failed to reveal any evidence of an impairment (Mumby et al. 1995). Furthermore, in his own commentary Mumby reports a double dissociation in which rats with hippocampal lesions performed normally on a delayed nonmatching-to-sample task in spite of an impairment on a spatial task.

The situation with monkeys is far more complicated because different researchers have used different techniques 
and approaches, factors that are likely to be critical given the sensitivity of the adjacent perirhinal cortex (Gaffan \& Lim 1991). Zola \& Squire present evidence of a mild but significant recognition deficit after selective hippocampectomy (Alvarez et al. 1995) that is comparable to that observed after ischemia (Zola-Morgan et al. 1992). In support of their view they note that the performance of these two sets of animals after a 10 min delay is virtually indistinguishable (means of $68.5 \%$ and $68.1 \%$, respectively). In fact, the mean score for the ischemic group published in Zola-Morgan et al. (1992, Table 1) is 61\% (not 68.1\%). These findings can be directly contrasted with those of a recent study that examined the effects of neurotoxic lesions of the hippocampus on delayed nonmatching to sample (Murray \& Mishkin 1998). Even though there was extensive hippocampal damage there was no evidence of a deficit, even after delays of as long as $40 \mathrm{~min}$. A possible complication with assessing the effects of ischemic damage to the hippocampus is that there may be "cryptic" pathology, but Nadel et al. sensibly warn about relying on this explanation in the absence of other corroborative evidence.

In fact, our model clearly predicts that a recognition deficit should emerge following hippocampectomy if an animal uses episodic recall to aid recognition. This fact might lead to phylogenetic differences because different animals might naturally use different strategies to solve the task. Furthermore, when an animal is confronted by two scenes composed of many common items it may be supposed that familiarity alone becomes less effective and deficits may appear (e.g., Gaffan 1992a; 1992b). Unfortunately, we lack an independent means of establishing when an animal is using episodic recall. Although evidence of episodic memory in birds (Clayton \& Dickinson 1998) must strengthen the likelihood of episodic memory in monkeys, it is premature to assume that tasks such as DNMS (delayed nonmatching-tosample) ever tax this aspect of memory.

Zola \& Squire suggest that pretraining may aid postsurgical performance following hippocampectomy and this may account for the lack of deficit in some studies (e.g., Murray \& Mishkin 1998). Although this may be a factor, it merely indicates that there are nonspecific factors that can disrupt performance and that pretraining helps ameliorate these effects. The key issue is whether hippocampectomy in monkeys can reproduce the consistent recognition deficits observed in amnesia. Amnesics typically show a delay-dependent deficit that is not an artifact of baseline differences (Holdstock et al. 1995). This deficit is apparent after intervals as short as $40 \mathrm{sec}$. There is no evidence that hippocampectomy has comparable effects on delayed nonmatching (or matching) to sample in monkeys.

The proposed model predicts that humans with selective hippocampal damage will show relative sparing of recognition based on familiarity judgements. In a study of people with fornix damage following colloid cysts of the third ventricle (Aggleton, unpublished findings) evidence for this pattern of deficits was found. Although recognition was not normal, it was disproportionately spared (Fig. R1). It is interesting that when subjects were given the Warrington recognition memory test (Warrington 1984) twice so that on the second test (immediately after the first) both stimuli were familiar, a greater deficit was apparent in those subjects with fornix damage. As a result, the overall scores of the groups overlapped on the first test but clearly differed on the second test, leading to a significant interaction.
Mayes et al. also described the recognition performance of a person with selective, but subtotal, hippocampal damage. The predicted sparing of recognition set against impaired recall was observed. Mayes et al. nevertheless argue that the pattern of results is not in strict accord with the model. This is because the person performed poorly on what are described as associative tests of recognition. Clearly, we are in danger of circularity if we simply argue that these exceptions must therefore be episodic in nature, yet the fact that such tasks appear difficult to solve by familiarity alone means that this must be a realistic possibility.

R4.4. The perirhinal cortex and human recognition. On the basis of lesion, electrophysiological, and brain activation data from animals we have assumed that the perirhinal cortex is integral to making familiarity judgements of objects. We have also assumed that the human perirhinal cortex has the same function. This point was considered in detail by Graham \& Hodges who carefully spelled out the current limitations of the clinical evidence, but also provided evidence that is consistent with this view. Mayes et al., however, have described a patient with perirhinal damage who does not fit the proposed model as recognition is relatively intact. This is a finding of great potential importance and we eagerly await a fuller report as to the extent and location of the pathology. Kapur also cites an example of rhinal damage associated with retrograde amnesia rather than recognition loss (Yoneda et al. 1994). As Graham \& Hodges correctly point out, there are uncertainties in identifying the location and extent of perirhinal cortex in the human brain, and until this is resolved the interpretation of such clinical cases will remain difficult.

\section{R5. Although the hippocampus and perirhinal cortex are anatomically linked they are not necessarily dependent on each other for their respective roles in the encoding of episodic information and familiarity-based recognition}

In some respects this might seem the most contentious proposal, as anatomical evidence highlights the extent of the potential interactions between the perirhinal cortex, the parahippocampal cortex, and the hippocampus (Burwell \& Eichenbaum; Knowlton, Witter \& Van der Werf). At the outset we should make it clear that we did not propose that these regions are typically independent of one another, but that there is a continuum of situations where they interact to different degrees. Normally, when information is being learned about complex arrays of individual events the two regions will closely cooperate. At the same time, there may be situations where they can be dissociated and even doubly dissociated. Such double dissociations need not reflect the normal situation, but they highlight the weakness of theories of medial temporal lobe memory systems that assume an automatic interdependency. These views are supported by Graham \& Hodges who cite evidence from studies of the progression of semantic dementia. It was not our intention to "de-emphasize" the cortical inputs to the hippocampus (Burwell \& Eichenbaum), but to show that there are other routes to learning, and that the separate regions can function in an effective, independent manner. This appears to include context-free, long-term semantic 
learning that may occur independent of the hippocampus (Vargha-Khadem et al. 1997).

In the rat brain we have found evidence for such double dissociations between object recognition and spatial memory. Mumby supports this view with reports of similar double dissociations. In contrast, whereas Bilkey argues that perirhinal lesions do lead to consistent spatial memory deficits in rats, Burwell \& Eichenbaum point out a possible explanation for these dissociations as the perirhinal lesions spare the postrhinal cortex, so providing an intact route for spatial information to reach the hippocampus. We have examined these issues at some length and, to our surprise, have consistently found that neurotoxic lesions that essentially eliminate the postrhinal cortex, the perirhinal cortex, and area TE in rats have no effect on T-maze alternation, radial arm maze performance, or Morris water maze performance (Aggleton et al. 1997; Bussey et al. 1999). Not only do they have no effect on standard spatial working memory or reference memory tasks, but we have also found normal radial arm maze performance when a delay of 30 min was interposed after the first 4 choices and the arms of the maze were rotated to nullify intramaze cues. Indeed, the same lesions have resulted in significantly enhanced performance on some spatial tasks (Bussey et al. 1999). These striking results appear inconsistent with the views of Burwell \& Eichenbaum, although it should be noted that we have not included the medial entorhinal cortex in these surgeries.

We remain uncertain as to why Bilkey has consistently observed spatial deficits following perirhinal lesions in rats. It is suggested that rats in our studies may have developed non-mnemonic strategies, but this seems impossible in view of the radial arm maze results with arm rotation and lengthy delays (Bussey et al. 1999). It should also be remembered that the magnitude of the perirhinal deficits observed by Bilkey are very modest when compared to those observed after hippocampectomy. Moreover, the inconsistencies between these results and those of other groups (Aggleton et al. 1997; Ennaceur \& Aggleton 1997) are most likely to reflect procedural differences such as lesion method and extent and type of pretraining. These factors cannot hide the qualitatively different effects of hippocampectomy from joint removal of the perirhinal and postrhinal cortices in rats.

We agree with all of those commentators who noted that the perirhinal (and parahippocampal) cortices have other probable functions (Burwell \& Eichenbaum, Dalrymple-Alford et al., Eacott, Parker, Zola \& Squire). In describing the perirhinal cortex we deliberately chose to emphasize its role in stimulus recognition. A full account of the perirhinal cortex was never intended, and object identification is undoubtedly part of its functions. Instead, our main objective was to consider the hippocampal-diencephalic axis and how this could be linked to both normal episodic memory and disturbances of episodic memory. Eacott does, however, make the important point that the recognition deficit associated with perirhinal damage is markedly reduced when a smaller set size is used and then argues that this region may therefore be specialised for object identification and not familiarity. There is, however, behavioural evidence that animals solve the DNMS task with small set size in ways that differ from those used with trial unique stimuli (Mishkin \& Delacour 1975). Furthermore, with frequently repeated stimuli the task is most easily solved using rapidly decaying recency information, and such information is available in neurons in area TE of the temporal cortex (Baylis et al. 1987; Fahy et al. 1993a; 1993b). The finding of an impairment at even the shortest delays when using a large set size (Eacott et al. 1994) again indicates a perceptual component to the deficit that is consistent with a perirhinal involvement in stimulus identification, as well as stimulus familiarity (Brown \& Xiang 1998). A further significance of the findings concerning set size is that they serve to distinguish the possible contributions of this cortical region from those of the fornix, where familiarity with the stimuli may exacerbate rather than spare the recognition deficit (Owen \& Butler 1984). This contrasting pattern of results can be accommodated in the present proposal if it is assumed that fornix damage spares familiarity, and that stimulus repetition in primates lessens the value of this cue. In contrast, perirhinal lesioned animals cannot use familiarity and so stimulus repetition will not heighten task difficulty, as it would in normal animals.

\section{R6. There are two parallel temporal-thalamic pathways, one from the hippocampus to the anterior thalamic nuclei, the other from perirhinal cortex to the medial dorsal thalamic nucleus. The second of these pathways underlines familiarity-based recognition}

Evidence concerning the first of these pathways has already been discussed (see sect. R3) but the nature of the involvement of the medial dorsal thalamic nucleus (MD) in recognition remains more a matter of debate. Clinical studies have proved to be of little help, as circumscribed damage to this region is extraordinarily rare (Markowitsch). Experimental studies have repeatedly shown, however, that damage to the medial dorsal nucleus can impair recognition in monkeys (Aggleton \& Mishkin 1983b; Parker et al. 1997; Zola-Morgan \& Squire 1985a), but the underlying reasons for this deficit are more difficult to determine. The key question is whether the involvement of MD depends on inputs from the perirhinal (and entorhinal) cortices or whether the recognition deficit is qualitatively different (Eacott, Parker, Mair et al.), in particular, whether the MD deficit may be more closely linked with frontal lobe dysfunction rather than perirhinal cortex dysfunction.

Various commentators noted that the current evidence for a crucial perirhinal-medial dorsal circuit is not compelling. Although reciprocal pathways do exist in primates (Aggleton et al. 1986a; Markowitsch et al. 1985; Russchen et al. 1987), these are only light (Witter \& Van der Werf). Furthermore, the severity of the recognition deficit after medial dorsal damage is markedly less than that observed after medial temporal damage (Parker), a fact first noted by Aggleton and Mishkin (1983b). For this reason we postulated that the projections from the perirhinal cortex to the prefrontal cortex were also involved in familiarity recognition (see Fig. 2, target article), even though they need not be essential (Gaffan \& Eacott 1995). Other relevant evidence has come from recent comparisons between the recognition deficits seen after medial thalamic lesions and perirhinal lesions (Parker et al. 1997). It was found that MD lesions, like perirhinal lesions, lead to a deficit for large but not small set sizes. This is clearly consistent with the postu- 
lated link between the two regions. It should also be noted that it is hard to argue that this thalamic set size effect is the result of a perceptual deficit, and so shows a weakness with the assumption that the same effect following perirhinal lesions must be perceptual (Eacott).

Another approach is to disconnect the medial dorsal thalamic nucleus by combining a unilateral lesion in MD with a unilateral lesion in the perirhinal cortex in the contralateral hemisphere. A recent study using this approach showed that this surgery impaired DMS (Parker \& Gaffan 1998a), again implicating the perirhinal-MD link. An alternative means of disconnecting the perirhinal cortex from MD is to cut the efferent pathway from the perirhinal and entorhinal cortices as it passes through the external capsule in the rostral temporal stem (Goulet et al. 1998). A consideration of relevant experiments reveals an uncertain picture. In both studies (Bachevalier et al. 1985a; Parker \& Gaffan 1998a) a dorsal amygdala cut, that is presumed to have disrupted inputs from perirhinal cortex to MD, had only mild effects on tests of recognition. This has been interpreted as indicating that the key connections are those with the prefrontal cortex (Parker), even though the direct connections may not be critical (Gaffan \& Eacott 1995). One problem is that null results with disconnection studies are typically very difficult to interpret unless it can be demonstrated that the key connections have been fully severed. In the case of crossed lesion experiments (Parker \& Gaffan 1998a) there is an additional assumption that the functional pathways are essentially ipsilateral, but it is likely that there are light crossed projections and these might support performance.

The important point was made that it might be more accurate to regard the contribution of MD as more a consequence of its connections to the prefrontal cortex than to the temporal lobe (Parker). This is a view that we have growing sympathy for, and it has been reinforced by studies that highlight the similarity between the effects of MD lesions and prefrontal lesions in animals (Bachevalier \& Mishkin 1986; Hunt \& Aggleton 1998). The difficulty with this view comes from the clinical literature, which shows that the recognition deficit associated with diencephalic amnesias is typically as severe as that observed in temporal lobe amnesias (Aggleton \& Shaw 1996), whereas the effects of prefrontal cortex damage on recognition are typically mild. One suggestion is that the importance of the prefrontal cortex has been masked by clinical cases that emphasize the effects of partial cortical damage and that the consequences of extensive, bilateral prefrontal cortical loss remain poorly understood (Parker). We are aware of these problems, and it is the case that interactions between the perirhinal cortex and prefrontal cortex and between MD and prefrontal cortex figure prominently in our putative system underling familiarity recognition (Fig. 2, target article). There is clearly growing evidence that these interactions should be emphasised even more, and this may prove to be partly at the expense of the connections between perirhinal cortex and MD.

Finally, Witter \& Van der Werf make the case that it is the intralaminar and midline nuclei, rather than MD, that are linked with the perirhinal cortex in signalling familiarity. They present preliminary data from a patient who shows deficits on face recognition but is able to preserve visual association learning following a thalamic infarct. We await more details on this case, in particular, information con- cerning the location of the infarct and its possible effect on fibres of passage. It will also be important to ensure that the preserved learning was not a result of verbal mediation.

\section{R7. The traditional distinction between temporal lobe and diencephalic amnesias is misleading as both have damage to the same functional system}

This change of emphasis was welcomed by a number of commentators (Kapur, Pickering) and it is seen as an inevitable consequence of our better understanding of the contributions of the fornix to memory. The issue of how amnesias may be of different severity was noted in some commentaries (Dalrymple-Alford et al., Kapur, Zola \& Squire), and the growing evidence concerning the possible contributions of the prefrontal cortex (Parker, Knowlton) and the intralaminar thalamic nuclei (Mair et al.) helps to emphasize how difficult it may be to interpret the effects of pathologies in multiple sites. The potential impact of even subtotal pathology in the anterior thalamic nuclei was noted (Dalrymple-Alford et al.) and this may serve as a useful prompt to reexamine the status of this nuclear group in a variety of conditions that disrupt memory (e.g., various dementias and Korsakoff's syndrome). Recent imaging studies have helped to show how pathologies in one region can lead to extensive abnormalities in other, distal sites (Markowitsch et al. 1997; Paller et al. 1997) and this is likely to prove a valuable means of addressing this highly complex issue. Finally, Pickering and Kapur both commented on the need for an agreed definition of amnesia, and whether impaired recognition is a necessary feature of this syndrome. Clearly, we do not think this is a necessary feature, but we agree that there needs to be greater awareness of the problems of definition.

\section{R8. The hippocampal-diencephalic system is required for the encoding of episodic information permitting it to be set in its spatial and temporal context}

A number of commentators have offered alternative accounts of the contributions of the hippocampus to mnemonic functions (Burwell \& Eichenbaum, Gabriel \& Smith, Mayes et al., Murray, Nadel et al., Parker, Whishaw). We should, at the outset, reiterate the fact that the target article was focussed on the hippocampal-rostral thalamic pathways and was not intended as a comprehensive review of hippocampal function. Two of these accounts emphasized the importance of this region for remembering movements in space (Parker, Whishaw), and this view can readily be used to help explain the head direction information that passes from the anterior thalamic group to the hippocampus (Taube 1995). It also accords with other studies indicating a role in the memory for instrumental responses (Gaffan et al. 1984; Rupniak \& Gaffan 1987). A number of studies into the effects of fornix lesions in animals do reveal limitations with this as a complete account, however. Thus although deficits are found for conditional and discrimination tasks where the key manipulations are the spatial disposition of the stimuli (Gaffan 1991; Gaffan \& Harrison 1989), the animal's movements provide little information. Furthermore, the recent report that hippocampectomy 
does not disrupt the ability of monkeys to perform accurate positional responses that differ from trial to trial (Murray \& Mishkin 1998) appears incompatible with the suggestion (Parker) that deficits on the object-in-place discrimination reflect a failure of the hippocampal-thalamic axis to provide ideothetic (self-motion) information that can help distinguish the different stimuli. If on the other hand Parker is suggesting that the hippocampus is required for remembering both the movements and the particular situations, then the links between scene memory, ideothetic information, and episodic memory become considerably stronger.

Other commentators have argued that a role in episodic memory is too limiting a description of hippocampal-anterior thalamic function (Burwell \& Eichenbaum; Gabriel \& Smith), and does not account for the full array of deficits observed after hippocampal damage in rats. It has to be remembered that we still lack convincing evidence of episodic memory in rats, and so for this species the description must be in terms of functions that can ultimately form a basis for episodic memory. Thus the creation of "scenes" both accounts for the deficits at the level of this species and permits phylogenetic development into something we call episodic memory. In a similar vein, Murray argued that the hippocampus is important for associations with memory representations of stimuli not present in the "here and now."

Whishaw argues that the principal function of the hippocampus is to permit accurate navigation in space by means of path integration. Critically, it is argued that hippocampectomized rats still have place memory, and so the link from place memory in rats to scene memory in primates is seriously weakened. One of the problems with this proposal is that rats have a number of different ways of solving a spatial task such as finding a hidden platform in a water maze. The most obvious method is to identify the position with respect to a configuration of landmarks (a "cognitive map" approach), but another is to learn its position with respect to a single landmark. There is compelling evidence that both strategies exist, and there is recent evidence that this second strategy is independent of the hippocampus (Pearce et al. 1998). If the training methods adopted by Whishaw encourage this landmark strategy, then the pattern of performance he describes does not conflict with our initial proposals.

\section{R9. The prefrontal cortex interacts with both the hippocampal-anterior thalamic and the perirhinal-medial dorsal thalamic systems at a variety of levels, thereby engaging efficient strategies for recall}

A number of commentators wished that interactions with prefrontal cortex had been more detailed (Foster, Knowlton, Parker). To provide a comprehensive review of the contributions of the prefrontal cortex to learning would have been beyond the goals of the target article. Nevertheless, there is recent evidence (see sect. R6) to suppose that the prefrontal cortex has an important role in recognition as well as recall. There is also considerable evidence from functional imaging studies implicating different regions of the prefrontal cortex in various aspects of memory. Indeed, it seems highly likely that the contributions of this region have been systematically underestimated as extensive, but circumscribed pathologies are rarely if ever described in humans. Furthermore, animal studies, and especially those using rats, will inevitably fail to reveal the full potential importance of the prefrontal cortex given its phylogenetic development. Although summary Figures 1 and 2 (target article) both included prefrontal cortex their interactions remain to be examined and understood in much greater detail. This represents one of several areas for research that we hope might be stimulated by our target article.

\section{R10. Concluding remarks}

A very deliberate aspect of our review was the use of data from a variety of methodologies and species. There are, of course, well-recognised problems in moving across species, and there may have been occasions when we failed to point out the implications of this practice (Kapur). We feel that it is important, however, to show how these data interlink, as certain methodologies are much more appropriate for some species than others. An example of this is the use of immediate early gene activation studies in rats. Bures \& Fenton commented on this approach and correctly noted that to complete the link between increased c-fos activation and recognition it is important to have simultaneous behavioural indices of novelty detection. We are currently addressing this issue. Bures \& Fenton also argued that the paired viewing procedure might be flawed because the combined view from the two eyes might produce a novel configuration, so making all conditions "novel." This is an ingenious notion, but the fact that we find very similar results when the same studies are run in a between-subjects design (Zhu et al. 1995b) argues against this view. Furthermore, the c-fos results are highly consistent with the findings from single unit recording studies in both rats and monkeys (Zhu et al. 1995a).

Several commentators (Kesner, Knowlton, Mumby) suggested that we had misinterpreted the apparent differences between the perirhinal cortex and the hippocampus because the dissociations between these regions reflect differences in the nature of the afferent information and not the form of processing that occurs within these structures. Thus it is because the hippocampus receives a considerable amount of spatial information, in contrast to the perirhinal cortex, that these two structures differ functionally. This is an appealing notion, but it suffers a number of serious drawbacks. To take the specific example provided above, it does not account for the failure of combined perirhinal and postrhinal lesions to affect a variety of spatial tasks in rats (Aggleton et al. 1997; Bussey et al. 1999), even though the combined cortical regions undoubtedly receive much spatial information. At a more general level, this proposal flies in the face of the established notion that different cytoarchitectures reflect different modes of processing. A consideration of the intrinsic connections of the ventral temporal cortex reveals a system of information convergence (e.g., in columns) that can be contrasted with the divergence of information that characterises the hippocampus (Brown 1990). Thus it seems more appropriate to regard these structures as having qualitatively different processing capabilities.

Finally, Nadel et al. make a number of imaginative speculations concerning how our proposals might be applied to recall processes and retrograde amnesia. These include the 
notion that there might be two routes by which the hippocampus can reactivate cortical representations, and the consequences of dysfunctions in just one of these routes. We deliberately did not consider retrograde amnesia in our review, principally because there is sufficient evidence that anterograde amnesia can (occasionally) occur in the absence of retrograde amnesia. Nevertheless, any attempt to explain the nature of new learning will have implications both for the fate of old memories and for the subsequent reactivation of new memories. This is just one example of how our initial proposals may lead to implications that we had not anticipated, and we look forward to other aspects of our model being explored and tested.

\section{References}

Letters " $a$ " and " $r$ " appearing before authors' initials refer to target article and response, respectively.

Aggleton, J. P. (1986) A description of the amygdala-hippocampal interconnections in the macaque monkey. Experimental Brain Research 64:515-26. [rJPA]

Aggleton, J. P., Desimone, R. \& Mishkin, M. (1986a) The origin, course, and termination of the hippocampo-thalamic projections in the macaque. Journal of Comparative Neurology 243:409-21. [arJPA, MPW]

Aggleton, J. P., Hunt, P. R., Nagle, S. \& Neave, N. (1996) The effects of selective lesions within the anterior thalamic nuclei on spatial memory in the rat. Behavioural Brain Research 81:189-98. [aJPA, JCD-A]

Aggleton, J. P., Hunt, P. R. \& Rawlins, J. N. P. (1986b) The effects of hippocampal lesions upon spatial and non-spatial tests of working memory. Behavioural Brain Research 19:133-46. [aJPA]

Aggleton, J. P., Hunt, P. R. \& Shaw, C. (1990) The effects of mammillary body and combined amygdalar-fornix lesions on tests of delayed non-matching- tosample in the rat. Behavioural Brain Research 40:145-57. [aJPA]

Aggleton, J. P., Keen, S., Warburton, E. C. \& Bussey, T. J. (1997) Extensive cytotoxic lesions of the rhinal cortices impair recognition but spare spatial alternation in the rat. Brain Research Bulletin 43:279-87. [arJPA, DKB]

Aggleton, J. P., Keith, A. B., Rawlins, J. N. P., Hunt, P. R. \& Sahgal, A. (1992) Removal of the hippocampus and transection of the fornix produce comparable deficits on delayed non-matching to position by rats. Behavioural Brain Research 52:61-71. [aJPA]

Aggleton, J. P. \& Mishkin, M. (1983a) Visual recognition impairment following medial thalamic lesions in monkeys. Neuropsychologia 21:189-97. [aJPA, AP]

(1983b) Memory impairments following restricted medial thalamic lesions in monkeys. Experimental Brain Research 52:199-209. [arJPA, AP]

(1985) Mamillary-body lesions and visual recognition in monkeys. Experimental Brain Research 58:190-97. [aJPA]

Aggleton, J. P., Neave, N., Nagle, S. \& Hunt, P. R. (1995a) A comparison of the effects of anterior thalamic, mamillary body and fornix lesions on reinforced spatial alternation. Behavioural Brain Research 68:91-101. [aJPA]

Aggleton, J. P., Neave, N., Nagle, S. \& Sahgal, A. (1995b) A comparison of the effects of medial prefrontal, cingulate cortex, and cingulum bundle lesions on tests of spatial memory: Evidence of a double dissociation between frontal and cingulum bundle contributions. Jounal of Neuroscience 15:7270-81. [aJPA]

Aggleton, J. P., Nicol, R. M., Huston, A. E. \& Fairbairn, A. F. (1988) The performance of amnesic subjects on tests of experimental amnesia in animals: Delayed matching-to-sample and concurrent learning. Neuropsychologia 26:265-72. [aJPA]

Aggleton, J. P. \& Sahgal, A. (1993) The contribution of the anterior thalamic nuclei to anterograde amnesia. Neuropsychologia 31:1001-19. [aJPA, JCD-A]

Aggleton, J. P. \& Saunders, R.C. (1997) The relationships between temporal lobe and diencephalic structures implicated in anterograde amnesia. Memory 5:49-71. [arJPA]

Aggleton, J. P. \& Shaw, C. (1996) Amnesia and recognition memory: A reanalysis of psychometric data. Neuropsychologia 34:51-62. [arJPA, ADP]

Alvarez, P., Zola-Morgan, S. \& Squire, L. R. (1995) Damage limited to the hippocampal region produces long-lasting memory impairment in monkeys. Journal of Neuroscience 15:3796-807. [arJPA, DJM, SMZ]

Amarel, D. G. (1987) Memory: Anatomical organization of candidate brain regions. In: Handbook of physiology: The nervous system, ed. V. B. Mountcastle, F. Plum \& S. R. Geiger. American Psychological Society. [EEK]

Armstrong, E. (1986) Enlarged limbic structures in the human brain: The anterior thalamus and medial mamillary body. Brain Research 362:394-97. [JCD-A]
Armstrong, E., Clarke, M. R. \& Hill, E. M. (1987) Relative size of the anterior thalamic nuclei differentiates anthropoids by social system. Brain Behavior and Evolution 30:263-71. [JCD-A]

Assal, G., Probst, A., Zander, E. \& Rabionwicz, T. (1976) Syndrome amnesique par infiltration tumorale. Archiv Suisse Neurology Neurochirurgica Psychiatry 119:317-24. [aJPA]

Astur, R. S., Mumby, D. G. \& Sutherland, R. J. (1995) Perirhinal cortex damage: Effects of acquisition and retention of object and place discriminations in rats. Society for Neuroscience Abstracts 21:1935. [MJE]

Atkinson, R. C. \& Juola, J. F. (1974) Search and decision processes in recognition memory. In: Contemporary developments in mathematical psychology: I. Learning, memory and thinking, ed. R. C. Atkinson, R. D. Luce, D. H. Krantz $\&$ P. Suppes. Freeman. [APY]

Bachevalier, J. \& Meunier, M. (1996) Cerebral ischemia: Are the memory deficits associated with hippocampal cell loss? Hippocampus 6:553-60. [aJPA, SMZ]

Bachevalier, J., \& Mishkin, M. (1986) Visual recognition impairment follows ventromedial but not dorsolateral prefrontal lesions in monkeys. Behavioural Brain Research 20:249-61. [arJPA]

(1989) Mnemonic and neuropathological effects of occluding the posterior cerebral artery in Macaca mulatta. Neuropsychologia 27:83-105. [aJPA]

Bachevalier, J., Parkinson, J. K. \& Mishkin, M. (1985a) Visual recognition in monkeys. Effects of separate vs. combined transection of fornix and amygdalofugal pathways. Experimental Brain Research 57:554-61. [arJPA, AP].

Bachevalier, J., Saunders, R. C. \& Mishkin, M. (1985b) Visual recognition in monkeys. Effects of transection of fornix. Experimental Brain Research 57:547-53. [aJPA]

Barlow, J. S. (1964) Inertial navigation as a basis of animal navigation. Journal of Theoretical Biology 6:76-117. [IQW]

Barnes, C. A. (1988) Spatial learning and memory processes: The search for their neurobiological mechanisms in the rat. Trends in Neuroscience 11:163-69. [aJPA]

Baxendale, S. A. (1997) The role of the hippocampus in recognition memory. Neuropsychologia 35:591-98. [aJPA]

Baylis, G. C., Rolls, E. T. \& Leonard, C. M. (1987) Functional subdivisions of the temporal lobe neocortex. The Journal of Neuroscience 7:330-42. [rJPA]

Beason-Held, L. L., Rosene, D. L., Killiany, R. J. \& Moss, M. B. (in press) Ibotenic acid lesions of the hippocampal formation produce memory deficits in the rhesus monkey. Hippocampus. [SMZ]

Beason-Held, L., Rosene, D. L. \& Moss, M. (1993) Memory deficits associated with ibotenic acid lesions of the hippocampal formation in rhesus monkeys. Society for Neuroscience Abstracts 19:438. [aJPA]

Benedek, L. \& Juma, A. (1941) Korsakowsyndrom bei den Geschwulsten des Zwischenhirns. Archive Psychiatry 114:366-76. [aJPA]

Bentivoglio, M., Aggleton, J. P. \& Mishkin, M. (1997) The thalamus and memory formation. In: Thalamus, ed. M. Steriade, E. G. James \& D. A. McCormick. Elsevier. [rJPA]

Beracochea, D. \& Jaffard, R. (1991) Effects of chronic ethanol consumption associated or not with experimental anterior thalamic lesions on spontaneous sequential alternation in mice. Neuroscience Letters 134:45-48. [JCD-A]

(1995) Effects of anterior thalamic lesions on spatial memory in mice. Neuroreport 5:917-20. [aJPA, JCD-A]

Beracochea, D. J., Jaffard, R. \& Jarrard, L.E. (1989) Effects of anterior or dorsomedial thalamic ibotenic lesions on learning and memory in rats. Behavioral and Neural Biology 51:364-76. [aJPA, JCD-A]

Bloomfield, S. A. \& Sherman, S. M. (1989) Dendritic current flow in relay cells and interneurons of the cat's lateral geniculate nucleus. Proceedings of the National Academy of Sciences USA 86(10):3911-14. [EEK]

Böttger, S., Prosiegel, M., Steiger, H. J. \& Yassouridis, A. (1998) Neurobehavioural disturbances, rehabilitation outcome, and lesion site in patients after rupture and repair of anterior communicating artery ancurysm. Journal of Neurology, Neurosurgery and Psychiatry 65:93-102. [HJM]

Brion, S. \& Mikol, J. (1978) Atteinte du noyou lateral dorsal du thalamus et syndrome de Korsakoff alcoolique. Journal of the Neurological Sciences 38:249-61. [aJPA]

Brito, G. N. O., Thomas, G. J., Davis, B. J. \& Gingold, S. I. (1982) Prelimbic cortex, mediodorsal thalamus, septum, and delayed alternation in rats. Experimental Brain Research 46:52-58. [aJPA]

Brown, M. W. (1982) Effect of context on the responses of single units recorded from the hippocampal region of behaviourally trained monkeys. In: Neuronal plasticity and memory formation, IBRO Monograph Series Vol. 9, ed. C. Ajmone-Marsan \& H. Matthies. Raven Press. [aJPA]

(1990) Why does the cortex have a hippocampus? In: Learning and computational neuroscience: Foundations of adaptive networks, ed. M. Gabriel \& J. Moore. MIT Press. [arJPA]

(1996) Neuronal responses and recognition memory. Seminars in the Neurosciences 8:23-32. [aJPA] 
References/Aggleton \& Brown: Episodic memory, amnesia, and hippocampus

Brown, M. W., Wilson, F. A. W. \& Riches, P. (1987) Neuronal evidence that inferomedial temporal cortex is more important than hippocampus in certain processes underlying recognition memory. Brain Research 409:158-62. [aJPA, MJE]

Brown, M. W. \& Xiang, J.-Z. (1998) Recognition memory: Neuronal substrates of the judgement prior occurrence. Progress in Neurobiology 55:149-89. [arJPA]

Bruin, J. P .C. de, Sanchez-Santed, F., Heinsbroek, R. P. W., Donker, A. \& Postmes, P. (1994) A behavioural analysis of rats with damage to the medial prefrontal cortex using the Morris water maze: Evidence for behavioural flexibility, but not for impaired spatial navigation. Brain Research 652:322-33. [aJPA]

Buckley, M. J. \& Gaffan, D. (1997) Impairment of visual object discrimination learning after perirhinal cortex ablation. Behavioral Neuroscience 111:467-75. [MJE]

(1998a) Perirhinal cortex ablation impairs visual object identification. Journal of Neuroscience 18:2268-75. [MJE]

(1998b) Perirhinal cortex ablation impairs configural learning and pairedassociate learning equally. Neuropsychologia 36:535-46. [MJE]

(1998c) Learning and transfer of object-reward associations and the role of the perirhinal cortex. Behavioral Neuroscience 112:15-23. [MJE]

Bunsey, M. \& Eichenbaum, H. (1993) Critical role of the parahippocampal region for paired associates learning in rats. Behavioral Neuroscience 107:740-47. [MJE]

(1996) Conservation of hippocampal memory function in rats and humans. Nature 379:255-57. [RDB, SMZ]

Burk, J. A. \& Mair, R. G. (1998a) Thalamic amnesia reconsidered: Excitotoxic lesions of the intralaminar nuclei, but not the mediodorsal nucleus disrupt place DMTS performance in the rat (Rattus norvegicus). Behavioral Neuroscience 112:54-67. [RGM]

(1998b) Delayed matching-to-sample trained with retractable levers is impaired by lesions of the intralaminar or ventromedial but not the laterodorsal thalamic nuclei (submitted). [RGM]

Burwell, R. D. \& Amaral, D. G. (1998a) Cortical afferents of the perirhinal, postrhinal, and entorhinal cortices. Journal of Comparative Neurology 398(2):179-205. [RDB]

(1998b)The perirhinal and postrhinal cortices of the rat: Interconnectivity and connections with the entorhinal cortex. Journal of Comparative Neurology 391(3):293-321. [RDB]

Burwell, R. D., Caballero, M., Witter, M. P. \& Amaral, D. G. (1994) A PHA-L study of the perirhinal projection to the thalamus in the rat. Society for Neuroscience Abstracts 20:1415. [MPW]

Burwell, R. D., Witter, M. P. \& Amaral, D. G. (1995) Perirhinal and postrhinal cortices of the rat: A review of the neuroanatomical literature and comparison with findings from the monkey brain. Hippocampus 5:390-408. [aJPA]

Bussey, T. J., Muir, J. \& Aggleton, J. (1999) Functionally dissociating aspects of event memory: The effects of combined perirhinal and postrhinal cortex lesions on object and place memory in the rat. The Journal of Neuroscience 19:495-503. [rJPA]

Butters, N., Salmon, D. P., Munro Cullum, C., Cairns, P., Tröster, A. I., Jacobs, D. Moss, M. \& Cermak, L. S. (1988) Differentiation of amnesic and demented patients with the Wechsler Memory Scale-Revised. Clinical Neuropsychology 2:133-48. [aJPA]

Byatt, G. \& Dalrymple-Alford, J. C. (1996) Both anteromedial and anteroventral thalamic lesions impair radial-maze learning in rats. Behavioral Neuroscience 110:1335-48. [aJPA, JCD-A]

Cahill, L., Babinsky, R., Markowitsch, H. J. \& McGaugh, J. L. (1995) Involvement of the amygdaloid complex in emotional memory. Nature 377:295-96. [HJM]

Cahill, L. \& McGaugh, J. L. (1996) Modulation of memory storage. Current Opinion in Neurobiology 6:237-42. [rJPA]

Cai, H. B. \& Lo, F. S. (1996) Passive cable properties of relay cells in dorsal lateral geniculate nucleus of the rabbit. Journal Sheng Li Hsueh Pao 48(6):577-80 [EEK]

Cairns, H. \& Mosberg, W. H. (1951) Colloid cyst of the third ventricle. Surgery Gynecology, Obstetrics 92:546-70. [aJPA]

Calabrese, P., Haupts, M., Markowitsch, H. J. \& Gehlen, W. (1993) The cognitiveamnesic performance profile of a patient with bilateral asymmetrical thalamic infarction. International Journal of Neuroscience 71:101-106. [aJPA]

Calabrese, P., Markowitsch, H .J., Harders, A. G., Scholz, M. \& Gehlen, W. (1995) Fornix damage and memory. A case report. Cortex 31:555-64. [arJPA]

Cameron, A. S. \& Archibald, Y. M. (1981) Verbal memory deficit after left fornix removal: A case report. International Journal of Neuroscience 12:201. [aJPA]

Carmel, P. W. (1985) Tumours of the third ventricle. Acta Neurochirurgica 75:136-46. [aJPA]

Cassaday, H. J. \& Rawlins, J. N. P. (1995) Fornix-fimbria section and working memory deficits in rats: Stimulus complexity and stimulus size. Behavioral Neuroscience 109:594-606. [aJPA]
(1997) The hippocampus, objects and their contexts. Behavioral Neuroscience 111:1228-44. [aJPA, JKE]

Castaigne, P., Lhermitte, F., Buge, A., Escourolle, R., Hauw, J. J. \& Lyon-Caen, O. (1981) Paramedian thalamic and midbrain infarcts: Clinical and neuropathological study. Annals of Neurology 10:127-48. [aJPA]

Cave, C. B. \& Squire, L. R. (1992) Intact verbal and nonverbal short-term memory following damage to the human hippocampus. Hippocampus 2:151-64. [aJPA]

Cermak, L. S., Talbot, N., Chandler, K. \& Reale Wolbarst, L. (1985) The perceptual priming phenomenon in amnesia. Neuropsychologia 23:615-22. [aJPA]

Chen, H.-C. \& Burwell, R. D. (1996) An anterograde tract-tracing study of the perirhinal and postrhinal cortical projections to the thalamus in the rat brain. Journal of Undergraduate Research 3:47-68. [MPW]

Clark, R. E., Teng, E., Squire, L. R. \& Zola, S. (1996) The visual pairedcomparison task and the medial temporal lobe memory system. Society for Neuroscience Abstract 22:116.10. [aJPA, SMZ]

(1997) Perirhinal damage impairs memory on the visual paired-comparison task. Society for Neuroscience Abstract 23:12.8. [aJPA]

Clarke, S., Assal, G., Bogousslavsky, J., Regli, F., Townsend, D. W., Leenders, K. L. \& Blecic, S. (1994) Journal of Neurology, Neurosurgery and Psychiatry $57: 27-34$. [aJPA]

Clayton, N. S. \& Dickinson, A. (1998) Episodic-like memory during cache recovery by scrub jays. Nature 395:272-74. [rJPA]

Cohen, N. J. \& Eichenbaum, H. (1993) Memory, amnesia and the hippocampal system. MIT Press. [DJM]

Colmant, H. J. (1965) Enzephalopien bei chronischem Alkoholismus, insbesondere Thalamusbefunde bei Wernickescher Enzephalopathie. F. Enke. [aJPA]

Colombo, M. \& Gross, C. G. (1996) Hippocampus, delay neurons, and sensory heterogeneity. Behavioral and Brain Sciences 19:766-67. [JCD-A]

Corkin, S. (1984) Lasting consequences of bilateral medial temporal lobectomy: Clinical course and experimental findings in HM. Seminars in Neurology $4: 249-59 . \quad[\mathrm{NK}]$

Corkin, S., Amaral, D. G., González, R. G., Johnson, K. A. \& Hyman, B. T. (1997) H. M.'s medial temporal lobe lesion: Findings from magnetic resonance imaging. Journal of Neuroscience 17:3964-79. [rJPA, KSG]

Cramon, D. Y., von Hebel, N. \& Schuri, U. (1985) A contribution to the anatomical basis of thalamic amnesia. Brain 108:993-1008. [aJPA]

Crick, F. \& Jones, E. (1993) The backwardness of human neuroanatomy. Nature 361:109-10. [NK]

Cruce, J. A. F. (1975) An autoradiographic study of the projections of the mammillothalamic tract in the rat. Brain Research 85:211-19. [aJPA]

Cummings, J. L., Tomiyasu, S., Read, S. \& Benson, D. F. (1984) Amnesia with hippocampal lesions after cardiopulmonary arrest. Neurology 34:679-81. [aJPA]

Curran, T. \& Hintzman, D. L. (1995) Violations of the independence assumption in process dissociation. Journal of Experimental Psychology: Learning, Memory, and Cognition 21:531-47. [ADP]

Daum, I. \& Ackermann, H. (1994) Frontal-type memory impairment associated with thalamic damage. International Journal of Neuroscience 77:187-98. [aJPA]

Day, L. B. \& Schallert, T. (1996) Anticholinergic effects on acquisition of place learning in the Morris water task: Spatial mapping deficit or inability in inhibit nonplace strategies? Behavioral Neuroscience 110:998-1005. [IQW]

Dehn, D. M. \& Engelkamp, J. (1997) Process dissociation procedure: Double dissociations following divided attention and speeded responding. Quarterly Journal of Experimental Psychology 50A:318-36. [aJPA]

DeJong, R. N., Itabashi, H. H. \& Olson, J. R. (1969) Memory loss due to hippocampal lesion. Archives of Neurology 20:339-48. [aJPA]

Delay, J. \& Brion, S. (1969) Le syndrome de Korsakoff. Masson. [aJPA, JKF, AP]

Delbecq-Derousne, J., Beauvois, M. \& Shallice, T. (1990) Preserved recall versus impaired recognition. Brain 113:1054-74. [NK]

DeLuca, J. \& Diamond, B. J. (1995) Aneurysm of the anterior communicating artery: A review of neuroanatomical and neuropsychological sequelae. Journal of Clinical and Experimental Neuropsychology 17:100-121. [HJM]

Diamond, B. J., DeLuca, J. \& Kelly, S. M. (1997) Memory and executive functions in amnesic and non-amnesic patients with aneurysms of the anterior communicating artery. Brain 120:1015-25. [HJM]

Diesfeldt, H. F. A. (1992) Impaired and preserved semantic memory functions in dementia. In: Memory functioning in dementia, ed. L. Backman. Elsevier. [KSG]

Dolan, R. J. \& Fletcher, P. C. (1997) Dissociating prefrontal and hippocampa function in episodic memory encoding. Nature 388:582-85. [aJPA]

Donaldson, W. (1996) The role of decision processes in remembering and knowing. Memory and Cognition 24:523-33. [aJPA]

Dum, R. P. \& Strick, P. L. (1993) Cingulate motor areas. In: Neurobiology of cingulate cortex and limbic thalamus, ed. B. A. Vogt \& M. Gabriel. Birkhauser. [EEK] 
Dusek, J. A. \& Eichenbaum, H. (1997) The hippocampus and memory for orderly stimulus relations. Proceedings of the National Academy of Sciences USA 94:7109-14. [RDB]

Dusoir, H., Kapur, N., Byrnes, D. P., McKinstry, S. \& Hoare, R. D. (1990) The role of diencephalic pathology in human memory disorder. Brain 113:1695-706. [aJPA]

Duva, C. A., Floresco, S. B., Wunderlich, G. P., Lao, T. L., Pinel, J. P. J. \& Phillips, A. G. (1997) Disruption of spatial but not object-recognition memory by neurotoxic lesions of the dorsal hippocampus in rats. Behavioral Neuroscience 111:1184-96. [aJPA]

Düzel, E., Yonelinas, A. P., Mangun, G. R., Heinze, H. \& Tulving, E. (1997) Eventrelated brain potential correlates of two states of conscious awareness in memory. Proceedings of the National Academy of Sciences, USA 94:5973-78. [APY]

Eacott, M. J. (1998) Acquisition and retention of visual discrimination learning after ablation of perirhinal cortex in the rat. Psychobiology 26:36-41. [MJE]

Eacott, M. J., Gaffan, D. \& Murray, E. A. (1994) Preserved recognition memory for small sets, and impaired stimulus identification for large sets, following rhinal cortex ablation in monkeys. European Journal of Neuroscience 6:146678. [rJPA, MJE]

Eacott, M. J. \& Heywood, C. A. (1995) Perception and memory: Action and interaction. Critical Reviews in Neurobiology 9:311-20. [MJE]

Eichenbaum, H. (1997) Declarative memory: Insights from cognitive neurobiology. Annual Review of Psychology 48:547-72. [SMZ]

Eichenbaum, H., Otto, T. \& Cohen, N. J. (1994) Two functional components of the hippocampal memory system. Behavioral and Brain Sciences 17:449-518. [aJPA, RDB, JCD-A, DLH, MPW, SMZ]

Eichenbaum, H., Stewart, C. \& Morris, R. G. M. (1990) Hippocampal representation in spatial learning. Journal of Neuroscience 10:331-39. [RDB]

Eifuku, S., Nishijo, H., Kita, T. \& Ono, T. (1995) Neuronal activity in the primate hippocampal formation during a conditional association task based on the subject's location. Journal of Neuroscience 15:4952-69. [aJPA, AT]

Ennaceur, A. \& Aggleton, J. P. (1994) Spontaneous recognition of object configurations in rats: Effects of fornix lesions. Experimental Brain Research 100:85-92. [aJPA]

(1997) The effects of neurotoxic lesions of the perirhinal cortex combined to fornix transection on object recognition memory in the rat. Behavioural Brain Research 88:181-93. [arJPA, DKB]

Ennaceur, A., Neave, N. \& Aggleton, J. P. (1996) Neurotoxic lesions of the perirhinal cortex do not mimic the behavioural effects of fornix transection in the rat. Behavioural Brain Research 80:9-25. [aJPA, DKB]

(1997) Spontaneous object recogntion and object location memory in rats: The effects of lesions in the cingulate cortices, the medial prefrontal cortex, the cingulum bundle and the fornix. Experimental Brain Research 113:509-519. [aJPA]

Eskandar, E. N., Richmond, B. J. \& Optican, L. M. (1992) Role of inferior temporal neurons in visual memory: I. Temporal encoding of information about visual images, recalled images, and behavioral context. Journal of Neurophysiology 68:1277-95. [aJPA]

Fahy, F. L., Riches, I. P. \& Brown, M. W. (1993a) Neuronal signals of importance to the performance of visual recognition memory tasks: Evidence from recordings of single neurones in the medial thalamus of primates. Progress in Brain Research 95:401-16. [arJPA]

(1993b) Neuronal activity related to visual recognition memory: Long-term memory and the encoding of recency and familiarity information in the primate anterior and medial inferior temporal and rhinal cortex. Experimental Brain Research 96:457-72. [arJPA, MJE]

Fazio, F., Perani, D., Gilardi, M. C., Colombo, F., Cappa, S. F., Vallar, G., Bettinardi, V., Paulesu, E., Alberoni, M., Bressi, S., Franceshci, M. \& Lenzi, G. L. (1992) Metabolic impairment in human amnesia: A PET study of memory networks. Journal of Cerebral Blood Flow and Metabolism 12:35358. [aJPA]

Foster, J. K., ed. (in press) Special edition of Memory on the theme of "Neuroimaging and memory." To be published in 1999. This will also be published in book form by Psychology Press (Taylor \& Francis). [JKF]

Foster, J. K., Ainsworth, J., Faratin, P. \& Shapiro, J. (1997) Investigating the mnemonic functions of the hippocampus: A computational neuroscience perspective. In: Cognitive models of memory, ed. M. Conway \& S. Gathercole. Psychology Press (Taylor \& Francis). [JKF]

Foster, J. K., Black, S. E., Buck, B. H. \& Bronskill, M. J. (1997) Executive and associated functions in normal and pathological aging: A neuroimaging perspective. In: Methodology of frontal and executive function, ed. P. Rabbitt. Psychology Press (Taylor \& Francis). [ JKF]

Foster, J. K., Lidder, P. \& Suenram, S. I. (1998) Glucose facilitation of memory performance in normal young participants: Fractionation of enhancement effects? Psychopharmacology 137:259-70. [JKF]

Foster, J. K. \& Rawlins, J. N. P. (1992a) The role of the hippocampus in the performance of a continuous non-matching task. Psychobiology 20:189-97. $[\mathrm{JKF}]$

(1992b) The effects of a hippocampal aspiration lesion upon the performance of a delayed alternation Go/No-Go task. Behavioural Brain Research 47:35-48. [JKF]

Foster, J. K., Rossell, S. L. \& Meudell, P. R. (in preparation) Temporal judgement: Judging what has been seen is preserved in schizophrenia, but not when. $[\mathrm{JKF}]$

Freed, D. M. \& Corkin, S. (1988) Rate of forgetting in H.M.: 6-month recognition. Behavioral Neuroscience 102:823-27. [aJPA]

Freed, D. M., Corkin, S. \& Cohen, N. J. (1987) Forgetting in H.M. - A second look. Neuropsychologia 25:461-72. [aJPA]

Freeman, J. H., Jr., Cuppernell, C., Flannery, K. \& Gabriel, M. (1996) Contextspecific multi-site cingulate cortical, limbic thalamic and hippocampal neuronal activity during concurrent discriminative approach and avoidance training in rabbits. Journal of Neuroscience 16:1538-49. [MG]

Freeman, J. H., Jr., Weible, A., Rossi, J. \& Gabriel, M. (1997) Entorhinal cortex lesions disrupt processing and alter amygdalar and cingulate cortical neuronal activity during extinction of discriminative avoidance learning. Experimental Brain Research 115:445-57. [MG]

Friedman, H. R., Janas, J. D. \& Goldman-Rakic, P. S. (1990) Enhancement of metabolic activity in the diencephalon of monkeys performing working memory tasks: A 2-deoxyglucose study in behaving rhesus monkeys. Journal of Cognitive Neuroscience 2:18-31. [aJPA]

Fulvi-Mari, C. \& Treves, A. (1998) Modeling neocortical areas with a modular neural network. Biosystems 48:47-55. [AT]

Fuster, J. M. (1989) The prefrontal cortex: Anatomy, physiology and neuropsychology of the frontal lobe. Raven Press. [JKF]

Fuster, J. M. \& Jervey, J. P. (1981) Inferotemporal neurons distinguish and retain behaviorally relevant features of visual stimuli. Science 212:952-55. [aJPA]

Gabriel, M. (1993) Discriminative avoidance learning: A model system. In: Neurobiology of cingulate cortex and limbic thalamus: A comprehensive handbook, ed. B. A. Vogt \& M. Gabriel. Birkhauser. [aJPA, JCD-A, MG]

Gabriel, M., Sparenborg, S. \& Stolar, N. (1987) Hippocampal control of cingulate cortical and anterior thalamic information processing during learning in rabbits. Experimental Brain Research 67:131-52. [MG]

Gabriel, M., Vogt, B. A., Kubota, Y., Poremba, A. \& Kang, E. (1991) Trainingstage related neuronal plasticity in limbic thalamus and cingulate cortex during learning: A possible key to mnemonic retrieval. Behavioural Brain Research 46:175-85. [MG]

Gabrieli, J., Brewer, J. B., Desmond, J. E. \& Glover, G. H. (1997) Separate neural bases of two fundamental memory processes in the human medial temporal lobe. Science 276:264-66. [aJPA]

Gaffan, D. (1974) Recognition impaired and association intact in the memory of monkeys after transection of the fornix. Journal of Comparative and Physiological Psychology 86:1100-1109. [aJPA]

(1991) Spatial organization of episodic memory. Hippocampus 1:262-64. [arJPA]

(1992a) The role of the hippocampus-fornix-mammillary system in episodic memory. In: Neuropsychology of memory (2nd edition), ed. L. R. Squire \& N. Butters. Guilford Press. [arJPA, MJE]

(1992b) Amnesia for complex naturalistic scenes and for objects following fornix transection in the rhesus monkey. European Journal of Neuroscience 4:38188. [arJPA]

(1994a) Dissociated effects of perirhinal cortex ablation, fornix transection and amygdalectomy: Evidence for multiple memory systems in the primate temporal lobe. Experimental Brain Research 99:411-22. [aJPA, DKB, MJE, SMZ]

(1994b) Scene-specific memory for objects: A model of episodic memory impairment in monkeys with fornix transection. Journal of Cognitive Neuroscience 6:305-20. [aJPA, AP]

(1998) Ideothetic input into object-place configuration as the contribution to memory of the monkey and human hippocampus. Experimental Brain Research 123:201-209. [AP]

Gaffan, D. \& Eacott, M. J. (1995) Uncinate fascicle section leaves delayed matching-to-sample intact, with both large and small sets. Experimental Brain Research 105:175-80. [arJPA]

Gaffan, D. \& Gaffan, E. A. (1991) Amnesia in man following transection of the fornix. Brain 114:2611-18. [arJPA]

Gaffan, D. \& Harrison, S. (1989) Place memory and scene memory: Effects of fornix transection in the monkey. Experimental Brain Research 74:202-12. [arJPA]

Gaffan, D. \& Lim, C. (1991) Hippocampus and the blood supply to TE: Parahippocampal pial section impairs visual discrimination learning in monkeys. Experimental Brain Research 87:227-31. [arJPA]

Gaffan, D. \& Murray, E. A. (1992) Monkeys with rhinal cortex lesions succeed in object discrimination learning despite 24 -hour intertrial intervals and fail at match to sample despite double sample presentations. Behavioral Neuroscience 106:30-38. [MJE] 
References/Aggleton \& Brown: Episodic memory, amnesia, and hippocampus

Gaffan, D. \& Parker, A. (1996) Interaction of perirhinal cortex with the fornixfimbria: Memory for objects and "object-in-place" memory. Journal of Neuroscience 16:5864-69. [aJPA]

Gaffan, D., Parker, A. \& Easton, A. (1998) Dense amnesia in macaques after section of anterior temporal stem, amygdala and fornix. Society for Neuroscience Abstracts 24:18. [AP]

Gaffan, D., Shields, C. \& Harrison, S. (1984) Delayed matching by fornixtransected monkeys: The sample, the push and the bait. Quarterly Journal of Experimental Psychology 36B:305-17. [arJPA]

Gaffan, E. A., Gaffan, D. \& Hodges, J. R. (1991) Amnesia following damage to the left fornix and to other sites. Brain 114:1297-313. [aJPA]

Garcia-Bengochea, F. \& Friedman, W. A. (1987) Persistent memory loss following section of the anterior fornix in humans. Surgical Neurology 27:361-64. [arJPA]

Gardiner, J. M. (1988) Functional aspects of recollective experience. Memory and Cognition 16:309-13. [aJPA]

Gardiner, J. M. \& Java, R. I. (1990) Recollective experience in word and nonword recognition. Memory and Cognition 19:617-23. [aJPA]

Gardiner J. M. \& Parkin, A. J. (1990) Attention and recollective experience in recognition memory. Memory and Cognition 18:579-83. [aJPA]

Geffen, G., Walsh, A., Simpson, D. \& Jeeves, M. (1980) Comparison of the effects of transcortical and transcallosal removal of intraventricular tumours. Brain 103:773-88. [aJPA]

Gentilini, M., De Renzi, E., Crisi, G. (1987) Bilateral paramedian thalamic artery infarcts: Report of eight cases. Journal of Neurology, Neurosurgery and Psychiatry 50:900-909. [aJPA]

Glanzer, M. \& Bowles, N. (1976) Analysis of the word frequency effect in recognition memory. Journal of Experimental Psychology: Human Learning and Memory 2:21-31. [LN]

Glees, P. \& Griffiths, H. B. (1952) Bilateral destruction of the hippocampus (Cornu ammonis) in a case of dementia. Psychiatric Neurological and Medical Psychology 123:193-204. [aJPA]

Glenn, M. J. \& Mumby, D. G. (1996) Place- and object-recognition deficits following lesions of the hippocampus or perirhinal cortex in rats: A double dissociation. Society for Neuroscience Abstracts 22:1120. [DGM] (1998) Place-memory is intact in rats with perirhinal cortex lesions. Behavioral Neuroscience 112:1353-65. [DGM]

Godden, D. R. \& Baddeley, A. D. (1975) Context-dependent memory in two natural environments: On land and underwater. British Journal of Psychology 66:325-31. [aJPA]

Goldenberg, G., Wimmer, A. \& Maly, J. (1983) Amnesic syndrome with a unilateral thalamic lesion: A case report. Journal of Neurology 229:79-86. [aJPA]

Gothard, K. M., Skaggs, W. E. \& McNaughton, B. L. (1996) Dynamics of mismatch correction in the hippocampal ensemble code for space: Interaction between path integration and environmental cues. Journal of Neuroscience 16:8027-640. [aJPA, AT]

Goulet, S., Douré, F. Y. \& Murray, E. A. (1998) Aspiration lesions of the amygdala disrupt the rhinal corticothalamic projection system in rhesus monkeys. Experimental Brain Research 119:131-40. [rJPA, AP]

Graf, P. \& Ryan, L. (1990) Transfer appropriate processing for implicit and explicit memory. Journal of Experimental Psychology: Learning, Memory, and Cognition 16:978-92. [LN]

Graf, P. \& Schacter, D. L. (1985) Implicit and explicit memory for new associations in normal and amnesic subjects. Journal of Experimental Psychology: Learning, Memory and Cognition 11:501-18. [DGM]

Graff-Radford, N. R., Tranel, D., van Hoesen, G. W. \& Brandt, J. P. (1990) Diencephalic amnesia. Brain 113:1-25. [aJPA, MPW]

Graham, K. S., Becker, J. T. \& Hodges, J. R. (1997) On the relationship between knowledge and memory for pictures: Evidence from the study of patients with semantic dementia and Alzheimer's disease. Journal of the International Neuropsychological Society 3:534-44. [KSG]

Graham, K. S. \& Hodges, J. R. (1997) Differentiating the roles of the hippocampal complex and the neocortex in long term memory storage: Evidence from the study of semantic dementia and Alzheimer's disease. Neuropsychology 11:7789. [aJPA, KSG, BJK]

Graham, K. S., Pratt, K. H. \& Hodges, J. R. (in press) A reverse temporal gradient for public events in a single case of semantic dementia. Neurocase. [KSG]

Graham, K. S., Simons, J. S., Pratt, K. H., Patterson, K. \& Hodges, J. R. (submitted) Insights from semantic dementia on the relationship between episodic and semantic memory. Neuropsychologia. [KSG]

Granon, S., Vidal, C., Thinus-Blanc, C., Changeus, J-P. \& Poucet, B. (1994) Working memory, response selection, and effortful processing in rats with medial prefrontal lesions. Behavioral Neuroscience 108:883-91. [aJPA]

Gray, J. A. (1982) The neuropsychology of anxiety: An enquiry into the functions of the septohippocampal system. Oxford University Press. [JKF, ADP]

Greene, E. \& Naranjo, J. N. (1986) Thalamic role in spatial memory. Behavioral Brain Research 19:123-31. [aJPA, JCD-A]
Gregg, V. H. \& Gardiner, J. M. (1994) Recognition memory and awareness: A large effect of study-test modalities on "Know" responses following a highly perceptual orienting task. European Journal of Cognitive Psychology 6:13147. [BJK]

Groenewegen, H. J. (1988) Organization of the afferent connections of the mediodorsal thalamic nucleus in the rat, related to the mediodorsalprefrontal topography. Neuroscience 24:379-431. [RGM]

Gronlund, S. D., Edwards, M. B. \& Ohrt, D. D. (1997) Comparison of the retrieval of item versus spatial position information. Journal of Experimental Psychology: Learning, Memory, and Cognition 23:1261-74. [DLH]

Gudden, H. (1896) Klinische und anatommische Beitrage zur Kenntnis der multiplen Alkoholneuritis nebst Bemerzungen uber die Regenerationsvorgange im peripheren Nervensystem. Archiv Psychiatrie 28:643-741. [aJPA]

Habib, M. \& Sirigu, A. (1987) Pure topographical disorientation: A definition and anatomical basis. Cortex 23:73-85. [aJPA]

Haist, F., Shimamura, A. P. \& Squire, L. R. (1992) On the relationship between recall and recognition memory. Journal of Experimental Psychology 18:691702. [aJPA]

Hamann, S. B. \& Squire, L. R. (1997) Intact perceptual memory in the absence of conscious memory. Behavioral Neuroscience 111(4):850-54. [aJPA]

Hanley, J. R., Davies, A. D. M., Downes, J. J. \& Mayes, A. R. (1994) Impaired recall of verbal material following rupture and repair of an anterior communicating artery aneurysm. Cognitive Neuropsychology 11:543-78. [aJPA]

Harasty, J. A., Halliday, G. M., Code, C. \& Brooks, W. S. (1996) Quantification of cortical atrophy in a case of progressive fluent aphasia. Brain 119:181-90. $[\mathrm{KSG}]$

Hart, M., Poremba, A. \& Gabriel, M. (1997) The nomadic engram: Overtraining eliminates the impairment of discriminative avoidance behavior produced by limbic thalamic lesions. Behavioural Brain Research 82:169-77. [MG]

Hasselmo, M. E. \& Wyble, B. P. (1997) Free recall and recognition in a network model of the hippocampus: Simulating effects of scopolamine on human memory functions. Behavioural Brain Research 89:1-34. [ARM]

Hassler, F. (1962) New aspects of brain functions revealed in brain diseases. In: Frontiers in brain research, ed. J. D. French. Columbia University Press. [aJPA]

Heilman, K. M., Bowers, D., Watson, R. T., Day, A., Valenstein, E., Hammond, E. \& Duara, R. (1990) Frontal hypermetabolism and thalamic hypometabolism in a patient with abnormal orienting and retrosplenial amnesia. Neuropsychologia 28:161-69. [aJPA]

Heilman, K. M. \& Sypert, G. W. (1977) Korsakoff's syndrome resulting from bilateral fornix lesions. Neurology 27:490-93. [aJPA]

Herdegen, T. (1996) Jun, Fos, and CREB/ATF transcription factors in the brain: Control of gene expression under normal and pathophysiological conditions. Neuroscientist 2:153-61. [aJPA]

Higuchi, S. I. \& Miyashita, Y. (1996) Formation of mnemonic neuronal responses to visual paired associates in inferotemporal cortex is impaired by perirhinal and entorhinal lesions. Proceedings of the National Academy of Sciences, USA 93:739-43. [aJPA]

Hintzmann, D. L. \& Caulton, D. A. (1997) Recognition memory and modality judgements: A comparison of retrieval dynamics. Journal of Memory and Language 37:1-23. [DLH]

Hintzmann, D. L., Caulton, D. A. \& Levitin, D. J. (1998) Retrieval dynamics in recognition and list discrimination: Further evidence of separate processes of familiarity and recall. Memory and Cognition 26:449-62. [DLH]

Hintzmann, D. L. \& Curran, T. (1994) Retrieval dynamics of recognition and frequency judgments: Evidence for separate processes of familiarity and recall. Journal of Memory and Language 33:1-18. [DLH]

(1997) Comparing retrieval dynamics in recognition memory and lexical decision. Journal of Experimental Psychology: General 126:228-47. [DLH]

Hirshman, E. \& Master, S. (1997) Modeling the conscious correlates of recognition memory: Reflections on the remember-know paradigm. Memory and Cognition 25:345-51. [aJPA]

Hirst, W., Johnson, M. K., Kim, J. K., Phelps, E. A., Risse, G. \& Volpe, B. T. (1986) Recognition and recall in amnesics. Journal of Experimental Psychology 12:445-51. [aJPA]

Hirst, W., Johnson, M. K., Phelps, E. A. \& Volpe, B. T. (1988) More on recognition and recall in amnesics. Journal of Experimental Psychology 14:758-62. [aJPA]

Hodges, J. R. \& Carpenter, K. (1991) Anterograde amnesia with fornix damage following removal of IIIrd ventricle colloid cyst. Journal of Neurology, Neurosurgery and Psychiatry 54:633-38. [aJPA]

Hodges, J. R. \& Graham, K. S. (1998) A reversal of the temporal gradient for famous person knowledge in semantic dementia: Implications for the neural organisation of long-term memory. Neuropsychologia 36:803-25. [KSG]

Hodges, J. R. \& McCarthy, R. A. (1993) Autobographical amnesia resulting from bilateral paramedian thalamic infarction. Brain 116:921-40. [aJPA] 
References/Aggleton \& Brown: Episodic memory, amnesia, and hippocampus

Hodges, J. R. \& Patterson, K. (1996) Nonfluent progressive aphasia and semantic dementia: A comparative neuropsychological study. Journal of the International Neuropsychological Society 2:511-24. [KSG]

Hodges, J. R., Patterson, K., Oxbury, S. \& Funnell, E. (1992) Semantic dementia: Progressive fluent aphasia with temporal lobe atrophy. Brain 115:1783-806. [aJPA, KSG]

Holdstock, J. S., Shaw, C. \& Aggleton, J. P. (1995) The performance of amnesic subjects on tests of delayed matching-to-sample and delayed matching-toposition. Neuropsychologia 33:1583-96. [arJPA]

Holmes, E. J. \& Butters, M. S. (1983) An examination of the effects of mammillary-body lesions on reversal learning sets in monkeys. Physiological Psychology 3:159-65. [aJPA]

Honey, R. C., Watt, A. \& Good, M. (1998) Hippocampal lesions disrupt an associative-mismatch process. Journal of Neuroscience 18:2226-30. [aJPA, $\mathrm{LN}, \mathrm{ADP}]$

Horel, J. A., Pytko-Joiner, D.Voytko, M. L. \& Salsbury, K. (1987) The performance of visual tasks while segments of the inferotemporal cortex are suppressed by cold. Behavioural Brain Research 23:29-42. [MJE]

Horel, J. A., Voytko, M. L. \& Salsbury, K. G. (1984) Visual learning suppressed by cooling the temporal pole. Behavioral Neuroscience 98:310-24. [MJE]

Hull, C. L. (1943) Principles of behavior. Appleton-Century-Crofts. [EEK]

Hunt, P. R. \& Aggleton, J. P. (1991) Medial dorsal thalamic lesions and working memory in the rat. Behavioural and Neural Biology 55:227-46. [aJPA, JCD-A]

(1998) Neurotoxic lesions of the dorsomedial thalamus impair the acquisition but not the performance of delayed matching to place by rats: A deficit in shifting response rules. Journal of Neuroscience 97:129-41. [rJPA]

Huppert, F. A. \& Piercy, M. (1978) The role of trace strength in recency and frequency judgments by amnesic and control subjects. Quarterly Journal of Experimental Psychology 30:347-54. [aJPA]

(1979) Normal and abnormal forgetting in organic amnesia: Effect of locus of lesion. Cortex 15:385-90. [aJPA]

Insausti, R., Amaral, D. G. \& Cowan, W. M. (1987) The entorhinal cortex of the monkey: II. Cortical afferents. Journal of Comparative Neurology 264:35695. [aJPA]

Jackson-Smith, P., Kesner, R. P. \& Chiba, A. A. (1993) Continuous recognition of spatial and nonspatial stimuli in hippocampal-lesioned rats. Behavioral and Neural Biology 59:107-19. [RPK]

Jacoby, L. L. (1983) Remembering the data: Analyzing interactive process in reading. Journal of Verbal Learning and Verbal Behavior 22:485-508. [LN]

(1991) A process dissociation framework: Separating automatic from intentional uses of memory. Journal of Memory and Language 30:513-41. [aJPA, ADP, APY]

Jacoby, L. L. \& Dallas, M. (1981) On the relationship between autobiographical memory and perceptual learning. Journal of Experimental Psychology: General 3:306-40. [aJPA]

Jarrard, L. E. (1995) What does the hippocampus really do? Behavioural Brain Research 71:1-10. [DKB]

Jones, B. \& Mishkin, M. (1972) Limbic lesions and the problem of stimulusreinforcement associations. Experimental Neurology 36:362-77. [aJPA]

Jones, E. G. (1985) The thalamus. Plenum Press. [RGM]

Jones, G. V. (1987) Independence and exclusivity among psychological processes: Implications for the structure of recall. Psychological Review 94:229-35. [aJPA]

Jones, G. V. \& Gardiner, J. M. (1990) Recognition failure when recognition targets and recall cues are identical. Bulletin of the Psychonomic Society 28:105-108. [aJPA]

Kang, E. \& Gabriel, M. (1998) Hippocampal modulation of cingulo-thalamic neuronal activity and discriminative avoidance learning rabbits. Hippocampus 8:491-510. [MG]

Kapur, N., Crewes, H., Wise, R., Abbott, P., Carter, M., Millar, J. \& Lang, D. (1999) Mammillary body damage results in memory impairment but not amnesia. Neurocase (in press). [NK]

Kapur, N., Ellison, D., Parkin, A. J., Hunkin, N., Burrows, E., Sampson, S. A. \& Morrison, E. A. (1994) Bilateral temporal lobe pathology with sparing of medial temporal lobe structures: Lesion profile and pattern of memory disorder. Neuropsychologia 32:1-16. [aJPA, KSG]

Kapur, N., Ellison, D., Smith, M. P., McLellan, D. L. \& Burrows, E. H. (1992) Focal retrograde amnesia following bilateral temporal lobe pathology: A neuropsychological and magnetic resonance study. Brain 115:73-85. [aJPA KSG]

Kapur, N., Scholey, K., Moore, E., Barker, S., Brice, J., Mayes, A. \& Fleming, J. (1995) The mammillary bodies revisited: Their role in human memory functioning. In: Neuropsychological explorations in memory and cognition: Essays in honor of Nelson Butters, ed. L. Cermak. Plenum Press. [aJPA, NK]

Kartsounis, L. D., Rudge, P. \& Stevens, J. M. (1995) Bilateral lesions of CAl and CA2 fields of the hippocampus are sufficient to cause a severe amnesic syndrome in humans. Journal of Neurology, Neurosurgery and Psychiatry 59:95-98. [aJPA]

Kesner, R. P. (1998) Neurobiological views of memory. In: Neurobiology of learning and memory, ed. J. Martinez \& R. Kesner. Academic Press. [RPK]

Kesner, R. P., Ravindranath, A., Jackson-Smith, P., Giles, R. \& Chiba, A. A (submitted) A neural circuit analysis of visual object working memory: Role of perirhinal, entorhinal and TE2 cortex. [RPK]

Kievit, J. \& Kuypers, H. G. J. M. (1977) Organization of the thalamo-cortical connexions to the frontal lobe in the rhesus monkey. Experimental Brain Research 29:299-322. [aJPA]

Kim, J. J. \& Fanselow, M. S. (1992) Modality-specific retrograde amnesia of fear. Science 256:675-77. [aJPA]

Knight, R.T. (1996) Contribution of human hippocampal region to novelty detection. Nature 383:256-59. [aJPA]

Knoth, R. L. \& Mair, R. G. (1991) Response latency and accuracy on a pretrained nonmatch to sample task in rats recovered from pyrithiamine-induced thiamin deficiency. Behavioral Neuroscience 105:375-85. [RGM]

Knowlton, B. J. (1998) The relationship between remembering and knowing: A cognitive neuroscience perspective. Acta Psychologica 98:253-65. [BJK]

Knowlton, B. J. \& Squire, L. R. (1995) Remembering and knowing: Two different expressions of declarative memory. Journal of Experimental Psychology 21(3):699-710. [arJPA, BJK, APY, SMZ]

Koehler, S., Black, S. E., Sinden, M., Kidron, D., Szekely, C., Parker, J., Foster, J. K., Moscovitch, M., Winocur, G., Szalai, J. P. \& Bronskill, M. (1998) Memory impairments in relation to hippocampal and parahippocampal atrophy in Alzheimer's disease: An MR volumetry study. Neuropsychologia 36:101-14. [JKF]

Koger, S. M. \& Mair, R. G. (1994) Comparison of the effects of frontal cortical and thalamic lesions on measures of olfactory learning and memory in the rat. Behavioral Neuroscience 108:1088-100. [RGM]

Kolb, B., Pittman, K., Sutherland, R. J. \& Whishaw, I. Q. (1982) Dissociation of the contributions of the prefrontal cortex and dorsomedial thalamic nucleus to spatially guided behavior in the rat. Behavioural Brain Research 6:365-78 [aJPA]

Kopelman, M. D. (1995) The Korsakoff's syndrome. British Journal of Psychiatry 166:154-73. [JCD-A]

Kosel, K. C., Van Hoesen, G. W. \& Rosene, D. (1983) A direct projection from the perirhinal cortex (area 35) to the subiculum in the rat. Brain Research 269:347-51. [RDB]

Krieckhaus, E. E. (1964) Decrements in avoidance behavior following mammillothalamic tractotomy in cats. Journal of Neurophysiology 27:753-67. [EEK]

(1998) Papez circuit inhibits stress and alarm and initiates voluntary action. Behavioral and Brain Sciences (submitted). [EEK]

Krieckhaus, E. E., Donahoe, J. W. \& Morgan, M. A. (1992) Paranoid schizophrenia may be caused by dopamine hyperactivity of CAl hippocampus. Journal of Biological Psychiatry 31:560-70. [EEK]

Kritchevsky, M., Graff-Radford, N. R. \& Damasio, A. R. (1987) Normal memory after damage to medial thalamus. Archives of Neurology 44:959-64. [arJPA, NK, MPW]

Kroll, N. E. A., Knight, R. T., Metcalfe, J., Wolf, E. S. \& Tulving, E. (1996) Cohesion failure as a source of memory illusions. Journal of Memory and Language 35:176-96. [APY]

Langlais, P. J. \& Savage, L. M. (1995) Thiamine deficiency in rats produces cognitive and memory deficits on spatial tasks that correlate with tissue loss in diencephalon, cortex and white matter. Behavioural Brain Research 68:7589. [aJPA, JCD-A, RGM]

Langlais, P. J., Zhang, S.-X. \& Savage, L. M. (1996) Neuropathology of thiamine deficiency: An update on the comparative analysis of human disorders and experimental models. Metabolic Brain Disease 11:19-37. [RGM]

Lauro-Grotto, R., Piccini, C., Borgo, F. \& Treves, A. (1997) What remains of memories lost in Alzheimer and herpetic encephalitis. Society for Neuroscience Abstracts 23:1889. [AT]

Leng, N. R. C. \& Parkin., A. J. (1989) Aetiological variation in the amnesic syndrome: Comparisons using the Brown-Peterson task. Cortex 25:251-59. [aJPA]

Leonard, B. W., Amaral, D. G., Squire, L. R. \& Zola-Morgan, S. (1995) Transient memory impairment in monkeys with bilateral lesions of the entorhinal cortex. Journal of Neuroscience 15:5637-59. [aJPA]

Levasseur, M., Baron, J. C., Sette, G., LeGault-Demare, F., Pappata, S., Mauguiere, F., Benoit, N., Tran Dinh, S., Degos, J. D., Laplane, D. \& Mazoyer, B. (1992) Brain energy metabolism in bilateral paramedian thalamic infarcts. Brain 115:795-807. [aJPA]

Li, L., Miller, E. K. \& Desimone, R. (1993) The representation of stimulus familiarity in anterior inferior temporal cortex. Journal of Neurophysiology 69:1918-29. [aJPA, MJE]

Lindeboom, J. (1989) The assessment of anterograde amnesia. Journal of Clinical and Experimental Neuropsychology 11:345. [MPW] 
References/Aggleton \& Brown: Episodic memory, amnesia, and hippocampus

Liu, P. (1998) Perirhinal cortex contributions to spatial memory. Ph. D. thesis, University of Otago, Dunedin, New Zealand. [JCD-A]

Liu, P. \& Bilkey, D. K. (1998a) Excitotoxic lesions centered on perirhinal cortex produce delay-dependent deficits in a test of spatial memory. Behavioral Neuroscience 112:512-24. [DKB]

(1998b) Lesions of perirhinal cortex produce spatial memory deficits in the radial maze. Hippocampus 8:114-21. [DKB, JCD-A, DGM]

(1998c) Perirhinal cortex contributions to performance in the Morris water maze. Behavioral Neuroscience 112:304-15. [DKB, DGM]

Lorente de No, R. (1934) Studies on the structure of the cerebral cortex. II. Continuation of the study of the ammonic system. Journal für Psychologie und Neurologie 46:113-77. [aJPA]

Lueschow, A., Miller, E. K. \& Desimone, R. (1994) Inferior temporal mechanisms for invariant object recognition. Cerebral Cortex 4:523-31. [aJPA]

Maguire, E. A., Frackowiak, R. S. J. \& Frith, C. D.(1996) Learning to find your way: A role for the human hippocampal formation. Proceedings of the Royal Society of London B 263:1745-50. [aJPA]

Mahut, H. (1971) Spatial and object reversal learning in monkeys with partial temporal lobe ablations. Neuropsychologia 9:409-24. [aJPA]

(1972) A selective spatial deficit in monkeys after transection of the fornix. Neuropsychologia 10:65-74. [aJPA]

Mair, R. G. (1994) On the role of thalamic pathology in diencephalic amnesia. Reviews in the Neurosciences 5:105-40. [RGM]

Mair, R. G., Anderson, C. D., Langlais, P. J. \& McEntee, W. J. (1988) Behavioral impairments, brain lesions and monoaminergic activity in the rat following recovery from a bout of thiamin deficiency. Behavioural Brain Research 27:223-39. [RGM]

Mair, R. G., Burk, J. A. \& Porter, M. C. (1998) Lesions of the frontal cortex, hippocampus, and intralaminar thalamic nuclei have distinct effects on remembering. Behavioral Neuroscience 112:772-92. [RGM]

Mair, W. G. P., Warrington, E. K. \& Weiskrantz, L. (1979) Memory disorders in Korsakoff's psychosis. A neuropathological and neuropsychological investigation of two cases. Brain 102:749-83. [aJPA, RGM]

Malamud, N. \& Skillicorn, S. A. (1956) Relationship between the Wernicke and the Korsakoff syndrome. American Medical Association Archives of Neurology and Psychiatry 76:585-96. [RGM]

Malamut, B. L., Graff-Radford, N., Chawluk, J., Grossman, R. I. \& Gur, R. C. (1992) Memory in a case of bilateral thalamic infarction. Neurology 42:16369. [aJPA]

Malkova, L. \& Mishkin, M. (1997) Memory for the location of objects after separate lesions of the hippocampus and parahippocampal cortex in rhesus monkeys. Society for Neuroscience Abstracts 23:12. [SMZ]

Mandler, G. (1980) Recognizing: The judgment of previous occurrence. Psychological Review 87:252-71. [aJPA, LN, APY]

Mark, V. H., Barry, H., Mclardy, T. \& Ervin, F. R. (1970) The destruction of both anterior thalamic nuclei in a patient with intractable agitated depression. Journal of Nervous and Mental Disorders 150:266-72. [aJPA]

Markowitsch, H. J. (1982) Thalamic mediodorsal nucleus and memory: A critical evaluation of studies in animals and man. Neuroscience and Biobehavioral Reviews 6:351-80. [aJPA, RGM, HJM]

(1988) Diencephalic amnesia: A reorientation towards tracts? Brain Research Reviews 13:351-70. [aJPA, HJM]

(1992) Diencephalic amnesia. In: Trastornos de la memoria, ed. D. Barcia Salorio. Editorial MCR. [HJM]

(1994) Effects of emotion and arousal on memory processing by the brain. In: Memory, learning and the brain, ed. J. Delacour. World Scientific. [HJM]

(1999) The limbic system. In: The MIT encyclopedia of cognitive science, ed. R. Wilson \& F. Keil. MIT Press. [HJM]

Markowitsch, H. J., Calabrese, P., Haupts, M., Durwen, H. F., Liess, J. \& Gehlen, W. (1993) Searching for the anatomical basis of retrograde amnesia. Journal of Clinical and Experimental Neuropsychology 15:947-76. [aJPA]

Markowitsch, H. J., Emmans, D., Irle, E., Streicher, M. \& Preilowski, B. (1985) Cortical and subcortical afferent connections of the primate's temporal pole: A study of rhesus monkeys, squirrel monkeys, and marmosets. Journal of Comparative Neurology 242:425-58. [arJPA]

Markowitsch, H. J., von Cramon, D. Y. \& Schuri, U. (1993) Mnestic performance profile of a bilateral diencephalic infarct patient with preserved intelligence and severe amnesic disturbances. Journal of Clinical and Experimental Neuropsychology 15:627-52. [HJM]

Markowitsch, H. J., Weber-Luxemburger, G., Ewald, K., Kessler, J. \& Heiss, W.D. (1997) Patients with heart attacks are not valid models for medial temporal lobe amnesia. A neuropsychological and FDG-PET study with consequences for memory research. European Journal of Neurology 4:178-84. [aJPA, HJM]

Markowska, A. L., Olton, D. S., Murray, E. A. \& Gaffan, D. (1989) A comparative analysis of the role of the fornix and cingulate cortex in memory: Rats. Experimental Brain Research 74:187-201. [aJPA]

Martin, K. M. \& Aggleton, J. P. (1993) Contextual effects on the ability of divers to use decompression tables. Applied Cognitive Psychology 7:311-16. [aJPA]
Mayes, A. R. \& Downes, J. J. (1997) What do theories of the functional deficits(s) underlying amnesia have to explain? Memory 5:3-36. [aJPA, JKF, ADP] Also published as Chapter 1 of Theories of organic amnesia, ed. A. R. Mayes \& J. J. Downes. Psychology Press (Taylor \& Francis). [JKF]

Mayes, A. R., Meudell, P. R., Mann, D. \& Pickering, A. (1988) Location of lesions in Korsakoff's syndrome: Neuropsycholgical and neuropathological data on two patients. Cortex 24:367-88. [aJPA]

McClelland, J. L., McNaughton, B. L. \& O'Reilly, R. C. (1995) Why there are two complementary learning systems in the hippocampus and neocortex: Insights from the successes and failures of connectionist models of learning and memory. Psychological Review 102:419-57. [LN]

McKee, R. D. \& Squire, L. R. (1992) Equivalent forgetting rates in long-term memory for diencephalic and medial temporal lobe amnesia. Journal of Neuroscience 12:3765-72. [aJPA]

McMackin, D., Cockburn, J., Anslow, P. \& Gaffan, D. (1995) Correlation of fornix damage with memory impairment in six cases of colloid cyst removal. Acta Neurochirugica 135:12-18. [arjPA]

McNaughton, B. L., Barnes, C. A., Gerrard, J. L., Gothard, K., Jung, M. W., Knierin, J. J., Kudrimoiti, Q. Y., Skaggs, W. E., Suster, M. \& Weaver, K. L. (1996) Deciphering the hippocampal polyglot: The hippocampus as a path integration system. Journal of Experimental Biology 199:173-85. [AP]

Mennemeier, M., Fennell, E., Valenstein, E. \& Heilman, K. M. (1992) Contributions of the left intralaminar and medial thalamic nuclei to memory: Comparisons and a case report. Archives of Neurology 49:1050-58. [RGM]

Meunier, M., Bachevalier, J., Mishkin, M. \& Murray, E. A. (1993) Effects on visual recognition of combined and separate ablations of the entorhinal and perirhinal cortex in rhesus monkeys. Journal of Neuroscience 12:5418-32. [aJPA, MJE]

Meunier, M., Hadfield, J., Bachevalier, J. \& Murray, E. A. (1996) Effects of rhinal cortex lesions combined with hippocampectomy on visual recognition memory in rhesus monkeys. Journal of Neurophysiology 75:1190-205. [aJPA]

Miller, E. K. \& Desimone, R. (1994) Parallel neuronal mechanisms for short-term memory. Science 263:520-22. [aJPA]

Miller, E. K., Li, L. \& Desimone, R. (1993) Activity of neurons in anterior inferior temporal cortex during a short-term memory task. Journal of Neuroscience 13:1460-78. [aJPA, DLH]

Miller, L. A., Munoz, D. G. \& Finmore, M. (1993) Hippocampal sclerosis and human memory. Archives of Neurology 50:391-94. [aJPA]

Mirsky, R., Adolphs, R., Volkov, I., Bechara, A., Damasio, H. \& Howard, M. A., III. (1997) Single-unit neuronal activity in human amygdala and ventral frontal cortex recorded during emotional experience. Society for Neuroscience Abstracts 23:1318. [AT]

Mishkin, M. (1978) Memory in monkeys severely impaired by combined, but not by separate removal of amygdala and hippocampus. Nature 273:297-98. [aJPA]

Mishkin, M. \& Delacour, J. D. (1975) An analysis of short-term visual memory in the monkey. Journal of Experimental Psychology: Animal Behavior Processes $1: 326-34 . \quad[\mathrm{rJPA}]$

Mishkin, M., Malamut, B. \& Bachevalier, J. (1984) Memories and habits: Two neural systems. In: Neurobiology of learning and memory, ed. G. Lynch, J. L. McGaugh \& N. M. Weinberger. Guilford Press. [RDB]

Mishkin, M. \& Murray, E. M. (1994) Stimulus recognition. Current Opinion in Neurobiology 4:200-206. [aJPA, SMZ]

Miyashita, Y., Okuno, H., Tokuyama, W., Ihara, T. \& Nakajima, K. (1996) Feedback signal from medial temporal lobe mediates visual associative mnemonic codes of inferotemporal neurons. Cognitive Brain Research 5:81-86. [aJPA]

Miyashita, Y., Rolls, E. T., Cahusac, P. M. B., Niki, H. \& Feigenbaum, J. D. (1989) Activity of hippocampal formation neurons in the monkey related to a conditional spatial response task. Journal of Neurophysiology 61:669-77. [aJPA, AT]

Mizumori, S. J. Y., Miya, D. Y. \& Ward, K. E. (1994) Reversible inactivation of the lateral dorsal thalamus disrupts hippocampal place representation and impairs spatial learning. Brain Research 644:168-74. [aJPA, JCD-A]

Mizumori, S. J. Y. \& Williams, J. D. (1993) Directionally sensitive mnemonic properties of neurons in the lateral dorsal nucleus of the thalamus in rats. Journal of Neuroscience 13:4015-28. [aJPA]

Mori, E., Yamadori, A. and Mitani, Y (1986) Left thalamic infarction and disturbance of verbal memory: A clinicoanatomical study with a new method of computed tomographic stereotaxic lesion localization. Annals of Neurology 20:671-76. [aJPA]

Morris, R. G. M., Garrud, P., Rawlins, J. N. P. \& O'Keefe, J. (1982) Place navigation impaired in rats with hippocampal lesions. Nature 297:681-83. [aJPA, RDB]

Moscovitch, M. \& Nadel, L. (1998) Consolidation revisited: In defense of the multiple-trace model. Current Opinion in Neurobiology 8:297-300. [LN]

Moser, E., Moser, M. \& Anderson, P. (1993) Spatial learning impairment parallels the magnitude of dorsal hippocampal lesions, but is hardly present following ventral lesions. Journal of Neuroscience 13:3916-25. [SMZ] 
Moser, M.-B., Moser, E. I., Forrest, E., Andersen, P. \& Morris, R. G. M. (1995) Spatial learning with a minislab in the dorsal hippocampus. Proceedings of the National Academy of Sciences USA 92:9697-701. [rJPA, SMZ]

Muller, R. U., Ranck, J. B., Jr. \& Taube, J. S. (1996) Head direction cells: Properties and functional significance. Current Opinions in Neurobiology 6:196-206. [JB]

Mulligan, N. \& Hirshman, E. (1995) Speed-accuracy trade-offs and the dual process model of recognition memory. Journal of Memory and Language 34:1-18. [DLH]

Mumby, D. G. \& Pinel, J. P. J. (1994) Rhinal cortex lesions and object recognition in rats. Behavioral Neuroscience 108:11-18. [aJPA, MJE, DGM]

Mumby, D. G., Pinel, J. P. J. \& Dastur, F. N. (1993) Mediodorsal thalamic lesions and object recognition in rats. Psychobiology 21:27-36. [aJPA]

Mumby, D. G., Pinel, J. P. J., Kornecook, T. J., Shen, M. J. \& Redila, V. A. (1995) Memory deficits following lesions of hippocampus or amygdala in rat: Assessment by an object-memory test battery. Psychobiology 23:26-36. [rJPA, SMZ]

Mumby, D. G., Wood, E. R., Duva, C. A., Kornecook, T. J., Pinel, P. J. \& Phillips, A. G. (1996) Ischemia-induced object-recognition deficits in rats are attenuated by hippocampal ablation before or soon after ischemia. Behavioral Neuroscience 110:266-81. [aJPA, DGM]

Mumby, D. G., Wood, E. R. \& Pinel, J. P. J. (1992) Object recognition memory in rats is only mildly impaired by lesions of the hippocampus and amygdala. Psychobiology 20:18-27. [DGM]

Mummery, C. J., Patterson, K., Wise, R. J. S., Price, C. J. \& Hodges, J. R. (in press) Disrupted temporal lobe connections in semantic dementia. Brain. [KSG]

Murray, D. J. (in press) Language and psychology: 19th-century developments outside Germany: A survey. In: History of the language sciences: An international handbook on the evolution of the study of language, ed. S Auroux, K. Koerner, H.-J. Niederehe \& K. Versteegh. De Gruyter. [DJM]

Murray, E. A. (1996) What have ablation studies told us about the neural substrates of stimulus memory? Seminars in the Neurosciences 8:13-22. [aJPA, DBK, $\mathrm{MJE}]$

Murray, E. A., Davidson, M., Gaffan, D., Olton, D. S. \& Suomi, S. (1989) Effects of fornix transection and cingulate coritical ablation on spatial memory in rhesus monkeys. Experimental Brain Research 74:173-86. [aJPA]

Murray, E. A., Gaffan, D. \& Mishkin, M. (1993) Neural substrates of visual stimulus-stimulus association in rhesus monkeys. Journal of Neuroscience 13:4549-61. [aJPA, MJE]

Murray, E. A. \& Mishkin, M. (1986) Visual recognition in monkeys following rhinal cortical ablations combined with either amygdalectomy or hippocampectomy. Journal of Neuroscience 7:1991-2003. [aJPA]

(1996) Forty-minute visual recognition memory in rhesus monkeys with hippocampal lesions. Society for Neuroscience Abstracts 22:281, 116.9. [aJPA, DJM]

(1998) Object recognition and location memory in monkeys with excitotoxic lesions of the amygdala and hippocampus. Journal of Neuroscience 18:656882. [rJPA, SMZ]

Murray, E. A. \& Wise, S. P. (1996) Role of the hippocampus plus subjacent cortex but not amygdala in visuomotor conditional learning in rhesus monkeys. Behavioral Neuroscience 110:1261-70. [AP]

(1997) Role of orbitoventral prefrontal cortex in conditional motor learning Society for Neuroscience Abstracts 23:11. [AP]

Naber, P. A., Cabellero-Bleda, M., Jorritsma-Byham, B. \& Witter, M. (1997) Parallel inputs to the hippocampal memory system through the peri- and postrhinal cortices. Neuroreport 8(11):2617-21. [aJPA, RDB]

Naber, P. A., Witter, M. P. \& Lopes da Silva, F. H. (submitted) Perirhinal cortex input to the hippocampal formation in the rat: Evidence for parallel pathways, both direct and indirect. A combined physiological and anatomical study. European Journal of Neuroscience. [MPW]

Nadel, L. \& Moscovitch, M. (1997) Consolidation, retrograde amnesia and the hippocampal formation. Current Opinion in Neurobiology 7:217-27. [JKF, LN]

(1998) Hippocampal contributions to cortical plasticity. Neuropharmacology 37:431-39. [LN]

Neave, N., Lloyd, S., Sahgal, A. \& Aggleton, J. P. (1994) Lack of effect of lesions in the anterior cingulate cortex and retrosplenial cortex on certain tests of spatial memory in the rat. Behavioural Brain Research 65:89-101. [aJPA]

Neave, N., Nagle, S. \& Aggleton, J. P. (1997) Evidence for the involvement of the mamillary bodies and cingulum bundle in allocentric spatial processing by rats. European Journal Neuroscience 9:101-15. [aJPA]

Neave, N., Nagle, S., Sahgal, A. \& Aggleton, J. P. (1996) The effects of discrete cingulum bundle lesions in the rat on the acquisition and performance of two tests of spatial working memory. Behavioural Brain Research 80:75-85. [aJPA, SMZ]

Nunn, J. \& Hodges, H. (1994) Cognitive deficits induced by global cerebral ischaemia: Relationship to brain damage and reversal by transplants. Behavioural Brain Research 65:1-31. [arJPA]
O’Boyle, V. J., Murray, E. A. \& Mishkin, M. (1993) Effects of excitotoxic amygdalohippocampal lesions on visual recognition in rhesus monkeys. Society for Neuroscience Abstracts 19:438, 186.4. [aJPA]

O'Keefe, J. (1993) Hippocampus, theta rhythms and spatial memory. Current Opinion in Neurobiology 3:917-24. [aJPA]

O'Keefe, J. \& Burgess, N. (1996) Geometric determinants of the place fields of hippocampal neurons. Nature 381:425-28. [aJPA, AT]

O’Keefe, J. \& Nadel, L. (1978) The hippocampus as a cognitive map. Oxford University Press. [aJPA, JB, DJM, IQW]

Okuno, H. \& Miyashita, Y. (1996) Expression of the transcription factor Zif268 in the temporal cortex of monkeys during visual paired associate learning. European Journal of Neuroscience 8:2118-28. [aJPA]

Olton, D. S., Becker, J. T. \& Handelmann, G. E. (1979) Hippocampus, space and memory. Behavioral and Brain Sciences 2:313-65. [JKF]

Olton, D. S., Walker, J. A. \& Woolf, W. A. (1982) A disconnection analysis of hippocampal function. Brain Research 233:241-53. [arJPA]

Owen, M. J. \& Butler, S. R. (1984) Does amnesia after transection of the fornix in monkeys reflect abnormal sensitivity to proactive interference? Behavioural Brain Research 14:183-92. [rJPA]

Otto, T. \& Eichenbaum, H. (1992a) Complementary roles of the orbital prefrontal cortex and the perirhinal-entorhinal cortices in an odor-guided delayednonmatching to sample task. Behavioral Neuroscience 106:762-75. [MJE]

(1992b) Neuronal activity in the hippocampus during delayed non-match to sample performance in rats: Evidence for hippocampal processing in recognition memory. Hippocampus 2:323-34. [aJPA]

Packard, M. G., Cahill, L. \& McGaugh, J. L. (1994) Amygdala modulation of hippocampal-dependent and caudate nucleus-dependent memory processes. Proceedings of the National Academy of Sciences USA 91:8477-81. [rJPA]

Paller, K. A., Acharya, A., Richardson, B. C., Plaisant, O., Shimamura, A. P., Reed B. R. \& Jagust, W. J. (1997) Functional neuroimaging of cortical dysfunction in alcoholic Korsakoff's syndrome. Journal of Cognitive Neuroscience 9:27793. [arJPA]

Papez, J. W. (1937) A proposed mechanism of emotion. Archives of Neurology and Psychology 38:725-43. [RDB, HJM]

Parker, A., Eacott, M. J. \& Gaffan, D. (1997) The recognition memory deficit caused by mediodorsal thalamic lesion in nonhuman primates: A comparison with rhinal cortex lesion. European Journal of Neuroscience 9:2423-31. [arJPA, JCD-A, AP]

Parker, A. \& Gaffan, D. (1997a) Mamillary body lesions in monkeys impair objectin-place memory: Functional unity of the fornix-mamillary system. Journal of Cognitive Neuroscience 9:512-21. [aJPA, AP]

(1997b) The effects of anterior thalamic and cingulate cortex lesions on "objectin -place” memory in monkeys. Neuropsychologia 35:1093-102. [aJPA, AP]

(1997c) Frontal/temporal disconnection in monkeys: Memory for strategies and memory for visual objects. Society for Neuroscience Abstracts 23:11. [AP]

(1998a) Interaction of frontal and perirhinal cortices in visual object recognition memory in monkeys. European Journal of Neuroscience 10:3044-57. [rJPA, AP]

(1998b) Memory after frontal/temporal disconnection in monkeys: Conditional and non-conditional tasks, unilateral and bilateral frontal lesions. Neuropsychologia 36:259-71. [AP]

(1998c) Memory systems in primates: Episodic, semantic and perceptual learning. In: Comparative neuropsychology, ed. D. Milner. Oxford University Press. [MJE]

(in press) Magnocellular mediodorsal thalamic ablation does not disrupt preoperatively learned visual object associations, but impairs both new acquisition of object associations and scene learning. Behavioral Neuroscience. [AP]

Parker, A., Wilding, E. \& Akerman, C. (1998) Novelty and memory encoding: Von Restorff effects in normal human and non-human primate recognition memory, and the role of frontal/perirhinal interaction. Journal of Cognitive Neuroscience 10:691-703. [AP]

Parkin, A. J. (1984) Memory and amnesia. Blackwell. [aJPA]

Parkin, A. J., ed. (1997) Case studies in the neuropsychology of memory. Psychology Press (Taylor \& Francis). [JKF]

Parkin, A. J., Dunn, J. C., Lee, C., O’Hara, P. F. \& Nussbaum, L. (1993) Neuropsychological sequelae of Wernicke's encephalopathy in a 20 -year- old woman: Selective impairment of a frontal memory system. Brain and Cognition 21:1-19. [aJPA]

Parkin, A. J. \& Hunkin, N. M. (1993) Impaired temporal context memory on anterograde but not retrograde tests in the absence of frontal pathology. Cortex 29:267-80. [aJPA]

Parkin, A. J. \& Leng, N. R. C. (1993) Neuropsychology of the amnesic syndrome. Erlbaum. [aJPA]

Parkin, A. J., Leng, N. R. C. \& Hunkin, N. M. (1990) Differential sensitivity to context in diencephalic and temporal lobe amnesia. Cortex 26:373-80. [aJPA]

Parkin, A. J., Yeomans, J. \& Bindschaedler, C. (1994) Further characterization of 
References/Aggleton \& Brown: Episodic memory, amnesia, and hippocampus

the executive memory impairment following frontal lobe lesions. Brain and Cognition 26:23-42. [aJPA]

Parkinson, J. A. \& Aggleton, J. P. (1994) The failure of context shifts to alter the recognition of faces: Implications for contextual deficits in amnesia. Cortex 30:351-54. [aJPA]

Parkinson, J. K., Murray, E. A. \& Mishkin, M. (1988) A selective mnemonic role for the hippocampus in monkeys: Memory for the location of objects. Journal of Neuroscience 8:4159-67. [aJPA, SMZ]

Pearce, J. M., Roberts, A. D. L. \& Good, M. (1998) Hippocampal lesions disrupt navigation based on cognitive maps but not heading vectors. Nature 396:7577. [rJPA]

Penfield, W. \& Mathieson, G. (1974) Memory: Autopsy findings and comments on the role of hippocampus in experiential recall. Archives of Neurology 31:14554. [aJPA]

Perfect, T. J., Mayes, A. R., Downes, J. J. \& Van Eijk, R. (1996) Does context discriminate recollection from familiarity in recognition memory? Quarterly Journal of Experimental Psychology 49A:797-813. [aJPA]

Pickering, A. D. (1997) New approaches to the study of amnesic patients: What can a neurofunctional philosophy and neural network methods offer? Memory 5:255-300. [ADP]

Porter, M. C. \& Mair, R. G. (1997) The effects of frontal cortical lesions on remembering depend on the procedural demand of tasks performed in the radial arm maze. Behavioural Brain Research 87:115-25. [RGM]

Poucet, B. (1993) Spatial cognitive maps in animals: New hypotheses on their structure and neural mechanisms. Psychological Review 100:163-82. [DJM]

Prepscius, C. \& Levy, W. B. (1994) Sequence prediction and cognitive mapping by a biologically plausible neural network. Proceedings of the INNS World Congress on Neural Networks 5:164-69. [ADP]

Rajaram, S. (1993) Remembering and knowing: Two means of access to the personal past. Memory and Cognition 21:89-102. [aJPA, BJK]

Ramus, S. J., Zola-Morgan, S. \& Squire, L. R. (1994) Effects of lesions of perirhinal cortex or parahippocampal cortex on memory in monkeys. Society for Neuroscience Abstracts 20:1074. [aJPA, SMZ]

Ratcliff, R., Van Zandt, T. \& McKoon, G. (1995) Process dissociation, singleprocess theories, and recognition memory. Journal of Experimental Psychology: General 124:353-74. [aJPA]

Rawlins, J. N. P. (1985) Associations across time: The hippocampus as a temporary memory store. Behavioural and Brain Sciences 8:479-528. [JKF]

Reed, J. M. \& Squire, L. R. (1997) Impaired recognition memory in patients with lesions limited to the hippocampal formation. Behavioural Neuroscience 111:667-75. [aJPA, SMZ]

Rempel-Clower, N. L., Zola, S., Squire, L. R. \& Amaral, D. G. (1996) Three cases of enduring memory impairment after bilateral damage limited to the hippocampal formation. Journal of Neuroscience 16:5233-55. [aJPA, SMZ]

Remy, M. (1942) Contribution a l'etude de la maladie de Korsakow. Monatsschrift für Psychiatrie und Neurologie 106:128-44. [aJPA]

Rescorla, R. A. (1967) Pavlovian conditioning and its proper control procedures. Psychological Review 74:71-80. [DJM]

Riches, I. P., Wilson, F. A. W. \& Brown, M. W. (1991) The effects of visual stimulation and memory on neurons of the hippocampal formation and the neighboring parahippocampal gyrus and inferior temporal cortex of the primate. Journal of Neuroscience 11:1763-79. [aJPA]

Rigges, H. E. \& Boles, R. S. (1944) Wernicke's encephalopathy: Clinical and pathological studies of 42 cases. Quarterly Journal Studies of Alcohol 5:36170. [aJPA]

Ringo, J. L. (1996) Stimulus specific adaptation in inferior temporal and medial temporal cortex of the monkey. Behavioural Brain Research 76:191-97. [aJPA]

Robinson, J. K. \& Mair, R. G. (1992) MK-801 prevents brain lesions and delayed non-matching to sample deficits produced by pyrithiamineinduced encephalopathy in rats. Behavioural Neuroscience 106:623-33. [RGM]

Roediger, H. L. \& McDermott, K. B. (1994) The problem of differing false-alarm rates for the process dissociation procedure: Comment on Verfaellie and Treadwell (1993) Neuropsychology 8:284-88. [aJPA]

Rolls, E. T. (1996) A theory of hippocampal function in memory. Hippocampus 6:601-20. [JKF]

Rolls, E. T., Cahusac, P. M. B., Feigenbaum, J. D. \& Miyashita, Y. (1993) Responses of single neurons in the hippocampus of the macaque related to recognition memory. Experimental Brain Research 93:299-306. [aJPA, DLH, AT

Rolls, E. T. \& O’Mara, S. M. (1995) View-responsive neurons in the primate hippocampal complex. Hippocampus 5:409-24. [aJPA, AT]

Rolls, E. T., Treves, A., Robertson, R. G., Georges-Francois, P. \& Panzeri, S. (1998) Information about spatial view in an ensemble of primate hippocampal cells. Journal of Neurophysiology 79:1797-813. [AT]

Romanes, G. J. (1889) Mental evolution in man. D. Appleton \& Co. [DJM]

Rombouts, S. A. R. B., Machielson, W. C. M., Witter, M. P., Barkhof, F.,
Lindeboom, J. \& Scheltens, P. (1997) Visual association encoding shows activation in the medial temporal lobe. A functional magnetic resonance imaging study. Hippocampus 7:594-601. [MPW]

Room, P. \& Groenewegen, H. J. (1986) Connections of the parahippocampal cortex in the cat. II. Subcortical afferents. Journal of Comparative Neurology 251:451-73. [MPW]

Rothblat, L. A. \& Kromer, L. F. (1991) Object recognition memory in the rat: The role of the hippocampus. Behavioural Brain Research 42:25-32. [aJPA]

Rugg, M. D., Fletcher, P. C., Frith, C. D., Frackowiak, R. S. J. \& Dolan, R. J. (1996) Differential activation of the prefrontal cortex in successful and unsuccessful memory retrieval. Brain 119:2073-83. [aJPA]

Rupniak, N. M. J. \& Gaffan, D. (1987) Monkey hippocampus and learning about spatially directed movements. Journal of Neuroscience 7:2331-37. [rJPA]

Russchen, F. T., Amaral, D. G. \& Price, J. L. (1987) The afferent input to the magnocellular division of the mediodorsal thalamic nucleus in the monkey Macaca fascicularis. Journal of Comparative Neurology 256:175-210. [arJPA, MPW]

Sakai, K. \& Miyashita, Y. (1991) Neuronal organisation for the long-term memory of paired associates. Nature 354:152-55. [MJE]

Saunders, R. C. \& Rosene, D. L. (1988) A comparison of the efferents of the amygdala and the hippocampal formation in the rhesus monkey: I. Convergence in the entorhinal, prorhinal, and perirhinal cortices. Journal of Comparative Neurology 271:153-84. [aJPA]

Saunders, R. C. \& Weiskrantz, L. (1989) The effects of fornix transection and combined fornix transection, mammillary body lesions and hippocampal ablations on object-pair association memory in the rhesus monkey. Behavioural Brain Research 35:85-94. [aJPA]

Savage, L. M., Sweet, A. J., Castillo, R. \& Langlais, P. J. (1997) The effects of lesions to thalamic lateral internal medullary lamina and posterior nuclei on learning, memory and habituation in the rat. Behavioural Brain Research 82:133-47. [aJPA]

Schacter, D. L. (1987) Memory, amnesia, and frontal lobe dysfunction. Psychobiology 15:21-36. [aJPA]

Schacter, D. L., Chiu, C.-Y, P. \& Ochsner, K. N. (1993) Implicit memory: A selective review. Annual Review of Neuroscience 16:159-82. [aJPA]

Schacter, D. L., Tharan, M., Cooprt, L. A. \& Rubens, A. B. (1991) Preserved priming of novel objects in patients with memory disorders. Journal of Cognitive Neuroscience 3:117-30. [aJPA]

Schacter, D. L., Verfaellie, M. \& Anes, M. D. (1997) Illusory memories in amnesic patients: Conceptual and perceptual false recognition. Neuropsychology 11:331-42. [APY]

Schacter, D. L., Verfaellie, M. \& Pradere, D. (1996) Neuropsychology of memory illusions: False recall and recognition in amnesic patients. Journal of Memory and Language 35(2):319-34. [APY]

Schnider, A., Gutbrod, K., Hess, C. W. \& Schroth, G. (1996) Memory without context: Amnesia with confabulation after infarction of the right capsular genu. Journal of Neurology, Neurosurgery, and Psychiatry 61:186-93. [aJPA]

Shaw, C. \& Aggleton, J. P. (1993) The effects of fornix and medial prefrontal lesions on delayed non-matching-to-sample by rats. Behavioural Brain Research 54:91-102. [aJPA]

(1995) Evidence for the independence of recognition and recency memory in amnesic subjects. Cortex 31:57-71. [aJPA]

Shibata, H. (1992) Topographic organization of subcortical projections to the anterior thalamic nuclei in the rat. Journal of Comparative Neurology 32: $3: 117-27$

(1993a) Direct projections from the anterior thalamic nuclei to the retrohippocampal region in the rat. Journal of Comparative Neurology 337:431-45. [aJPA, JCD-A]

(1993b) Efferent projections from the anterior thalamic nuclei to the cingulate cortex in the rat. Journal of Comparative Neurology 330:533-42.

(1996) Direct projections from the entorhinal area to the anteroventral and laterodorsal thalamic nuclei in the rat. Neuroscience Research 26:83-87. [aJPA]

Shimamura, A. P. (1995) Memory and frontal lobe function. In: The cognitive neurosciences, ed. M. S. Gazzaniga. MIT Press. [BJK]

Shimamura, A. P., Janowsky, J. S. \& Squire, L. R. (1990) Memory for the temporal order of events in patients with frontal lobe lesions and amnesic patients. Neuropsychologia 28:803-13. [aJPA]

Shuren, J. E., Jacobs, D. H. \& Heilman, K. M. (1997) Diencephallic temporal order amnesia. Journal of Neurological and Neurosurgical Psychiatry 62:16368. [MPW]

Sobotka, S. \& Ringo, J. L. (1993) Investigations of long-term recognition and association memory in unit responses from inferotemporal cortex. Experimental Brain Research 96:28-38. [aJPA]

Sobotka, S. \& Ringo, J. L. (1994) Stimulus specific adaptation in excited but not in inhibited cells in inferotemporal cortex of macaque. Brain Research 646:9599. [aJPA] 
Snowden, J. S., Goulding, P. J. \& Neary, D. (1989) Semantic dementia: A form of circumscribed cerebral atrophy. Behavioural Neurology 2:167-82. [KSG]

Snowden, J. S., Griffiths, H. L. \& Neary, D. (1996) Semantic-episodic memory interactions in semantic dementia: Implications for retrograde memory function. Cognitive Neuropsychology 13:1101-37. [KSG]

Squire, L. R. (1981) Two forms of human amnesia: An analysis of forgetting. Journal of Neuroscience 1:635-40. [aJPA]

(1992) Memory and the hippocampus: A synthesis from findings with rats, monkeys, and humans. Psychological Review 99:195-231. [JKF, ADP, SMZ]

Squire, L. R. \& Alvarez, P. (1995) Retrograde amnesia and memory consolidation: A neurobiological perspective. Current Opinion in Neurobiology 5:169-77. [aJPA, LN]

Squire, L. R., Amaral, D. G., Zola-Morgan, S., Kritchevsky, M. \& Press, G. (1989) Description of brain injury in the amnesic patient N.A. based on magnetic resonance imaging. Experimental Neurology 105:23-35. [aJPA]

Squire, L. R. \& Knowlton, B. J. (1995) Memory, hippocampus, and brain systems. In: The cognitive neurosciences, ed. M. Gazzinaga. MIT Press. [aJPA]

Squire, L. R., Knowlton, B. J. \& Musen, G. (1993) The structure and organization of memory. Annual Reviews in Psychology 44:453-95. [RDB]

Squire, L. R. \& Shimamura, A. P. (1986) Characterizing amnesic patients for neurobehavioural study. Behavioural Neuroscience 100:866-77. [aJPA]

Squire, L. R. \& Zola, S. (1996) Ischemic brain damage and memory impairment: A commentary. Hippocampus 6:546-52. [SMZ]

Squire, L. R. \& Zola-Morgan, S. (1991) The medial temporal lobe memory system. Science 253:1380-86. [aJPA, SMZ]

(1996) Ischemic brain damage and memory impairment: A commentary. Hippocampus 6:546-52. [aJPA]

Squire, L. R., Zola-Morgan, S. \& Chen, K. S. (1988) Human amnesia and animal models of amnesia: Performance of amnesic patients on tests designed for the monkey. Behavioural Neuroscience 102:210-21. [aJPA]

Steckler, T., Drinkenburg, W. H. I. M., Sahgal, A. \& Aggleton, J. P. (1998a) Recognition memory in rats - I. Concepts and classification.. Progress in Neurobiology 54:289-311. [RPK]

(1998b) Recognition memory in rats - II. Neuroanatomical substrates. Progress in Neurobiology 54:313-32. [ JCD-A]

Steele, K. \& Rawlins, J. N. P. (1993) The effects of hippocampectomy on performance by rats of a running recognition task using long lists of nonspatial items. Behavioural Brain Research 54:1-10. [aJPA]

Stern, C. E., Corkin, S., Gonzalez, G., Guimaraes, A. R., Baker, J. R., Jennings, P. J., Carr, C. A., Sugiura, R. M., Vedantham, V. \& Rosen, B. R. (1996) The hippocampal formation participates in novel picture encoding: Evidence from functional magnetic resonance imaging. Proceedings of the National Academy of Sciences, USA 93:8660-65. [LN]

Stuss, D. T., Eskes, G. A. \& Foster, J. K. (1994) Experimental neuropsychological studies of frontal lobe functions. In: Handbook of neuropsychology, vol. 9, ed. H. Spinnler \& F. Boller. Elsevier. [JKF]

Stuss, D. T., Guberman, A., Nelson, R. \& Larochelle, S. (1988) The neuropsychology of paramedian thalamic infarction. Brain and Cognition 8:348-78. [aJPA]

Sugita, K., Doi T., Mutsuga, N. \& Takaoka, Y. (1971) Clinical study of fornicotomy for psychomotor epilepsy and behavior disorder. In: Special topics in stereotaxis: Epilepsy, disorders of behaviour, and autonomic balance, ed. W. Umbach. Hippokrates. [aJPA]

Sutherland, R. J. \& Hoesing, J. M. (1993) Posterior cingulate cortex and spatial memory: A microlimnology analysis. In: Neurobiology of cingulate cortex and limbic thalamus, ed. B. A. Vogt \& M. Gabriel. Birkhauser. [aJPA]

Sutherland, R. J. \& Rodriguez, A. J. (1989) The role of the fornix/fimbria and some related subcortical structures in place learning and memory. Behavioural Brain Research 32:265-77. [aJPA]

Sutherland, R. J. \& Rudy, J. W. (1989) Configural association theory: The role of the hippocampal formation in learning, memory, and amnesia. Psychobiology 17:129-44. [SMZ]

Sutherland, R. J., Whishaw, I. Q. \& Kolb, B. (1988) Contributions of cingulate cortex to two forms of spatial learning and memory. Journal of Neuroscience 8:1863-72. [aJPA]

Suzuki, W. A. (1996a) The anatomy, physiology and functions of the perirhinal cortex. Current Opinion in Neurobiology 6:179-86. [aJPA, RGM]

(1996b) Neuroanatomy of the monkey entorhinal, perirhinal and parahippocampal cortices: Organization of cortical inputs and interconnections with amygdala and striatum. Seminars in the Neurosciences $8: 3-12 . \quad$ [aJPA]

Suzuki, W. A. \& Amaral, D. G. (1990) Cortical inputs to the CAl field of the monkey hippocampus originate from the perirhinal and parahippocampal cortex but not from area TE. Neuroscience Letters 115:43-48. [aJPA, RDB] (1994a) Perirhinal and parahippocampal cortices of the Macaque monkey: Cortical afferents. Journal of Comparative Neurology 350:497-533. [aJPA, RDB, SMZ

(1994b) Topographic organization of the reciprocal connections between the monkey entorhinal cortex and the perirhinal and parahippocampal cortices. Journal of Neuroscience 14:1856-77. [aJPA, RDB]

Suzuki, W. A., Miller, E. K. \& Desimone, R. (1995) Object and place memory in the monkey entorhinal cortex. Society for Neuroscience Abstracts 21:19. [aJPA]

Suzuki, W. A., Zola-Morgan, S., Squire, L. R. \& Amaral, D. G. (1993) Lesions of the perirhinal and parahippocampal cortices in the monkey produce longlasting memory impairment in the visual and tactual modalities. Journal of Neuroscience 13:2430-51. [aJPA]

Sweet, W. H., Talland, G. A. \& Ervin, F. R. (1959) Loss of recent memory following section of fornix. Transactions of the American Neurological Association 84:76-82. [aJPA]

Taube, J. S. (1995) Head direction cells recorded in the anterior thalamic nuclei of freely moving rats. Journal of Neuroscience 15:70-86. [arJPA]

Teng, E., Squire, L. R. \& Zola, S. (1997) Different memory roles for the parahippocampal and perirhinal cortices in spatial reversal. Society for Neuroscience Abstracts 23:12. [SMZ]

(1998) Effects of removal from the testing apparatus on delayed nonmatching to sample performance in monkeys with lesions of the hippocampal region. Society for Neuroscience Abstracts 24:17. [SMZ]

Thomas, G. J. \& Brito, G. N. O. (1980) Recovery of delayed alternation in rats after lesions in medial prefrontal cortex and septum. Journal of Comparative and Physiological Psychology 94:808-18. [aJPA]

Thomas, G. J. \& Spafford, P. S. (1984) Deficits for representational memory induced by septal and cortical lesions (singly and combined) in rats. Behavioural Neuroscience 98:394-404. [aJPA]

Thompson, R. F. (1990) Neural mechanisms of classical conditioning in mammals. Philosophical Transactions of the Royal Society London B 329:161-70. [aJPA]

Torvik, A. (1987) Topographic distribution and severity of brain lesions in Wernicke's encephalopathy. Clinical Neuropathology 6:25-29. [aJPA]

Treves, A., Georges-Francois, P., Panzeri, S., Robertson, R. G. \& Rolls, E. T. (1998) The metric content of spatial views as represented in the primate hippocampus. In: Neuronal circuits and networks, ed. V. Torre \& J. Nicholls.NATO ASI series, vol. 167. Springer. [AT]

Treves, A., Panzeri, S., Robertson, R. G., Georges-Francois, P. \& Rolls, E. T. (1996a) The emergence of structure in neuronal representations. Society for Neuroscience Abstracts 22:281. [AT]

Treves, A., Skaggs, W. E. \& Barnes, C. A. (1996b) How much of the hippocampus can be explained by functional constraints? Hippocampus 6:666-74. [AT]

Tucker, D. M., Roeltgen, D. P., Tully, R., Hartmann, J. \& Boxell, C. (1988) Memory dysfunction following unilateral transection of the fornix: A hippocampal disconnection syndrome. Cortex 24:465-72. [aJPA]

Tulving, E. (1983) Elements of episodic memory. Clarendon Press. [aJPA]

(1985a) Memory and consciousness. Canadian Psychologist 26(1):1-12. [aJPA, BJK, APY]

(1985b) How many memory systems are there? American Psychologist 40:38598. [SMZ]

(1991) Concepts in human memory. In: Memory: Organization and locus of change, ed. L. R. Squire, N. M. Weinberger, G. Lynch \& J. McGaugh. Oxford University Press. [SMZ]

Tulving, E., Kapur, S., Markowitsch, H. J., Craik, F. I. M., Habib, R. \& Houle, S. (1994) Neuroanatomical correlates of retrieval in episodic memory: Auditory sentence recognition. Proceedings of the National Academy of Sciences, USA 91:2012-15. [LN]

Tulving, E. \& Markowitsch, H. J. (1994) What do animal models of memory model? Behavioural and Brain Sciences 17:498-99. [aJPA]

(1998) Episodic and declarative memory: Role of the hippocampus. Hippocampus 8:198-204. [HJM]

Tulving, E., Markowitsch, H. J., Craik, F. I. M., Habib, R. \& Houle, S. (1996) Novelty and familiarity activations in PET studies and memory encoding retrieval. Cerebral Cortex 6:71-79. [aJPA]

Ungerleider, L. G. \& Mishkin, M. (1982) Two cortical visual systems. In: Analysis of visual behavior, ed. D. J. Ingle, M. A. Goodale \& R. J. W. Mansfield. MIT Press. [EEK]

Valenstein, E., Bowers, D., Verfaellie, M., Heilman, K. M., Day, A. \& Watson, R. T. (1987) Retrosplenial amnesia. Brain 110:1631-46. [aJPA]

Vandenberghe, R., Dupont, P., Bormans, G., Mortelmans, L. \& Orban, G. (1995) Rapid communication blood flow in human anterior temporal cortex decreases with stimulus familiarity. Neuroimage 2:306-13. [aJPA]

Van der Werf, Y. D., Weerts, J. G. E., Jolles, J., Witter, M. P., Lindeboom, J. \& Scheltens, P. (1998) Neuropsychological correlates of a right unilateral lacunar thalamic infarction. Journal of Neurological and Neurosurgical Psychiatry (in press). [MPW]

Van Hoesen, G. W. (1997) Ventromedial temporal lobe anatomy, with comments on Alzheimer's disease and temporal injury. Journal of Neuropsychiatry and Clinical Neurosciences 9:331-41. [KSG]

Vargha-Khadem, F., Gadian, D. G., Watkins, K. E., Connelly, A., Van Paesschen, 
References/Aggleton \& Brown: Episodic memory, amnesia, and hippocampus

W. \& Mishkin, M. (1997) Differential effects of early hippocampal pathology on episodic and semantic memory. Science 277:376-80. [arJPA, KSG, SMZ]

Verfaellie, M. (1994) A re-examination of recognition memory in amnesia: Reply to Roediger and McDermott. Neuropsychology 8:289-92. [aJPA]

Verfaellie, M. \& Treadwell, J. R. (1993) Status of recognition memory in amnesia. Neuropsychology 7(1):5-13. [arJPA, APY]

Victor, M., Adams, R. D. \& Collins, G. H. (1971) The Wernicke-Korsakoff syndrome. Blackwell. [aJPA]

(1989) The Wernicke-Korsakoff syndrome and related neurologic disorders due to alcoholism and malnutrition, 2nd edition. F. A. Davis. [aJPA, RGM]

Victor, M. \& Agamanolis, D. (1990) Amnesia due to lesions confined to the hippocampus: A clinical-pathologic study. Journal of Cognitive Neuroscience 2:246-57. [aJPA]

Vogt, B. A., Gabriel, M. Vogt, L. J., Poremba, A., Jensen, E. L., Kubota, Y. \& Kang, E. (1991) Muscarinic receptor binding increases in anterior thalamus and cingulate cortex during discriminative avoidance learning. Journal of Neuroscience 11:1508-14. [aJPA]

Volpe, B. T., Holtzman, J. D. \& Hist, W. (1986) Further characterization of patients with amnesia after cardiac arrest: Preserved recognition memory. Neurology 36:408-11. [aJPA]

Von Cramon, D. Y. (1992) Focal cerebral lesions damaging (subcortical) fibre projections relating to memory and learning functions in man. In: Neuropsychological disorders associated with subcortical lesions, ed. G. Valler, S. F. Cappa \& C. W. Wallesch. Oxford University Press. [aJPA]

Von Cramon, D. Y., Hebel, N. \& Schuri, U. (1985) A contribution to the anatomical basis of thalamic amnesia. Brain 108:993-1008. [RGM, MPW]

Von Cramon, D. Y., Markowitsch, H. J. \& Schuri, U. (1993) The possible contributions of the septal region to memory. Neuropsychologia 31:1159-80. $[\mathrm{HJM}$

Wagner, A. D., Stebbins, G. T., Masciari, F., Fleischman, D. A. \& Gabriel, J. D. E. (1998) Neuropsychological dissociation between recognition familiarity and perceptual priming in visual long-term memory. Cortex 34:493-511. [rJPA]

Wan, H., Aggleton, J. P. \& Brown, M. W. (1999) Different contributions of the hippocampus and perirhinal cortex to recognition memory. Journal of Neuroscience 19:1142-48. [aJPA, JB]

Warburton, E. C. \& Aggleton, J. P. (1999) Differential effects in the Morris water maze following cytotoxic lesions of the anterior thalamus and fornix transection. Behavioural Brain Research 98:27-38. [arJPA]

Warburton, E. C., Aggleton, J. P. \& Muir, J. L. (1998) Comparing the effects of selective cingulate cortex and cingulum bundle lesions on a spatial navigation task. European Journal of Neuroscience 10:622-34. [arJPA]

Warburton, E .C., Baird, A. L. \& Aggleton, J. P. (1997) Assessing the magnitude of the allocentric spatial deficit associated with complete loss of the anterior thalamic nuclei in rats. Behavioural Brain Research 87:223-32. [aJPA, JCD-A]

Warrington, E. K. (1975) Selective impairment of semantic memory. Quarterly Journal of Experimental Psychology 27:635-57. [KSG] (1984) The recognition memory test. NFER-Nelson. [ar]PA, KSG, MPW]

Warrington, E. K. \& Weiskrantz, L. (1974) The effect of prior learning on subsequent retention in amnesia patients. Neuropsychologia 12:419-28. [aJPA]

Weiskrantz, L. (1985) On issues and theories of the human amnesic syndrome. In: Memory systems of the brain, ed. N. M Weinberger, J. L. McGaugh \& G. Lynch. Guilford Press. [aJPA]

(1990) Problems of learning and memory: One or multiple memory systems? Philosophical Transactions of the Royal Society London B. 329:99-108. [aJPA]

Wheeler, M. A., Stuss, D. T. \& Tulving, E. (1995) Frontal lobe damage produces episodic memory impairment. Journal of the International Neuropsychological Society 1:525-36. [aJPA]

Whishaw, I. Q. (1998) Place learning in hippocampal rats and the path integration hypothesis. Neuroscience and Biobehavioural Reviews 22:209-20. [IQW]

Whishaw, I. Q., Cassel, J.-C. \& Jarrard, L. E. (1995) Rats with fimbria-fornix lesions display a place response in a swimming pool: A dissociation between getting there and knowing where. Journal of Neuroscience 15:5779-88. [IQW]

Whishaw, I. Q. \& Jarrard, L. E. (1995) Similarities vs. differences in place learning and circadian activity in rats after fimbria-fornix section or ibotenate removal of hippocampal cells. Hippocampus 5:595-604. [IQW]

(1996) Evidence for extrahippocampal involvement in place learning and hippocampal involvement in path integration. Hippocampus 6:513-24. [AP, IQW]

Whishaw, I. Q. \& Maaswinkel, H. (1998) Rats with fimbria-fornix lesions are impaired in path integration: A role of the hippocampus in "sense of direction." Journal of Neuroscience 18:3050-58. [IQW]

Whishaw, I. Q., McKenna, J. E. \& Maaswinkel, H. (1997) Hippocampal lesions and path integration. Current Opinion in Neurobiology 7:228-34. [AP, IQW]

Whishaw, I. Q. \& Tomie, J. (1997a) Piloting and dead reckoning dissociated by fimbria-fornix lesions in a rat food carrying task. Behavioural Brain Research 89:87-97. [IQW]

(1997b) Perseveration on place reversals in spatial swimming pool tasks: Further evidence for place learning in hippocampal rats. Hippocampus 7:361-70. [IQW]

Wiig, K. A. \& Bilkey, D. K. (1994) The effects of perirhinal cortical lesions on spatial reference memory in the rat. Behavioural Brain Research 63:101-109. [DGM]

(1995) Lesions of rat perirhinal cortex exacerbate the memory deficit observed following damage to the fimbria-fornix. Behavioural Neuroscience 109:62030. [aJPA]

Wiig, K. A., Booth, S. J., Lui, P., Cooper, L. \& Bilkey, D. K. (1995) The effects of separate and combined lesions of perirhinal cortex, fornix and septum on memory in the rat. Society for Neuroscience Abstracts 2:1935. [MJE]

Wilding, E. L. \& Rugg, M. D. (1996) An event-related potential study of recognition memory with and without retrieval of source. Brain 119:889-905. [aJPA]

Wilson, B., Baddeley, A. \& Kapur, N. (1995) Dense amnesia in a professional musician following herpes simplex virus encephalitis. Journal of Clinical and Experimental Neuropsychology 17:668-81. [NK]

Witter, M. P. \& Amaral, D. G. (1991) Entorhinal cortex of the monkey: V Projections to the dentate gyrus, hippocampus, and subicular complex. Journal of Comparative Neurology 307:437-39. [aJPA]

Witter, M. P. \& Groenewegen, H. J. (1986) Connections of the parahippocampal cortex in the cat. III. Cortical and thalamic efferents. Journal of Comparative Neurology 252:1-31. [MPW]

Witter, M. P., Groenewegen, H. J., Lopes da Silva, F. H. \& Lohman, A. H. M (1989) Functional organization of the extrinsic and intrinsic circuitry of the parahippocampal region. Progress in Neurobiology 33:161-253. [aJPA]

Wood, E. R., Mumby, D. G., Pinel, J. P. J. \& Phillips, A. G. (1993) Impaired object recognition memory in rats following ischemia-induced damage to the hippocampus. Behavioural Neuroscience 107:51-62. [aJPA]

Wood, E. R. \& Phillips, A. G. (1991) Deficits on a one trial object recognition task by rats with hippocampal CAl lesions produced by cerebral ischemia. Neuroscience Research Communications 9:177-82. [aJPA]

Woods, B. T., Schoene, W. \& Kneisley, L. (1982) Are hippocampal lesions sufficient to cause lasting amnesia? Journal of Neurology, Neurosurgery and Psychiatry 45:243-47. [aJPA]

Woolsey, R. M. \& Nelson, J. S. (1975). Asymptomatic destruction of the fornix in man. Archives of Neurology 32:566-68. [aJPA]

Xiang, J.-Z. \& Brown, M. W. (1997) Neuronal encoding of the prior occurrence of visual stimuli in rhinal cortex and area TE of the monkey. Brain Research Association Abstracts 14:42. [aJPA]

(1998) Differential neuronal encoding of novelty, familiarity and recency in regions of the anterior temporal lobe. Neuropharmacology (in press). [arJPA]

Yoneda, Y., Mori, E., Yamashita, H. \& Yamadori, A. (1994) MRI volumetry of medial temporal lobe structures in amnesia following herpes simplex encephalitis. European Neurology 34:243-52. [rJPA, NK]

Yonelinas, A. P. (1994) Receiver-operating characteristics in recognition memory: Evidence for a dual process model. Journal of Experimental Psychology: Learning, Memory and Cognition 20:1341-54. [aJPA, ADP, APY]

(1997) Recognition memory ROCs for item and associative information: The contribution of recollection and familiarity. Memory and Cognition 25:74763. [aJPA]

Yonelinas, A. P., Dobbins, I., Szymanski, M. D., Dhaliwal, H. S. \& King, L. (1996) Signal-detection, threshold, and dual-process models of recognition memory: ROCs and conscious recollection. Consciousness and Cognition 5:418-41. [ADP]

Yonelinas, A. P. \& Jacoby, L. L. (1995) The relation between remembering and knowing as bases for recognition: Effects of size congruency. Journal of Memory and Language 34:622-43. [aJPA]

Yonelinas, A. P., Kroll, N. E. A., Dobbins, I. G., Lazzara, M. \& Knight, R. T. (1998) Recollection and familiarity deficits in amnesia: Convergence of rememberknow, process dissociation, and receiver operating characteristic data. Neuropsychology 12:323-39. [APY]

Zhang, Y. P., Burk, J. A., Glode, B. M. \& Mair, R. G. (1998) The effects of thalamic and olfactory cortical lesions on continuous olfactory DNMTS and olfactory discrimination in the rat. Behavioural Neuroscience 112:39-53. [RGM]

Zhu, X. O., Brown, M. W. \& Aggleton, J. P. (1995a) Neuronal signalling of information important to visual recognition memory in rat rhinal and neighbouring cortices. European Journal of Neuroscience 7:753-65. [arjPA]

Zhu, X. O., Brown, M. W., McCabe, B. J. \& Aggleton, J. P. (1995b) Effects of the novelty or familiarity of visual stimuli on the expressions of the immediate early gene c-fos in rat brain. Neuroscience 69:821-29. [arJPA]

Zhu, X. O., McCabe, B. J., Aggleton, J. P. \& Brown, M. W. (1996) Mapping recognition memory through the differential expression of the immediate 
References/Aggleton \& Brown: Episodic memory, amnesia, and hippocampus

early gene c-fos induced by novel or familiar visual stimulation. Neuroreport 7:1871-75. [aJPA, JB]

(1997) Differential activation of the hippocampus and perirhinal cortex by novel visual stimuli and a novel environment. Neuroscience Letters 229:141-43. [aJPA]

Zola, S. \& Squire, L. R. (in press) Memory and brain systems. In: The Oxford handbook of memory, ed. E. Tulving \& F. Craik. Oxford University Press. [SMZ]

Zola, S., Teng, E., Clark, R. E., Stefanacci, L., Buffalo, E. A. \& Squire, L. R. (1998) Impaired recognition memory and simple discrimination learning in monkeys following lesions limited to the hippocampal region made by radio frequency., ischemia, or ibotenic acid. Society for Neuroscience Abstracts 24:17. [SMZ]

Zola-Morgan, S., Dabrowska, J., Moss, M. \& Mahut, H. (1983) Enhanced preference for perceptual novelty in the monkey after section of the fornix but not after ablation of the hippocampus. Neuropsychologia 21:433-54. [aJPA]

Zola-Morgan, S. \& Squire, L. R. (1985a) Amnesia in monkeys after lesions of the mediodorsal nucleus of the thalamus. Annals of Neurology 17:558-64. [arJPA]

(1985b) Medial temporal lesions in monkeys impair memory on a variety of tasks sensitive to human amnesia. Behavioural Neuroscience 99:22-34. [aJPA, DGM]

(1986) Memory impairment in monkeys following lesions limited to the hippocampus. Behavioural Neuroscience 100:155-60. [aJPA]

(1993) Neuroanatomy of memory. Annual Review of Neurocience 16:547-63. [aJPA]
Zola-Morgan, S., Squire, L. R. \& Amaral, D. G. (1986) Human amnesia and the medial temporal region: Enduring memory impairment following bilateral lesions limited to field CA1 of the hippocampus. Journal of Neuroscience 10:2950-67. [aJPA, NK, SMZ]

(1989a) Lesions of the hippocampal formation but not lesions of the fornix or the mammillary nuclei produce long-lasting memory impairment in monkeys. Journal of Neuroscience 9:898-913. [aJPA]

Zola-Morgan, S., Squire, L. R., Amaral, D. G. \& Suzuki, W. A. (1989b) Lesions of perirhinal and parahippocampal cortex that spare the amygdala and hippocampal formation produce severe memory impairment. Journal of Neuroscience 9:4355-70. [aJPA]

Zola-Morgan, S., Squire, L. R., Clower, R. P. \& Rempel, N. L. (1993) Damage to the perirhinal cortex exacerbates memory impairment following lesions to the hippocampal formation. Journal of Neuroscience 13:251-65. [aJPA, SMZ]

Zola-Morgan, S., Squire, L. R. \& Mishkin, M. (1982) The neuroanatomy of amnesia: Amygdala-hippocampal versus temporal stem. Science 218:1337-39. [aJPA]

Zola-Morgan, S., Squire, L. R. \& Ramus, S. J. (1994) Severity of memory impairment in monkeys as a function of locus and extent of damage within the medial temporal lobe memory system. Hippocampus 4:483-94. [SMZ]

Zola-Morgan, S., Squire, L. R., Rempel, N. L., Clower, R. P. \& Amaral, D. G. (1992) Enduring memory impairment in monkeys after ischemic damage to the hippocampus. Journal of Neuroscience 12:2582-96. [arJPA, SMZ] 